Portland State University

PDXScholar

Summer 8-6-2018

\title{
Thiophene-Based Molecular Sensors towards the Selective Detection of Mercury(II) and Other Metals
}

Austin Kazuo Shigemoto

Portland State University

Follow this and additional works at: https://pdxscholar.library.pdx.edu/open_access_etds

Part of the Chemistry Commons

Let us know how access to this document benefits you.

\section{Recommended Citation}

Shigemoto, Austin Kazuo, "Thiophene-Based Molecular Sensors towards the Selective Detection of Mercury(II) and Other Metals" (2018). Dissertations and Theses. Paper 4517.

https://doi.org/10.15760/etd.6401

This Dissertation is brought to you for free and open access. It has been accepted for inclusion in Dissertations and Theses by an authorized administrator of PDXScholar. Please contact us if we can make this document more accessible: pdxscholar@pdx.edu. 
Thiophene-Based Molecular Sensors towards the Selective Detection of Mercury(II) and Other Metals

by

Austin Kazuo Shigemoto

A dissertation submitted in partial fulfillment of the requirements for the degree of

\author{
Doctor of Philosophy \\ in \\ Chemistry
}

Dissertation Committee:

Theresa McCormick, Chair

Robert Strongin

Mark Woods

Raj Solanki

Portland State University

2018 


\begin{abstract}
This work describes several thiophene-based molecular sensors and various modifications aimed to improve and understand the photophysical and supramolecular properties, such as the association constant $\left(K_{\mathrm{a}}\right)$ and selectivity, towards the development of a selective mercury(II) sensor. From the first generation of sensors containing pyridine and thiophene groups, it was determined that thiophene can offer good selectivity for mercury(II) against other transition metal ions, and provide a ratiometric absorption and fluorescent response. The projects following this focused on improving the $K_{\mathrm{a}}$ of the first generation of sensors through several different strategies. Substitution of thiophene for dibenzothiophene was shown to improve the $K_{\mathrm{a}}$ however this resulted in less than ideal photophysics of the dibenzothiophene sensors with absorption and emission in the UVregion. In addition to the effect of the chelating group was examined by incorporating imidazole, and thiazole rings, to compare to the original pyridyl chelating group employed. From this it was determined that pyridine offered the greatest $K_{\mathrm{a}}$ and selectivity for mercury(II). Following this electron-donating groups, including alcohol, octaethyleneglycol monomethyl ether, and amine, were added to a sensor, 2,5-bis(2pyridyl)thiophene, as an alternative strategy to improving the $K_{\mathrm{a}}$. Initially these functional groups were placed on the pyridine ring which caused a great increase in affinity for transition metal ions such as iron(II) and copper(II), however this translated to a loss in selectivity. In the final project I functionalized the thiophene ring with the same electrondonating groups which resulted in an increased $K_{\mathrm{a}}$ and maintained good selectivity for the
\end{abstract}


mercury(II) ion, though iron(III) was still a competitive binder. In addition to this one of these thiophene functionalized sensors, 2,5-di(pyridin-2-yl)thiophene-3,4-diol, was shown to have a specific response to copper(II), iron(III), lead(II) and mercury(II) suggesting it could be used as a model for the development of a small-molecule multiplex sensor. Herein I will describe this work in greater detail and focus on the effects the modifications discussed had on the $K_{\mathrm{a}}$ and selectivity for the mercury(II) ion. 


\section{Dedication}

This work is dedicated to my parents, grandmother and all who have supported me throughout the years. 


\section{Acknowledgments}

I would like to thank several people for their continued support and assistance in completing this work. Dr. Mark Woods, Dr. Robert Strongin, and Dr. Raj Solanki for their advice and critiques helping me to complete this work. Dr. Theresa McCormick for your mentoring and patience, teaching me to go well beyond what I thought I was capable of accomplishing on my own. My Grandmother for her ongoing support and faith in me, which helped me through the hardest times. My mother and late father for their guidance and support. 


\section{Table of Contents}

$\begin{array}{ll}\text { Abstract } & \text { i }\end{array}$

Dedication iii

Acknowledgments $\quad$ iv

List of Tables $\quad$ x

List of Figures $\quad$ xii

List of Symbols and Abbreviations $\quad$ xx

Chapter 1. Introduction 1

1.1 Metal Ions of Interest 2

1.2 Designing Fluorescent and Colorimetric Sensors 5

1.2.1 The Association Constant and Strategies for the Design of Chemical Sensors 5

1.2.2 Ratiometric, Multiplex and Fluorescent Sensors 8

1.2.3 Characteristics to Consider in the Design of Fluorescent and Colorimeteric

$\begin{array}{ll}\text { Molecular Sensors } & 12\end{array}$

\subsection{Coordination Chemistry of Thiophene and Applications as a Molecular}

\section{Sensor 14}

\subsection{Summary of Work}


$\begin{array}{lll}2.1 & \text { Materials and Methods } & 20\end{array}$

$\begin{array}{lll}2.2 & \text { Synthesis of Sensors } & 21\end{array}$

2.2.1 Preparation of 2,5-bis(2-pyridyl)thiophene (L2) 21

2.2.2 Synthesis of 2,6-bis(2-thienyl)pyridine (L3) 21

2.2.3 Synthesis of 2-(dibenzo[b,d]thiophen-4-yl)pyridine (BT1) 22

2.2.4 Synthesis of 2-(dibenzo[b,d]thiophen-4-yl)-1H-imidazole (BT2) 23

2.2.5 Synthesis of 2-(dibenzo[b,d]thiophen-4-yl)thiazole (BT3)

2.2.6 Synthesis of 6,6'-thiophene-2,5-diyl)bis(pyridine-3-ol) (P1) 24

2.2.7 Synthesis of 2,5-bis(5-((2,5,8,11,14,17,20-heptaoxadocosan-22-

yl)oxy)pyridine-2-yl)thiophene (P2) 25

2.2.8 Synthesis of 6,6'-(thiophene-2,5-diyl)bis(pyridine-3-amine) (P3) 26

2.2.9 Synthesis of 2,2'-(3,4-dimethoxythiophene-2,5-diyl)dipyridine (T1) 27

2.2.10 Synthesis of 2,5-di(pyridin-2-yl)thiophene-3,4-diol (T2) 28

2.2.11 Synthesis of 2,2'-(3,4-bis((2,5,8,11,14,17,20-heptaoxadocosan-22-

yl)oxy)thiophene-2,5-diyl)dipyridine (T3) 28

2.2.12 Synthesis of 2,2'-(3,4-dinitrothiophene-2,5-diyl)dipyridine 30

2.2.13 Synthesis of 2,5-di(pyridin-2-yl)thiophene-3,4-diamine (T4) 30

$\begin{array}{lll}2.3 & \text { Measurement of Quantum Yields } & 31\end{array}$

2.4 General Procedure for Job's Plots 32

2.5 General Procedure for Absorption and Emission Titrations 32

2.6 General Procedure for ${ }^{1} \mathrm{H}$ NMR Experiments 32 
2.7 General Procedure for Competition Experiments

2.8 Computational Chemistry

Chapter 3. Thiophene-Based Ratiometric Sensors in Organic Solvent

3.1 Introduction

3.2 Results

3.2.1 Ligand Synthesis and Structure $\quad 35$

3.2.2 Photophysical Properties of Ligands 37

3.2.3 Photophysical Response to Mercury 39

3.2.4 NMR Titrations for Structure Determination 43

3.2.5 Selectivity towards Mercury(II) 46

$\begin{array}{lll}3.3 & \text { Conclusion } & 47\end{array}$

Chapter 4. Dibenzothiophene-Based Ligands as Chemical Sensors for Mercury(II)

49

4.1 Introduction $\quad 49$

$\begin{array}{lll}4.2 & \text { Results } & 50\end{array}$

$\begin{array}{lll}\text { 4.2.1 Synthesis and Structure of BT1-BT3 } & 50\end{array}$

4.2.2 Photophysics of Dibenzothiophene ligands 52

4.2.3 Photophysical Response to $\mathrm{Hg}^{2+} \quad 53$

4.2.4 ${ }^{1}$ H NMR Experiments for Structure Determination 57

4.2.5 Selectivity of BT1-BT3 for Mercury(II) 59

$\begin{array}{lll}4.3 & \text { Conclusion } & 60\end{array}$ 
Chapter 5. Evaluation of the Effects on Supramolecular Properties by

Functionalization of a Thiophene-Based Fluorescent Mercury(II) Sensor $\quad 62$

$\begin{array}{lll}5.1 & \text { Introduction } & 62\end{array}$

$\begin{array}{lll}5.2 & \text { Results } & 63\end{array}$

5.2.1 Synthesis and Structure of Pyridine-Functionalized Ligands 63

5.2.2 Photophysical Properties of Pyridine-Functionalized Ligands in $\mathrm{CH}_{3} \mathrm{CN} \quad 66$

5.2.3 Photophysical Properties of Pyridine-Functionalized Ligands in Mixed

$\begin{array}{ll}\text { Aqueous Media } & 68\end{array}$

5.2.4 Interaction of Pyridine-Functionalized Ligands with Mercury(II) in $\mathrm{CH}_{3} \mathrm{CN} 70$

5.2.5 Interaction of Pyridine-Functionalized ligands with Mercury(II) in Mixed

$\begin{array}{ll}\text { Aqueous Media } & 79\end{array}$

5.2.6 ${ }^{1} \mathrm{H}$ NMR Experiments for Structure Determination $\quad 81$

5.2.7 Selectivity towards Mercury(II) $\quad 84$

$\begin{array}{lll}5.3 & \text { Conclusions } & 86\end{array}$

Chapter 6. Effects of Functionalization of the Thiophene Ring on Supramolecular Properties and their Potential as Single-Molecule Multiplex Sensors $\quad 88$

$\begin{array}{llr}\text { 6.1 Introduction } & 88\end{array}$

$\begin{array}{llr}6.2 & \text { Results } & 89\end{array}$

6.2.1 Ligand Synthesis and Structure $\quad 89$

6.2.2 Photophysical Properties of Thiophene-Functionalized Sensors 92

6.2.3 Reactivity of $\mathbf{T 1}$ with $\mathrm{Cu}^{2+}, \mathrm{Fe}^{3+}, \mathrm{Pb}^{2+}$, and $\mathrm{Hg}^{2+}$

6.2.4 Reactivity of $\mathbf{T} 2$ to $\mathrm{Cu}^{2+}, \mathrm{Fe}^{3+}, \mathrm{Pb}^{2+}$ and $\mathrm{Hg}^{2+} \quad 102$ 
6.2.5 Reactivity of $\mathbf{T} 3$ to $\mathrm{Cu}^{2+}, \mathrm{Fe}^{3+}, \mathrm{Pb}^{2+}$ and $\mathrm{Hg}^{2+}$

6.2.6 Reactivity of $\mathbf{T} 4$ to $\mathrm{Cu}^{2+}, \mathrm{Fe}^{3+}, \mathrm{Pb}^{2+}$ and $\mathrm{Hg}^{2+}$

6.3 Conclusion

Chapter 7. Concluding Remarks

REFERENCES 


\section{List of Tables}

Table 1: Relative equilibrium constants of various $\eta^{1}$ sulfur-coordinated thiophene ligands compared to the nonfunctionalized thiophene, for the equilibrium described in Figure $5 .^{72}$ 17

Table 2: Photophysical properties of L1-L3 and the complexes formed in solution with

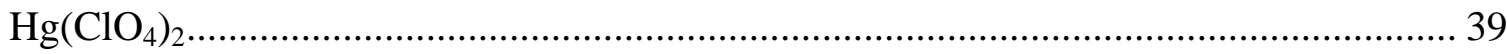

Table 3: Summary of photophysical data for BT1-BT3 obtained in $\mathrm{CH}_{3} \mathrm{CN}$ at $3 \times 10^{-5} \mathrm{M}$ concentration. Reported absorption wavelengths and molar absorptivities are those in the visible region 53

Table 4: Overall association constants obtained from absorption titration data in $\mathrm{CH}_{3} \mathrm{CN}$.

Table 5: Summary of photophysical data of 1-P3 acquired at $1 \times 10^{-5} \mathrm{M}$ in $\mathrm{CH}_{3} \mathrm{CN}$...... 67

Table 6: Association constant data obtained from fits of absorption titration data.

Stepwise data constants are reported along with the overall binding constant for compounds L1, P1 and P2.

Table 7: Summary of photophysical data of T1-T4 93

Table 8: Association constants for copper(II), iron(III), lead(II) and mercury(II) with T1 in $\mathrm{CH}_{3} \mathrm{CN}$ obtained from absorption titrations. 98

Table 9: Absorbance $\lambda_{\max }$ of the three products formed with T2 and either copper(II), iron(III), lead(II) or mercury(II) in $\mathrm{CH}_{3} \mathrm{CN}$ at $1 \times 10^{-5} \mathrm{M}$. 103 
Table 10: Association constant data for the binding of T2 to copper(II), iron(III), and mercury(II) in $\mathrm{CH}_{3} \mathrm{CN}$ obtained from absorption titrations..................................... 112

Table 11: Association constants for T3 with copper(II), iron(III), lead(II), and mercury(II) in $\mathrm{CH}_{3} \mathrm{CN}$ obtained from absorption titrations.

Table 12: Association constants for T4 with copper(II), iron(III), lead(II) and mercury(II) in $\mathrm{CH}_{3} \mathrm{CN}$ obtained from absorption titrations. 


\section{List of Figures}

Figure 1: Visual represntation of (a) 'turn-on' sensor, (b) 'turn-off' sensor, and (c) ratiometric sensor. Images obtained from sensors that are discussed later in this

document

Figure 2: Visual representation of a colorimetric sensor changing color in response to a metal analyte. 13

Figure 3: Structural depiction of BODIPY (left) and rhodamine (right) dyes. 14 Figure 4: Structural representation of previous molecular sensors incorporating a thienyl moiety. $^{32,46,48,51}$....... 15

Figure 5: Structural depiction of the possible coordination modes from left to right:

$\eta^{5}$ coordination via all 5 atoms, $\eta^{1}$ carbon-coordination, and $\eta^{1}$ sulfur-coordination. ${ }^{54} . .16$ Figure 6: Displacement equilibrium expression of an $\eta^{1}$ sulfur-coordinated thiophene by a modified thiophene ligand, identified by $\mathrm{Th}^{*}{ }^{72}$ 17

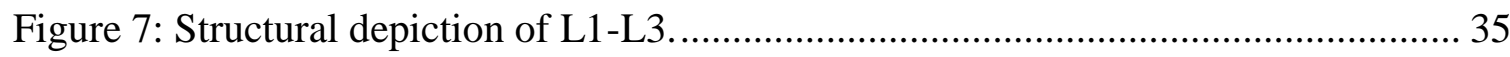

Figure 8: Optimized structures of (a) L1 (b) L2 and (c) L3 (B3LYP /6-311 + G(d)) ..... 37 Figure 9: (a) Absorption spectra of L1 (solid), L2 (dots), and L3 (dashes) (b) Emission spectra of L1 $\left(\lambda_{\mathrm{ex}}=297 \mathrm{~nm}\right.$, solid $), \mathrm{L} 2\left(\lambda_{\mathrm{ex}}=340 \mathrm{~nm}\right.$, dots $)$ and L3 $\left(\lambda_{\mathrm{ex}}=297 \mathrm{~nm}\right.$, dashes) 38

Figure 10: Change in (a) absorption of L1 (isobestic point $=314 \mathrm{~nm}$ ), (c) absorption of L2 to 20.0 eq, (e) absorption of L2 to 300 eq, (g) absorption of L3 (isobestic point = 344 $\mathrm{nm})$, (b) emission of L1 $\left(\lambda_{\mathrm{ex}}=297 \mathrm{~nm}\right)$, (d) emission of L2 $\left(\lambda_{\mathrm{ex}}=340 \mathrm{~nm}\right)$ to $20.0 \mathrm{eq}$, (f) 
emission of L2 $\left(\lambda_{\mathrm{ex}}=340 \mathrm{~nm}\right)$ to $250 \mathrm{eq}$, (h) emission of L3 $\left(\lambda_{\mathrm{ex}}=297 \mathrm{~nm}\right)$ with

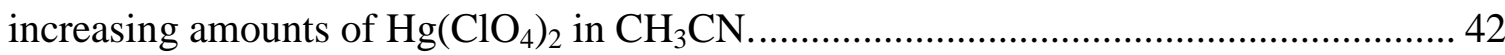

Figure 11: Change in ${ }^{1} \mathrm{H}$ NMR spectrum with increasing amounts of $\mathrm{Hg}\left(\mathrm{ClO}_{4}\right)_{2}$ for (a) $\mathrm{L} 1$, (b) L2, and (c) L3 in $\mathrm{CD}_{3} \mathrm{CN}$. Proposed structures with labeled protons are overlaid for each spectrum.

Figure 12: Emission of (a) L1, (b) L2, and (c) L3 at 410, 435, and 457 nm, respectively, before addition of metal (white bars), after the addition of 5 eq. of metal (grey bars), and after addition of 5 eq. of $\mathrm{Hg}\left(\mathrm{ClO}_{4}\right)_{2}$ in the presence of 5 eq. of metal salt (black bars)... 47 Figure 13: Structural depiction of BT1-BT3.

Figure 14: Absorption (a) and emission (b) spectrum of BT1-BT3 in $\mathrm{CH}_{3} \mathrm{CN}$ at $3 \times 10^{-}$ ${ }^{5} \mathrm{M}$

Figure 15: Absorption (a-b \& e-f) and emission (c-d \& g-h) titrations of BT1 and BT2 respectively with increasing concentrations of mercury(II) in $\mathrm{CH}_{3} \mathrm{CN}$ at $3 \times 10^{-5} \mathrm{M}$. Emission spectra were obtained by excitation at $345 \& 330 \mathrm{~nm}$ respectively

Figure 16: Change in (a) absorption and (b) emission of BT3 in response to increasing amounts of mercury(II) in $\mathrm{CH}_{3} \mathrm{CN}$ at $3 \times 10^{-5} \mathrm{M}$. Emission spectra were obtained by excitation at $350 \mathrm{~nm}$

Figure 17: ${ }^{1} \mathrm{H}$ NMR of (a) BT1, (b) BT2, and (c) BT3 with increasing amounts of mercury(II) in $\mathrm{CD}_{3} \mathrm{CN}$ at $4 \times 10^{-3} \mathrm{M}$ of sensor. 58

Figure 18: Competition experiments showing the difference in absorptivity for (a) BT1, (b) BT2, and (c) BT3 in the presence of 5 eq of metal salt (green bars) and subsequent 
addition of 5 eq of mercury(II) (blue bars) in $\mathrm{CH}_{3} \mathrm{CN}$ at $3 \times 10^{-5} \mathrm{M}$ concentration of sensor.

Figure 19: Structural depiction of L2 and P1-P3.

Figure 20: Absorption (a) and emission (b) spectrum of L2 and P1-P3 in $\mathrm{CH}_{3} \mathrm{CN}$ at $1 \times 10^{-5} \mathrm{M}$

Figure 21: Absorption (a) and emission (b) of P1-P3 in $\mathrm{H}_{2} \mathrm{O}$ at $1 \times 10^{-5} \mathrm{M}$ concentration $(\mathrm{pH} 7)$ 68

Figure 22: Absorption of P1 (a), P2 (c), and P3 (e) and emission of P1 (b), P2 (d), and P3 (f) in acidic (red), basic (red) or neutral (blue) conditions. Emission spectra are normalized except for P3 to highlight quenching. 70

Figure 23: Change in absorption spectrum in $\mathrm{CH}_{3} \mathrm{CN}$ of $\mathrm{P} 1$ (a-b), $\mathrm{P} 2$ (c-d), and P3 (e-f) at $1 \times 10^{-5} \mathrm{M}$ with increasing amounts of $\mathrm{Hg}\left(\mathrm{ClO}_{4}\right)_{2}$. Spectra show formation of two distinct products in solution. 73 Figure 24: Change in emission spectrum in $\mathrm{CH}_{3} \mathrm{CN}$ of P1 (a-b), P2 (c-d), and P3 (e-f) at $1 \times 10^{-5} \mathrm{M}$ with increasing amounts of $\mathrm{Hg}\left(\mathrm{ClO}_{4}\right)_{2}$. Spectra show formation of two distinct products in solution. Titrations for solutions of P1, P2 and P3 were excited at $360 \mathrm{~nm}$, $350 \mathrm{~nm}$, and $360 \mathrm{~nm}$, respectively.

Figure 25: Change in absorption spectrum of (a) P1 and (b) P3 after addition of $\mathrm{Hg}\left(\mathrm{ClO}_{4}\right)_{2}$ (blue) and subsequent addition of EDTA (green) in $\mathrm{CH}_{3} \mathrm{CN}$ at $1 \times 10^{-5} \mathrm{M} \ldots . .75$ Figure 26: Job's plots obtained for (a) P1, (b) P2 and (c) P3 with mercury(II) at $1 \times 10^{-5}$ $\mathrm{M}$ in $\mathrm{CH}_{3} \mathrm{CN}$. 
Figure 27: Absorption (a, c, \& e) and emission (b, d, \& f) titrations of P1, P2 and P3 in 50:50 $\mathrm{CH}_{3} \mathrm{CN}: \mathrm{H}_{2} \mathrm{O}$. Emission titrations are excited at and $350 \mathrm{~nm}, 400 \mathrm{~nm}$, and $360 \mathrm{~nm}$ for P1, P2 and P3 respectively. Excitation wavelengths were adjusted for solvent in order to obtain a complete spectrum and observe increasing intensity of product emission in the case of $\mathrm{P} 2$. 80

Figure 28: Change in ${ }^{1} \mathrm{H}$ NMR spectrum with increasing amounts of $\mathrm{Hg}\left(\mathrm{ClO}_{4}\right)_{2}$ for (a) P1, (b) P2, and (c) P3. A general structure for each ligand is overlaid above all three spectrum with labeled protons. The protons of the octaethylene glycol monomethyl ether chain are omitted for P2. Amine proton peaks have been intensified to make evident in (c)

Figure 29: Emission of (a) P1, (b) P2, and (c) P3 at 444, 507, and $480 \mathrm{~nm}$, respectively, after the addition of $5 \mathrm{~mol}$ eq of various metal ions as perchlorate salts (grey bars) and then subsequent addition of $5 \mathrm{~mol}$ eq of $\mathrm{Hg}\left(\mathrm{ClO}_{4}\right)_{2}$ in $\mathrm{CH}_{3} \mathrm{CN}$. Ligand emission is

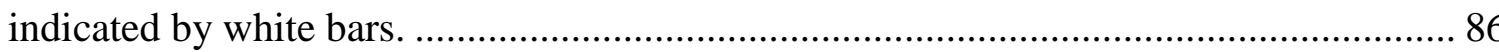

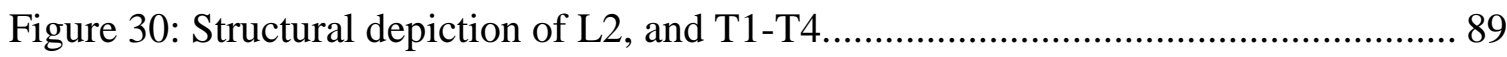

Figure 31: Examples of possible coordination complex structures with T4.................. 90 Figure 32: Absorption (a) and emission (b) of L2 and T1-T4 in $\mathrm{CH}_{3} \mathrm{CN}$. Solutions of L2,

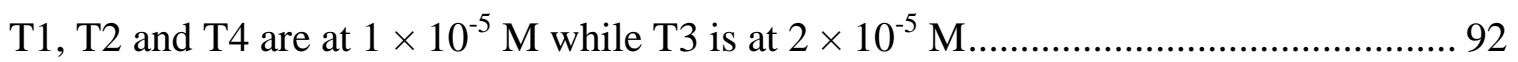
Figure 33: Optimized geometries of (a) T1 and (b) T3 determined by DFT calculations (B3LYP /6-311 + G(d)) 
Figure 34: Absorption and emission response of T1 to iron(III) (a-d) and mercury(II) (eh) in $\mathrm{CH}_{3} \mathrm{CN}$ at $1 \times 10^{-5} \mathrm{M}$. Emission spectrum were obtained by excitation at $360 \mathrm{~nm}(\mathrm{c}-$ d) and $345 \mathrm{~nm}(\mathrm{~g}-\mathrm{h})$. 96

Figure 35: Absorption and emission response of T1 to copper(II) (a-b) and lead(II) (c-d) in $\mathrm{CH}_{3} \mathrm{CN}$ at $1 \times 10^{-5} \mathrm{M}$. Emission spectrum were obtained with an excitation wavelength of $360 \mathrm{~nm}$ 97 Figure 36: ${ }^{1} \mathrm{H}$ NMR titration of T1 at $4 \times 10^{-3} \mathrm{M}$ with (a) iron(III), (c) lead(II), and (e) mercury(II) with increasing amounts of metal perchlorate salt in $\mathrm{CD}_{3} \mathrm{CN}$. The magnitude of the chemical shift is plotted for each peak with increasing equivalents of (b) iron(III), (d) lead, and mercury(II). 100

Figure 37: Absorption at the complex peak (381 nm) of T1 (white bars), after the addition of 5 eq. of metal ion (green bars), and subsequent addition of 5 eq. of either copper(II), iron(III), lead(II) or mercury(II) perchlorate (blue bars) in $\mathrm{CH}_{3} \mathrm{CN} \ldots \ldots \ldots \ldots \ldots \ldots \ldots \ldots \ldots . . . . . . . . . . . . . . .101$ Figure 38: Absorption titrations of T2 with copper(II) (a-c), iron(III) (d-f), lead(II) (g-i), and mercury(II) (j-1). 104 Figure 39: Change in emission of T2 with increasing amounts of copper(II) (a-b), iron(III) (c-d), and mercury(II) (e-f) in $\mathrm{CH}_{3} \mathrm{CN}$ at $1 \times 10^{-5} \mathrm{M}$. 105

Figure 40: ${ }^{1} \mathrm{H}$ NMR titration of T2 at $4 \times 10^{-3} \mathrm{M}$ with increasing amounts of copper(II) and iron(III) in $\mathrm{CD}_{3} \mathrm{CN}$. The magnitude of the chemical shift is plotted for each peak with increasing equivalents of (b) copper(II), and (d) iron(III). 107 
Figure 41: ${ }^{1} \mathrm{H}$ NMR titration of T2 at $4 \times 10^{-3} \mathrm{M}$ with increasing amounts of lead(II) and mercury(III) in $\mathrm{CD}_{3} \mathrm{CN}$. The magnitude of the chemical shift is plotted for each peak with

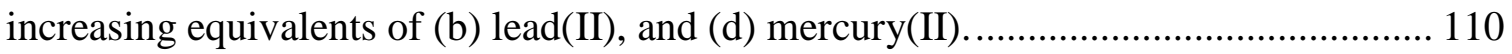
Figure 42: Absorbance Job's plots obtained with T2 and copper(II), iron(III), lead(II) and mercury(II) in $\mathrm{CH}_{3} \mathrm{CN}$ at a total concentration of $2 \times 10^{-5} \mathrm{M}$. 111 Figure 43: Absorption at 390, 422, and $399 \mathrm{~nm}$ of T2 (white bars), after the addition of 5 eq. of metal ion (green bars), and subsequent addition of 5 eq. of either copper(II), iron(III), lead(II) or mercury(II) perchlorate (blue bars) in $\mathrm{CH}_{3} \mathrm{CN}$ 114 Figure 44: Change in absorption spectrum of T3 with increasing amounts of copper(II) (a-b), iron(III) (c-d), lead(II) (e-f), and mercury(II) in $\mathrm{CH}_{3} \mathrm{CN}$ at $2 \times 10^{-5} \mathrm{M}$. 116 Figure 45: Change in emission spectrum of T3 with increasing amounts of copper(II) (ab), iron(III) (c-d), lead(II) (e-f) and mercury(II) (g-h) in $\mathrm{CH}_{3} \mathrm{CN}$ at $2 \times 10-5 \mathrm{M}$. All solutions were excited at $350 \mathrm{~nm}$ 118 Figure 46: Job's plot obtained for T3 with copper(II), iron(III), and mercury(II) in $\mathrm{CH}_{3} \mathrm{CN}$ at a total concentration of $2 \times 10^{-5} \mathrm{M}$ Figure 47: Absorbance spectrum of T3 at $2 \times 10^{-5} \mathrm{M}$ (red), T3 and 5 eq. of copper(II) (green), T3 and 5 eq. of sodium(I) (blue), and T3 with 5 eq. of both sodium(I) and copper(II) in $\mathrm{CH}_{3} \mathrm{CN}$. 121 Figure 48: ${ }^{1} \mathrm{H}$ NMR of T3 (red), T3 and 2 equivalents of sodium (green), and T3 in the presence of 2 eq of sodium and 1 eq of copper(II) (blue) in $\mathrm{CD}_{3} \mathrm{CN}$ at $4 \times 10^{-3} \mathrm{M} \ldots \ldots .122$ 
Figure 49: ${ }^{1} \mathrm{H}$ NMR titration of T3 with (a) iron(III), (b) lead(II), and (c) mercury(II) in $\mathrm{CD}_{3} \mathrm{CN}$ at $4 \times 10^{-3} \mathrm{M}$. The magnitude of the chemical shift is plotted for each peak with increasing equivalents of (b) iron(III), (d) lead(II), and (f) mercury(II) 124

Figure 50: Absorption at $386 \mathrm{~nm}$ of T3 (white bars), after the addition of 5 eq. of metal ion (green bars), and subsequent addition of 5 eq. of either copper(II), iron(III), lead(II) or mercury(II) perchlorate (blue bars) in $\mathrm{CH}_{3} \mathrm{CN}$. 125 Figure 51: Change in absorbance of T4 with increasing amounts of copper(II) (a-b), iron(III), (c-d), and lead(II) (e) in $\mathrm{CH}_{3} \mathrm{CN}$ at $1 \times 10^{-5} \mathrm{M}$. Figure 52: Change in absorption (a-c) and emission (d-f) of T4 in response to increasing amounts of mercury(II) in $\mathrm{CH}_{3} \mathrm{CN}$ at $1 \times 10^{-5} \mathrm{M}$. Emission spectra were obtained by exciting at $370 \mathrm{~nm}$ 128 Figure 53: Change in emission spectrum of T4 with increasing amounts of copper(II) (ab), iron(III) (c-d) and lead(II) (e) in $\mathrm{CH}_{3} \mathrm{CN}$ at $1 \times 10^{-5} \mathrm{M}$. All emission spectra were obtained by exciting at $370 \mathrm{~nm}$

Figure 54: Job's plots for T4 with copper(II), iron(III), lead(II) and mercury(II) in $\mathrm{CH}_{3} \mathrm{CN}$ at a total concentration of $2 \times 10^{-5} \mathrm{M}$. 131

Figure 55: ${ }^{1} \mathrm{H}$ NMR titration of T4 at $4 \times 10^{-3} \mathrm{M}$ with increasing amounts of lead(II) and mercury(II) in $\mathrm{CD}_{3} \mathrm{CN}$. The magnitude of the chemical shift is plotted for each peak with increasing equivalents of (b) lead(II), and (d) mercury(II) 133

Figure 56: ${ }^{1} \mathrm{H}$ NMR titration of T4 at $4 \times 10^{-3} \mathrm{M}$ with increasing amounts of copper(II) and iron(III) in $\mathrm{CD}_{3} \mathrm{CN}$. The magnitude of the chemical shift is plotted for each peak with increasing equivalents of (b) copper(II), and (d) iron(III). 135 
Figure 57: Absorption at $440 \mathrm{~nm}$ of T4 (white bars), after the addition of 5 eq. of metal ion (green bars), and subsequent addition of 5 eq. of either copper(II), iron(III), lead(II)

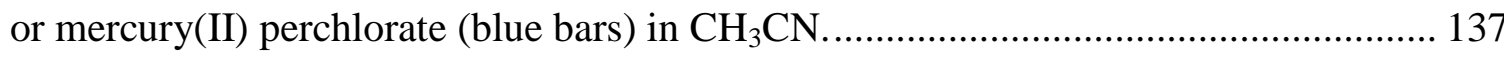




\title{
List of Symbols and Abbreviations
}

\author{
$K_{\mathrm{a}}$ Association Constant \\ EPA US Environmental Protection Agency \\ $\varepsilon$ Molar absorptivity \\ $\lambda_{\text {abs }}$ Absorption wavelength \\ $\lambda_{\mathrm{ex}}$ Excitation wavelength \\ $\lambda_{\text {em }}$ Emission wavelength \\ $\phi \quad$ Emission quantum yield \\ HSAB Hard-Soft Acid-Base theory \\ BODIPY boron-dipyrromethene \\ $\mathrm{CH}_{3} \mathrm{CN}$ Acetonitrile \\ THF Tetrahydrofuran \\ DMF Dimethylformamide \\ $\mathrm{CH}_{2} \mathrm{Cl}_{2}$ Dichloromethane \\ $\mathrm{Et}_{2} \mathrm{O}$ Diethyl ether \\ $\mathrm{CHCl}_{3}$ Chloroform \\ $\mathrm{H}_{2} \mathrm{O}$ Water \\ EtOAc Ethyl acetate \\ $\mathrm{MeOH}$ Methanol \\ EtOH Ethanol \\ TEA Triethylamine \\ $\mathrm{Pd}\left(\mathrm{PPh}_{3}\right)_{4} \quad$ tetrakis(triphenylphosphine)-palladium(0)
}


$\mathrm{Na}_{2} \mathrm{SO}_{4}$ Sodium sulfate

$\mathrm{PdCl}_{2}\left(\mathrm{PPh}_{3}\right)_{2} \quad$ Bis(triphenylphosphine)palladium(II) dichloride

$\mathrm{HCl}$ Hydrochloric acid

$\mathrm{NaOH}$ Sodium hydroxide

$\mathrm{NaHCO}_{3}$ Sodium bicarbonate

$\mathrm{NaCl}$ Sodium chloride

EDTA Ethylenediaminetetraacetic acid

$\mathrm{N}_{2} \quad$ Nitrogen gas

$\mathrm{CDCl}_{3}$ Deuterated chloroform

MeOD Deuterated methanol

$\mathrm{CD}_{3} \mathrm{CN}$ Deuterated acetonitrile

DFT Density functional theory

L1 2-(2'-thienyl)-pyridine

L2 2,5-bis(2-pyridyl)thiophene

L3 2,6-bis(2-thienyl)pyridine

BT1 2-(dibenzo[b,d]thiophen-4-yl)pyridine

BT2 2-(dibenzo[b,d]thiophen-4-yl)-1H-imidazole

BT3 2-(dibenzo[b,d]thiophen-4-yl)thiazole

P1 6,6'-thiophene-2,5-diyl)bis(pyridine-3-ol)

P2 2,5-bis(5-((2,5,8,11,14,17,20-heptaoxadocosan-22-yl)oxy)pyridine-2yl)thiophene

P3 6,6'-(thiophene-2,5-diyl)bis(pyridine-3-amine)

T1 2,2'-(3,4-dimethoxythiophene-2,5-diyl)dipyridine 
T2 2,5-di(pyridin-2-yl)thiophene-3,4-diol

T3 2,2'-(3,4-bis((2,5,8,11,14,17,20-heptaoxadocosan-22-yl)oxy)thiophene2,5-diyl)dipyridine

T4 2,5-di(pyridin-2-yl)thiophene-3,4-diamine 


\section{Chapter 1. Introduction}

Industrialization across the globe has led to an increase in the use of metals, and consequently, exposure to toxic metals. ${ }^{1-3}$ The outbreak of methylmercury poisoning in Minamata city ${ }^{4}$ is one example of a population being exposed to toxic metals in the environment and the serious consequences that come with it. In order to prevent incidents such as these, researchers have developed methods for the selective detection of metals in the environment. In addition to detecting metals in the environment there is also a need to better understand their activity in biological systems, requiring detectors for metal ions for in vivo studies as well..$^{5-7}$ Common methods for detecting and quantifying metal ions are atomic absorption spectroscopy and inductively coupled plasma mass

spectrometry. ${ }^{8-10}$ Though these methods are capable of detecting minute concentrations of a given metal they require extensive sample preparation and are costly to perform. Furthermore the bulky instruments that these methods require do not lend themselves to field-testing, requiring samples to be sent to a lab for analysis. Fluorescent and colorimetric sensors are attractive alternatives to these methods as they are both costeffective and portable. ${ }^{6,8,9}$

Herein the metal ions of interest to this work will be discussed and an overview of the benefit to developing sensors for them will be provided. Following this the relevant characteristics to consider when developing fluorescent and colorimetric sensors for metal ions will be reviewed in addition to several strategies for improving these properties. This introduction will conclude with a discussion of thiophene coordination 
chemistry and the applications of thiophene in molecular sensors, in the context of the work described in later chapters.

\subsection{Metal Ions of Interest}

Generally speaking the metal ions of interest to researchers are those which are biologically relevant and those which are toxic. Of the metals that are biologically relevant there are some which can cause severe negative effects when there is an excess or deficiency in the body, such as copper(II) and iron(III). ${ }^{5}$ In contrast heavy metals, such as mercury(II) and lead(II), are dangerous to life at even minute concentrations. ${ }^{5}$ All of these metals at one point, have found applications around the globe resulting in the release of these metals into a variety of environments and consequently exposure to human populations.

Iron, as one of the most abundant metals on earth, has found widespread use in applications in metallurgy for the production of steel and other alloys, or as catalysts for the production of ammonia in the Haber-Bosch process. This metal is used ubiquitously as it is considered relatively nontoxic and known to be vital to life. However at high concentrations iron(III) can produce destructive oxygen species such as hydroxyl radicals via the Fenton reaction. ${ }^{5,11}$ These potentially dangerous side reactions limit the tolerable range of iron(III) in the body and provide the impetus to develop a method of detection for this metal. ${ }^{11-13}$ The low solubility of iron(III) compounds in water at neutral $\mathrm{pH}$, however, limits our exposure to this metal from drinking water. Therefore our primary source of iron(III) results from the consumption of food products or dietary supplements. 
Therefore rather than detecting this metal in water researchers focus on designing sensors for identifying this metal in food products and observing the activity of iron(III) in biological systems.

Copper is a metal, similar to iron, that has found widespread use for applications as a conductor in wiring as well as other applications. Though vital to the body for its role in oxygen transport, copper(II) can induce a number of detrimental side-effects at high concentrations such as neurodegeneration, Wilson's disease, and the development of Parkinsonism. ${ }^{13-15}$ Furthermore excess copper(II) in the body has been linked to Alzheimer's disease, ${ }^{14-17}$ providing greater impetus to study this metals activity at high concentrations in vivo. Copper mining and pollution have resulted in an increase in concentration of this metal in the environment, requiring a limit be set on the amount of copper(II) in drinking water to be less than $1.3 \mathrm{ppm}$ according to the US Environmental Protection Agency (EPA). ${ }^{18}$

In contrast to biologically relevant molecules, heavy metals are very toxic at even minute concentrations. As such researchers are primarily interested in developing cheaper and more portable methodologies for the detection of these metals in the environment to prevent human exposure. However, biological sensors for these metals are desired as well in order to better understand the toxic nature of these heavy metals in vivo. ${ }^{5}$ Lead(II) has been used for a variety of applications such as batteries, paints, additives in fuel, and as an alloy in pipes. Since lead(II) is a known neurotoxin that can result in a number of neurological and developmental disorders, ${ }^{2,5,19}$ it has been gradually removed from use 
for these applications. In accordance with the danger this metal poses the EPA has set the limit of lead(II) in drinking water to be no more than $15 \mathrm{ppb} .^{18}$

Mercury(II) is another toxic heavy metal which can cause a number of neurological and developmental disorders, similar to lead. ${ }^{1,5}$ Mercury was formerly used in appliances such as thermometers and as catalysts for the production of organic compounds such as acetaldehyde. The use of mercury(II) catalysts for the production of acetaldehyde ultimately led to the outbreak of methylmercury poisoning in Minamata city. Though in the case of Minamata city the exposure to methylmercury was due to improper disposal of chemical waste from a local industry, methylmercury can form in the environment from elemental mercury or mercury(II) compounds. In addition to the toxic nature of this metal, mercury(II) is known to accumulate within the body upon repeated exposure further adding to the dangerous nature of this metal. To limit the amount of exposure to mercury(II) the EPA has set the limit allowed in drinking water to 2 ppb. ${ }^{20}$

The initial work on this project focused on the development of a mercury(II) sensor using thiophene-based fluorescent-sensors. Though a majority of the sensors to be discussed had a response to mercury(II), some of these ligands were capable of binding to other metals as well including: copper(II), iron(III), and lead(II). As such later work focused on the development of thiophene-based multiplex sensors, covered in Chapter 6 as defined and discussed in section 1.2.2 of this Introduction. The primary focus of the work discussed in this document was the development of fluorescent and colorimetric thiophene-based mercury(II) sensors. 


\subsection{Designing Fluorescent and Colorimetric Sensors}

In the context of this work, a fluorescent and colorimetric sensor is considered to be a ligand which binds to the metal analyte resulting in a metal complex. The metal complex formed will have different photophysical properties relative to the ligand, typically emission or absorbance, which can be measured and used to quantify the concentration of metal. The design of fluorescent and colorimetric sensors generally involves two major considerations: selectivity and sensitivity. A sensor should be both selective to the target analyte against other potential ions in solution, and should also be sensitive enough to detect minute concentrations of that analyte. Perhaps the most relevant factor in determining the chemical sensors sensitivity and selectivity is the association constant $\left(K_{\mathrm{a}}\right)$ for the metal analyte of choice. Generally speaking a sensor should bind preferentially to a metal that it has the highest $K_{\mathrm{a}}$ for, against other metal ions. The $K_{\mathrm{a}}$ will also dictate the sensors sensitivity to that metal, as the greater the magnitude of the $K_{\mathrm{a}}$ the lower the amounts of metal required to form the resulting metal complex. Thus a sensor that has a high $K_{\mathrm{a}}$ will have a lower limit of detection for that metal ion, relative to a sensor with a smaller $K_{\mathrm{a}}$. In addition to the $K_{\mathrm{a}}$ there are several photophysical characteristics which should be considered including: molar absorptivity ( $\varepsilon)$, absorption wavelength $\left(\lambda_{\mathrm{abs}}\right)$, excitation wavelength $\left(\lambda_{\mathrm{ex}}\right)$, emission wavelength $\left(\lambda_{\mathrm{em}}\right)$, and emission quantum yield $(\phi)$.

\subsubsection{The Association Constant and Strategies for the Design of Chemical Sensors}




$$
\mathrm{aM}+\mathrm{bL} \rightleftharpoons \mathrm{M}_{\mathrm{a}} \mathrm{L}_{\mathrm{b}}
$$

Scheme 1: General equilibrium expression of a ligand binding to a metal center.

Before discussing strategies to design a sensor with a high $K_{\mathrm{a}}$ value, it is beneficial to define $K_{\mathrm{a}}$. First we describe the system in the context of the general equilibrium equation given by Scheme 1 where a ligand (L) binds to a metal (M) to form a metal complex $\left(\mathrm{M}_{\mathrm{a}} \mathrm{L}_{\mathrm{b}}\right)$ at a given stoichiometric ratio (a:b). The $K_{\mathrm{a}}$ of any ligand or sensor can then be defined by Equation 1, where $[M],[L]$ and $\left[M_{a} L_{b}\right]$ are the concentrations of the metal, ligand and metal complex, respectively, at equilibrium. As a thermodynamic

constant, $K_{\mathrm{a}}$ is directly related to the stability of the formed complex. Thus in order to develop a sensor with a high $K_{\mathrm{a}}$ it is necessary to consider the thermodynamic stability of the formed complex.

\section{Equation 1}

$$
K_{a}=\frac{\left[M_{a} L_{b}\right]}{[M]^{a}[L]^{b}}
$$

There are several strategies that can be utilized to design a molecular sensor that will form a thermodynamically stable complex with the metal ion of interest. Researchers typically rely on Hard-Soft Acid-Base theory (HSAB) for the design of chemical sensors. This concept defines a Lewis acid and base to be either 'hard' or 'soft.' A hard Lewis acid is a small, weakly polarizable species with a high oxidation state and a soft Lewis acid is a large, strongly polarizable species with a low oxidation state. Hard and soft Lewis bases are similar to the corresponding Lewis acids, except these are generally 
neutral or have a negative charge. According to HSAB theory, hard Lewis bases form more stable bonds with hard Lewis acids, and soft Lewis bases do the same with soft Lewis acids. Therefore in order to design a sensor to have a high $K_{\mathrm{a}}$, researchers strategically choose soft or hard Lewis bases for soft or hard Lewis acids, respectively.

(a)

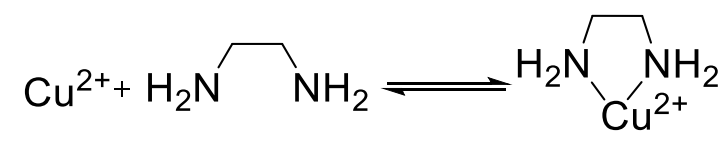

(b)

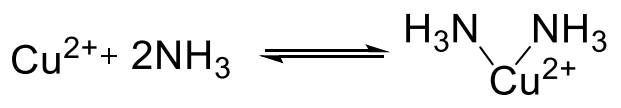

Scheme 2: Equilibrium expression for the binding of ethylenediamine (a) and ammonia (b) to a copper(II) metal center. Other donor atoms are omitted for clarity.

In addition to $\mathrm{HSAB}$, the denticity of the sensor can be utilized to design a sensor with an ideal $K_{\mathrm{a}}$. The denticity of a ligand refers to the number of donor atoms that bind to a metal ion. Generally the greater number of donor atoms increases the $K_{\mathrm{a}}$, which can be explained in terms of entropy. If one compares the change in entropy from the two equilibrium reactions in Scheme 2 it is evident there will be a greater decrease in entropy when two moles of ammonia bind to copper(II) rather than one mole of ethylenediamine. A larger decrease in entropy translates to a more thermodynamically unstable complex, and therefore a lower $K_{\mathrm{a}}$ relative to the ethylenediamine complex. Researchers, therefore typically design molecular sensors to include multiple donor atoms to form more thermodynamically stable complexes.

When implementing chelating groups the chelate ring effect is an important consideration for the design of sensors. The spacing between each donor atom affects 
what is known as the chelate ring size. For example, the ethylenediamine complex shown in Scheme 2 is a 5 -membered chelate ring. The size of the chelate ring will influence the $K_{\mathrm{a}}$ of the formed complex, where larger chelate rings generally form more stable complexes with small metal ions and small chelate rings form stable metal complexes with large metal ions. ${ }^{21,22}$ Generally speaking smaller chelate rings can only be between four or five atoms, as rings smaller than this are too sterically strained to be stable. Large chelate rings are therefore anything larger than five, though typically this does not exceed six atoms. Careful modification of the chelate ring size can thus allow for the strategic design of a sensor that can form a stable complex to a given metal ion of a given ionic radius.

Though in general researchers are interested in developing chemical sensors with a high affinity for the metal ion of interest, it is worth noting here that a reversible response of the sensor is still desired. In the context of environmental sensors, reversibility is desired for recycling of the sensor for later use or for dynamic sensing. When considering chemical sensors for biological systems the reversibility of the sensor is even more relevant. The concentration of free metal ion in vivo is constantly in flux, thus it is necessary for a sensor to be capable of releasing the bound metal to ensure researchers are able to monitor the change over time. This way it is possible to develop chemical sensors capable of yielding significantly more information, compared to detectors which can only provide single-measurements.

\subsubsection{Ratiometric, Multiplex and Fluorescent Sensors}


The primary focus of this section will be to define some of the various classifications that chemical sensors can fall under, such as fluorescent and colorimetric sensors as well as ratiometric and multiplex sensors. In addition to defining the various sensor types the advantages and disadvantages of each type will be reviewed. Strategies used to design sensors of a given type will be discussed followed by a brief review of applications for both fluorescent and colorimetric sensors.

(a)

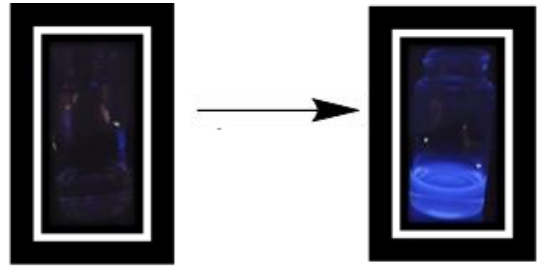

(b)

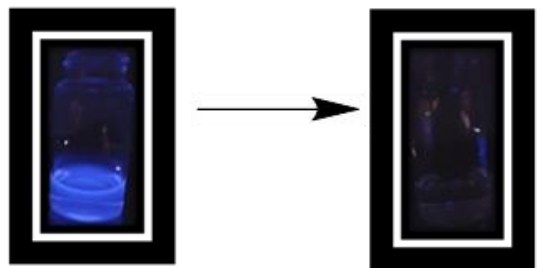

(c)

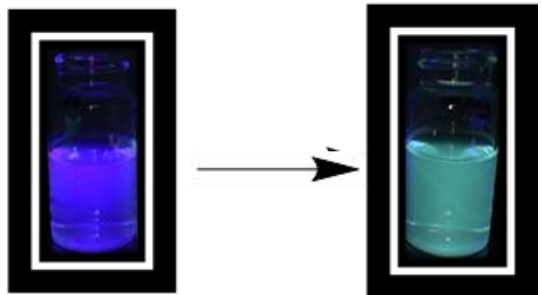

Figure 1: Visual represntation of (a) 'turn-on' sensor, (b) 'turn-off' sensor, and (c) ratiometric sensor. Images obtained from sensors that are discussed later in this document.

Fluorescent molecular sensors may be designated as either 'turn-on,' 'turn-off' or ratiometric sensors (Figure 1). 'Turn-on' sensors are molecules which have either an enhanced fluorescence, or become fluorescent in response to the analyte. A 'turn-off' sensor is a molecule whose fluorescence is quenched in response to a given analyte. 
'Turn-on' sensors are more preferred as they are less prone to false positive readings. Quantification of a metal analyte with 'turn-on' and 'turn-off' sensors may be done by measuring the amount of light emitted by the sensor. Researchers often utilize modifiedrhodamine type dyes to develop 'turn-on' sensors, as these molecules have both nonfluorescent spirolactam ('ring-closed') form and the fluorescent 'ring-open' form. ${ }^{23}$ Binding of a metal to donor atoms cause the formation of the 'ring-open' form, resulting in a fluorescent species, and 'turn-on' detection (Scheme 3). Other similar types of sensors involve conformational or structural change that result in the ligand transitioning between a nonfluorescent to fluorescent structure. ${ }^{8,24,25}$

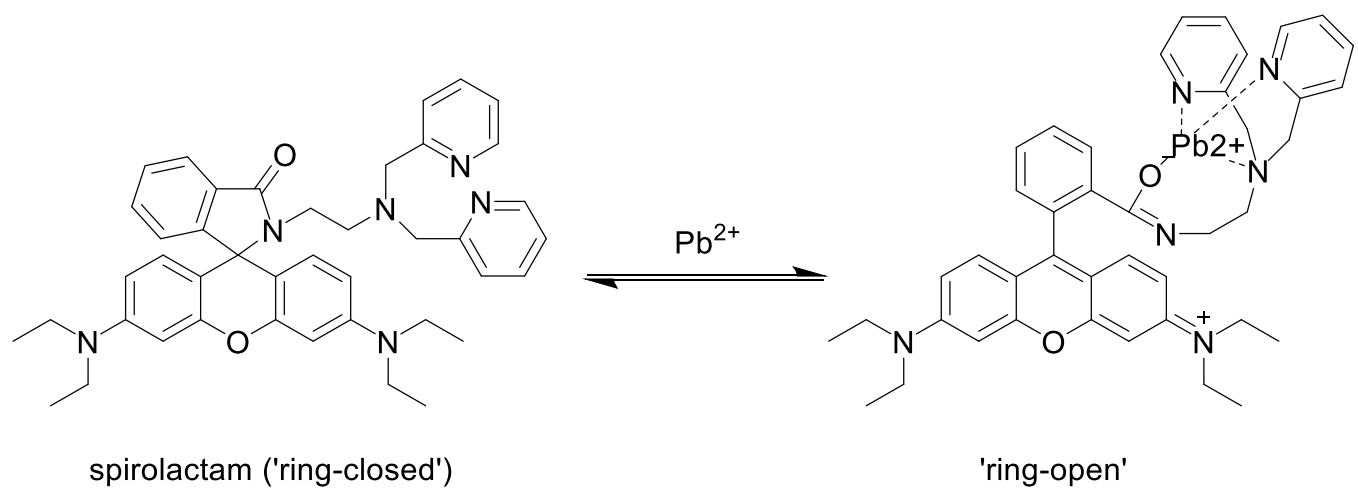

Scheme 3: An example of a rhodamine 'turn-on' sensor in both the spirolactam and 'ring-open' form upon binding to lead(II). ${ }^{23}$

Though 'turn-on' sensors can be used for the measurement of a given metal analyte in solution, ratiometric sensors are the preferred method of quantification. Ratiometric sensing refers to the detection of a given analyte by measuring the ratio of absorption or emission intensities at two separate wavelengths. ${ }^{26}$ By measuring the ratio at two wavelengths the measurement is normalized and quantification can be achieved through a 
simple calibration curve. It should be noted here that though ratiometric sensing is typically discussed in the context of fluorescent sensing, ratiometric absorbance sensing is possible as well. Ratiometric sensors are particularly advantageous for in vivo measurements, since the measurement is normalized against another wavelength, accurate values can be obtained regardless of differences in cell thickness. ${ }^{26}$ In addition to sensing in biological systems, ratiometric sensors are ideal for environmental samples. Since the measurement is normalized, the obtained concentrations are less prone to artifacts of the instrument, allowing for more accurate measurements. Though ratiometric sensing is highly desired there are no known strategies to design ratiometric sensors. Rhodamine dyes have been shown to be capable of ratiometric sensing, ${ }^{27,28}$ though this is not a general strategy for the development of a ratiometric sensor.

The fluorescent and colorimetric sensors that have been discussed so far are designed for targeting a single analyte, however multiplex sensors are designed to simultaneously detect multiple analytes instead. ${ }^{29-33}$ Multiplex sensing is particularly advantageous for both biological and environmental applications as it allows for the detection of several analytes at once with the use of a single sensor. Examination of various analytes in a single measurement can save both time and resources. A multiplex sensor should be capable of providing a unique response for each of the potential analytes. This means that the resulting metal complex formed with the sensor should have a different $\lambda_{\max }$ dependent on the metal ion the ligand is coordinated to. The few examples of this class of sensor that exist in the literature utilize DNA-functionalized nanoparticles of various sizes, or are large molecular sensors with multiple coordination 
modes that bind to different metals selectively. ${ }^{34-40}$ Multiplex sensors that have been previously reported incorporate rather large molecules which are not conducive to cell permeation. This presents a challenge for in vivo studies, thus providing the impetus to develop small-molecule multiplex sensors.

\subsubsection{Characteristics to Consider in the Design of Fluorescent and Colorimeteric Molecular Sensors}

Fluorescent sensors are ideal for the study of metal concentration in vivo and in vitro as fluorescence can be more readily visualized and measured in cellular media compared to absorbance. The $\mathrm{pH}$ sensitivity, cell permeability, excitation wavelength, emission wavelength, and toxicity of the sensor should be addressed. The variation in $\mathrm{pH}$ in different biological environments requires that these sensors operate across a range of $\mathrm{pH}$ values. In order to be applicable for in vivo and in vitro imaging these sensors must also be capable of permeating cells. Generally, excitation wavelengths should be lower in energy to avoid damaging the cell and to allow for penetration of light into the system of interest. Ideally these wavelengths should be far red-shifted in the visible region to the near-IR region. Consequently, low energy emission wavelengths are also desirable, though generally speaking if a compound has a low energy excitation the emission wavelength is also low in energy. Excitation and emission wavelengths can be tuned to some degree via extension of conjugated systems in organic molecules and functionalization of an aromatic ring. ${ }^{41}$ In addition to these factors, quantum yield of the fluorescent sensor should be considered as well. Quantum yield is defined as the ratio of photons emitted to photons absorbed by the fluorescent molecule. Fluorescent sensors 
with high quantum yields are desired as this allows for greater resolution of the fluorescent imaging, though this is not strictly required.

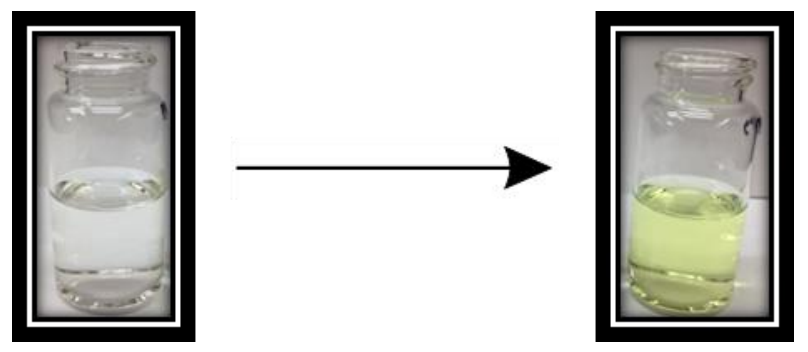

Figure 2: Visual representation of a colorimetric sensor changing color in response to a metal analyte.

While fluorescent sensors are a useful tool for the study of biological systems, colorimetric sensors are useful for the study and measurement of metal ions in the environment. Colorimetric sensors are more advantageous for this application as they do not require excitation via a secondary light source to obtain a measurement. Instead these sensors could be applied to a test strip, similar to that of $\mathrm{pH}$ paper, or incorporated in a handheld colorimeter device for measurement of the exact concentration. Colorimetric sensors that have absorbance values in the visible region are applicable in real-world settings since it allows detection of the metal of interest via simple visible inspection (Figure 2). Furthermore, high absorptivity values are desired such that low concentrations of the sensor can be used and still allow for visualization in solution or on a test strip. In addition to these factors it is also useful for the sensors to be applicable across a range of $\mathrm{pH}$ values, as environmental samples can have varied $\mathrm{pH}$ depending on the location. 


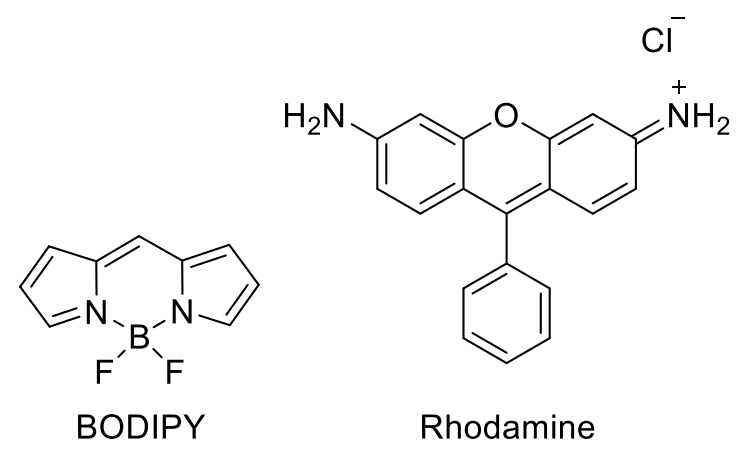

Figure 3: Structural depiction of BODIPY (left) and rhodamine (right) dyes.

Currently many molecular sensors which exist for metal ions are based on previously established architectures such as BODIPY or rhodamine type dyes (Figure 3). ${ }^{8,9,42,43}$ Though these molecular sensors provide a scaffold for researchers to modify for further improvements, they involve large molecules which are not ideal for cell permeability. In this document I will discuss the work I have done on the development of several thiophene-based small-molecule sensors. The soft sulfur atom of thiophene has been incorporated in a molecular sensor for the selective detection of soft Lewis acids such as mercury(II). In the next section a review of the work that has been done on thiophene-coordination chemistry and discuss previous examples where thiophene has been incorporated in chemical sensing systems will be provided.

\subsection{Coordination Chemistry of Thiophene and Applications as a Molecular Sensor}

Thiophene has scarcely been implemented in sensor systems for the detection of metals, as relatively little is understood about the coordination chemistry of thiophene. Previous examples of thiophene being implemented in a molecular sensor have been for the detection of a variety of metals including copper(II), iron(III) and mercury(II) (Figure 
4). ${ }^{32,44-51}$ Thiophene is advantageous in a sensor system as compounds containing this moiety are typically emissive. The emission of thiophene-based compounds may be tuned by functionalization or extension of the conjugation through the addition of aromatic systems. $^{44,52}$ This can be exploited in fluorescent and colorimetric sensors by coupling a thiophene moiety to an aromatic system to red-shift the absorption, excitation, and emission wavelengths towards the visible region. This allows for detection of a given metal by visible inspection. In addition to the fluorescent properties, the thiophene component may offer selectivity for soft Lewis acids such as mercury(II) or lead(II) due to the soft sulfur atom.
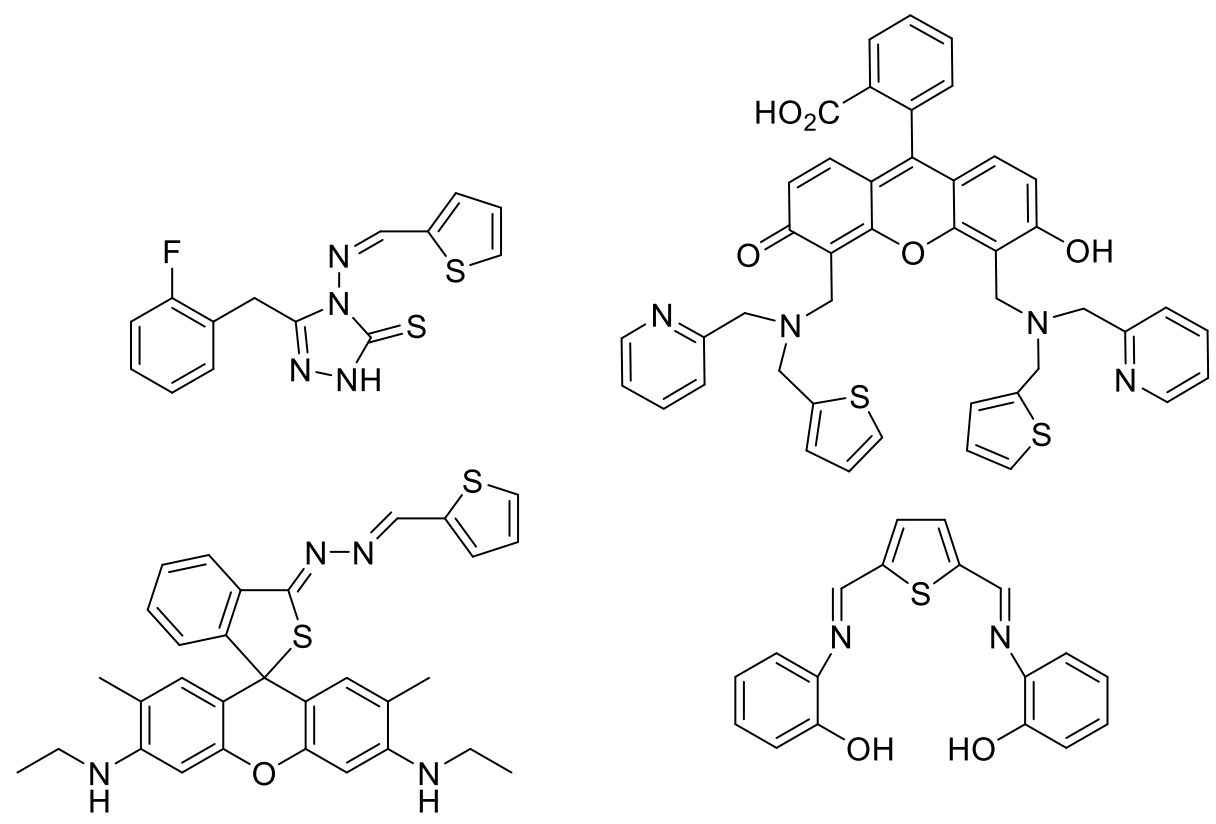

Figure 4: Structural representation of previous molecular sensors incorporating a thienyl moiety. $32,46,48,51$

Many molecular sensors have been designed with sulfur-containing functional groups, such as thiol groups, for the selective identification of soft Lewis acids like 
mercury(II) and lead(II). ${ }^{8,9,42}$ In particular modified rhodamine-type dyes containing thiol groups have been popular towards making selective fluorescent molecular "turn-on" sensors for soft Lewis acids. ${ }^{9,23,27,53}$ Some examples of these rhodamine dyes have also incorporated thiophene moieties, however there is little experimental evidence to suggest that the observed selectivity is due to the thiophene moiety. ${ }^{48,50}$ Furthermore it is unclear whether thiophene can be a useful component in a molecular sensor, as its coordination chemistry is poorly understood.

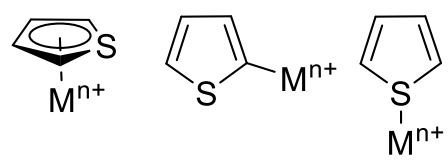

Figure 5: Structural depiction of the possible coordination modes from left to right: $\eta^{5}$ coordination via all 5 atoms, $\eta^{1}$ carbon-coordination, and $\eta^{1}$ sulfur-coordination. ${ }^{54}$

Previous work on thiophene coordination chemistry has focused on hydrodesulfurization of thiophene with transition metal catalysts. ${ }^{54}$ Among the various coordination modes that have been observed in previous work carbon-coordination is the most common, ${ }^{55,56,65-67,57-64}$ while sulfur-coordination is rarely observed (Figure 5). ${ }^{68-71}$ This is likely due to resonance effects which delocalize a negative charge along the carbon atoms and place a positive charge on the sulfur atom. Several strategies have proved effective to enhance the sulfur-binding affinity of thiophene. Chelating groups at the 2,5-positions of thiophene have been implemented to improve the likelihood of sulfur-coordination with success. ${ }^{69}$ The addition of electron-donor groups to thiophene has also been shown to increase sulfur-coordination and improve the binding affinity. ${ }^{54,68,72}$ Finally benzothiophene and dibenzothiophene have also been observed to 
have higher sulfur-binding affinities as indicated by higher equilibrium constants (Table 1)..$^{59,68,70,72-74}$ These strategies have been employed to develop thiophene-based fluorescent mercury sensors presented herein.

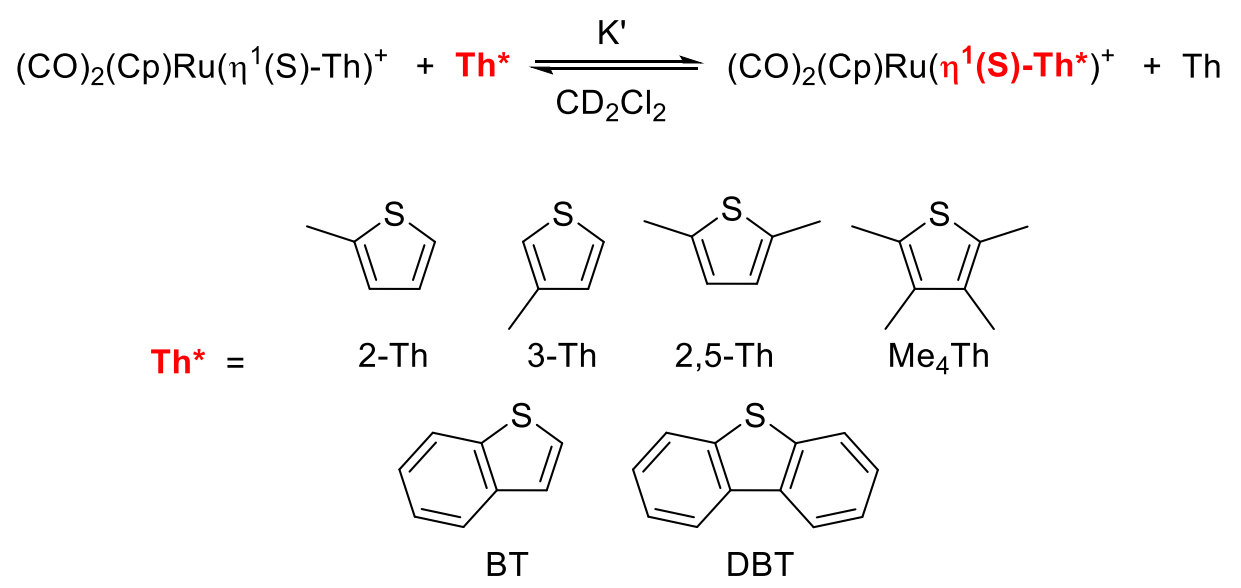

Figure 6: Displacement equilibrium expression of an $\eta^{1}$ sulfur-coordinated thiophene by a modified thiophene ligand, identified by $T{ }^{*}{ }^{72}$

Table 1: Relative equilibrium constants of various $\eta^{1}$ sulfur-coordinated thiophene ligands compared to the nonfunctionalized thiophene, for the equilibrium described in Figure $6 .{ }^{72}$

\begin{tabular}{lllllll}
\hline & 2-Th & 3-Th & 2,5-Th & Me $\mathbf{4 h}$ & BT & DBT \\
\hline K' & 4.11 & 6.30 & 2.76 & 57.4 & 29.9 & 74.1 \\
\hline
\end{tabular}

\subsection{Summary of Work}

Herein I report on thiophene-based fluorescent molecules and their response to metal ions. In addition to describing the responses observed, an overview on the change 
in supramolecular properties, such as binding stoichioemtry and $K_{\mathrm{a}}$, upon modification of the sensor will be discussed. Several thiophene-based small molecule sensors for the detection of mercury(II) have been developed. Some of these sensors have also shown specific responses to copper(II), iron(III) and lead(II) that could be employed as singlemolecule multiplex sensors. The first generation of sensors will be covered in Chapter 3 , where the goal was to determine if pyridyl chelating groups would promote sulfurcoordination of thiophene in the three ligands that were synthesized. Furthermore the influence the thiophene-moiety had on selectivity for the mercury(II) ion will be reviewed. From this project it was discovered that chelation of the pyridyl and thienyl units allowed for a general selective response to mercury(II), however the obtained $K_{\mathrm{a}}$ values were less than ideal to be applied for real-world samples.

The primary drawback to the sensors from Chapter 3 were the generally low $K_{\mathrm{a}}$ values obtained. In order to improve this several modifications to the existing sensors were made by substitution of the thiophene unit for a dibenzothiophene ligand, with the intent of increasing the low $K_{\mathrm{a}}$ values observed. This work will be discussed in Chapter 4 of this document. Though some improvements were observed with the dibenzothiophene ligand, pyridine was determined to be the most ideal chelating group from this project. While the dibenzothiophene group was determined to improve the overall $K_{\mathrm{a}}$, relative to thiophene the increase in chelate ring size was not ideal for selectivity purposes. Furthermore, implementation of the dibenzothiophene ring in fact blue-shifted the photophysical properties, to less than ideal wavelengths for sensing purposes. In order to further optimize the design the effects of functionalization on both the pyridine and 
thiophene ring were investigated, with the intent of increasing the $K_{\mathrm{a}}$ as well as improving water solubility.

The work described in Chapter 5 of this document will cover the effects of functionalizing the pyridine ring for one of the sensors from Chapter 3. Functionalization of the pyridine ring with electron donor groups was shown to improve the $K_{\mathrm{a}}$ of the ligands relative to nonfunctionalized ligand. In addition to improving the $K_{\mathrm{a}}$ the binding stoichiometry was observed to change in one instance. Though functionalization was shown to improve the $K_{\mathrm{a}}$ of these ligands for mercury(II) it also increased the affinity for various other metals as well resulting in competitive binding from other metals including copper(II) and iron(III). The water-solubilizing functional groups facilitated detection of mercury(II) in water, though it was determined that sensing in aqueous media was far from ideal. In order to further improve this design I sought to functionalize the thiophene ring, which will be discussed in the final chapter of this document (Chapter 6).

Functionalization of the thiophene ring created the possibility of various coordination modes for different metals. As various coordination modes were possible the ability of these ligands to act as single-molecule multiplex sensors was investigated, since different coordination modes should illicit a different photophysical response. The metals tested for this study included: iron(III), copper(II), lead(II) and mercury(II). Several of these ligands were shown to have a specific response to 2-4 metals in solution, suggesting they were capable of acting as multiplex sensors. In addition to this the $K_{\mathrm{a}}$ was shown to improve. 


\section{Chapter 2. Experimental Methods}

\subsection{Materials and Methods}

Reagents were obtained from Sigma Aldrich, Fisher Scientific and TCI Chemicals and used without further purification unless otherwise indicated. The ligand 2-(2'-thienyl)-pyridine (L1) was obtained from TCI Chemicals and further purified by recrystallization in hexanes. The compounds 2-(trimethylstannyl)-pyridine, 2,5-dibromo-3,4-dimethoxythiophene, and iodoheptaethylene glycol monomethyl ether were synthesized in lab following literature procedures. ${ }^{75-78}$ Acetonitrile $\left(\mathrm{CH}_{3} \mathrm{CN}\right)$, acetone, hexanes, toluene, dimethoxyethane, tetrahydrofuran (THF), dimethylformamide (DMF), dichlormethane $\left(\mathrm{CH}_{2} \mathrm{Cl}_{2}\right)$, diethyl ether $\left(\mathrm{Et}_{2} \mathrm{O}\right)$, chloroform $\left(\mathrm{CHCl}_{3}\right)$, ethyl acetate (EtOAc), methanol $(\mathrm{MeOH})$ and metal salts were obtained from Sigma Aldrich. 1,4-Dioxane was obtained from TCI chemicals. Dry solvents were prepared over molecular sieves the night prior to a reaction. Degassed solvents were purged of air and oxygen via three freeze-pump-thaw cycles unless otherwise indicated. ${ }^{1} \mathrm{H}$ NMR spectra were on a Bruker-Avance II or III spectrometer equipped with a 9.4 or 14.1 Tesla magnet operating at 400.13 or $600.13 \mathrm{MHz}$, respectively. IR spectrum were obtained using a Thermo Fisher Nicolet iS5 IR spectrometer. Mass Spectra were recorded on a ThermoElectron LTQ-Orbitrap Discovery high resolution mass spectrometer. Emission spectra, excited state lifetimes, and quantum yields were obtained using a PTI Quantamax 300 Phosphorimeter with a Xe-flash lamp and PMT detector. Quantum yields were obtained using a PTI K-sphere 'petite' integrating sphere. Absorption spectra were 
collected on a UV-3600 Shimadzu UV-Vis NIR spectrophotometer. Binding constants for compounds were determined using Thordarson's online fitting program from absorption titration data. ${ }^{79}$

\subsection{Synthesis of Sensors}

\subsubsection{Preparation of 2,5-bis(2-pyridyl)thiophene (L2)}

A flask containing 2,5-thiophenediylbisboronic acid (0.402 g, $2.33 \mathrm{mmol})$, cesium carbonate $(1.63 \mathrm{~g}, 4.99 \mathrm{mmol})$, and $\mathrm{Pd}\left(\mathrm{PPh}_{3}\right)_{4}(0.020 \mathrm{~g}, 0.017 \mathrm{mmol})$ was purged with $\mathrm{N}_{2}$ gas before adding a degassed solution of 2-bromopyridine $(0.500 \mathrm{~mL}, 5.24 \mathrm{mmol})$ in 1,4-dioxane $(10 \mathrm{~mL})$, and water $(5 \mathrm{~mL})$. The yellow solution was heated to $105^{\circ} \mathrm{C}$ and left stirring overnight. After the reaction cooled to room temperature $10 \mathrm{~mL}$ of water was added. The product was extracted in $\mathrm{CH}_{2} \mathrm{Cl}_{2}(3 \times 40 \mathrm{~mL})$. The organic layers were combined and dried with $\mathrm{Na}_{2} \mathrm{SO}_{4}$. The filtered solvent was evaporated in vacuo to afford a yellow-orange solid. The title compound was purified by silica gel column chromatography, eluted with 50:50 ethyl acetate:hexanes. The final product was obtained as a yellow solid $(0.207 \mathrm{~g}, 37.1 \%$ yield $) .{ }^{1} \mathrm{H} \mathrm{NMR}\left(\mathrm{CD}_{3} \mathrm{Cl} 400 \mathrm{MHz}\right) \delta(\mathrm{ppm}): \delta 8.60(\mathrm{~d}$, $\mathrm{J}=4.0,1 \mathrm{H}), 7.74-7.65(\mathrm{~m}, 2 \mathrm{H}), 7.64(\mathrm{~s}, 1 \mathrm{H}), 7.15-7.18(\mathrm{dd}, \mathrm{J}=6.4,4.9 \mathrm{~Hz}, 1 \mathrm{H}){ }^{13} \mathrm{C}$ NMR $\left(100 \mathrm{MHz}, \mathrm{CD}_{3} \mathrm{CN}\right) \delta 151.85,149.20,146.03,136.63,125.47,122.16,118.44$. FTMS + p ESI calcd for $\mathrm{C}_{14} \mathrm{H}_{10} \mathrm{~N}_{2} \mathrm{~S}, 239.06374$; found, 239.06560 .

\subsubsection{Synthesis of 2,6-bis(2-thienyl)pyridine (L3)}


A flask containing 2,6-dibromopyridine $(0.482 \mathrm{~g}, 2.03 \mathrm{mmol})$, thiophene-2boronic pinacol ester $(0.882 \mathrm{~g}, 4.19 \mathrm{mmol})$, potassium fluoride dihydrate $(0.351 \mathrm{~g}$, $6.04 \mathrm{mmol})$, and $\mathrm{Pd}\left(\mathrm{PPh}_{3}\right)_{4}(0.082 \mathrm{~g}, 0.064 \mathrm{mmol})$ was purged with $\mathrm{N}_{2}$. A degassed mixture of 1,2-dimethoxyethane $(20 \mathrm{~mL})$ and water $(15 \mathrm{~mL})$ was subsequently added and heated under reflux for 4 hours. Solvent was then removed in vacuo and $50 \mathrm{~mL}$ of water was added. The product was extracted in $\mathrm{Et}_{2} \mathrm{O}(3 \times 50 \mathrm{~mL})$, the organic layers combined, and then washed with water $(1 \times 50 \mathrm{~mL})$. The organic layer was dried over $\mathrm{Na}_{2} \mathrm{SO}_{4}$ and the solvent, filtered, and removed under vacuum to afford an orange oil. The residue was purified by column chromatography over silica gel $\left(50: 50 \mathrm{CHCl}_{3}\right.$ :hexanes). The final product was obtained as a colorless oil $(0.0814 \mathrm{~g}, 16.4 \%$ yield $) .{ }^{1} \mathrm{H} \mathrm{NMR}\left(\mathrm{CD}_{3} \mathrm{Cl}\right.$ $400 \mathrm{MHz}) \delta 7.79(\mathrm{dd}, \mathrm{J}=8.4,7.3 \mathrm{~Hz}, 1 \mathrm{H}), 7.71(\mathrm{~d}, \mathrm{~J}=3.7 \mathrm{~Hz}, 2 \mathrm{H}), 7.65(\mathrm{~d}, \mathrm{~J}=7.7 \mathrm{~Hz}$, 2H), $7.52(\mathrm{~d}, \mathrm{~J}=5.1 \mathrm{~Hz}, 2 \mathrm{H}), 7.16(\mathrm{dd}, \mathrm{J}=5.1,3.7 \mathrm{~Hz}, 2 \mathrm{H}) .{ }^{13} \mathrm{C} \mathrm{NMR}(100 \mathrm{MHz}$, $\left.\mathrm{CD}_{3} \mathrm{CN}\right) \delta 151.84,137.83,128.17,128.07,125.03,116.79$. FTMS + p ESI calcd for $\mathrm{C}_{13} \mathrm{H}_{9} \mathrm{NS}_{2}, 244.02492$; found, 244.02667.

\subsubsection{Synthesis of 2-(dibenzo[b,d]thiophen-4-yl)pyridine (BT1)}

Degassed dimethoxyethane $(30 \mathrm{~mL})$ and water $(10 \mathrm{~mL})$ were transferred to a flask, purged of air, containing dibenzothiophen-4-ylboronic acid (0.4710 g, $2.06 \mathrm{mmol})$, 2-bromopyridine $(0.197 \mathrm{~mL}, \quad 2.06 \mathrm{mmol}), \quad \mathrm{Pd}\left(\mathrm{PPh}_{3}\right)_{4} \quad(0.2831 \mathrm{~g}, \quad 0.245 \mathrm{mmol})$ and potassium carbonate $(0.4158 \mathrm{~g}, 3.01 \mathrm{mmol})$ under flowing $\mathrm{N}_{2}$. This solution was heated under reflux for approximately 6 hours before it was cooled to room temperature. Additional water was added $(\sim 10 \mathrm{~mL})$, the organic layer was then separated. The aqueous layer was extracted with $\mathrm{CHCl}_{3}(3 \times 50 \mathrm{~mL})$, organic layers were combined and dried 
over $\mathrm{Na}_{2} \mathrm{SO}_{4}$, filtered, and the solvent was evaporated in vacuo. The crude residue was then purified via column chromatography over silica gel using a 2:1 eluent mixture of $\mathrm{CH}_{2} \mathrm{Cl}_{2}$ :hexanes, affording the pure product as a white solid $(0.4437 \mathrm{~g}, 82.3 \%$ yield $)$. ${ }^{1} \mathrm{H}$ NMR $\left(600 \mathrm{MHz}, \mathrm{CD}_{3} \mathrm{CN}\right) \delta 8.87(\mathrm{~d}, J=4.8 \mathrm{~Hz}, 1 \mathrm{H}), 8.42(\mathrm{~d}, J=7.8 \mathrm{~Hz}, 1 \mathrm{H}), 8.38-$ $8.31(\mathrm{~m}, 1 \mathrm{H}), 8.20(\mathrm{~d}, J=7.6 \mathrm{~Hz}, 1 \mathrm{H}), 8.17(\mathrm{~d}, J=8.1 \mathrm{~Hz}, 1 \mathrm{H}), 8.05-8.01(\mathrm{~m}, 1 \mathrm{H}), 8.01$ $-7.96(\mathrm{~m}, 1 \mathrm{H}), 7.71(\mathrm{t}, J=7.7 \mathrm{~Hz}, 1 \mathrm{H}), 7.60-7.52(\mathrm{~m}, 2 \mathrm{H}), 7.44$ (ddd, $J=7.4,4.8,1.0$ $\mathrm{Hz}, 1 \mathrm{H})$. FTMS + p ESI calcd for $\mathrm{C}_{17} \mathrm{H}_{12} \mathrm{NS}, 262.06905$; found, 262.06814.

\subsubsection{Synthesis of 2-(dibenzo[b,d]thiophen-4-yl)-1H-imidazole (BT2)}

A flask containing dibenzo[b,d]thiophen-4-ylboronic acid $(0.2747 \mathrm{~g}, 1.20 \mathrm{mmol})$, 2-bromo-1H-imidazole (0.1884 g, $1.28 \mathrm{mmol}), \mathrm{Pd}\left(\mathrm{PPh}_{3}\right)_{4}(0.0540 \mathrm{~g}, 0.047 \mathrm{mmol})$, and caesium carbonate $(0.4398 \mathrm{~g}, 1.35 \mathrm{mmol})$ was purged of air and filled with $\mathrm{N}_{2}$ gas. Degassed toluene $(20 \mathrm{~mL})$ and water $(10 \mathrm{~mL})$ were then added to the flask containing the solid reagents and heated under reflux for $18 \mathrm{hr}$. Once cooled to room temperature the organic and aqueous layers were separated and the aqueous layer was extracted with EtOAc $(3 \times 50 \mathrm{~mL})$. The combined organic layers were dried over $\mathrm{Na}_{2} \mathrm{SO}_{4}$, filtered, and then the solvent was evaporated in vacuo. The crude product was purified via column chromatography over silica gel starting with hexanes as the eluent, then 50:50 hexanes:EtOAc, and finally EtOAc. This afforded a white solid which was dissolved in EtOAc $(50 \mathrm{~mL})$ and subsequently washed with saturated sodium bicarbonate to remove residual 2-bromo[1H]-imidazole. The organic layer was dried over $\mathrm{Na}_{2} \mathrm{SO}_{4}$, filtered and solvent was evaporated to afford the pure product as a white solid $(0.2671 \mathrm{~g}$

88.6\% yield). ${ }^{1} \mathrm{H}$ NMR $\left(600 \mathrm{MHz}, \mathrm{CD}_{3} \mathrm{CN}\right) \delta 10.82(\mathrm{~s}, 1 \mathrm{H}), 8.22(\mathrm{dd}, J=6.3,1.5 \mathrm{~Hz}$, 
1H), $7.93(\mathrm{~d}, J=6.9 \mathrm{~Hz}, 1 \mathrm{H}), 7.82(\mathrm{~d}, J=7.5 \mathrm{~Hz}, 1 \mathrm{H}), 7.55(\mathrm{t}, J=7.7 \mathrm{~Hz}, 1 \mathrm{H}), 7.45$ (ddd, $J=6.9,5.2,1.6 \mathrm{~Hz}, 1 \mathrm{H}$ ). FTMS - p ESI calcd for $\mathrm{C}_{15} \mathrm{H}_{10} \mathrm{~N}_{2} \mathrm{~S}, 249.04864$; found, 249.04947.

\subsubsection{Synthesis of 2-(dibenzo[b,d] thiophen-4-yl)thiazole (BT3)}

A flask containing dibenzo[b,d]thiophen-4-ylboronic acid $(0.2462 \mathrm{~g}, 1.07 \mathrm{mmol})$, 2-bromothiazole (0.107 mL, $1.18 \mathrm{mmol}), \mathrm{Pd}\left(\mathrm{PPh}_{3}\right)_{4}(0.0584 \mathrm{~g}, 0.047 \mathrm{mmol})$, and caesium carbonate $(0.3610 \mathrm{~g}, 1.11 \mathrm{mmol})$ was purged of air and filled with $\mathrm{N}_{2}$ and degassed solvents (toluene $-20 \mathrm{~mL} ; \mathrm{H}_{2} \mathrm{O}-10 \mathrm{~mL}$ ) were subsequently added. The reaction was heated under reflux and the solution was stirred for $18 \mathrm{hr}$. Once the solution had been cooled to room temperature the layers were separated, and the aqueous layer was extracted with EtOAc $(3 \times 50 \mathrm{~mL})$. The combined organic layers were dried over $\mathrm{Na}_{2} \mathrm{SO}_{4}$, filtered and the solvent was evaporated in vacuo. The crude product was then purified via column chromatography over silica gel using 95:5 hexanes:EtOAc as the eluent mixture to afford the pure product as a light yellow solid (0.1576 g $54.6 \%$ yield).

${ }^{1} \mathrm{H}$ NMR $\left(600 \mathrm{MHz}, \mathrm{CD}_{3} \mathrm{CN}\right) \delta 8.43(\mathrm{dd}, J=7.8,0.9 \mathrm{~Hz}, 1 \mathrm{H}), 8.38-8.34(\mathrm{~m}, 1 \mathrm{H}), 8.15$ $(\mathrm{dd}, J=7.5,0.9 \mathrm{~Hz}, 1 \mathrm{H}), 8.08(\mathrm{~d}, J=3.2 \mathrm{~Hz}, 1 \mathrm{H}), 8.07(\mathrm{dd}, J=6.4,2.1 \mathrm{~Hz}, 1 \mathrm{H}), 7.69(\mathrm{~d}$, $J=7.7 \mathrm{~Hz}, 1 \mathrm{H}), 7.67(\mathrm{~d}, J=3.3 \mathrm{~Hz}, 1 \mathrm{H}), 7.62-7.55(\mathrm{~m}, 2 \mathrm{H})$. FTMS + p ESI calcd for $\mathrm{C}_{15} \mathrm{H}_{10} \mathrm{NS}_{2}, 268.02547$; found, 268.02460.

\subsubsection{Synthesis of 6,6'-thiophene-2,5-diyl)bis(pyridine-3-ol) (P1)}


To a flask filled with nitrogen gas, thiophene-2,5-diyldiboronic acid $(0.431 \mathrm{~g}, 2.51$ mmol), 6-bromopyridin-3-ol (0.914 g, $5.25 \mathrm{mmol})$, potassium fluoride $(0.3363 \mathrm{~g}, 5.78$ mmol), and $\quad \mathrm{Pd}\left(\mathrm{PPh}_{3}\right)_{4} \quad\left(\begin{array}{llllll}0.102 & \mathrm{~g}, & 0.08 & \mathrm{mmol}) & \text { were } & \text { added. Degassed }\end{array}\right.$ dimethoxyethane $(13.3 \mathrm{~mL})$ and $\mathrm{H}_{2} \mathrm{O}(6.67 \mathrm{~mL})$ were added to the flask and the solution was heated under reflux for 4 hours. The reaction was cooled to room temperature and 20 $\mathrm{mL}$ of $\mathrm{H}_{2} \mathrm{O}$ was added. The crude product was extracted with dichloromethane $(3 \times 50$ $\mathrm{mL}$ ). The organic layers were combined and dried over $\mathrm{Na}_{2} \mathrm{SO}_{4}$, then filtered and the solvent was evaporated in vacuo. The crude product was purified by column chromatography using $90: 10 \mathrm{CH}_{2} \mathrm{Cl}_{2}: \mathrm{MeOH}$ as the eluent. The pure product was obtained as a yellow solid $(0.211 \mathrm{~g}, \quad 31.1 \%$ yield $) .{ }^{1} \mathrm{H} \quad \mathrm{NMR} \quad(400 \mathrm{MHz}, \mathrm{MeOD})$ $\delta 8.09(\mathrm{~d}, \mathrm{~J}=2.8 \mathrm{~Hz}, 1 \mathrm{H}), 7.69(\mathrm{~d}, \mathrm{~J}=8.7 \mathrm{~Hz}, 1 \mathrm{H}), 7.48(\mathrm{~s}, 1 \mathrm{H}), 7.25(\mathrm{dd}, \mathrm{J}=8.7,2.9 \mathrm{~Hz}$, $1 \mathrm{H}) ;{ }^{13} \mathrm{C}$ NMR (100 MHz, MeOD) $\delta 154.66,145.68,145.57,138.10,125.32,124.78$, 121.40, FTMS + p ESI Calcd for $\mathrm{C}_{14} \mathrm{H}_{11} \mathrm{~N}_{2} \mathrm{O}_{2} \mathrm{~S}, 271.05412$; found, 271.05396, IR (KBr): $749,918,1039,1375,1444,2943,3002,3164,3620 \mathrm{~cm}^{-1}$.

\subsubsection{Synthesis of 2,5-bis(5-((2,5,8,11,14,17,20-heptaoxadocosan-22-yl)oxy)pyridine-2- yl)thiophene (P2)}

To a flask filled with nitrogen gas $\mathbf{P 1}(0.262, \mathrm{~g}, 0.97 \mathrm{mmol})$ and cesium carbonate (2.321 g, $5.15 \mathrm{mmol})$ were added. Dry DMF ( 100 $\mathrm{mL})$ was added to the flask and the solution was heated to $75{ }^{\circ} \mathrm{C}$ and left stirring for 1 hour. A $0.17 \mathrm{M}$ solution of 22 -iodo2,5,8,11,14,17,20-heptaoxadocosane (15 mL) was added to the reaction solution over 15 minutes, and stirred for 6 hours resulting in an orange solution. A second addition of the $0.17 \mathrm{M}$ solution of 22-iodo-2,5,8,11,14,17,20-heptaoxadocosane $(15 \mathrm{~mL})$ was performed 
over 15 minutes and stirred for 8 hours. Solvent was evaporated in vacuo and the crude product was dissolved in $\mathrm{CH}_{2} \mathrm{Cl}_{2}$ and washed with $\mathrm{H}_{2} \mathrm{O}(3 \times 20 \mathrm{~mL})$. The aqueous phase was then extracted with $\mathrm{CH}_{2} \mathrm{Cl}_{2}(3 \times 50 \mathrm{~mL})$. The combined organic layers were then washed with brine $(2 \times 50 \mathrm{~mL})$ and the aqueous phases were subsequently extracted once more with $\mathrm{CH}_{2} \mathrm{Cl}_{2}(2 \times 50 \mathrm{~mL})$. Combined organic phases were dried over $\mathrm{Na}_{2} \mathrm{SO}_{4}$, filtered and solvent was evaporated in vacuo to afford a red oil. The product was purified via column chromatography using EtOAc:TEA (99:1) followed by EtOAc:MeOH:TEA (99:9:1) affording the pure product as an orange oil (0.6212 g, 70\% yield). ${ }^{1} \mathrm{H}$ NMR (400 MHz, MeOD) $\delta 8.25(\mathrm{~d}, J=2.6 \mathrm{~Hz}, 1 \mathrm{H}), 7.80(\mathrm{~d}, J=8.8 \mathrm{~Hz}, 1 \mathrm{H}), 7.55(\mathrm{~s}, 1 \mathrm{H}), 7.47$ (dd, $J=8.8,2.9 \mathrm{~Hz}, 1 \mathrm{H}), 4.30-4.25(\mathrm{t}, J=5.3 \mathrm{~Hz}, 2 \mathrm{H}), 3.90(\mathrm{t}, J=5.3 \mathrm{~Hz}, 2 \mathrm{H}), 3.33(\mathrm{~m}$, $29 \mathrm{H}) ;{ }^{13} \mathrm{C}$ NMR $(100 \mathrm{MHz}, \mathrm{MeOD}) \delta 155.85,146.74,145.94,138.39,125.86,123.73$, $121.02,73.30,72.77,72.71,71.65,71.36,71.29,71.24,71.11,71.09,71.05,70.97,70.66$ 69.34, 61.89, 59.13; FTMS + p ESI Calcd for $\mathrm{C}_{44} \mathrm{H}_{70} \mathrm{~N}_{2} \mathrm{O}_{16} \mathrm{~S}$, 914.44460; found, 915.45687, IR (KBr): 628, 8443, 918, 949, 1039, 1105, 1250, 1271, 1350, 1375, 1452, $1567,2881 \mathrm{~cm}^{-1}$.

\subsubsection{Synthesis of 6,6'-(thiophene-2,5-diyl)bis(pyridine-3-amine) (P3)}

To a flask filled with $\mathrm{N}_{2}$ gas thiophene-2,5-diyldiboronic acid (0.419 $\mathrm{g}$, $2.44 \mathrm{mmol})$, 6-bromopyridin-3-amine $(0.898 \mathrm{~g}, 5.19 \mathrm{mmol})$, potassium fluoride $(0.274 \mathrm{~g}$, $4.72 \mathrm{mmol})$, and $\quad \mathrm{Pd}\left(\mathrm{PPh}_{3}\right)_{4} \quad(0.094 \mathrm{~g}, \quad 0.08 \mathrm{mmol})$ were added. Degassed dimethoxyethane $(13.3 \mathrm{~mL})$ and $\mathrm{H}_{2} \mathrm{O}(6.67 \mathrm{~mL})$ were added to the flask and the solution was heated under reflux for 4 hours resulting in a dark red solution. The solution was cooled to room temperature and the crude product was extracted with ethyl acetate 
$(3 \times 50 \mathrm{~mL})$. The organic layers were combined and dried over $\mathrm{Na}_{2} \mathrm{SO}_{4}$ and then filtered. The solvent was evaporated in vacuo to afford a red oil. The crude product was purified via column chromatography using 99:1 EtOAc:TEA as the eluent. The pure product was obtained as a red solid $\left(0.253 \mathrm{~g}, 38.7 \%\right.$ yield). ${ }^{1} \mathrm{H}$ NMR $(400 \mathrm{MHz}, \mathrm{MeOD}) \delta 7.84(\mathrm{~d}, J=$ $2.7 \mathrm{~Hz}, 1 \mathrm{H}), 7.43(\mathrm{~d}, J=8.6 \mathrm{~Hz}, 1 \mathrm{H}), 7.27(\mathrm{~s}, 1 \mathrm{H}), 6.99(\mathrm{dd}, J=8.6,2.8 \mathrm{~Hz}, 1 \mathrm{H})$; ${ }^{13} \mathrm{C}$ NMR (100 MHz, MeOD) $\delta 146.05,145.22,145.13,136.68,124.43,123.43,121.33$; FTMS + p ESI Calcd for $\mathrm{C}_{14} \mathrm{H}_{13} \mathrm{~N}_{4} \mathrm{~S}, 269.08609$; found, 269.08579; IR (KBr): 748, 918, $1039,1375,1444,2943,3002,3163,3622 \mathrm{~cm}^{-1}$.

\subsubsection{Synthesis of 2,2'-(3,4-dimethoxythiophene-2,5-diyl)dipyridine (T1)}

To the flame-dried reaction flask, 2,5-dibromo-3,4-dimethoxythiophene (1.2660 g, $4.19 \mathrm{mmol}), \quad$ 2-(trimethylstannyl)-pyridine $\quad(2.3656 \mathrm{~g}, \quad 9.77 \mathrm{mmol}), \quad \mathrm{PdCl}_{2}\left(\mathrm{PPh}_{3}\right)_{2}$ $(0.0415 \mathrm{~g}, 0.059 \mathrm{mmol})$ and dry degassed toluene $(\sim 20 \mathrm{~mL})$ was added under $\mathrm{N}_{2}$ gas. The solution was heated to $110{ }^{\circ} \mathrm{C}$ and left stirring overnight. After cooling to room temperature the organic layer was washed with $\mathrm{H}_{2} \mathrm{O}(3 \times 20 \mathrm{~mL})$ and the organic layer was dried over $\mathrm{Na}_{2} \mathrm{SO}_{4}$. The organic solution was then filtered and evaporated in vacuo to afford the crude product. The crude product was then purified via column chromatography over silica gel using 95:5 hexanes:EtOAc as an eluent affording the pure product as a yellow-solid $(0.3495 \mathrm{~g}, 27.9 \%$ yield $) .{ }^{1} \mathrm{H}$ NMR $\left(400 \mathrm{MHz}, \mathrm{CDCl}_{3}\right) \delta 8.57$ $(\mathrm{d}, J=4.8 \mathrm{~Hz}, 1 \mathrm{H}), 8.03(\mathrm{~d}, J=8.1 \mathrm{~Hz}, 1 \mathrm{H}), 7.69(\mathrm{t}, J=7.8 \mathrm{~Hz}, 1 \mathrm{H}), 7.17-7.09(\mathrm{~m}$, 1H), $3.98(\mathrm{~s}, 3 \mathrm{H}) ;{ }^{13} \mathrm{C} \mathrm{NMR}\left(100 \mathrm{MHz}, \mathrm{CDCl}_{3}\right) \delta 151.53,149.49,148.68,136.60$, 127.86, 121.96, 120.86, 60.40; FTMS + p ESI Calcd for $\mathrm{C}_{16} \mathrm{H}_{15} \mathrm{~N}_{2} \mathrm{O}_{2} \mathrm{~S}, 299.08542$; found, 299.08584; IR (KBr): 749, 918, 1375, 1443, 2943, 3002, $3163 \mathrm{~cm}^{-1}$. 


\subsubsection{Synthesis of 2,5-di(pyridin-2-yl)thiophene-3,4-diol (T2)}

Pyridine hydrochloride was generated by addition of concentrated $\mathrm{HCl}(3.52 \mathrm{~mL}$, $12.78 \mathrm{M})$ to pyridine $(3.17 \mathrm{~mL}, 39.3 \mathrm{mmol})$ in a reaction flask. Water was then evaporated under high vacuum at $150{ }^{\circ} \mathrm{C}$, resulting in molten pyridine hydrochloride. Solid T1 (0.1397 g, $0.468 \mathrm{mmol})$ was added to the reaction flask after which the solution darkened to a red color. After 10 minutes of stirring the solution was cooled to $80{ }^{\circ} \mathrm{C}$. $\mathrm{H}_{2} \mathrm{O}\left(90{ }^{\circ} \mathrm{C}\right)$ was added to the reaction flask and subsequently poured over lukewarm $\mathrm{H}_{2} \mathrm{O}$ in a beaker and allowed to cool to room temperature. The aqueous solution was neutralized to $\mathrm{pH} 7$ with saturated $\mathrm{NaHCO}_{3}$ solution after which the product was extracted using EtOAc $(3 \times 50 \mathrm{~mL})$. The combined organic layers were washed again with saturated $\mathrm{NaHCO}_{3}$ and then brine after which they were dried over $\mathrm{Na}_{2} \mathrm{SO}_{4}$, filtered and the solvent was evaporated in vacuo. The crude product was purified via column chromatography over silica gel using 50:50 hexanes:EtOAc as the eluent, affording the pure product as an orange solid $\left(0.087 \mathrm{~g}, 68.7 \%\right.$ yield). ${ }^{1} \mathrm{H}$ NMR $\left(400 \mathrm{MHz}, \mathrm{CDCl}_{3}\right) \delta$ $12.68(\mathrm{~s}, 1 \mathrm{H}), 8.47(\mathrm{~d}, J=4.9 \mathrm{~Hz}, 1 \mathrm{H}), 7.73(\mathrm{t}, J=7.8 \mathrm{~Hz}, 1 \mathrm{H}), 7.30(\mathrm{~d}, J=8.1 \mathrm{~Hz}, 1 \mathrm{H})$,

$7.17-7.08(\mathrm{~m}, 1 \mathrm{H}) ;{ }^{13} \mathrm{C}$ NMR $\left(100 \mathrm{MHz}, \mathrm{CDCl}_{3}\right) \delta 154.65,149.31,147.04,137.54$, 120.68, 118.30, 109.58; FTMS + p ESI Calcd for $\mathrm{C}_{14} \mathrm{H}_{11} \mathrm{~N}_{2} \mathrm{O}_{2} \mathrm{~S}$, 271.05412; found, 271.05410; IR (KBr): 749, 918, 1039, 1375, 1421, 1444, 2943, 3003, 3163, $3583 \mathrm{~cm}^{-1}$.

\subsubsection{Synthesis of 2,2'-(3,4-bis((2,5,8,11,14,17,20-heptaoxadocosan-22-} yl)oxy)thiophene-2,5-diyl)dipyridine (T3) 
To a flask purged of air and filled with $\mathrm{N}_{2}, \mathbf{T 2}(0.0918 \mathrm{~g}, 0.339 \mathrm{mmol})$ and cesium carbonate $(0.7010 \mathrm{~g}, 2.15 \mathrm{mmol})$ was added and dissolved in dry degassed DMF upon which the solution turned red. The reaction flask was then heated to $75{ }^{\circ} \mathrm{C}$ and stirred vigorously for 1 hour, after which $0.4 \mathrm{M}$ iodoheptaethyleneglycol monomethyl ether solution $(2.5 \mathrm{~mL})$ in DMF was added over 10 minutes and left stirring overnight at $75^{\circ} \mathrm{C}$. The next day a second addition of $0.4 \mathrm{M}$ iodoheptaethyleneglycol monomethyl ether (2.5 mL) was added over 10 minutes at $75^{\circ} \mathrm{C}$ and the reaction solution was left stirring for an additional $18 \mathrm{hr}$. The flask was cooled to room temperature and the solvent was evaporated under vacuum. The dark residue was dissolved in $\mathrm{CH}_{2} \mathrm{Cl}_{2}(50 \mathrm{~mL})$ and $\mathrm{H}_{2} \mathrm{O}$ $(50 \mathrm{~mL})$ and the organic layer was removed. Solid $\mathrm{NaCl}(7.9808 \mathrm{~g})$ was added to the aqueous layer and the product was extracted with $\mathrm{CH}_{2} \mathrm{Cl}_{2}(4 \times 25 \mathrm{~mL})$. The combined organic layers were washed with brine and subsequently dried over $\mathrm{Na}_{2} \mathrm{SO}_{4}$, filtered and the solvent was evaporated in vacuo. The crude product was purified via column chromatography over silica gel using 99:1 to $95: 5 \mathrm{CH}_{2} \mathrm{Cl}_{2}: \mathrm{MeOH}$ affording a brown oil. This oil was then dissolved in EtOAc and washed with $1 \mathrm{M} \mathrm{HCl}(3 \times 20 \mathrm{~mL})$ after which the organic layer was discarded. The aqueous layer was subsequently neutralized with solid $\mathrm{NaHCO}_{3}, \mathrm{NaCl}(\sim 5 \mathrm{~g})$ was added, and the product was then extracted with EtOAc $(3 \times 50 \mathrm{~mL})$. The combined organic layers were dried over $\mathrm{Na}_{2} \mathrm{SO}_{4}$, after which the solvent was evaporated in vacuo to obtain the pure product as a yellow oil $(0.2521 \mathrm{~g}$, 81.1\% yield). ${ }^{1} \mathrm{H}$ NMR $\left(400 \mathrm{MHz}, \mathrm{CDCl}_{3}\right) \delta 8.55(\mathrm{~d}, J=4.0 \mathrm{~Hz}, 1 \mathrm{H}), 8.21(\mathrm{~d}, J=8.0 \mathrm{~Hz}$, 1H), $7.69(\mathrm{t}, J=7.8 \mathrm{~Hz}, 1 \mathrm{H}), 7.14-7.09(\mathrm{~m}, 1 \mathrm{H}), 4.37-4.32(\mathrm{~m}, 2 \mathrm{H}), 4.23-4.20(\mathrm{~m}$, $1 \mathrm{H}), 3.85-3.58(\mathrm{~m}, 102 \mathrm{H}), 3.58-3.51(\mathrm{~m}, 8 \mathrm{H}), 3.38(\mathrm{~s}, 7 \mathrm{H}) ;{ }^{13} \mathrm{C} \mathrm{NMR}(100 \mathrm{MHz}$, 
$\left.\mathrm{CDCl}_{3}\right) \delta 149.19,139.95,136.49,121.87,121.28,113.95,111.54,72.60,71.94,71.37$, 70.58, 70.31, 70.16, 61.74, 59.05, 42.73; FTMS + p ESI Calcd for $\mathrm{C}_{44} \mathrm{H}_{70} \mathrm{~N}_{2} \mathrm{O}_{16} \mathrm{~S}$, 915.45243; found, 915.45687; IR (KBr): 918, 1039, 1375, 1444, 2943, 3003, $3163 \mathrm{~cm}^{-1}$.

\subsubsection{Synthesis of 2,2'-(3,4-dinitrothiophene-2,5-diyl)dipyridine}

To a flame-dried flask purged of air and filled with $\mathrm{N}_{2}$, 2,5-dibromo-3,4dinitrothiophene $\quad(0.3353 \mathrm{~g}, \quad 1.01 \mathrm{mmol}), \quad$ 2-(trimethylstannyl)pyridine $\quad(0.4982 \mathrm{~g}$, $2.06 \mathrm{mmol})$, and $\mathrm{Pd}\left(\mathrm{PPh}_{3}\right)_{4}(0.0402 \mathrm{~g}, 0.035 \mathrm{mmol})$ was added. Dry, degassed toluene $(20 \mathrm{~mL})$ was then added to the flask and the solution was heated to reflux and left stirring overnight. The solvent was evaporated after cooling to room temperature and the crude solid was purified via column chromatography over silica gel with $\mathrm{CH}_{2} \mathrm{Cl}_{2}$ as the eluent. This afforded the pure product as a yellow-green solid (0.1028 g $30.9 \%$ yield). ${ }^{1} \mathrm{H}$ NMR $\left(400 \mathrm{MHz}, \mathrm{CDCl}_{3}\right) \delta 8.68(\mathrm{~d}, J=4.8 \mathrm{~Hz}, 1 \mathrm{H}), 7.82(\mathrm{td}, J=7.8,1.6 \mathrm{~Hz}, 1 \mathrm{H})$, $7.67(\mathrm{~d}, J=8.0 \mathrm{~Hz}, 1 \mathrm{H}), 7.40(\mathrm{dd}, J=7.5,4.9 \mathrm{~Hz}, 1 \mathrm{H}) ; \mathrm{IR}(\mathrm{KBr}): 748,896,992,1090$, 1158, 1222, 1274, 1331, 1362, 1421, 1533, 1586, 1712, 2855, 2928 2987, $3054 \mathrm{~cm}^{-1}$.

\subsubsection{Synthesis of 2,5-di(pyridin-2-yl)thiophene-3,4-diamine (T4)}

A solution of 2,2'-(3,4-dinitrothiophene-2,5-diyl)dipyridine (0.1028 g $0.313 \mathrm{mmol})$ was prepared in $\mathrm{CH}_{2} \mathrm{Cl}_{2}(2.5 \mathrm{~mL})$ and $\mathrm{EtOH}(2.5 \mathrm{~mL})$ in a reaction flask. Tin(II) chloride was dissolved in $\mathrm{EtOH}(4 \mathrm{~mL})$ and $\mathrm{HCl}(2.35 \mathrm{~mL}, 1.35 \mathrm{M})$. These solutions were degassed by bubbling $\mathrm{N}_{2}$ gas, after which the tin(II) chloride solution was added dropwise to the reaction flask containing the $\mathrm{CH}_{2} \mathrm{Cl}_{2}$ : $\mathrm{EtOH}$ solution under flowing $\mathrm{N}_{2}$ resulting in a red solution. The reaction flask was then heated to $30{ }^{\circ} \mathrm{C}$ and stirred for 
3 days before addition of $25 \% \mathrm{NaOH}$ to quench the reaction after which the solution was cooled to room temperature. The layers were separated and the organic layer was removed. The aqueous layer was diluted in a $0.5 \mathrm{M}$ EDTA solution $(20 \mathrm{~mL})$ and then extracted with $\mathrm{CH}_{2} \mathrm{Cl}_{2}(3 \times 30 \mathrm{~mL})$. The combined organic layers were washed with the $0.5 \mathrm{M}$ EDTA solution $(2 \times 20 \mathrm{~mL})$ and then brine. The organic layer was then dried over $\mathrm{Na}_{2} \mathrm{SO}_{4}$, filtered and the solvent was evaporated in vacuo to afford the pure product as a red-solid (0.0845 g 99\% yield). ${ }^{1} \mathrm{H}$ NMR $\left(400 \mathrm{MHz}, \mathrm{CDCl}_{3}\right) \delta 8.52(\mathrm{~d}, J=4.1 \mathrm{~Hz}, 1 \mathrm{H})$, $7.63(\operatorname{td}, J=7.9,1.8 \mathrm{~Hz}, 1 \mathrm{H}), 7.37(\mathrm{~d}, J=8.1 \mathrm{~Hz}, 1 \mathrm{H}), 7.02(\mathrm{ddd}, J=7.4,4.9,0.9 \mathrm{~Hz}$, 1H), $5.72(\mathrm{~s}, 2 \mathrm{H}) ;{ }^{13} \mathrm{C} \mathrm{NMR}\left(100 \mathrm{MHz}, \mathrm{CDCl}_{3}\right) \delta 155.18,148.21,139.79,136.43$, 119.54, 111.17; FTMS + p ESI Calcd for $\mathrm{C}_{14} \mathrm{H}_{13} \mathrm{~N}_{4} \mathrm{~S}$, 269.08609; found, 269.08789; IR (KBr): 707, 918, 1039, 1375, 1448, 2943, 3003, 3163, $3583 \mathrm{~cm}^{-1}$.

\subsection{Measurement of Quantum Yields}

Quantum yields were determined for each ligand solution at the same concentrations used for absorption and emission titrations in $\mathrm{CH}_{3} \mathrm{CN}$ with an integrating sphere. To determine the amount of light produced an emission scan was performed over the excitation wavelength of the sensor first with a blank sample, both with and without an optical density filter, and then with a solution of the sensor. The optical density filters were varied, depending on the amount of light absorbed by the sensor ( $0.5 \mathrm{OD}, 0.7 \mathrm{OD}$, or $1.0 \mathrm{OD}$ ). After this the amount of light produced was measured by obtaining a corrected emission spectrum of the sensor. Quantum yields were then calculated by using the Quantum yield calculator of the FelixGX software. 


\subsection{General Procedure for Job's Plots}

Solutions of both metal perchlorate salt and sensor were prepared at $0.001 \mathrm{M}$ concentration in $\mathrm{CH}_{3} \mathrm{CN}$ using volumetric flasks. Several different solutions of varying mole fractions of the sensor and mercury(II) were prepared from these stock solutions in a $10 \mathrm{~mL}$ volumetric flask. The total concentration of both mercury(II) and sensor in solution was $2 \times 10^{-5} \mathrm{M}$. Each solution was allowed to stir for approximately 5 min each to ensure they were at equilibrium, after which absorption spectrum were obtained for each.

\subsection{General Procedure for Absorption and Emission Titrations}

Ligand solutions were prepared in $\mathrm{CH}_{3} \mathrm{CN}$ at varying concentrations (L1-L3: $1 \times 10^{-5} \mathrm{M}$; BT1-BT3: $3 \times 10^{-5} \mathrm{M}$; P1-P3: $1 \times 10^{-5} \mathrm{M}$; T1,T2, \& T4: $1 \times 10^{-5} \mathrm{M}$; T3: $2 \times 10^{-5} \mathrm{M}$ ). The metal salts solutions were prepared fresh the day of the experiment at either $0.02 \mathrm{M}$ or $0.2 \mathrm{M}$ concentrations in a solution of the corresponding ligand used. When over 100 equivalents were needed $0.2 \mathrm{M}$ solutions of metal salt were prepared. After an initial spectrum of the ligand was obtained, increasing molar equivalents of the metal salts were added to the solution, via micropipette, until no change in the spectra was observed.

\subsection{General Procedure for ${ }^{1} H$ NMR Experiments}

${ }^{1} \mathrm{H}$ NMR titrations were performed by preparing $4 \times 10^{-3} \mathrm{M}$ ligand solutions in $\mathrm{CD}_{3} \mathrm{CN}$. An initial ${ }^{1} \mathrm{H}$ NMR spectrum was obtained of the ligand prior to addition of a 
metal salt. Increasing equivalents of the metal salt were added to the solution until no change in the spectrum was observed.

\subsection{General Procedure for Competition Experiments}

Solutions of silver(I), cadmium(II), cobalt(II), copper(II), iron(II), iron(III), magnesium(II), manganese(II), sodium(I), nickel(II), lead(II), and zinc(II) perchlorate salts $(0.02 \mathrm{M})$ and a solution of sensor at concentrations identical to the titration were prepared in $\mathrm{CH}_{3} \mathrm{CN}$. An absorption or emission spectrum was obtained for the ligand solution. 5 eq of metal perchlorate was added to a $10 \mathrm{~mL}$ aliquot the ligand solution. After approximately 5 minutes of stirring an emission spectrum was obtained. After which 5 eq of $\mathrm{Hg}\left(\mathrm{ClO}_{4}\right)_{2}$ in $\mathrm{CH}_{3} \mathrm{CN}$ was added to each solution and the solution was allowed to stir for 5 minutes before another emission spectrum was obtained.

\subsection{Computational Chemistry}

DFT calculations were run using Gaussian $09^{80}$ with the B3LYP ${ }^{81,82}$ level of theory and $6-311+\mathrm{G}(\mathrm{d})$ basis set for all atoms. Optimizations and TD-DFT calculations were performed for all sensors except BT1-BT3. The UV-vis spectrum generated from the TD-DFT data with the full width at half max for Gaussian functions at $0.3 \mathrm{eV}$. 


\title{
Chapter 3. Thiophene-Based Ratiometric Sensors in Organic Solvent
}

\author{
This work has been published in Journal of Coordination Chemistry
}

Shigemoto, A. K., Virca, C. N., Underwood, S. J., Shetterly, L. R., and Mccormick, T. M. (2016) Thiophene-based fluorescent mercury-sensors. J. Coord. Chem. 8972, 1-9.

\subsection{Introduction}

In this chapter the first generation of sensors that were developed for this project will be discussed. These sensors included both pyridine and thiophene moieties coupled together. These sensors 2-(2'-thienyl)-pyridine (L1), 2,5-bis(2-pyridyl)thiophene (L2), and 2,6-bis(2-thienyl)pyridine (L3) (Figure 7) were designed as both bidentate and tridentate ligands incorporating a different ratio of pyridine and thiophene rings $(1: 1,2: 1$, and $1: 2) .{ }^{83}$ By varying the ratio of thiophene and pyridine rings the ability of thiophene as a chelating group was investigated. Comparison of the magnitude of $K_{\mathrm{a}}$ values of $\mathbf{L} \mathbf{2}$ and L3 will provide a method of identifying which ring is the more ideal chelating group. These ligands were designed to promote sulfur-coordination of the thiophene, by implementing chelating groups at the 2,5-position of the ring. Since chelation of the pyridyl nitrogen to the thienyl carbon of the 3,4-position is possible in L1-L3 ${ }^{1} \mathrm{H}$ NMR titrations were performed to elucidate the coordination mode of the ligands. Furthermore determination of the $K_{\mathrm{a}}$ for each ligand revealed the binding stoichiometry as well. Herein we will review the supramolecular properties of these mercury(II) sensors, and summarize the photophysical properties of the free ligands and formed metal complexes. 


\subsection{Results}

\subsubsection{Ligand Synthesis and Structure}

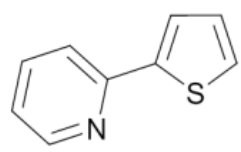

L1

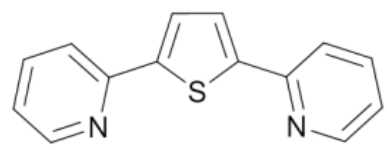

L2

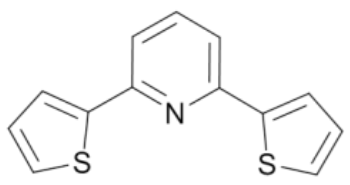

L3

Figure 7: Structural depiction of L1-L3.

L1 from this study was purchased from TCI chemicals and used after purification by recrystallization in hexanes. $\mathbf{L 2}$ and $\mathbf{L 3}$ were synthesized via Suzuki coupling reactions following modified literature procedures (Scheme 4) ${ }^{84}$ In the case of L2 dioxane, instead of toluene, as the solvent afforded the title product in greater yield (37.1\% yield). Several synthetic routes were attempted for the synthesis of $\mathbf{L} \mathbf{3}$ before the desired product was obtained. The first attempt to synthesize $\mathbf{L} \mathbf{3}$ attempted to couple the thienyl and pyridyl moieties using pyridine-2,6-diyldiboronic acid and 2-bromothiophene affording no product. Following this the 2-thienylboronic acid and 2,6-dibromopyridine were used however no product was obtained with cesium carbonate and toluene as the base and solvent respectively. Substitution of the base for potassium fluoride and solvent for dimethoxyethane, however, afforded the desired product in poor yield (16.4\% yield). 


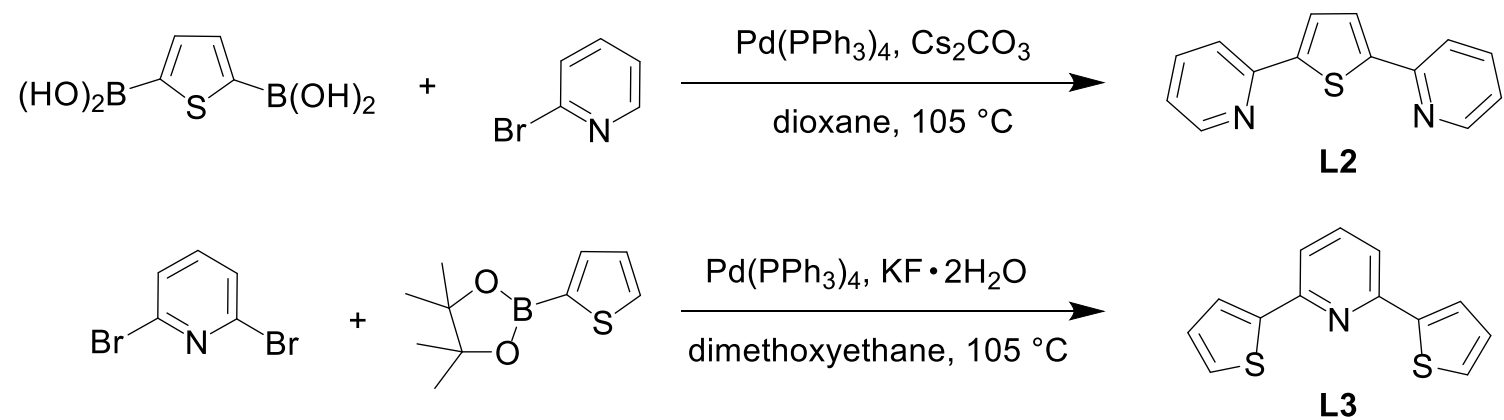

Scheme 4: Synthetic scheme for the synthesis of $\mathbf{L 2}$ and $\mathbf{L 3}$.

For all ligands pyridine coordination to a metal center is thought to facilitate sulfur coordination by holding the metal in close proximity to the sulfur of thiophene. $\mathbf{L} \mathbf{1}$ has one thiophene and one pyridine and is commercially available for purchase. $\mathbf{L 2}$ and $\mathbf{L 3}$ were synthesized using modified literature procedures and designed to have several coordination sites in a larger $\pi$-conjugated system to increase metal ion affinity and redshift the absorption and emission properties. ${ }^{84,85}$ Previous attempts to coordinate $\mathbf{L 3}$ to chromium(II), cobalt(II), nickel(II), copper(II), silver(I), and zinc(II) were unsuccessful. ${ }^{84}$ To the best of our knowledge these ligands have not been shown to coordinate to mercury(II). The structures of all three ligands were verified by ${ }^{1} \mathrm{H},{ }^{13} \mathrm{C}$ NMR and mass spectrometry (MS). Density Functional Theory calculations were performed on each of the ligands (Figure 8). Optimized structures of the ligands show that they are planar compounds. The dihedral angles measured from the optimized structures of the ligands

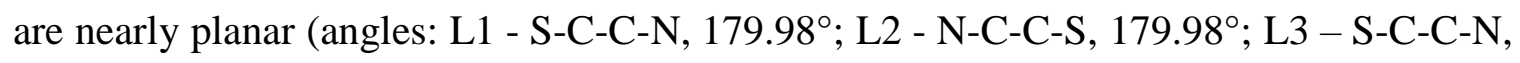
$\left.179.99^{\circ}\right)$. The heteroatoms in each of the rings rotate as shown to prevent ortho-hydrogen interactions. 

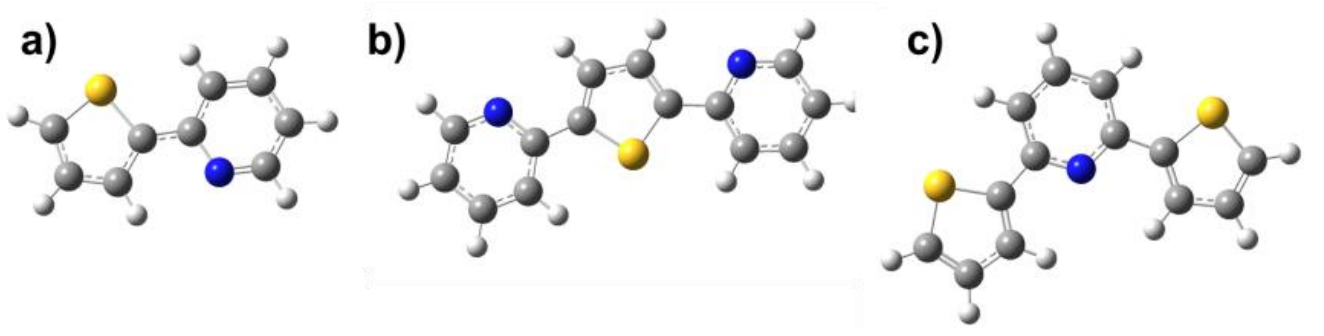

Figure 8: Optimized structures of (a) L1 (b) L2 and (c) L3 (B3LYP /6-311 + G(d)).

\subsubsection{Photophysical Properties of Ligands}

Absorption and emission spectra of $\mathbf{L 1}-\mathbf{L 3}$ were obtained from $1 \times 10^{-5} \mathrm{M}$ solutions in $\mathrm{CH}_{3} \mathrm{CN}$ (Figure 9). Absorption spectra of both $\mathbf{L 2}$ and $\mathbf{L 3}$ are red-shifted relative to $\mathbf{L} \mathbf{1}$ due to extended conjugation from the additional ring. The lowest energy absorption maximum of $\mathbf{L 1}$ is at $298 \mathrm{~nm} ; \mathbf{L} \mathbf{2}$ and $\mathbf{L} \mathbf{3}$ have peaks at 342 and $335 \mathrm{~nm}$, respectively (Table 2). Likewise the emission of both $\mathbf{L} 2\left(\lambda_{\max }=394, \phi=0.56\right)$ and $\mathbf{L} \mathbf{3}$ $\left(\lambda_{\max }=376, \phi=0.34\right)$ are red-shifted compared to that of $\mathbf{L} \mathbf{1}\left(\lambda_{\max }=356 \mathrm{~nm}, \phi=0.43\right)$. Extending the conjugation to three rings pushes the emission into the visible region, providing easy detection of metal binding through visual inspection. 

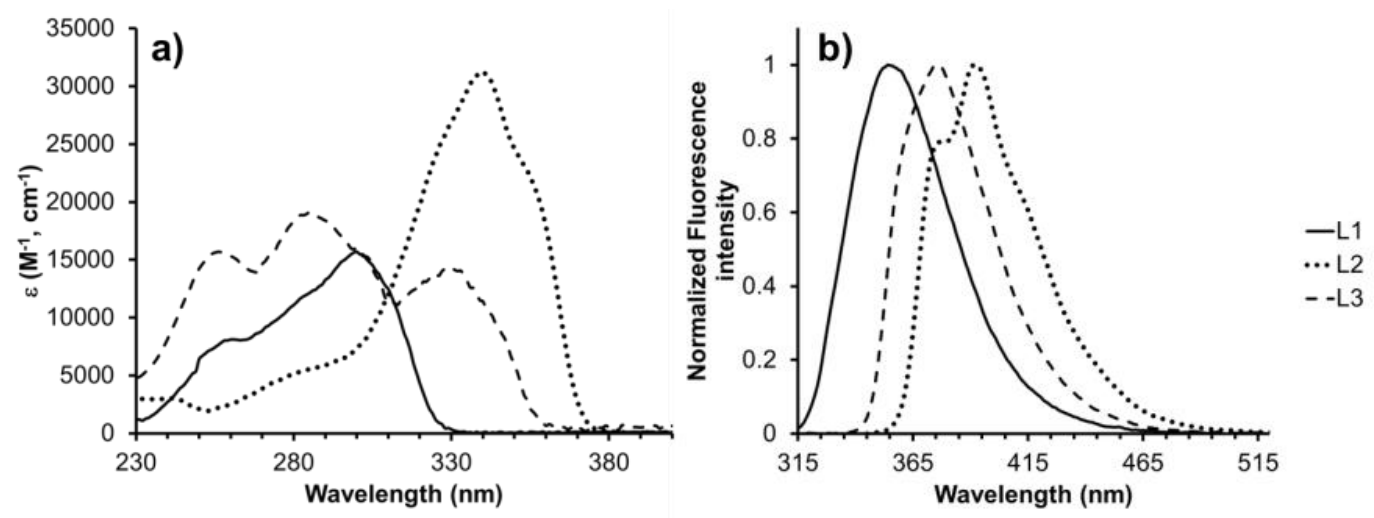

Figure 9: (a) Absorption spectra of L1 (solid), L2 (dots), and L3 (dashes) (b) Emission spectra of $\mathbf{L 1}\left(\lambda_{\mathrm{ex}}=297 \mathrm{~nm}\right.$, solid $), \mathbf{L} \mathbf{2}\left(\lambda_{\mathrm{ex}}=340 \mathrm{~nm}\right.$, dots $)$ and $\mathbf{L 3}\left(\lambda_{\mathrm{ex}}=297 \mathrm{~nm}\right.$, dashes).

Measured absorbance spectra are consistent with calculated excitation energies determined by TD-DFT. The calculated lowest energy absorption for $\mathbf{L} \mathbf{1}$ is at $338 \mathrm{~nm}$ and the calculated lowest energy absorption for $\mathbf{L} \mathbf{2}$ is red shifted to $355 \mathrm{~nm}$. For both $\mathbf{L} \mathbf{1}$ and L2 the lowest energy excitation is $\pi$ to $\pi^{*}$ in nature. The calculated lowest energy peak in calculated absorption spectra of $\mathbf{L 3}$ comprises three transitions at 332, 314, and $290 \mathrm{~nm}$. All three excitations are combinations of $\pi$ to $\pi^{*}$ and $n$ to $\pi^{*}$ transitions. Likewise, three excitations are seen in the measured absorption spectra of $\mathbf{L 3}$. 
Table 2: Photophysical properties of L1-L3 and the complexes formed in solution with $\mathrm{Hg}\left(\mathrm{ClO}_{4}\right)_{2}$.

\begin{tabular}{|c|c|c|c|c|c|}
\hline & $\lambda_{\text {abs }}(\mathrm{nm})$ & $\varepsilon \times 10^{4}\left(\mathrm{M}^{-1} \cdot \mathrm{cm}^{-1}\right)$ & $\lambda_{\text {ex }}(\mathbf{n m})$ & $\lambda_{\text {em }}(\mathbf{n m})$ & $\phi$ \\
\hline L1 & 298 & 1.50 & 304 & 356 & 42.8 \\
\hline $\operatorname{Hg}(\mathbf{L} 1)$ & 335 & 1.71 & 344 & 410 & 79.1 \\
\hline $\mathbf{L} 2$ & 342 & 2.90 & 343 & 394 & 56.1 \\
\hline $\operatorname{Hg}(\mathbf{L} 2)$ & 357 & 3.15 & 360 & 417 & 22.0 \\
\hline $\mathrm{Hg}(\mathrm{L} 2)_{2}$ & 369 & 4.55 & 374 & 435 & 54.7 \\
\hline L3 & 284 & 2.00 & 291 & 376 & 34.4 \\
\hline $\operatorname{Hg}(\mathbf{L 3})$ & 380 & 1.78 & 377 & 457 & 48.2 \\
\hline
\end{tabular}

\subsubsection{Photophysical Response to Mercury}

A summary of the change in photophysical characteristics of each ligand in response to mercury(II) is reported in Table 2. In the presence of mercury(II) ions $\left(\mathrm{Hg}\left(\mathrm{ClO}_{4}\right)_{2}\right)$, the absorption and emission spectra of all three ligands is red-shifted. With increasing equivalents of mercury(II), the ligand-based absorption decreases and a new, red-shifted absorption grows in allowing for ratiometric quantification of the mercury(II) ion. For L1, the absorbance at $298 \mathrm{~nm}$ decreases while a peak at $322 \mathrm{~nm}$ grows until 50 
equivalents of mercury(II) have been added (Figure 10a). An isosbestic point is observed at $314 \mathrm{~nm}$. Likewise, the emission of $\mathbf{L 1}$ at $356 \mathrm{~nm}$ is quenched with addition of mercury(II) and a new peak at $411 \mathrm{~nm}(\phi=0.79)$ appears (Figure 10b).

Although L2 displays a similar red-shift in both absorption and emission, several distinct features are noted. With increasing amounts of mercury(II), $\lambda_{\max }$ of the absorption at $342 \mathrm{~nm}$ decreases as a new peak at $368 \mathrm{~nm}$ increases until 20.0 eq of mercury(II) have been added (Figure 10c). Similarly, a new emission at $446 \mathrm{~nm}(\phi=0.55)$ is observed (Figure 10d). Further additions of mercury(II) past 20.0 eq cause a blue shift in absorption to $360 \mathrm{~nm}$ (Figure 10e) and in emission to $421 \mathrm{~nm}(\phi=0.22)$ (Figure 10f). A clear isosbestic point is not observed for $\mathbf{L} \mathbf{2}$ suggesting the formation of more than one new species in solution.

Addition of mercury(II) to $\mathbf{L} \mathbf{3}$ also causes a red-shift in both the absorption and emission, however 100 eq of metal are required to reach saturation (Figure 10g-h). An isosbestic point at $344 \mathrm{~nm}$ indicates that no intermediate forms during the reaction (Figure 10g). For all three compounds, addition of excess mercury(II) is required to completely quench ligand emission, suggesting that L1-L3 have reasonably low binding affinities for mercury(II). The emission of the complexes also have higher quantum yield than the free ligand. Likely, this results from suppression of nonradiative pathways caused by decreased rotation around the single bond connecting the rings due to metal chelation. 
Binding constants $\left(K_{\mathrm{a}}\right)$ for $\mathbf{L 1}$-L3 were determined using fitting software designed by Dr. Thordarson. ${ }^{79}$ In order to determine the association constants absorption titration data was fit to different models of ligand:mercury ratios (1:1,2:1, and 1:2). In the case of L2 the fit was only performed at low concentrations of mercury(II). Error analysis of the residuals plots was used to eliminate models and determine the most likely stoichiometries of each complex. According to the fitting analysis $\mathbf{L 1}$ and $\mathbf{L 3}$ each bind in a $1: 1$ stoichiometry with a $K_{\mathrm{a}}$ of $7.17 \times 10^{3} \mathrm{M}^{-1}( \pm 6.5 \%)$ and $3.80 \times 10^{3} \mathrm{M}^{-1}$ $( \pm 10.7 \%)$ respectively. $\mathbf{L} 2$ appears to bind in a 2:1 stoichiometry and has an overall $K_{\mathrm{a}}$ of $5.37 \times 10^{9} \mathrm{M}^{-2}( \pm 9.8 \%)$. Likely, the first complex formed during mercury(II) titrations with $\mathbf{L} \mathbf{2}$ is the $2: 1$ product $\left(\operatorname{Hg}(\mathbf{L} 2)_{2}\right)$ due to the high concentration of $\mathbf{L} \mathbf{2}$ relative to mercury(II). This is consistent with the observed stepwise binding constants for the 1:1 $\left(K_{11}=6.93 \times 10^{1} \pm 4 \%\right)$ and $2: 1\left(K_{21}=7.70 \times 10^{7} \pm 9 \%\right)$ complex. As expected $\mathbf{L} \mathbf{2}$ has a relatively higher binding constant relative to $\mathbf{L} \mathbf{1}$ due to the additional chelating group. Surprisingly though $\mathbf{L} \mathbf{3}$ is a tridentate ligand, this sensor has a lower $K_{\mathrm{a}}$ than that of L1. As discussed in Section 3.2.4 of this Chapter, likely the low $K_{\mathrm{a}}$ is due to abstraction of a single thienyl hydrogen as observed via ${ }^{1} \mathrm{H}$ NMR spectroscopy. 

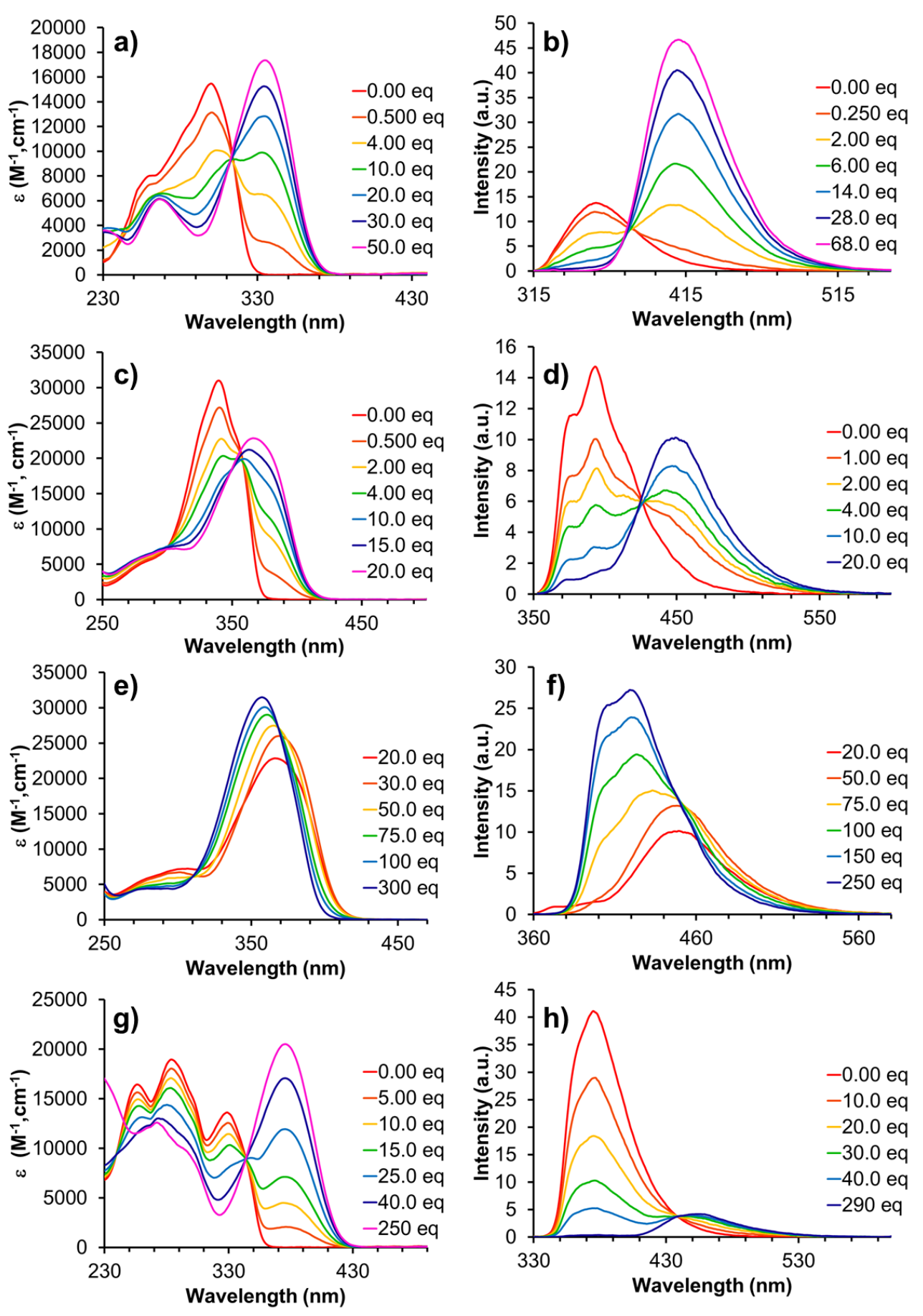

Figure 10: Change in (a) absorption of $\mathbf{L 1}$ (isobestic point $=314 \mathrm{~nm}$ ), (c) absorption of $\mathbf{L} \mathbf{2}$ to 20.0 eq, (e) absorption of $\mathbf{L} \mathbf{2}$ to 300 eq, (g) absorption of $\mathbf{L 3}$ (isobestic point $=344$ $\mathrm{nm})$, (b) emission of $\mathbf{L} \mathbf{1}\left(\lambda_{\mathrm{ex}}=297 \mathrm{~nm}\right)$, (d) emission of $\mathbf{L} \mathbf{2}\left(\lambda_{\mathrm{ex}}=340 \mathrm{~nm}\right)$ to $20.0 \mathrm{eq}$, (f) 
emission of $\mathbf{L} \mathbf{2}\left(\lambda_{\mathrm{ex}}=340 \mathrm{~nm}\right)$ to $250 \mathrm{eq}$, (h) emission of $\mathbf{L 3}\left(\lambda_{\mathrm{ex}}=297 \mathrm{~nm}\right)$ with increasing amounts of $\mathrm{Hg}\left(\mathrm{ClO}_{4}\right)_{2}$ in $\mathrm{CH}_{3} \mathrm{CN}$.

\subsubsection{NMR Titrations for Structure Determination}

To elucidate the structure of the L1-L3/mercury(II) complexes, formation of the complexes were monitored using ${ }^{1} \mathrm{H}$ NMR during the metal titrations into solutions of each ligand. Downfield shifts for ligand peaks were attributed to deshielding of the protons due to metal coordination. Upon coordination to the mercury(II) ion a partial positive charge is delocalized on the rings such that the protons para to the coordination site are the most deshielded. ${ }^{86}$

In the simplest case, with increasing amounts of mercury(II), we would expect there to be a decrease in the peak intensity of our compounds and a simultaneous increase in intensity of a new downfield shifted peak. This would suggest direct conversion of the free ligand to a mercury(II)-complex. However, increasing mercury(II) concentration causes the peaks associated with L1-L3 to gradually shift downfield with no change in the intensity or appearance of any new peaks. This gradual change in the chemical shift suggests rapid ligand exchange reactions are occurring.

In the case of $\mathbf{L} \mathbf{1}$ the proton para to the pyridyl nitrogen $\left(\mathrm{H}_{\mathrm{E}}\right)$ (Figure 11a) shifts 0.717 ppm with addition of 2 eq of mercury(II), suggesting nitrogen coordination. $\mathrm{H}_{\mathrm{C}}$ in the 3-position of thiophene was shifted $0.457 \mathrm{ppm}$ relative to the free ligand suggesting possible sulfur-coordination to mercury(II). These results are consistent with S,N-chelation of $\mathbf{L 1}$ to mercury(II), at a 1:1 stoichiometry. 


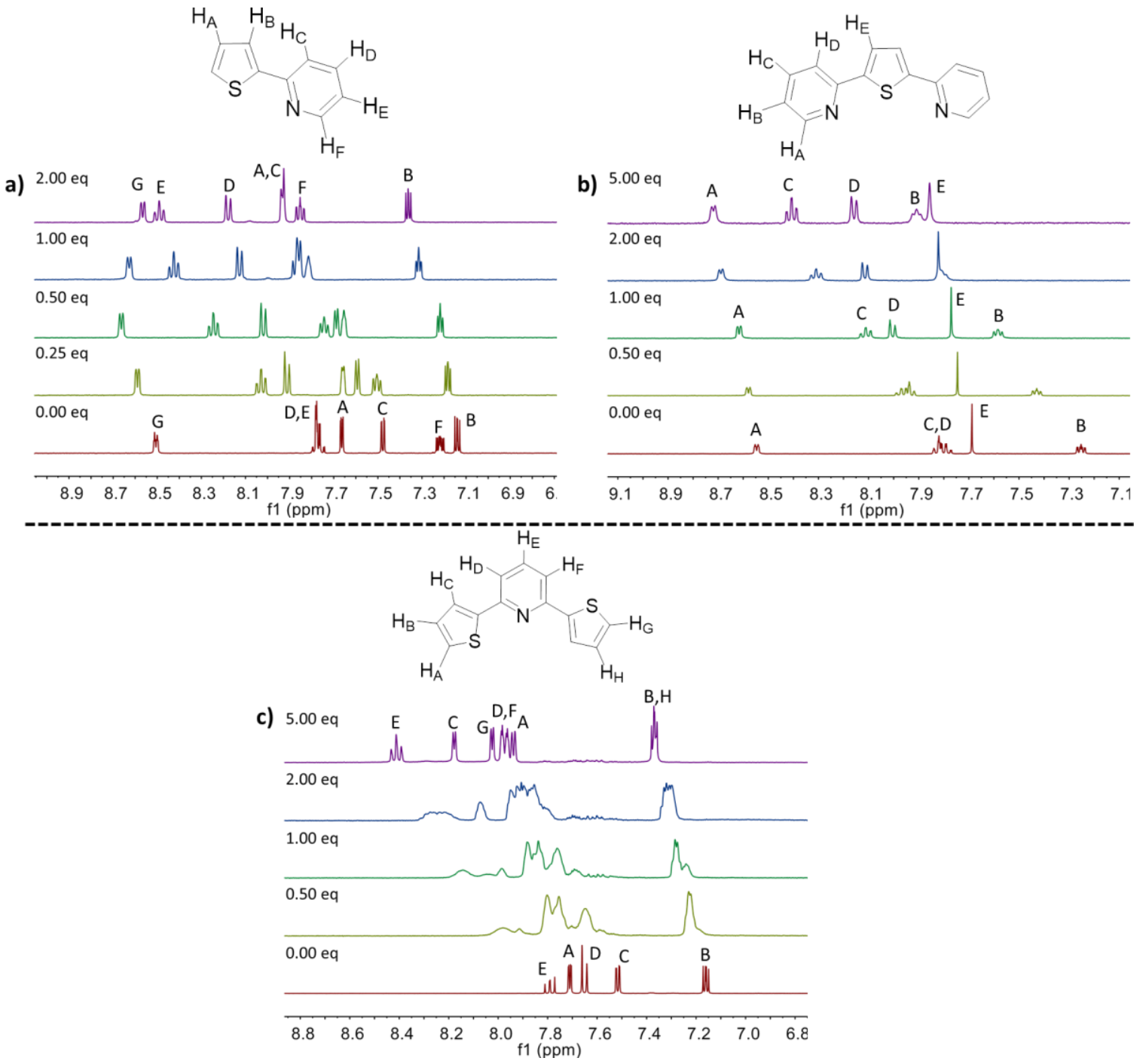

Figure 11: Change in ${ }^{1} \mathrm{H}$ NMR spectrum with increasing amounts of $\mathrm{Hg}\left(\mathrm{ClO}_{4}\right)_{2}$ for (a) $\mathbf{L 1}$, (b) L2, and (c) $\mathbf{L 3}$ in $\mathrm{CD}_{3} \mathrm{CN}$. Proposed structures with labeled protons are overlaid for each spectrum.

Since two new products are formed with addition of mercury(II) to $\mathbf{L} \mathbf{2}$, emission spectroscopy was used in conjunction with ${ }^{1} \mathrm{H}$ NMR to differentiate the two products. The symmetry of the molecule was retained during the titration, indicated by retention of 
the singlet peak from $\mathrm{H}_{\mathrm{E}}$ on the thiophene. After 1.00 eq of mercury(II) was added, emission spectroscopy confirmed the identity of the first product $\left(\lambda_{\max }=435 \mathrm{~nm}\right)$. A low downfield shift $(\Delta \mathrm{ppm}=0.083 \mathrm{ppm})$ is observed for the protons at the 3,4-positions of thiophene $\left(\mathrm{H}_{\mathrm{E}}\right)$, while a $0.471 \mathrm{ppm}$ shift was observed for the proton para to the pyridyl nitrogen $\left(\mathrm{H}_{\mathrm{C}}\right)$ (Figure $\left.11 \mathrm{~b}\right)$. After $5 \mathrm{eq}$ an emission at $420 \mathrm{~nm}$ indicated the second product had formed in solution. A greater downfield shift for all peaks was observed. The symmetry of the molecule must be retained throughout the titration, as the number of peaks is consistent throughout. This suggests the identity of the complexes formed in solution are the result of $\mathrm{N}, \mathrm{S}, \mathrm{N}$ chelation the $1: 2$ complex $\left(\operatorname{Hg}(\mathbf{L} 2)_{2}\right)$ followed by subsequent formation of the 1:1 complex $(\operatorname{Hg}(\mathbf{L 2}))$ via chelation of the same heteroatoms.

During the titration of $\mathbf{L} \mathbf{3}$ with mercury(II), a broadening of all peaks was observed until 5.00 eq of mercury(II) were added and the product was formed. Unlike titrations with $\mathbf{L} \mathbf{1}$ and $\mathbf{L 2}$, the total number of aromatic protons decreased by one after the addition of mercury(II). Another distinct feature is an apparent break in symmetry observed by an increase in the number of proton environments (Figure 11c). Following addition of 5 eq of mercury(II), the ${ }^{1} \mathrm{H}$ NMR spectrum suggests a proton at the 3-position of one of the thiophenes had been removed from the ligand. Removal of this proton indicates S,N,Cchelation by $\mathbf{L 3}$ to mercury(II) at a 1:1 stoichiometry. This is the only ligand that appears to coordinate through a carbon rather than sulfur. 


\subsubsection{Selectivity towards Mercury(II)}

The selectivity of the fluorescent response was tested against other metal perchlorate salts: mercury(II), silver(I), cadmium(II), cobalt(II), copper(II), iron(II), iron(III), magnesium(II), manganese(II), sodium(I), nickel(II), lead(II), and zinc(II). The emissions of solutions with 5 eq of each metal salt were measured. For L1, only addition of mercury(II), copper(II), and iron(III) resulted in an emission response. The presence of other metal ions in solution did not affect the emission response to mercury(II), with the exception of iron(III) which showed an enhanced emission (Figure 12a). Similar selectivity was observed for L2. There is a bathochromic shift for 5 eq of iron(III) $\left(\lambda_{\max }=420 \mathrm{~nm}\right)$ relative to the free $\mathbf{L} \mathbf{2}$. However, addition of mercury(II) alone results in a different emission $\left(\lambda_{\max }=446 \mathrm{~nm}\right)$ (Figure 12b). Notably, L3 emission was only slightly quenched in response to 5 eq of all metal salts, including mercury(II). iron(III) addition caused a new emission at $457 \mathrm{~nm}$ (Figure 12c). These results indicate that thiophene containing fluorescent sensors can display selectivity for mercury(II). 

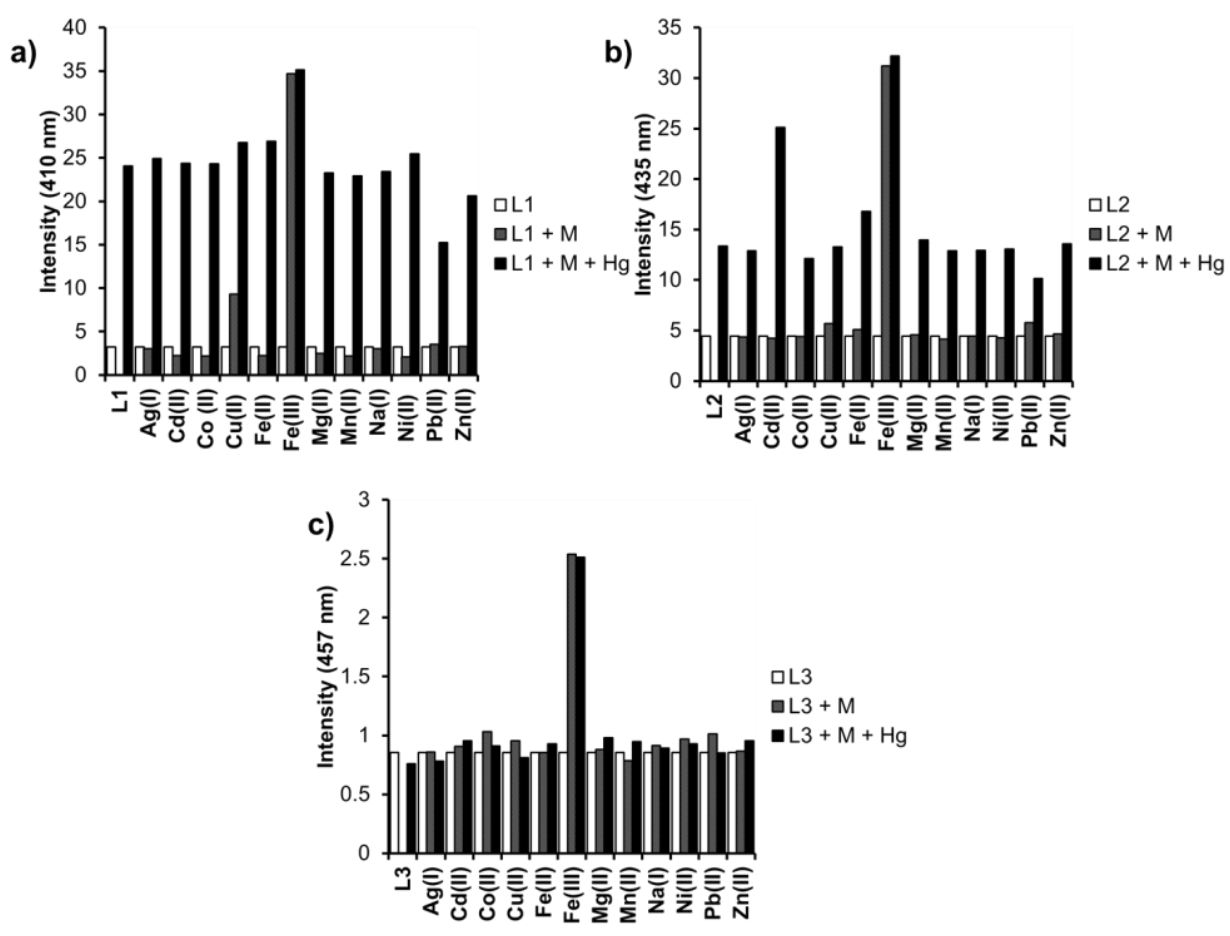

Figure 12: Emission of (a) L1, (b) L2, and (c) $\mathbf{L 3}$ at 410, 435, and $457 \mathrm{~nm}$, respectively, before addition of metal (white bars), after the addition of 5 eq. of metal (grey bars), and after addition of 5 eq. of $\mathrm{Hg}\left(\mathrm{ClO}_{4}\right)_{2}$ in the presence of 5 eq. of metal salt (black bars).

\subsection{Conclusion}

We have developed three new thiophene-based ligands with a fluorescence response to mercury(II) via chelation to the metal center. The addition of pyridyl groups appears to enhance coordination of the thienyl sulfur via chelation, as is evident through the greater association constant observed for $\mathbf{L} \mathbf{2}$, which contains two pyridines, relative to the association constants of $\mathbf{L 1}$ and $\mathbf{L 3}$, both containing only one pyridine. This suggests that the sulfur of thiophene is not a strong coordinating group. A unique coordination mode is observed via ${ }^{1} \mathrm{H}$ NMR for $\mathbf{L 3}$ which suggests a S,N,C-chelation to mercury(II). Our results indicate that thiophene ligands offers selectivity for mercury(II). A unique 
coordination chemistry is observed for $\mathbf{L} \mathbf{2}$ and mercury(II), forming two distinct products dependent on the amount of mercury(II) in solution. Based on association constant the two products formed appear to be have stoichiometries of 1:2 and 1:1 (mercury:L2) which chelate via the pyridyl nitrogen and thienyl sulfur atoms. In conclusion, we have shown that thiophene containing ligands display selectivity for mercury(II) giving a visible, ratiometric fluorescence response. 


\section{Chapter 4. Dibenzothiophene-Based Ligands as Chemical Sensors for Mercury(II)}

\subsection{Introduction}

In the previous chapter a set of sensors that incorporated both thiophene and pyridine rings was reviewed. These sensors were shown to have a ratiometric photophysical response and reasonable selectivity for mercury(II). Unfortunately the $K_{\mathrm{a}}$ values were less than ideal to be applicable as mercury(II) sensors. In an effort to improve the affinity for the mercury(II) atom the thiophene ring was substituted for dibenzothiophene, a ligand that has been previously shown to have a greater association constant for transition metals relative to thiophene. In addition to modification of the thiophene ring we chose to vary the chelating group as well, to determine an ideal chelating group for the mercury(II) ion. Beyond the modifications mentioned the chelate ring size has also increased with the sensors of this study which could lower the affinity for the large mercury(II) ion. In this study four ligands were developed including; 2(dibenzo[b,d]thiophen-4-yl)pyridine (BT1), 2-(dibenzo[b,d]thiophen-4-yl)-1H-imidazole (BT2), and 2-(dibenzo[b,d]thiophen-4-yl)thiazole (BT3) that contain pyridine, imidazole, and thiazole chelating groups respectively. The focus of this work is to both determine whether dibenzothiophene provides an improvement in $K_{\mathrm{a}}$ from thiophene and evaluate the changes in both selectivity and $K_{\mathrm{a}}$ upon substitution of the pyridyl chelating group for other nitrogen and sulfur donors. 


\subsection{Results}

\subsubsection{Synthesis and Structure of BT1-BT3}

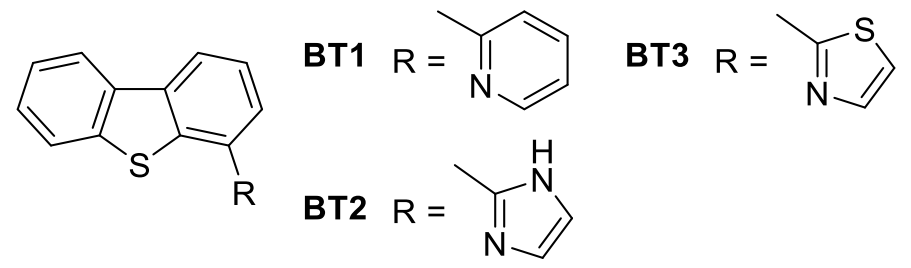

Figure 13: Structural depiction of BT1-BT3.

The sensors for this project are designed as bidentate ligands containing a dibenzothiophene moiety coupled at the 4-position to a pyridine (BT1), imidazole (BT2), or thiazole (BT3) (Figure 13). These ligands were designed based on L1, the only bidentate ligand described in the previous chapter. BT1 contains a single pyridyl chelating group, similar to $\mathbf{L} \mathbf{1}$, to evaluate the effects of substituting the thiophene ring for dibenzothiophene. A mix of nitrogen and sulfur donors have also been incorporated to assess different chelating groups selectivity and affinity for the mercury(II) ion, in comparison to pyridine. In doing so I hope to determine other potentially useful chelating groups towards the development of a selective mercury(II) sensor. 

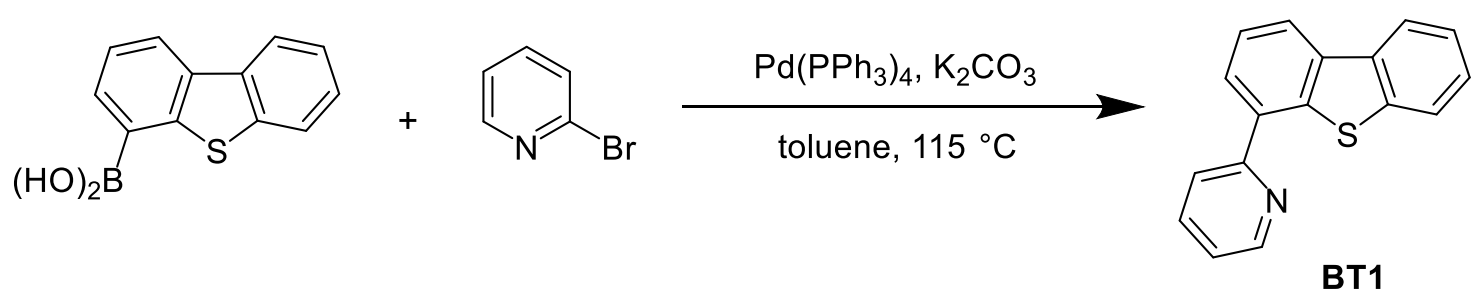

BT1

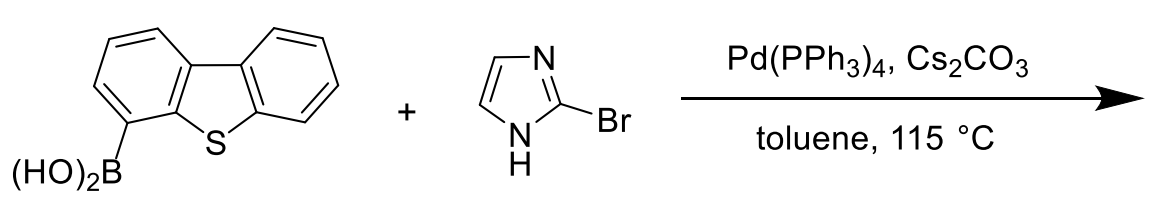<smiles>Brc1cnc(-c2cccc3c2sc2ccccc23)[nH]1</smiles><smiles>O=C(O)c1cccc2c1sc1ccccc12</smiles><smiles>c1ccc2c(c1)sc1c(-c3nccs3)cccc12</smiles>

Scheme 5: Synthetic scheme for the synthesis of BT1-BT3.

All sensors for this project were synthesized in single-step Suzuki coupling reactions joining the dibenzothiophene moiety to the corresponding heterocycle (Scheme 5). Previously reported procedures ${ }^{87}$ were used to synthesize BT1 in $82.3 \%$ yield. Compounds BT2 and BT3 were obtained following custom Suzuki coupling procedures described in Section 2.2.4-2.2.5 of Chapter 2 in $88.6 \%$ and $54.6 \%$ yield respectively. BT1 is the only compound that has previously been incorporated as a ligand to a ruthenium(II) metal center. However this was primarily for the purpose of developing a thiolate pincer ligand, rather than sensing applications. ${ }^{87}$ To the best of my knowledge none of these ligands have been previously tested as chemical sensors. 


\subsubsection{Photophysics of Dibenzothiophene ligands}
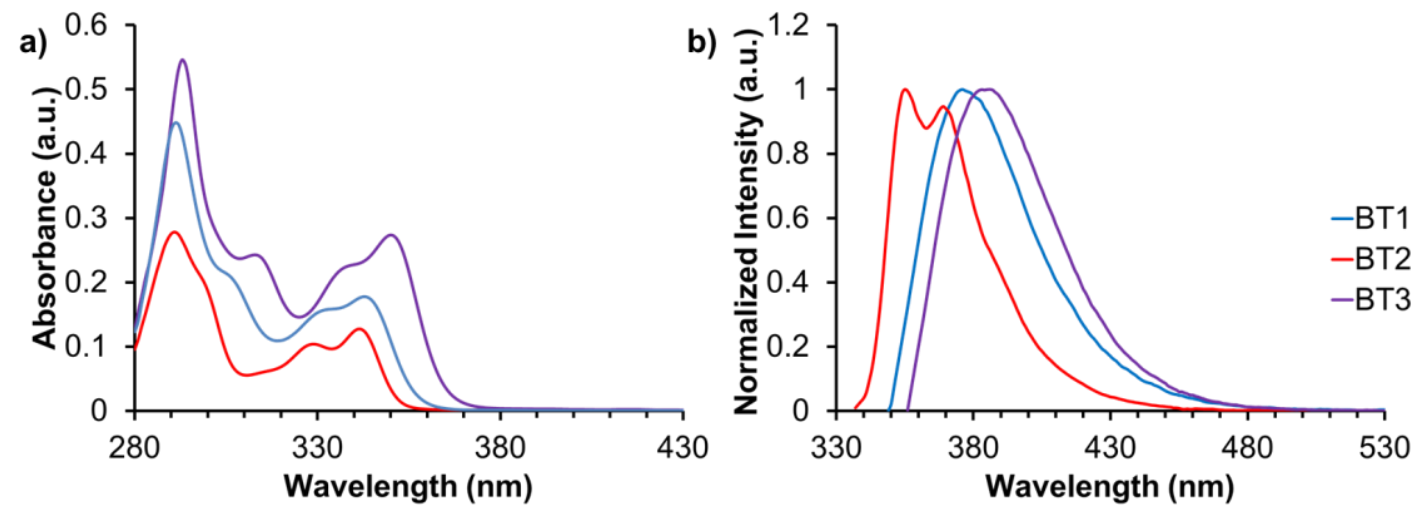

Figure 14: Absorption (a) and emission (b) spectrum of BT1-BT3 in $\mathrm{CH}_{3} \mathrm{CN}$ at $3 \times 10^{-5} \mathrm{M}$.

The photophysics of BT1-BT3 were obtained at $3 \times 10^{-5} \mathrm{M}$ in $\mathrm{CH}_{3} \mathrm{CN}$. To ensure proper resolution was obtained the concentration of all ligands was increased relative to sensors described in other chapters of this document. A comparison of L1 $\left(\varepsilon=1.50 \times 10^{4} \mathrm{M}^{-1} \cdot \mathrm{cm}^{-1}\right)$ and BT1 $\left(\varepsilon=5.92 \times 10^{3} \mathrm{M}^{-1} \cdot \mathrm{cm}^{-1}\right)$ reveals a dramatic decrease in absorptivity in the visible region upon substitution of the thiophene ring for dibenzothiophene. This is evident for all ligands as the absorption intensity for BT1-BT3 is lower than all sensors described in other chapters. The highest intensity absorption peaks are observed in the UV-region for all sensors (BT1: $291 \mathrm{~nm}$; BT2: $291 \mathrm{~nm}$; BT3: $293 \mathrm{~nm}$ ). This was not expected based on the extend $\pi$-system of these sensors, however the low-energy absorptions summarized in Table 3 are all red-shifted relative to $\mathbf{L 1}\left(\lambda_{\max }=298 \mathrm{~nm}\right)$. In the case of BT2 the observed emission wavelength is close to that of L1 $\left(\lambda_{\max }=356 \mathrm{~nm}\right)$, while the emission of BT1 and BT3 are red-shifted in comparison (Table 3). Given all dibenzothiophene ligands have quantum yields close to $1 \%$, 
substitution of the thiophene ring for dibenzothiophene results in a dramatic decrease in quantum yield.

Table 3: Summary of photophysical data for BT1-BT3 obtained in $\mathrm{CH}_{3} \mathrm{CN}$ at $3 \times 10^{-5} \mathrm{M}$ concentration. Reported absorption wavelengths and molar absorptivities are those in the visible region.

$$
\lambda_{\text {abs }}(\mathrm{nm}) \quad \varepsilon \times 10^{3}\left(\mathrm{M}^{-1} \cdot \mathrm{cm}^{-1}\right) \quad \lambda_{\mathrm{ex}}(\mathrm{nm}) \quad \lambda_{\mathrm{em}}(\mathrm{nm}) \quad \phi(\%)
$$

\begin{tabular}{llllll}
\hline BT1 & 343 & 5.92 & 345 & 376 & 1.2 \\
BT2 & 341 & 4.24 & 330 & 355 & 1.0 \\
BT3 & 350 & 9.12 & 340 & 386 & 1.9 \\
\hline
\end{tabular}

\subsubsection{Photophysical Response to $\mathrm{Hg}^{2+}$}

The photophysical response of BT1-BT3 to mercury(II) was examined in $\mathrm{CH}_{3} \mathrm{CN}$. Absorption and emission titrations were performed with ligand concentration at $3 \times 10^{-5}$ M. Multiple products were observed for both BT1 and BT2 (Figure 15). Similar changes in absorption and emission spectrum are observed in both cases, however some distinctions are noted. In both cases low concentrations of mercury(II) result in a decrease in absorbance intensity and blue-shift in the absorbance spectrum. In the case of BT1 a red-shifted shoulder also arises allowing for ratiometric detection. Greater concentrations result in a red-shifted peak growing in until saturation at greater than 100 equivalents of mercury(II). 

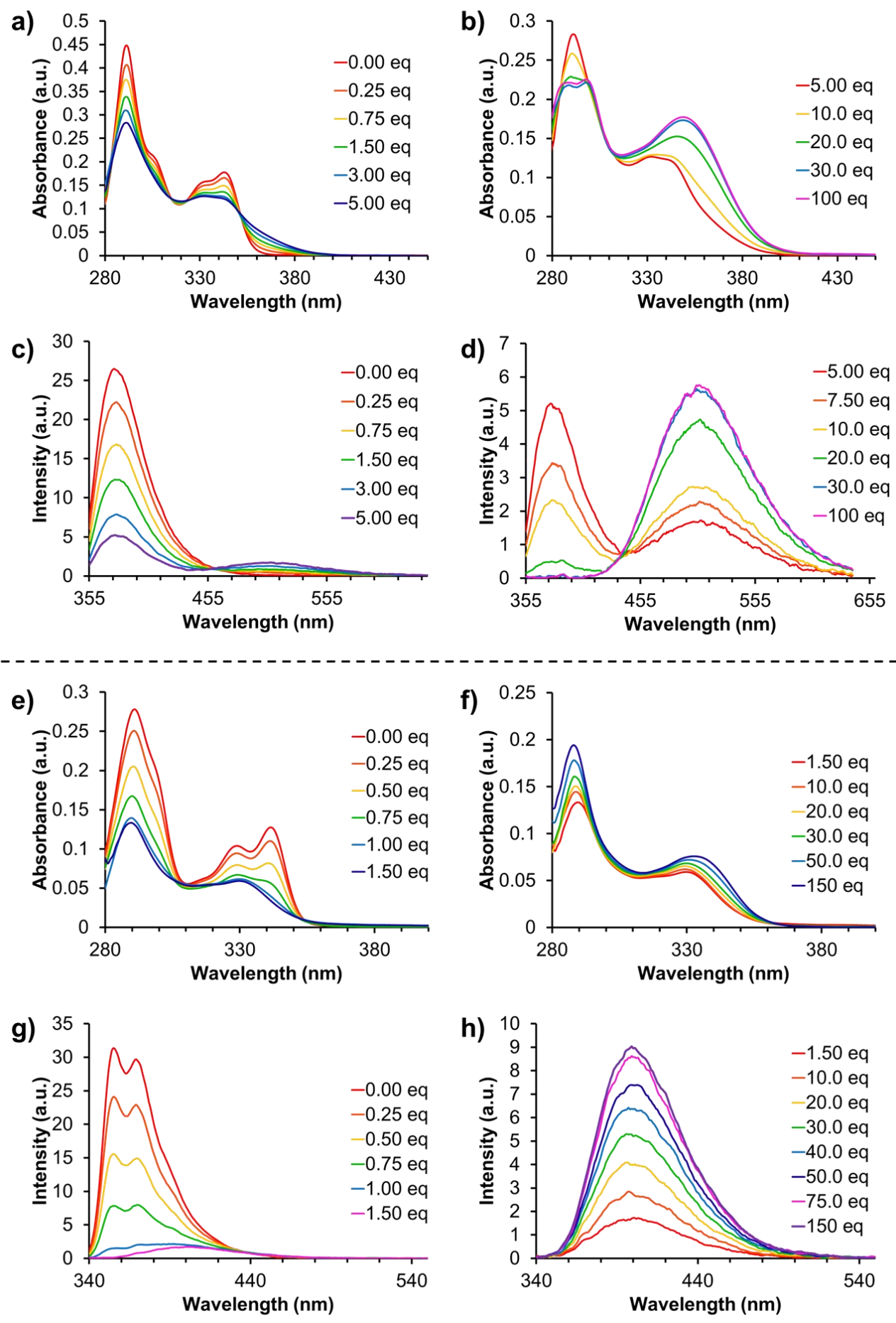

Figure 15: Absorption (a-b \& e-f) and emission (c-d \& g-h) titrations of BT1 and BT2 respectively with increasing concentrations of mercury(II) in $\mathrm{CH}_{3} \mathrm{CN}$ at $3 \times 10^{-5} \mathrm{M}$. Emission spectra were obtained by excitation at $345 \& 330 \mathrm{~nm}$ respectively. 
The initial emission response to mercury(II) for BT1 and BT2 is quenching of the sensor emission. In the case of BT1 a red-shifted shoulder also grows in with greater amounts, while for BT2 a bathochromic shift in $\lambda_{\max }$ is observed. Larger concentrations of mercury result in an increase in intensity of a new red-shifted peak. The lack of an isosbestic point in both cases suggests multiple products are formed throughout the titration. In the case of BT1 this is observed in the emission spectrum at 3.00 equivalents of mercury(II), when a new red-shifted peak begins to grow in. Likely the first product has no emission, and the observed emission is due to the free ligand and the second product being formed resulting in the two peaks observed in the emission spectrum.
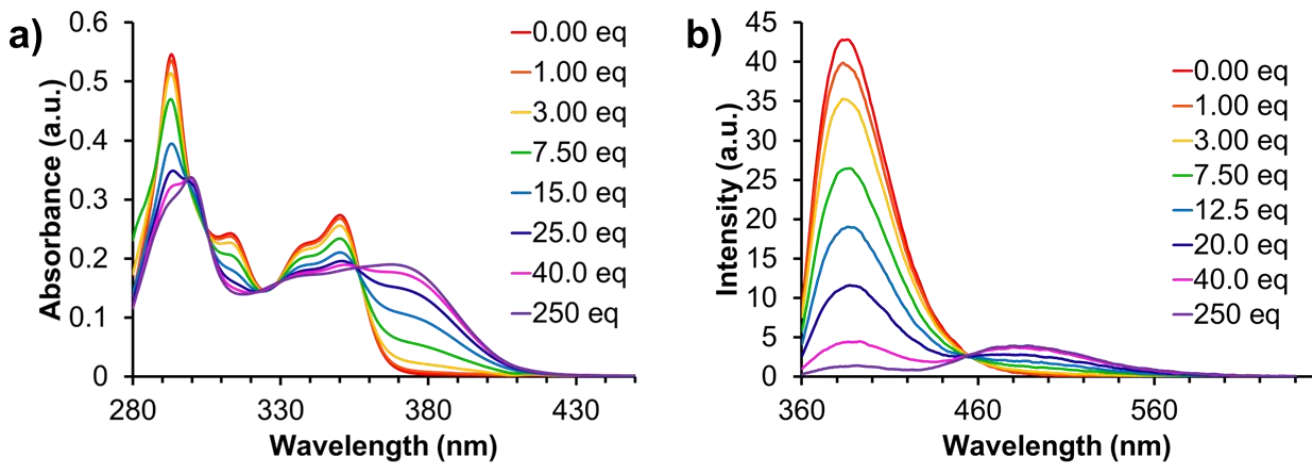

Figure 16: Change in (a) absorption and (b) emission of BT3 in response to increasing amounts of mercury(II) in $\mathrm{CH}_{3} \mathrm{CN}$ at $3 \times 10^{-5} \mathrm{M}$. Emission spectra were obtained by excitation at $350 \mathrm{~nm}$.

For BT3 increasing amounts of mercury(II) result in ratiometric red-shift in both absorption and emission $\lambda_{\max }$ (Figure 16). Two isosbestic points are observed during the absorption titration of BT3 suggesting direct conversion from the starting material to product (Figure 16a). All sensors from this project appear to have relatively low binding affinity for mercury(II), as all of them require at least 100 equivalents to generate the 
final products in solution. BT1-BT3 have useful photophysical changes since addition of mercury(II) eventually results in a red-shift in both the absorption and emission towards the visible region. Unfortunately though a red-shift in the absorption is observed in these cases no color change was observed at the concentrations tested, likely due to low molar absorptivities of the formed complexes.

Table 4: Overall association constants of BT1-BT3 obtained from absorption titration data in $\mathrm{CH}_{3} \mathrm{CN}$.

\begin{tabular}{cc}
\hline Sensor & $\boldsymbol{K}_{\mathrm{a}}$ \\
\hline BT1 & $3.73 \times 10^{8} \mathrm{M}^{-2}( \pm 18.7 \%)$ \\
BT2 & $1.67 \times 10^{8} \mathrm{M}^{-2}( \pm 28.1 \%)$ \\
BT3 & $2.17 \times 10^{6} \mathrm{M}^{-2}( \pm 2.51 \%)$ \\
\hline
\end{tabular}

The goal of this project was to determine whether substitution of the thiophene moiety of $\mathbf{L} \mathbf{1}$ for dibenzothiophene afforded greater sulfur binding affinity. Furthermore the pyridyl chelating group was varied to evaluate whether different chelating groups would afford greater selectivity and $K_{\mathrm{a}}$. As such association constants for BT1-BT3 were determined from absorption titration data and compared (Table 4). A comparison of the $K_{\mathrm{a}}$ values of BT1 and $\mathbf{L 1}\left(K_{\mathrm{a}}=7.17 \times 10^{3} \mathrm{M}^{-1} \pm 6.5 \%\right)$ show a great increase in $K_{\mathrm{a}}$ for mercury(II) upon substituion of thiophene for dibenzothiophene. Of the sensors BT1 also had the highest $K_{\mathrm{a}}$ value offering good evidence that pyridine is the ideal chelating group 
over those tested. Interestingly BT1-BT3 fit to a 2:1 stoichiometry of sensor to metal, distinct from $\mathbf{L} \mathbf{1}$ described in the previous chapter which fit to a 1:1 model. Though the absorption titration data of BT3 fit to a 2:1 model no second product was observed at even high concentrations of mercury(II). Likely this is due to a low $K_{\mathrm{a}}$ value for the corresponding 1:1 product.

\subsection{4 ${ }^{1}$ H NMR Experiments for Structure Determination}

To further investigate the coordination events occurring with these sensors ${ }^{1} \mathrm{H}$ NMR experiments were performed. Addition of mercury(II) metal to solutions of BT1BT3 result in a shift of the protons in the ${ }^{1} \mathrm{H}$ NMR spectrum. In all cases initial addition of mercury(II) result in an upfield shift of several peaks which could be due to $\pi$-backbonding of the mercury(II) metal center. Greater concentrations result in a downfield shift of all peaks. These observed shifts are consistent with bidentate chelation for all sensors suggesting coordination to mercury(II) occurs. Broadening of the peaks occurs with increasing concentrations of metal suggesting exchange between different coordination complexes, consistent with previous experiments discussed in Chapter 3. 


\section{a)}

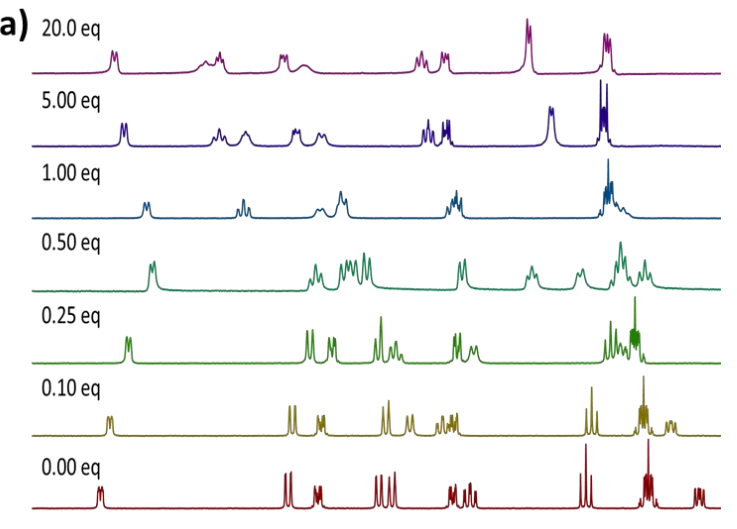

8.98 .88 .78 .68 .58 .48 .38 .28 .18 .07 .97 .87 .77 .67 .57 .4 f1 (ppm)

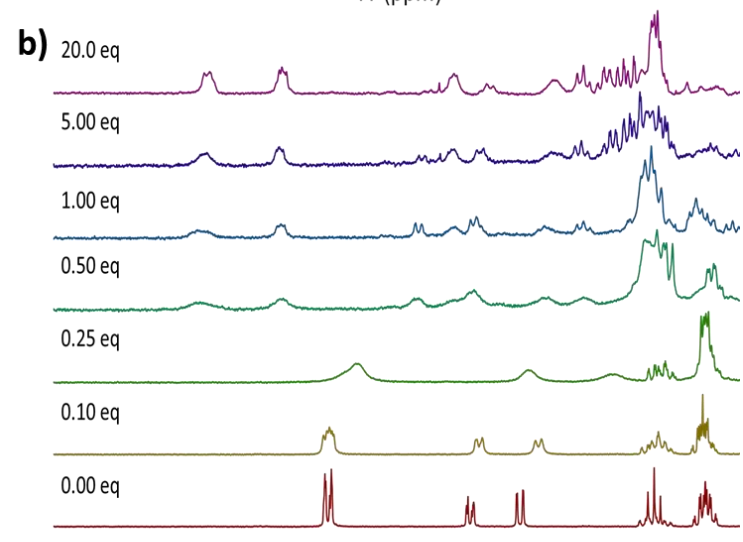

8.808 .708 .608 .508 .408 .308 .208 .108 .007 .907 .807 .707 .607 .50 f1 (ppm)

\section{c)}

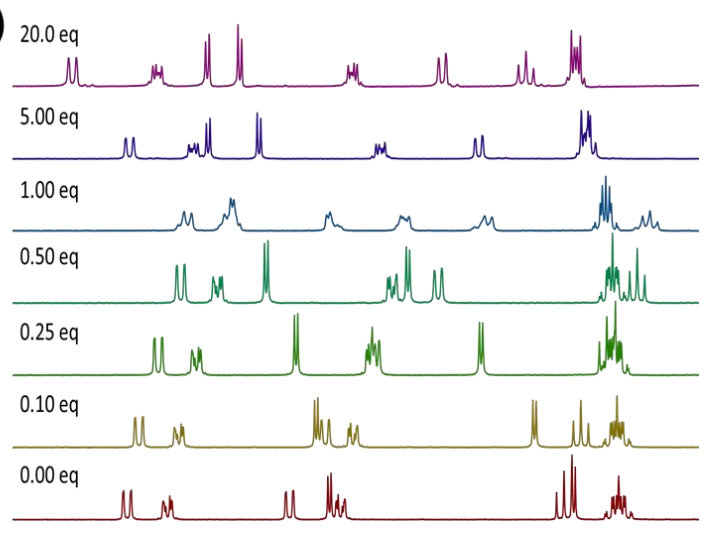

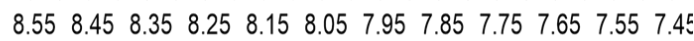
f1 (ppm)

Figure 17: ${ }^{1} \mathrm{H}$ NMR of (a) BT1, (b) BT2, and (c) BT3 with increasing amounts of mercury(II) in $\mathrm{CD}_{3} \mathrm{CN}$ at $4 \times 10^{-3} \mathrm{M}$ of sensor. 


\subsubsection{Selectivity of BT1-BT3 for Mercury(II)}

Competition experiments were performed with BT1-BT3 to determine the selectivity of these sensor for the mercury(II) ion. Of the three sensors BT2 appears to be the least selective for mercury(II) as a response is observed for cadmium(II), copper(II), iron(II), iron(III), lead(II) and zinc(II). Though subsequent addition of mercury(II) results in displacement of several of these metals, competitive binding is still observed with copper(II) and iron(III). In the case of BT1 and BT3 competitive binding appears to occur only with iron(III). However the relatively low $K_{\mathrm{a}}$ of BT3 suggests this sensor would not be applicable as a mercury(II) sensor. In contrast BT1, bearing the pyridyl chelating group affords ideal sensing properties for mercury(II) as it provides the most selective response with greater $K_{\mathrm{a}}$ from this set of ligands. 

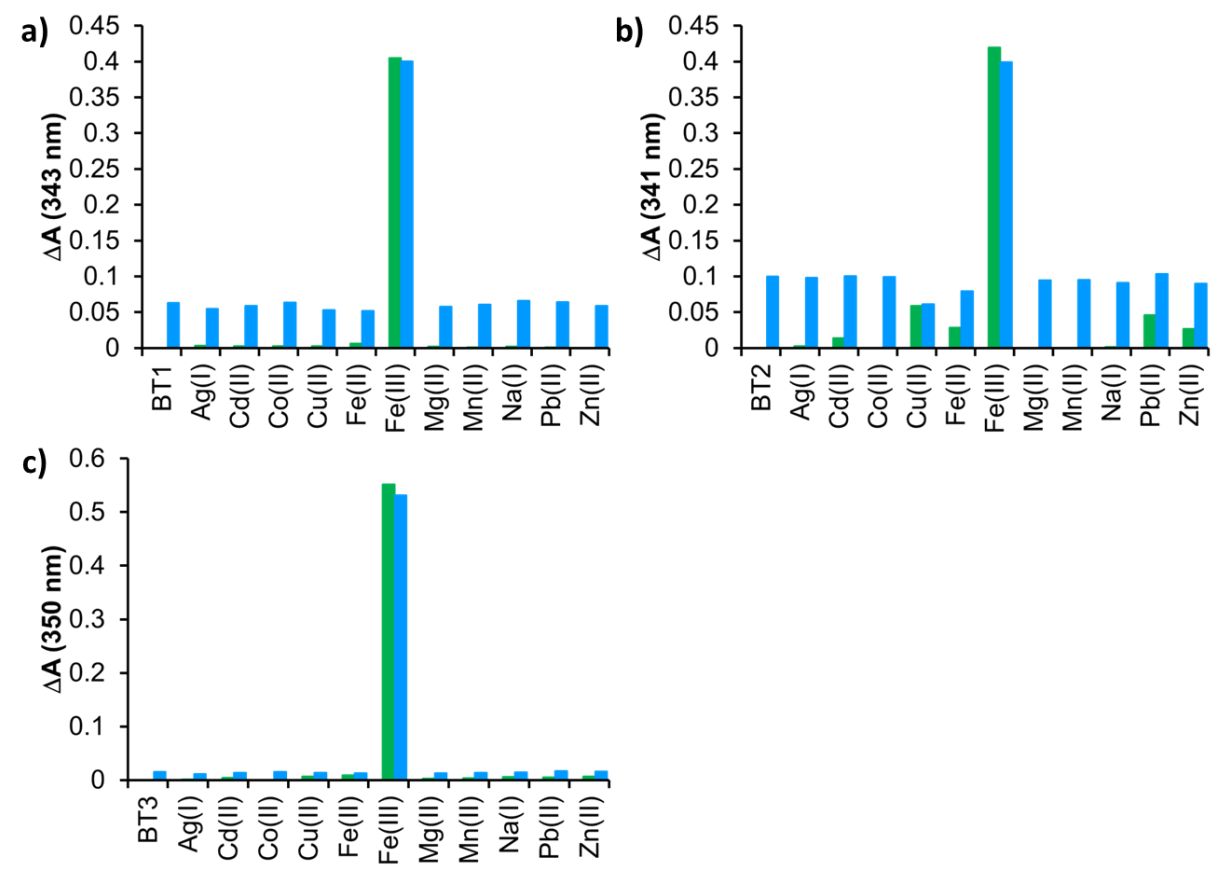

Figure 18: Competition experiments showing the difference in absorptivity for (a) BT1, (b) BT2, and (c) BT3 in the presence of 5 eq of metal salt (green bars) and subsequent addition of 5 eq of mercury(II) (blue bars) in $\mathrm{CH}_{3} \mathrm{CN}$ at $3 \times 10^{-5} \mathrm{M}$ concentration of sensor.

\subsection{Conclusion}

In summary, dibenzothiophene sensors were developed with several different chelating groups. This was done in an effort to increase the $K_{\mathrm{a}}$ of $\mathbf{L 1}$, which contained a thiophene moiety rather than dibenzothiophene, and to evaluate the choice of the pyridyl chelating group. As expected the dibenzothiophene moiety appears to increase the $K_{\mathrm{a}}$ for mercury(II), however less than ideal photophysical characteristics and responses are observed. The thiazole and pyridine groups affords similar selectivity however BT3, bearing the thiazole chelating group, has a relatively lower $K_{\mathrm{a}}$. Therefore the pyridyl chelating group of BT1 appears to be the most ideal of those tested based on the high 
selectivity and $K_{\mathrm{a}}$. BT1 is also one of the two sensors which afforded multiple products with increasing amounts of mercury(II) suggesting it could detect a larger range of concentrations, more ideal for sensing applications. 


\section{Chapter 5. Evaluation of the Effects on Supramolecular Properties by Functionalization of a Thiophene-Based Fluorescent Mercury(II) \\ Sensor}

\subsection{Introduction}

Though the dibenzothiophene ligands were shown to improve the $K_{\mathrm{a}}$, substitution of the thiophene ring afforded less than ideal photophysical characteristics. Furthermore the sensors discussed thus far have only been soluble in organic solvents, limiting their applications in biological systems. In order to both improve the ligand's water solubility and maintain the photophysical characteristics observed from the first generation of sensors we chose to functionalize a previous sensor (L2) with electron-donating groups. L2 was the preferred ligand of choice as it was observed to form two distinct products in solution which would allow for a greater range of concentrations to be detected. Furthermore of the original compounds studied, this was the sensor which was observed to have the highest $K_{\mathrm{a}}$ and was therefore the most ideal choice. For this project the effects of functionalizing the pyridine rings of $\mathbf{L 2}$ were investigated. These sensors have been functionalized at the 3-position of the pyridine rings with alcohol (P1), octaethylene glycol monomethyl ether (P2), or amine (P3) groups to increase water solubility. The photphysical response of these sensors in both organic solvent and mixed aqueous media will be discussed. In addition to this the change in supramolecular properties such as 
coordination number and $K_{\mathrm{a}}$ will be reviewed in comparison to the nonfunctionalized ligand (L2).

\subsection{Results}

\subsubsection{Synthesis and Structure of Pyridine-Functionalized Ligands}

These ligands are based on 2,5-bis(2-pyridyl)thiophene (L2), a fluorescent sensor that was previously discussed in Chapter 3 (Figure 19). ${ }^{83}$ The addition of watersolubilizing functional groups to the 3-position of the pyridyl groups further red-shifts the absorption and emission of the sensors relative to $\mathbf{L 2}$. This results in all sensors from this Chapter to have absorption and emission wavelengths in the visible region, in addition to various other changes in the photophysical properties that will be discussed in the following section.

The compounds have been substituted at the 3-position of both pyridine moieties with water solubilizing functional groups. Compounds $\mathbf{P 1}$ and $\mathbf{P 3}$ are sparingly soluble in pH 7 water (3.46 $\mathrm{mg} \cdot \mathrm{L}^{-1}$ and $2.24 \mathrm{mg} \cdot \mathrm{L}^{-1}$ respectively). The octaethylene glycol monomethyl ether chains of $\mathbf{P 2}$ make the sensor readily soluble in aqueous solution. Water-solubilizing groups were placed in the 3-position to decrease interaction of these groups with the desired coordination site of the thienyl sulfur and pyridyl nitrogen atoms. The water-solubilizing groups are also electron donating in nature thus increasing the electron density of the pyridine ring. 


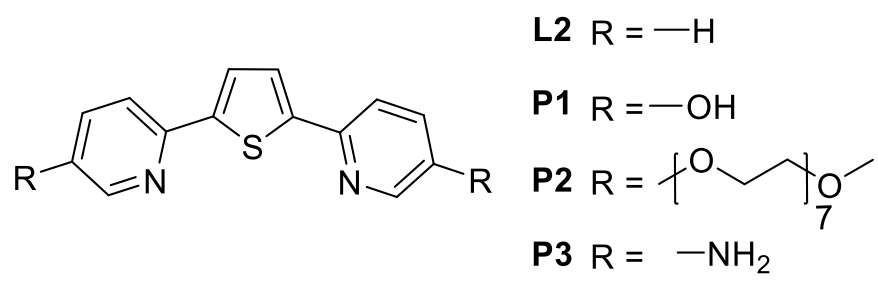

Figure 19: Structural depiction of L2 and P1-P3.

To the best of my knowledge these compounds (P1-P3) have not been synthesized previously. Compounds $\mathbf{P 1}$ and $\mathbf{P 3}$ were synthesized via Suzuki coupling reactions from modified procedures in the literature. ${ }^{85}$ The addition of the water-solubilizing groups had little effect on the isolated yield relative to synthesis of $\mathbf{L} \mathbf{2}$ from $37.1 \%$ to $31.1 \%$ and 38.7\% respectively. Compound P2 was synthesized from P1 following modified literature procedures. ${ }^{88}$ Two separate additions of iodoheptaethyleneglycol monomethyl ether were performed rather than a single addition to ensure full conversion to the disubstituted product, which afforded the target compound in good yield (70\%). The identity of these compounds was confirmed by mass spectrometry, IR spectroscopy, ${ }^{1} \mathrm{H}$ and ${ }^{13} \mathrm{C}$ NMR spectroscopies. 


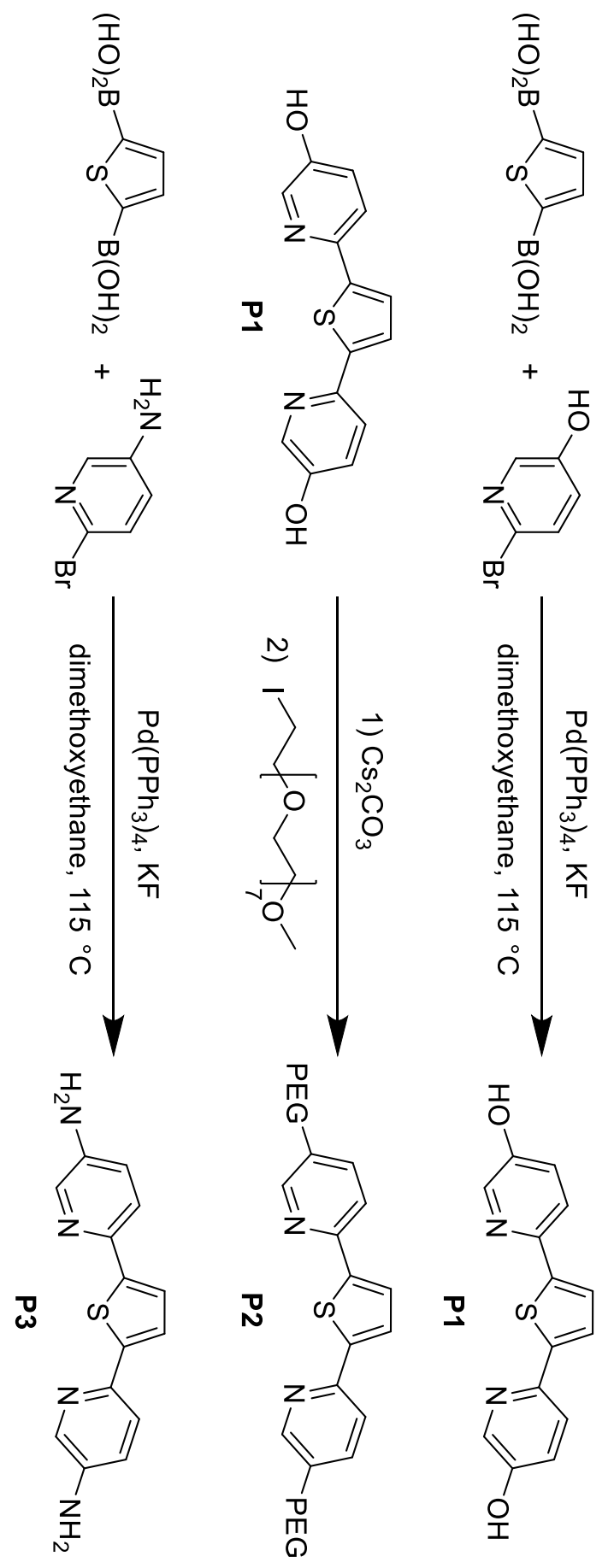

Scheme 6: Synthetic scheme of the synthesis of P1-P3. 


\subsubsection{Photophysical Properties of Pyridine-Functionalized Ligands in $\mathrm{CH}_{3} \mathrm{CN}$}

The absorption and emission of all ligands were obtained at $1 \times 10^{-5} \mathrm{M}$ concentration in $\mathrm{CH}_{3} \mathrm{CN}$ (Figure 20). The photophysics of the functionalized sensors are summarized in Table 5. The red-shift of the absorption and emission spectrum of the compounds relative to the nonfunctionalized compound (L2) is likely due to the lone pairs on the water-solubilizing functional groups of P1-P3, that are directly involved in the $\pi$-system of the aromatic compounds. ${ }^{41}$ Both $\mathbf{P 1}$ and $\mathbf{P 2}$ have similar photophysical properties likely due to the similarity of the alcohol and octaethylene glycol monomethyl ether functional groups. The oxygen-based functional groups cause a decrease in the molar absorptivity of the compounds relative to $\mathbf{L 2}$, while the nitrogen-based group of $\mathbf{P 3}$ causes an increase in the molar absorptivity.

The most notable change in the photophysics upon functionalization is a dramatic decrease in the fluorescent quantum yields $(\phi)$ of P1-P3. We believe the decrease in quantum yield is due to an increase in the number of nonradiative decay pathways, caused by a greater number of vibrational modes relative to $\mathbf{L 2}$. P2 $(\phi=0.15)$ has a greater number of vibrational modes however it retains a higher quantum yield then P1 $(\phi=0.10)$. This may be explained by the fact that the proton of the alcohol functional group of $\mathbf{P} 1$ could dissociate upon excitation resulting in a lower quantum yield. 
Table 5: Summary of photophysical data of L2, and P1-P3 acquired at $1 \times 10^{-5} \mathrm{M}$ in $\mathrm{CH}_{3} \mathrm{CN}$.

\begin{tabular}{|c|c|c|c|c|c|}
\hline & $\lambda_{\text {abs }}(\mathbf{n m})$ & $\varepsilon \times 10^{4}\left(\mathrm{M}^{-1} \cdot \mathrm{cm}^{-1}\right)$ & $\lambda_{\text {ex }}(\mathbf{n m})$ & $\lambda_{\mathrm{em}}(\mathrm{nm})$ & $\phi(\%)$ \\
\hline L2 & 342 & 2.90 & 343 & 394 & 56 \\
\hline $\mathbf{P 1}$ & 364 & 2.27 & 347 & 425 & 10 \\
\hline $\mathbf{P 2}$ & 353 & 2.09 & 336 & 410 & 15 \\
\hline P3 & 368 & 3.15 & 371 & 460 & 23 \\
\hline
\end{tabular}
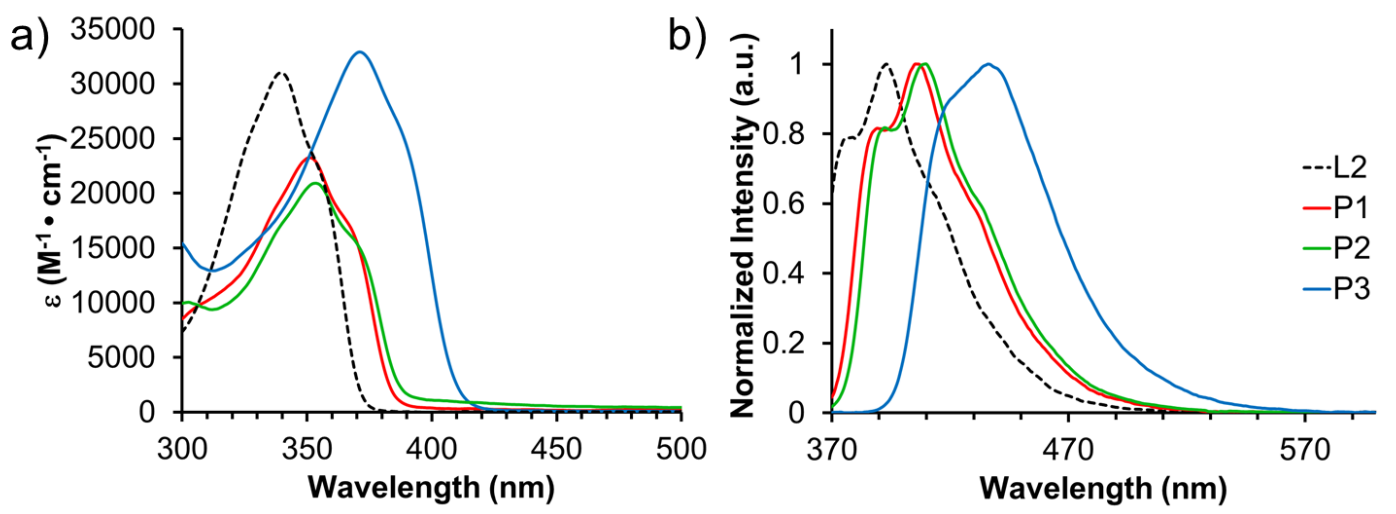

Figure 20: Absorption (a) and emission (b) spectrum of $\mathbf{L 2}$ and P1-P3 in $\mathrm{CH}_{3} \mathrm{CN}$ at $1 \times 10^{-5} \mathrm{M}$. 


\subsubsection{Photophysical Properties of Pyridine-Functionalized Ligands in Mixed Aqueous}

Media

Photophysical properties for P1-P3 have also been obtained in $\mathrm{H}_{2} \mathrm{O}$ at $1 \times 10^{-5} \mathrm{M}$ concentration (Figure 21). Notably fluorescent quantum yields for all ligands decrease in $\mathrm{H}_{2} \mathrm{O}$ relative to those obtained in $\mathrm{CH}_{3} \mathrm{CN},(\mathbf{P 1}, \phi=0.06 ; \mathbf{P} 2, \phi=0.02 ; \mathbf{P 3}, \phi=0.09)$. The drop in quantum yield could be due to water quenching the fluorescence from hydrogenbonding effects with the functional groups of the sensors. The absorption spectrum of P1$\mathbf{P 3}$ does not change in $\mathrm{H}_{2} \mathrm{O}$. Also, no changes in the emission spectrum for $\mathbf{P 1}$ and $\mathbf{P 3}$ are observed. However a $10 \mathrm{~nm}$ bathochromic shift relative to organic solvent is observed for the emission spectrum of $\mathbf{P 2}\left(\lambda_{\max }=420 \mathrm{~nm}\right)$. This agrees with the TD-DFT calculations, which suggest the transitions are $\pi$ to $\pi^{*}$ in nature and do not involve a charge transfer.
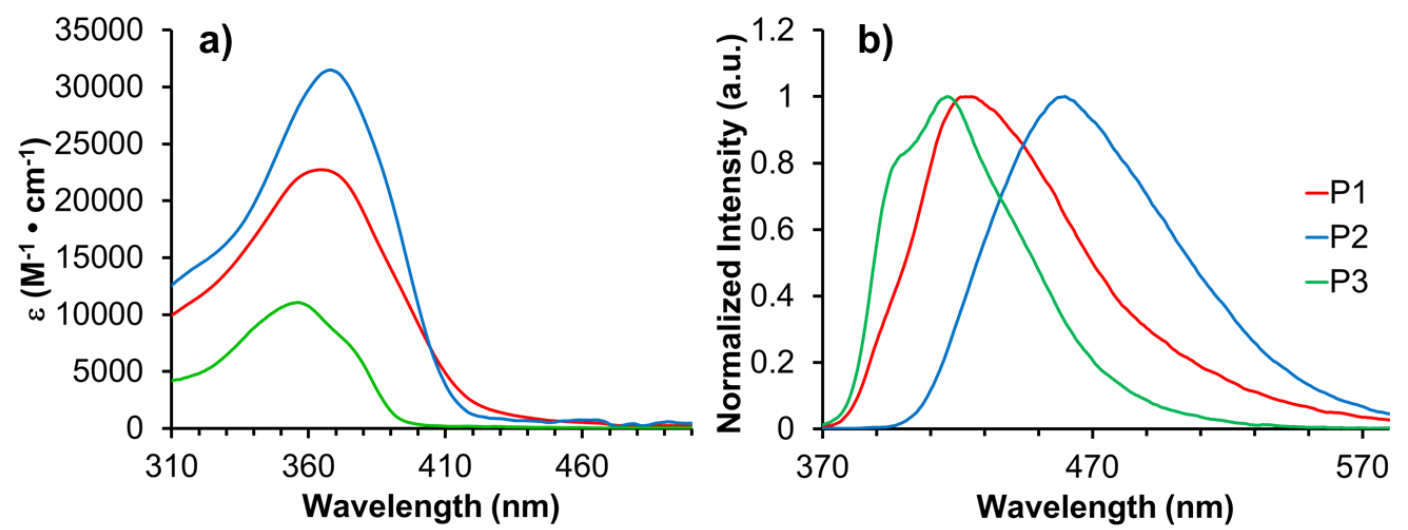

Figure 21: Absorption (a) and emission (b) of P1-P3 in $\mathrm{H}_{2} \mathrm{O}$ at $1 \times 10^{-5} \mathrm{M}$ concentration $(\mathrm{pH} 7)$.

Since the functional groups are directly involved in the aromatic system, we expect protonation or deprotonation of these groups to have an effect on the photophysical 
properties. ${ }^{41}$ Furthermore, in acidic conditions, the pyridyl nitrogen can also be protontated. In acidic conditions the $\lambda_{\max }$ of the absorption spectrum of $\mathbf{P 2}$ and $\mathbf{P 3}$ redshifts (Figure 22c \& e). No change in the $\lambda_{\max }$ of $\mathbf{P 1}$ is observed in acidic conditions. However, under basic conditions the absorption spectrum of $\mathbf{P 1}$ displays a red-shifted $\lambda_{\max }$ (Figure 22a). The same red-shift upon protonation of $\mathbf{P 2}$ and deprotonation of $\mathbf{P 1}$ is observed in the emission spectrum (Figure $22 b \& d$ ). Protonation of the amino group of P3 causes a quenching of the compound emission (Figure 22f). Quenching of the emission is likely due to excited state proton transfer of the protonated amine group. 

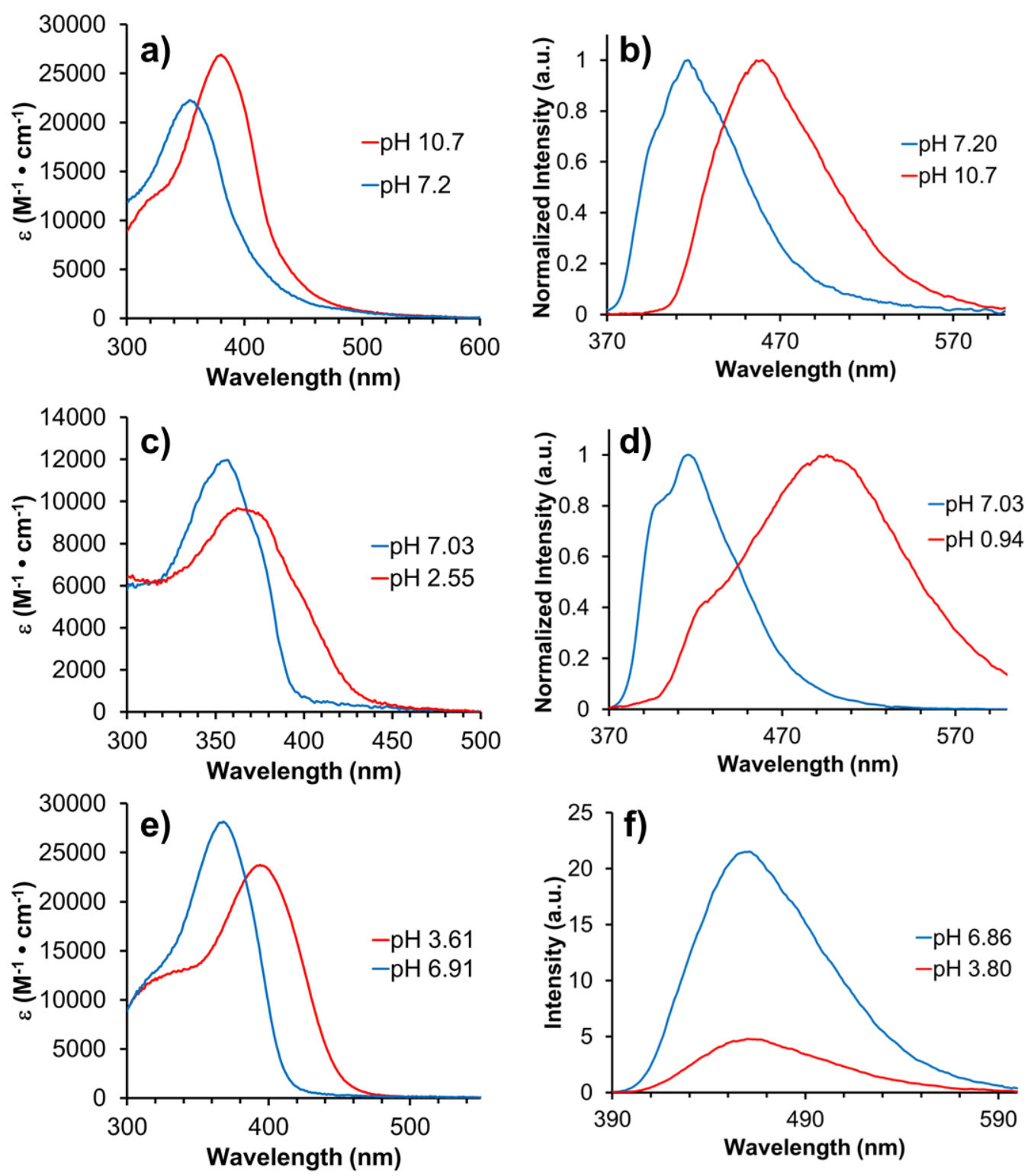

Figure 22: Absorption of P1 (a), P2 (c), and P3 (e) and emission of P1 (b), P2 (d), and $\mathbf{P 3}$ (f) in acidic (red), basic (red) or neutral (blue) conditions. Emission spectra are normalized except for $\mathbf{P 3}$ to highlight quenching.

\subsubsection{Interaction of Pyridine-Functionalized Ligands with Mercury(II) in $\mathrm{CH}_{3} \mathrm{CN}$}

The sensors for this project were designed based on $\mathbf{L 2}$, as this compound was observed to form two products in solution with increasing amounts of mercuy(II). As 
previously discussed $\mathbf{L} 2$ formed two complexes at a 2:1 and 1:1 stoichiometric ratio of sensor to metal. Therefore I believe these functionalized sensors should form complexes at similar stoichiometric ratios. These products were distinguished by the $\lambda_{\max }$ of the absorption or emission of the resulting complex..$^{83}$ The formation of two distinct products could allow for quantification of a larger dynamic range of mercury(II) concentrations, prompting the development of the pyridine functionalized sensors described in this work. The interaction of P1-P3 with mercury(II) was observed in both organic solvent $\left(\mathrm{CH}_{3} \mathrm{CN}\right)$ and mixed aqueous media $\left(50: 50 \mathrm{CH}_{3} \mathrm{CN}: \mathrm{H}_{2} \mathrm{O}\right)$.

The addition of functional groups should offer two improvements from the previous sensor design. The first improvement was to enhance the water solubility in order to be more applicable for real-world samples. In addition to water solubility the addition of these groups should also result in a greater $K_{\mathrm{a}}$ of the sensors relative to $\mathbf{L 2}$. Since the functional groups are electron-donating groups the pyridyl rings will have a greater electron density relative to $\mathbf{L} 2$. This increase in electron density should enhance the affinity of these sensors for the mercury(II) cation. The enhanced affinity will translate to a lower amount of mercury(II) required to form a detectable amount of the metal complex.

A ratiometric absorbance response to mercury(II) in $\mathrm{CH}_{3} \mathrm{CN}$ is observed for P1P3 (Figure 23). Increasing amounts of mercury(II) cause a decrease in the peak intensity of the sensor as a new red-shifted peak grows in. For all sensors addition of mercury(II) results in a colored solution allowing for ease of detection by visible inspection. Similar to $\mathbf{L 2}$, no isosbestic point is observed during the absorption titration of $\mathbf{P 2}$ suggesting the 
formation of multiple products with the addition of mercury(II). An isosbestic point is observed at low concentrations of mercury during the titration of $\mathbf{P 1}$ and $\mathbf{P 3}$ at $373 \mathrm{~nm}$ and $395 \mathrm{~nm}$ respectively.

The first product formed for P1 and $\mathbf{P 3}$ have absorbance values at $385 \mathrm{~nm}$ and 416 $\mathrm{nm}$ respectively, while the second products have absorbances at $373 \mathrm{~nm}$ and $406 \mathrm{~nm}$ respectively. Isosbestic points are only observed at relatively low mercury(II) concentrations during formation of the first product. Addition of greater than 6.00 and 0.6 eq of mercury(II) to solutions of $\mathbf{P 1}$ and $\mathbf{P 3}$, respectively, results in the disappearance of the isosbestic point as the second product is formed. The first product formed with $\mathbf{P 2}$ has an absorbance value at $382 \mathrm{~nm}$, while the second product has an absorbance at 377 nm.

The change in the emission spectrum of both $\mathbf{P 1}$ and $\mathbf{P 2}$ is similar to the absorbance response of these sensors. Addition of mercury(II) to a solution of either P1 or P2 in $\mathrm{CH}_{3} \mathrm{CN}$ causes a red-shift in the emission spectrum (Figure 24). A second emissive product is observed to form during the titration of $\mathbf{P 1}$ and $\mathbf{P 2}$ after the addition of 10.0 eq of mercury(II). These products have blue-shifted emission relative to the first product formed (Figure 24b, d, \& f). 

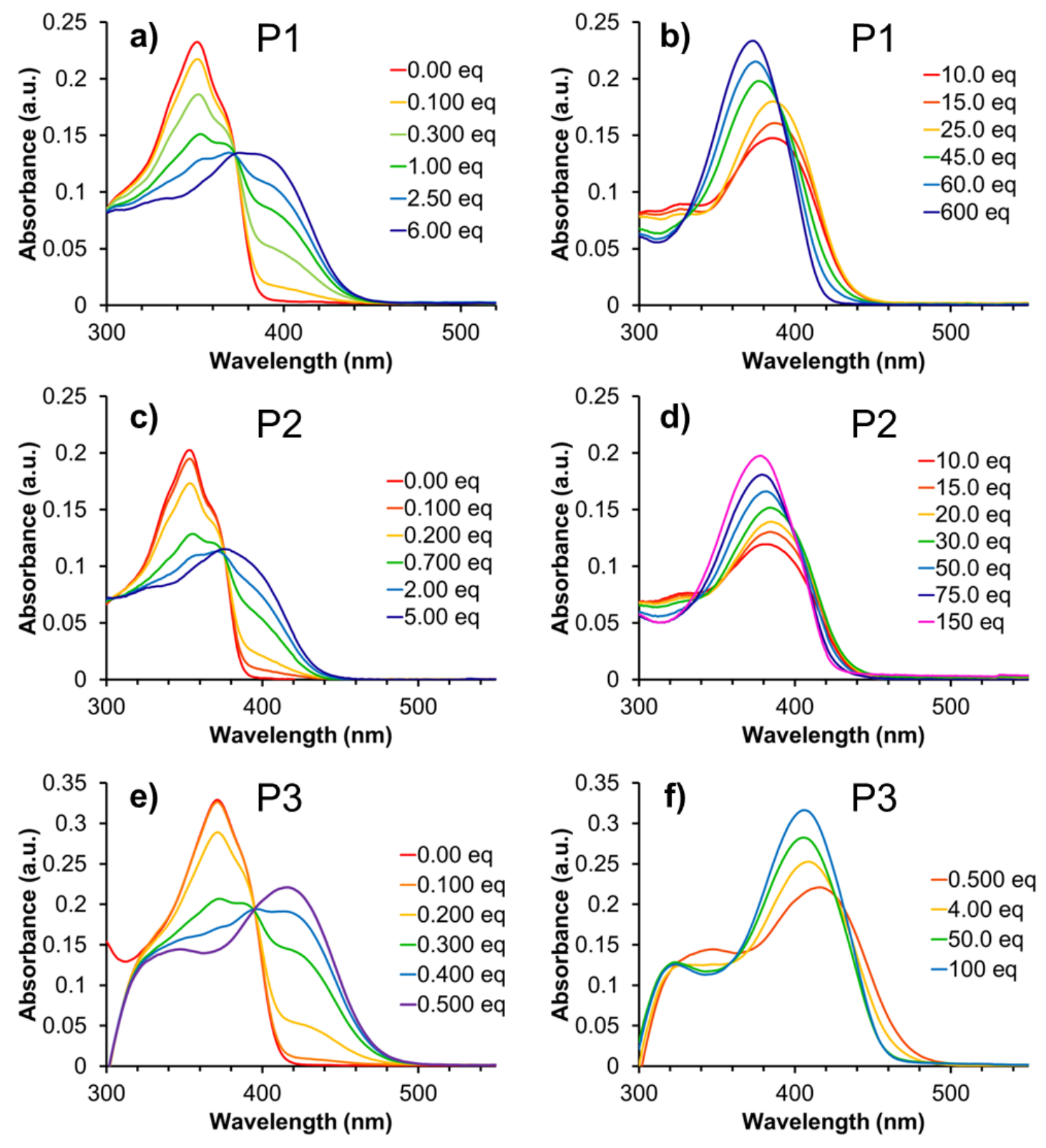

Figure 23: Change in absorption spectrum in $\mathrm{CH}_{3} \mathrm{CN}$ of $\mathbf{P 1}$ (a-b), $\mathbf{P 2}$ (c-d), and P3 (e-f) at $1 \times 10^{-5} \mathrm{M}$ with increasing amounts of $\mathrm{Hg}\left(\mathrm{ClO}_{4}\right)_{2}$. Spectra show formation of two distinct products in solution.

Compound $\mathbf{P 3}$ has a unique change in emission where addition of mercury(II) causes quenching of the ligand emission at $460 \mathrm{~nm}$ until 0.4 eq have been added (Figure 24e). Increasing amounts of mercury(II) past 0.4 eq causes a new peak to grow in at 
$483 \mathrm{~nm}$ (Figure 24f). Interestingly $\mathbf{P 3}$ has a similar initial photophysical response to mercury(II) as it does to protonation of the amine group. This suggests the first complex formed in solution with $\mathbf{P 3}$ is due to coordination of the amine group to mercury(II), that is due to the affinity of mercury(II) for amino nitrogen donor atoms. ${ }^{8,89,90}$
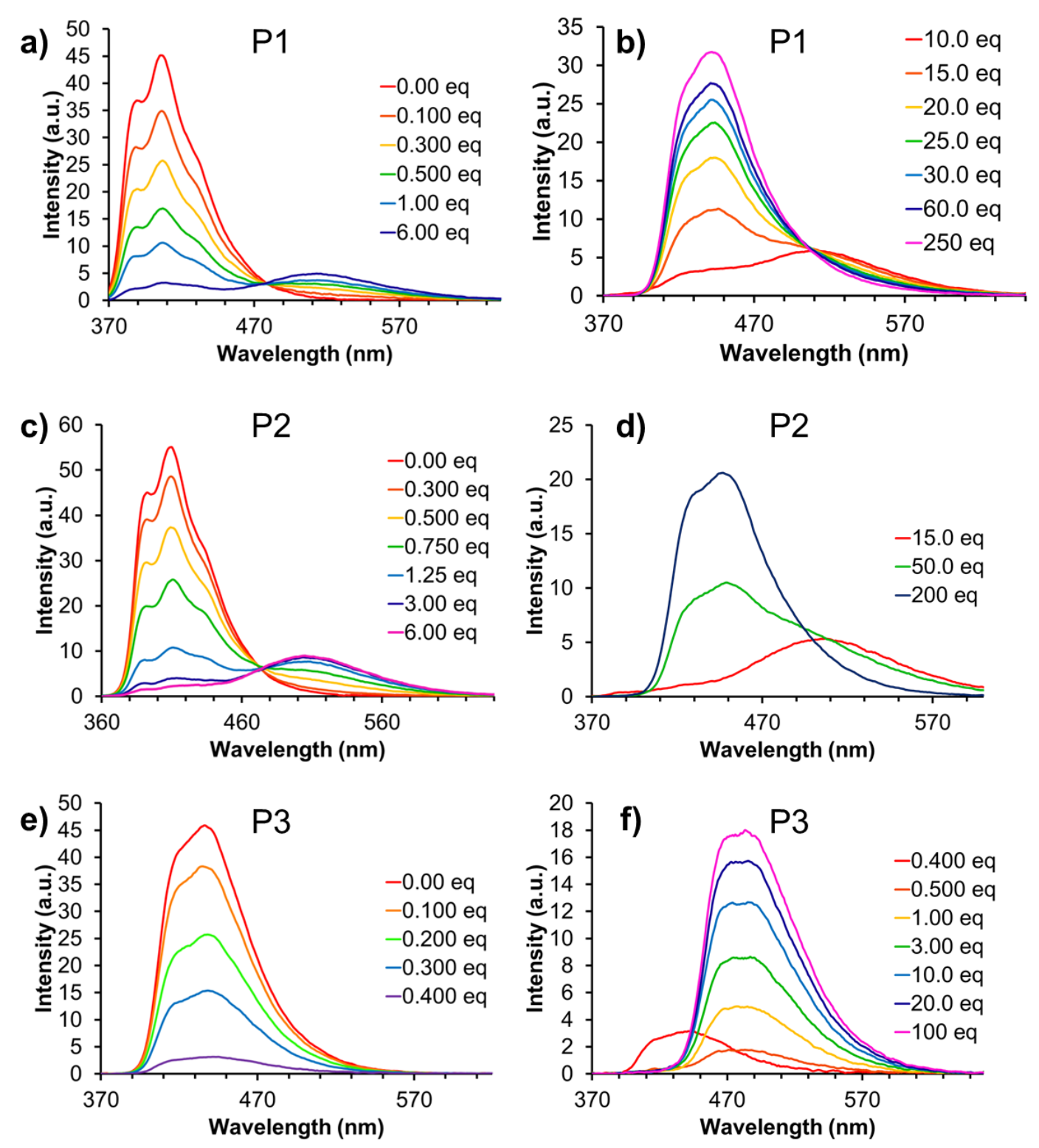

Figure 24: Change in emission spectrum in $\mathrm{CH}_{3} \mathrm{CN}$ of $\mathbf{P 1}$ (a-b), $\mathbf{P 2}$ (c-d), and $\mathbf{P 3}$ (e-f) at $1 \times 10^{-5} \mathrm{M}$ with increasing amounts of $\mathrm{Hg}\left(\mathrm{ClO}_{4}\right)_{2}$. Spectra show formation of two distinct 74 
products in solution. Titrations for solutions of P1, P2 and P3 were excited at $360 \mathrm{~nm}$, $350 \mathrm{~nm}$, and $360 \mathrm{~nm}$, respectively.

The change in the photophysical properties is attributed to coordination of the donor atoms of P1-P3 to mercury(II). Addition of EDTA to a solution of P1-P3 with excess mercury(II) results in regeneration of the original absorbance spectrum of the compounds indicating reversible binding. However, of the sensors only $\mathbf{P 2}$ has a completely reversible photo-physical response to mercury(II) since the addition of EDTA results in complete recovery of the absorption spectra of P2. The addition of EDTA does not completely return the original peak intensity for solutions of P1 and P3 with mercury(II) (Figure 25). Given the absence of the absorbance peak for the mercury complex this suggests that a small amount of $\mathbf{P 1}$ and $\mathbf{P 3}$ reacts irreversibly with mercury(II) to form new compounds, though these are not the major products formed.
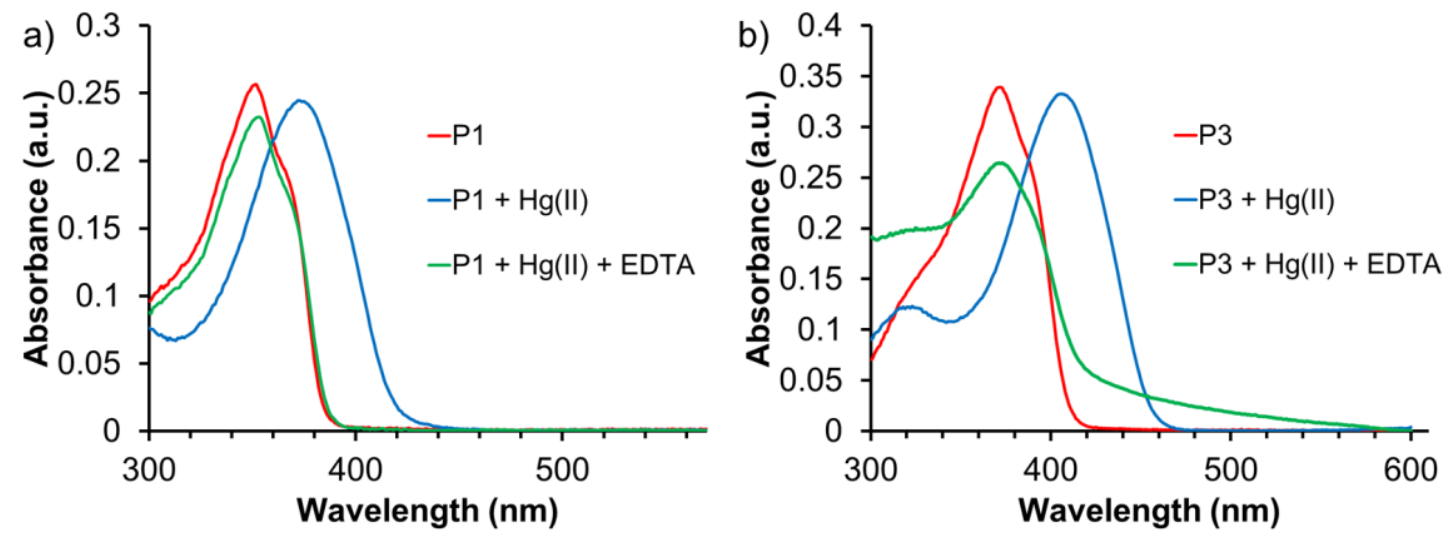

Figure 25: Change in absorption spectrum of (a) $\mathbf{P 1}$ and (b) $\mathbf{P 3}$ after addition of $\mathrm{Hg}\left(\mathrm{ClO}_{4}\right)_{2}$ (blue) and subsequent addition of EDTA (green) in $\mathrm{CH}_{3} \mathrm{CN}$ at $1 \times 10^{-5} \mathrm{M}$.

To determine the binding constants of these sensors Job's plots were obtained to estimate the binding stoichiometry (Figure 26). In addition to Job's plots, absorption 
titration data was fit to models for different ratios of sensor:mercury (1:1, 2:1 and 1:2) to determine the stoichiometry of the complexes formed with each sensor using Dr. Thordarson's fitting program in the same manner described in Chapter 3. ${ }^{79}$ Since multiple products are formed in solution data obtained from the Job's plots was difficult to interpret. P2 did not indicate any specific stoichiometry from the resulting Job's plot, which could be due to formation of both products in solution at equilibrium. Since P1 and P3 were both observed to have isosbestic points during the titrations with mercury(II) the Job's plots obtained are slightly more resolved. The Job's plot of P1 and P3 suggests that one of the formed complexes has a 1:1 and 3:2 binding stoichiometry (sensor: $\mathrm{Hg}$ ), respectively. 

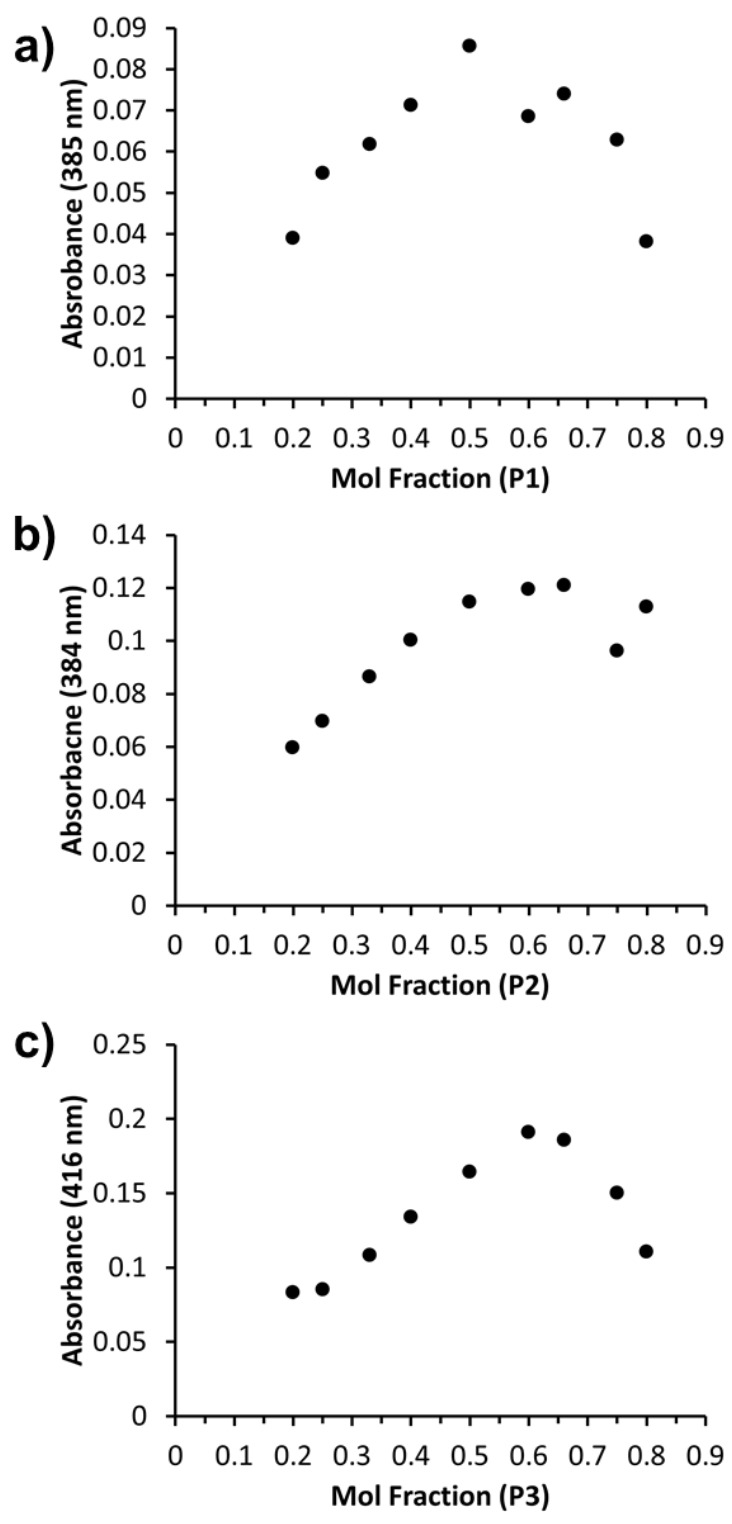

Figure 26: Job's plots obtained for (a) P1, (b) $\mathbf{P 2}$ and (c) $\mathbf{P 3}$ with mercury(II) at $1 \times 10^{-5}$ $\mathrm{M}$ in $\mathrm{CH}_{3} \mathrm{CN}$.

According to our fitting analysis, sensors L2, P1 and P2 form complexes of 2:1 (sensor:Hg) stoichiometry at low concentrations of mercury(II) followed by subsequent formation of a 1:1 complex. Initial formation of the 2:1 complex in these cases is reasonable considering the high concentration of our sensors relative to mercury(II) at the 
start of the titration. Though the Job's plot of P3 suggests a 3:2 binding stoichiometry, the titration data did not fit to a 3:2 model or any other model that was attempted $(1: 1$, 2:1, and 1:2). Therefore we were not able to obtain a binding constant for compound $\mathbf{P 3}$.

As expected, the electron donating groups causes compounds P1-P3 to be more sensitive to mercury(II) compared to unsubstituted L2. Compounds P1 and P2 have higher overall $K_{\mathrm{a}}$ of $4.52 \times 10^{10} \mathrm{M}^{-2}( \pm 18 \%)$ and $1.43 \times 10^{11} \mathrm{M}^{-2}( \pm 12 \%)$ respectively compared to $\mathbf{L} \mathbf{2}\left(K_{\mathrm{a}}=5.37 \times 10^{9} \mathrm{M}^{-2} \pm 9.8 \%\right)$. These binding constants are the overall binding constants for the formation of the 2:1 complex. Stepwise binding constants for L2-P2 are reported in Table 6.

Table 6: Association constant data obtained from fits of absorption titration data. Stepwise data constants are reported along with the overall binding constant for compounds L1, P1 and P2.

\begin{tabular}{llll}
\hline & $\boldsymbol{K}_{11}\left(\mathbf{M}^{-\mathbf{1}}\right)$ & $\boldsymbol{K}_{21}\left(\mathbf{M}^{-\mathbf{1}}\right)$ & $\boldsymbol{K}_{\boldsymbol{a}}\left(\mathbf{M}^{-\mathbf{2}}\right)$ \\
\hline $\mathbf{L 2}$ & $6.93 \times 10^{1}( \pm 4 \%)$ & $7.70 \times 10^{7}( \pm 9 \%)$ & $5.37 \times 10^{9}( \pm 4 \%)$ \\
\hline P1 & $1.15 \times 10^{2}( \pm 7 \%)$ & $3.93 \times 10^{8}( \pm 17 \%)$ & $4.52 \times 10^{10}( \pm 18 \%)$ \\
\hline P2 & $1.89 \times 10^{5}( \pm 8 \%)$ & $7.57 \times 10^{5}( \pm 8 \%)$ & $1.43 \times 10^{11}( \pm 12 \%)$ \\
\hline
\end{tabular}




\subsubsection{Interaction of Pyridine-Functionalized ligands with Mercury(II) in Mixed Aqueous Media}

Ideally mercury(II) sensors would be water soluble for testing real world samples. To assess the efficacy of P1-P3 as mercury(II) sensors in water, titrations were performed in mixed aqueous media as well as organic solvent. A large excess of mercury(II) is needed in pure $\mathrm{H}_{2} \mathrm{O}$ making it difficult to completely form both products. Therefore we performed titrations in 50:50 $\mathrm{CH}_{3} \mathrm{CN}: \mathrm{H}_{2} \mathrm{O}$ as this allowed for completion of the titrations without large amounts of mercury(II) being needed. The presence of water also appears to change the sensing mechanism of $\mathbf{P 1}$ and $\mathbf{P 3}$.

There are a number of differences in the photo-physical response that occur during the titration of P1-P3 in aqueous media compared to organic solvent. Perhaps most notably, additions of mercury(II) do not cause as great of a red-shift in the absorbance spectrum of P1-P3 relative to titrations performed in $\mathrm{CH}_{3} \mathrm{CN}$. This is observed only as a shoulder in the absorption spectrum as the sensor peak is quenched by the addition of mercury, suggesting that the formed complexes have a lower molar absorptivity value relative to those formed in organic solvent (Figure 27a, c \& e). No isosbestic point is observed during the titrations of P1-P3 suggesting two or more products still form during the titration. Increasing additions of mercury(II) eventually result in the formation of an insoluble product in solution, seen as a precipitate. The emission of P1 and P3 are quenched with increasing amounts of mercury(II) in aqueous media (Figure 27b \& f). Compound $\mathbf{P 2}$ does not have a different fluorescent response to mercury(II) at low concentrations in aqueous media when compared to organic solvent. However, larger 
equivalents of mercury(II) are still needed in order to observe a photophysical response and the second emissive product is not observed.
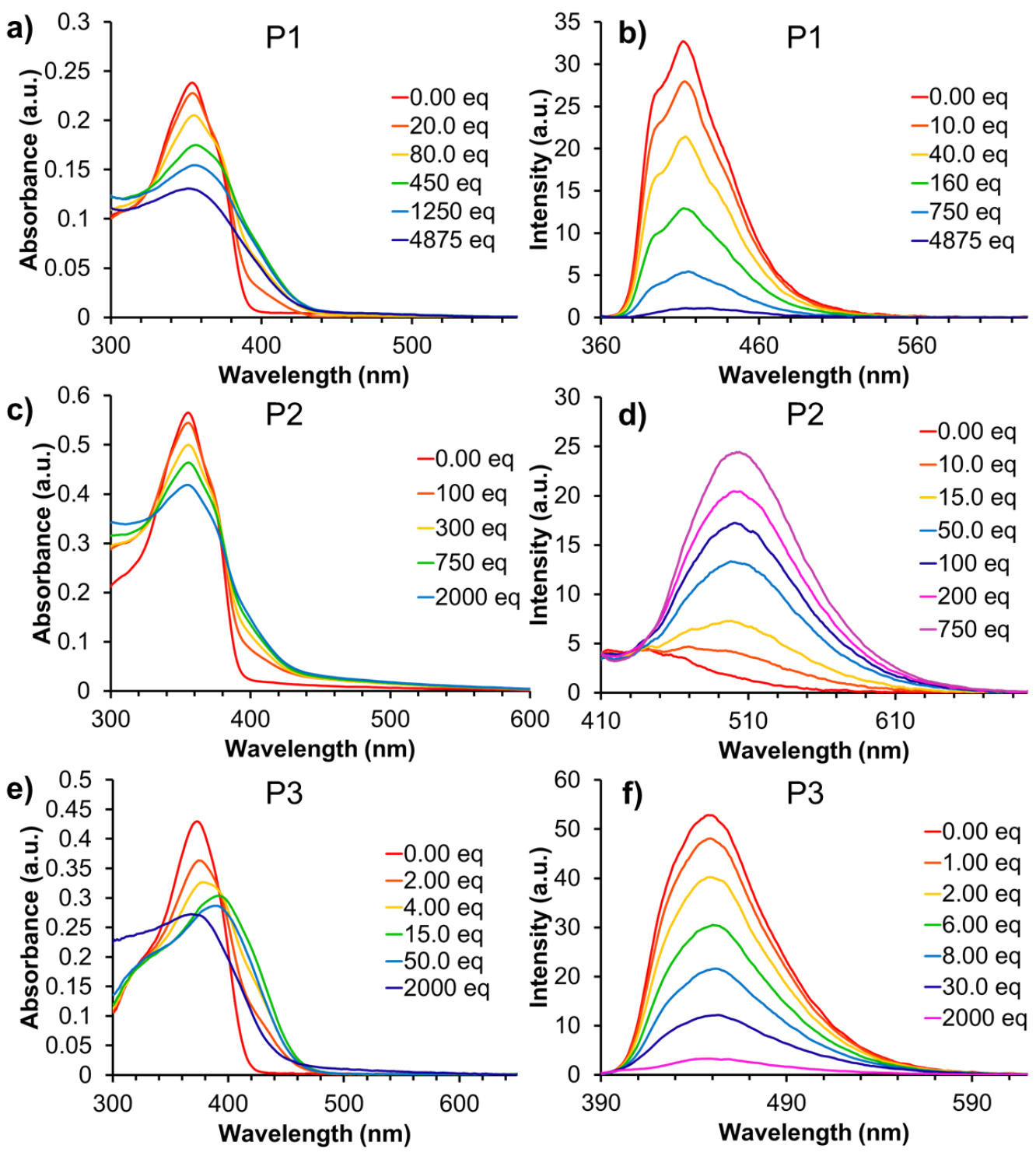

Figure 27: Absorption (a, c, \& e) and emission (b, d, \& f) titrations of P1, P2 and P3 in 50:50 $\mathrm{CH}_{3} \mathrm{CN}: \mathrm{H}_{2} \mathrm{O}$. Emission titrations are excited at and $350 \mathrm{~nm}, 400 \mathrm{~nm}$, and $360 \mathrm{~nm}$ for P1, $\mathbf{P 2}$ and $\mathbf{P 3}$ respectively. Excitation wavelengths were adjusted for solvent in order to obtain a complete spectrum and observe increasing intensity of product emission in the case of $\mathbf{P 2}$. 
Though P1-P3 are soluble in mixed aqueous media, $\mathrm{H}_{2} \mathrm{O}$ appears to greatly limit the sensitivity of the sensors for mercury(II). The photophysical response of P1-P3 is also observed to change in the presence of $\mathrm{H}_{2} \mathrm{O}$. Unlike in organic solvent, there is no visible color change in solution with increasing mercury(II) due to the lower absorptivity of the complex in mixed aqueous media. In addition, the complexes that $\mathbf{P 1}$ and $\mathbf{P 3}$ form with mercury(II) are no longer fluorescent in mixed aqueous media. Therefore $\mathbf{P 1}$ and $\mathbf{P 3}$ both act as fluorescent 'turn-off' sensors in mixed aqueous media, likely due to $\mathrm{H}_{2} \mathrm{O}$ quenching the emission of the formed complexes. Given that P1 and P3 are the only ligands whose functional groups bear hydrogen atoms this could be due intermolecular interactions between the functional groups and $\mathrm{H}_{2} \mathrm{O}$ increasing the number of nonradiative decay pathways.

\subsection{6 ${ }^{1}$ H NMR Experiments for Structure Determination}

To elucidate the structure of the complexes formed during titrations ${ }^{1} \mathrm{H}$ NMR experiments were performed in organic solvent $\left(\mathrm{CD}_{3} \mathrm{CN}\right)$ with varying concentrations of mercury(II). Downfield shifts in the spectra were attributed to deshielding of the protons due to metal coordination. ${ }^{86}$ Delocalization of the aromatic rings of the ligands results in protons para to the mercury(II) coordination site being the most deshielded and therefore

the most shifted. Similar to previous ${ }^{1} \mathrm{H}$ NMR experiments described in Chapter 3 and 4, a gradual downfield shift of the protons associated with P1-P3 is observed with increasing amounts of mercury(II) indicating exchange interactions are occurring. 

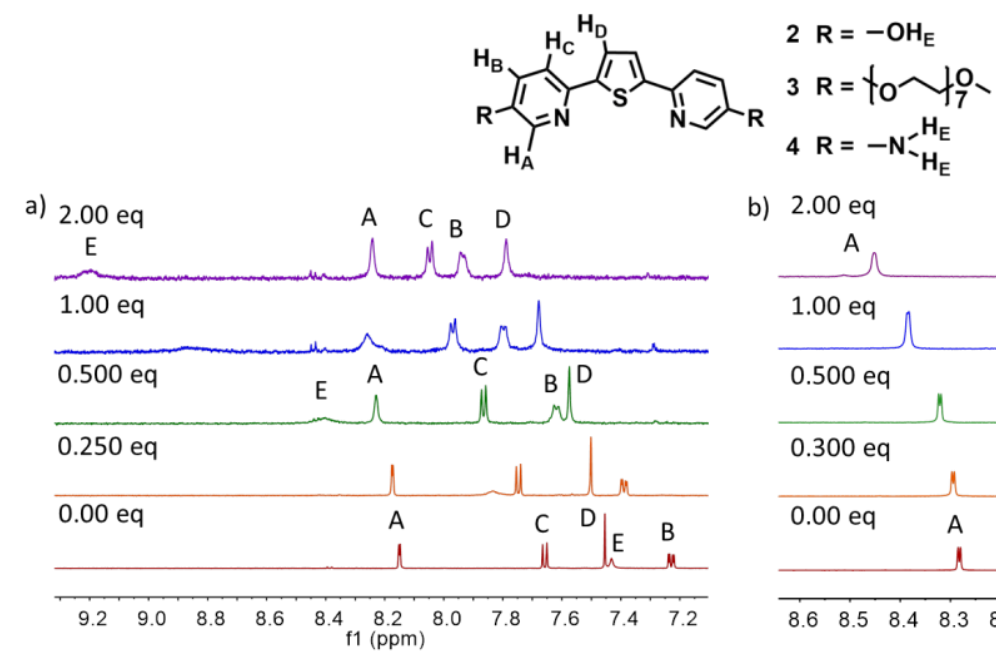

b) $2.00 \mathrm{eq}$
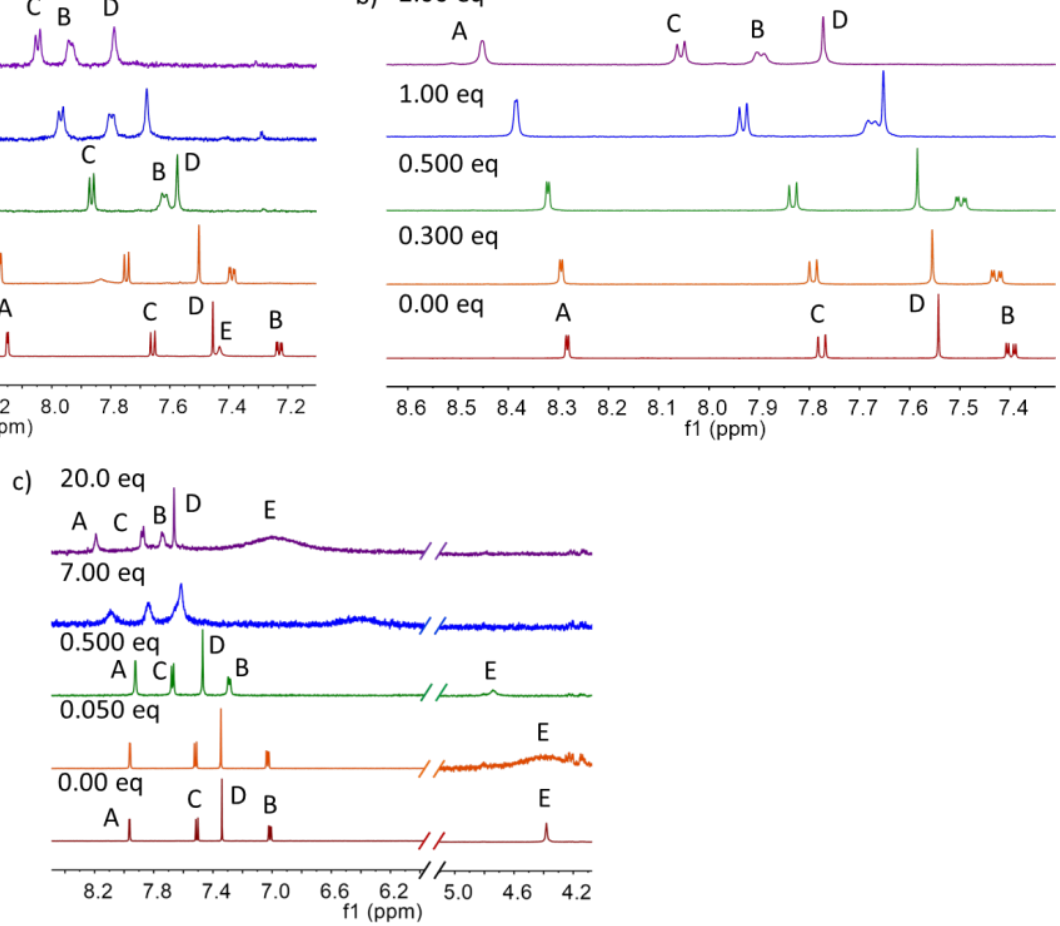

Figure 28: Change in ${ }^{1} \mathrm{H}$ NMR spectrum with increasing amounts of $\mathrm{Hg}\left(\mathrm{ClO}_{4}\right)_{2}$ for (a) P1, (b) P2, and (c) P3. A general structure for each ligand is overlaid above all three spectrum with labeled protons. The protons of the octaethylene glycol monomethyl ether chain are omitted for P2. Amine proton peaks have been intensified to make evident in (c).

A few distinctions can be noted for each of the compounds. Incremental additions of mercury(II) to a solution of $\mathbf{P 1}$ causes a downfield shift of the aromatic thienyl and pyridyl protons along with the alcohol proton $\left(\mathrm{H}_{\mathrm{E}}\right)$. These shifts suggest coordination of the thienyl sulfur, pyridyl nitrogen and the oxygen atom of the alcohol group. We were surprised to observe the relatively large downfield shift of the alcohol proton, as the alcohol oxygen is unable to chelate and therefore should not be a preferred coordination 
site. The substantial broadening of the alcohol peak suggests an exchange interaction is occurring at this site.

A gradual downfield shift of the aromatic protons of $\mathbf{P 2}$ occurs with increasing amounts of mercury(II) in solution. The downfield shifts for P2 are indicative of coordination of the pyridyl nitrogen and thienyl sulfur atoms, suggesting N,S,N-chelation. Unlike $\mathbf{P 1}$ and $\mathbf{P 3}$ the peaks associated with the functional group of $\mathbf{P 2}$ do not shift with increasing amounts of mercury(II) suggesting they are not involved in any binding interactions.

As small as 0.050 eq of mercury(II) added to a solution of $\mathbf{P 3}$ results in only the amine peak broadening. After more than 0.100 eq of mercury(II) have been added, the aromatic protons of thiophene and pyridine also begin to shift downfield. Like both P1 and $\mathbf{P 2}$ downfield shifts of the peaks associated with the pyridine and thiophene rings suggest $\mathrm{N}, \mathrm{S}, \mathrm{N}$-chelation is occurring. In addition the peak of the amino protons is observed to have the greatest downfield shift after addition of mercury(II). The interaction of the amino nitrogen and mercury(II) is likely the reason the emission of $\mathbf{P 3}$ is initially quenched with additions of mercury(II), similar to how protonation of the amino group resulted in quenching of the fluorescence of $\mathbf{P 3}$.

Given that the mercury(II) perchlorate salt is a hydrate we performed control titrations to confirm the observed downfield shifts for the alcohol and amino proton of $\mathbf{P 1}$ and P3, were due to interaction with the metal rather than exchange with $\mathrm{H}_{2} \mathrm{O}$. Increasing amounts of $\mathrm{H}_{2} \mathrm{O}$ cause a minimal downfield shift for the alcohol proton of $\mathbf{P 1}$ and no 
observable shift for the amino proton of P3. Therefore, it appears that the observed shifts of these peaks during the titration with mercury(II) can be attributed to interaction of the heteroatom of the functional group with the mercury(II) ion.

All ligands in this study appear to coordinate via the thienyl sulfur and pyridyl nitrogen atoms. There is no apparent loss in symmetry throughout any of the titrations suggesting N,S,N-chelation is occurring. The heteroatoms of the functional groups of P1 and P3 appear to be involved in exchange interactions with mercury(II) in solution as well. Given these observations and the determined stoichiometry we can begin to elucidate the structure of the formed complexes. Compounds L2, P1, and P2 appear to form both 2:1 and 1:1 complexes in solution via tridentate coordination of the pyridyl nitrogen and thienyl sulfur atoms. The 2:1 complex is formed initially due to the relatively high concentration of ligand as compared to mercury(II). Once sufficient mercury(II) has been added the 1:1 complex then becomes the preferred complex. The structure of the complexes formed with $\mathbf{P 3}$ is still unclear, though there is experimental evidence to suggest coordination events could be occurring via all heteroatoms of the sensor.

\subsubsection{Selectivity towards Mercury(II)}

The selectivity of P1-P3 for the mercury(II) ion was tested against 5 eq of other metal perchlorate salts: silver(I), cadmium(II), cobalt(II), copper(II), iron(II), iron(III), magnesium(II), manganese(II), sodium(I), nickel(II), lead(II), and zinc(II). Emission spectroscopy was used to evaluate whether a photophysical response occurred in 
response to addition of a given metal salt. For compounds P1 and P2 the emission at $444 \mathrm{~nm}$ and $507 \mathrm{~nm}$ was measured to assess whether coordination to a given metal center had occurred. Since the emission of sensor P3 was quenched at low concentrations, the $\lambda_{\max }(480 \mathrm{~nm})$ of $\mathbf{P 3}$ was monitored instead. Sensors P1-P3 are observed to have a photophysical response to copper(II), iron(II) and iron(III). Additionally sensor P3 quenches in response to lead(II) and zinc(II). In all cases competitive binding is observed with both copper(II) and iron(III), showing these sensors are in fact less selective then L2. The addition of the electron-donating functional groups has resulted in an enhanced affinity for all metal ions, and thus a loss in selectivity. Given that the compounds of this study were functionalized only on the pyridine it is likely that the location of the functional group strongly influences factors such as selectivity. 

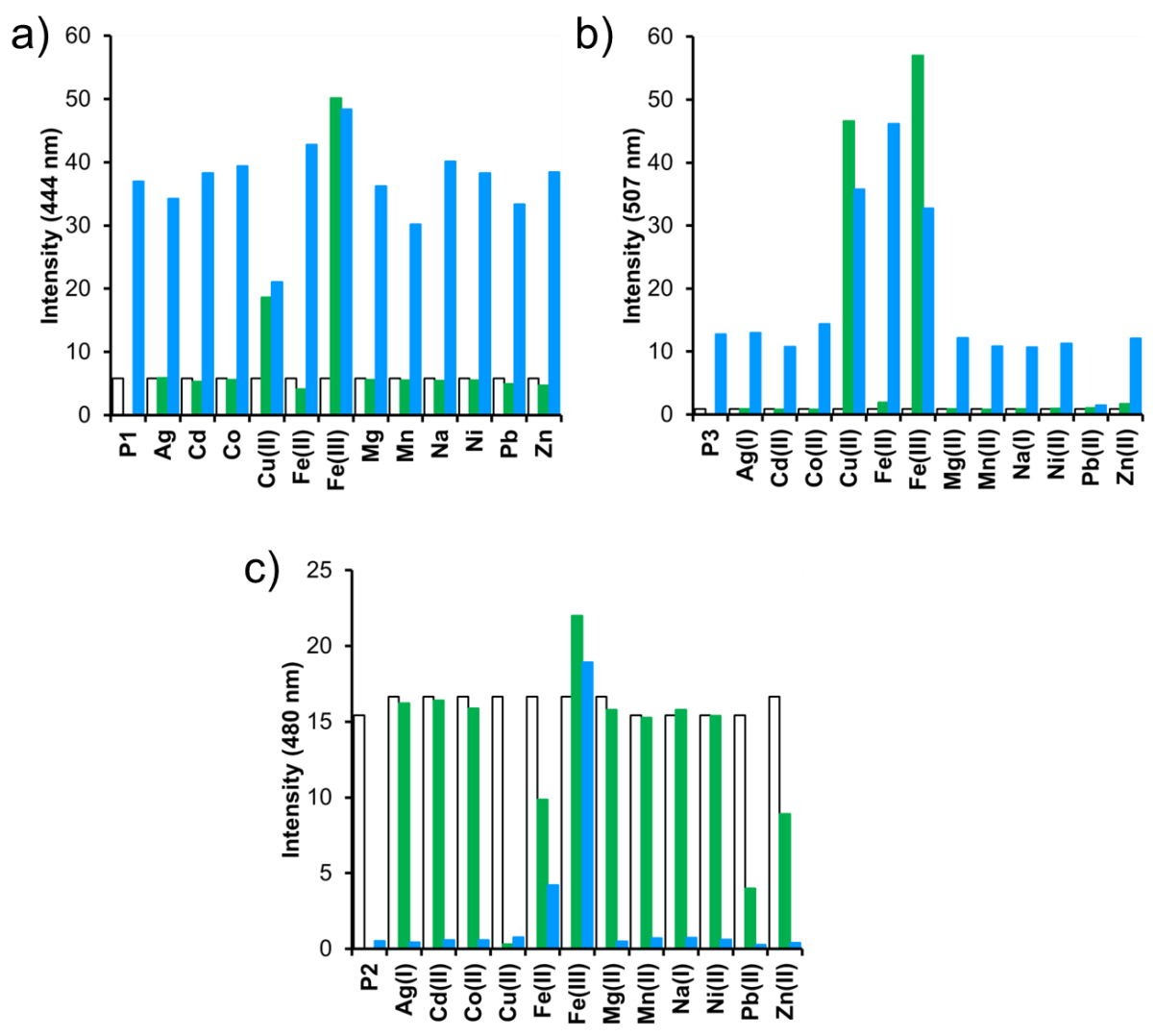

Figure 29: Emission of (a) P1, (b) P2, and (c) P3 at 444, 507, and $480 \mathrm{~nm}$, respectively, after the addition of $5 \mathrm{~mol}$ eq of various metal ions as perchlorate salts (grey bars) and then subsequent addition of $5 \mathrm{~mol}$ eq of $\mathrm{Hg}\left(\mathrm{ClO}_{4}\right)_{2}$ in $\mathrm{CH}_{3} \mathrm{CN}$. Ligand emission is indicated by white bars.

\subsection{Conclusions}

The sensors of this work were functionalized with alcohol (P1), octaethylene glycol monomethyl ether (P2) and amine (P3) groups in order to enhance water solubility for real world applications. Though these ligands have been made water soluble and have higher association constants, they exhibit less selectivity to mercury(II) in solution and do not display a clear ratiometric response in aqueous solution. The final project to conclude 
this work investigated the efficacy of functionalizing the thiophene ring of $\mathbf{L} \mathbf{2}$ to produce single-molecule multiplex sensors. 


\section{Chapter 6. Effects of Functionalization of the Thiophene Ring on Supramolecular Properties and their Potential as Single-Molecule Multiplex Sensors}

\subsection{Introduction}

Though the pyridine-functionalized ligands were shown to have an increased affinity for metal ions, this resulted in a loss of selectivity of the molecular sensors. In an attempt to better tune the selectivity for mercury(II), the thiophene ring was functionalized with electron-donating groups. The group of sensors described in this chapter were functionalized in the 3,4-positions of the thiophene ring of $\mathbf{L 2}$ with methoxy (T1), alcohol (T2), octaethyleneglycol monomethyl ether (T3) and amine (T4). Functionalization of the thiophene ring is expected to enhance the sulfur-binding affinity, translating to an increased $K_{\mathrm{a}}$ for soft Lewis acids such as mercury(II). The location of the functional groups allows for several potential coordination modes, which could be useful for the development of a single-molecule multiplex sensor to simultaneously detect multiple analytes. Furthermore, the various possible coordination modes are expected to increase the detection range by allowing the formation of several products in solution. To evaluate these sensors as multiplex detectors, the photophysical response of T1-T4 was examined with copper(II), iron(III), lead(II) and mercury(II). The effect of functionalization on the $K_{\mathrm{a}}$ and supramolecular properties will be reviewed and summarized for each metal. 


\subsection{Results}

\subsubsection{Ligand Synthesis and Structure}

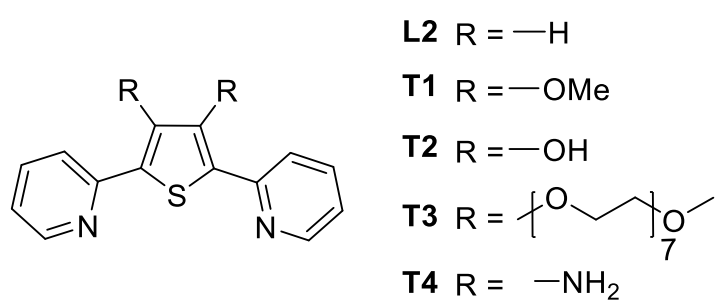

Figure 30: Structural depiction of L2, and T1-T4.

The sensors for this project were functionalized at the 3,4-position of the central thiophene ring of L2 with either methoxy (T1), alcohol (T2), octaethyleneglycol monomethyl ether (T3) or amine (T4) functional groups (Figure 30). Ancillary pyridine ligands are located at the 2,5-position of the thiophene ring and are intended to act as chelating groups with either the thienyl sulfur or the donor atoms of the functional groups. The electron-donating groups in the 3 and 4-position of the thiophene of T1-T4 are expected to enhance the thienyl sulfur binding affinity, resulting in an overall increase of the $K_{\mathrm{a}}$. This enhanced binding affinity of the thienyl sulfur should translate to an enhanced selectivity for soft Lewis acids, specifically mercury(II). The position of these groups also allows for chelation to the pyridyl nitrogen with the donor atom of the functional group (Figure 31c), as well as chelation of the functional groups alone (Figure 31b). This provides a variety of possible coordination modes containing a range of hard and soft Lewis bases and chelate-ring sizes. Depending on the metal ion certain coordination modes are expected to be preferred over others, which should provide a 
specific photophysical response. These various coordination modes are also expected to increase the number of products formed with increasing concentration of metal, from the two observed previously with $\mathbf{L} \mathbf{2}$, and consequently increase the range of detectable concentrations.

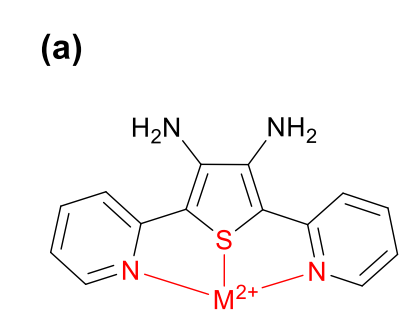

(b)
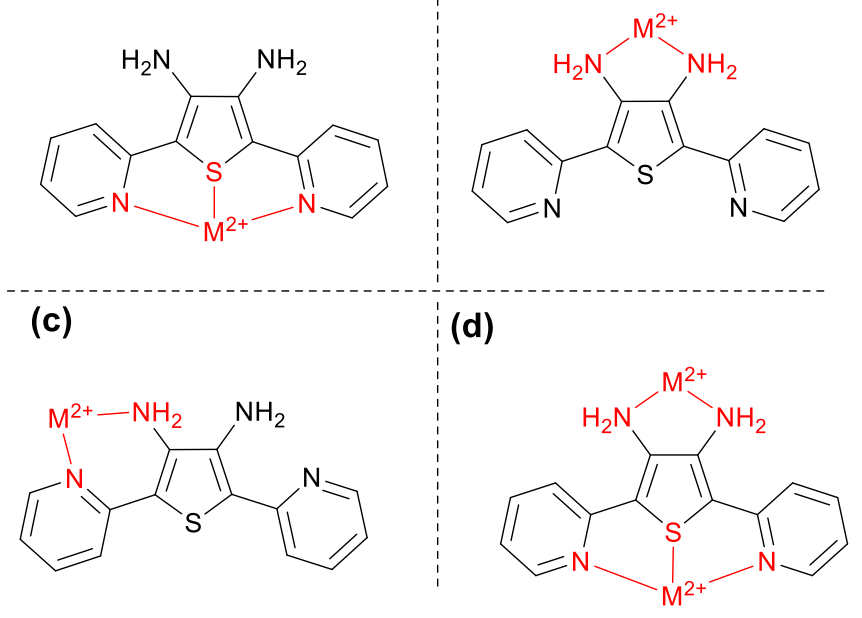

Figure 31: Examples of possible coordination complex structures with $\mathbf{T 4}$.

The synthesis of T1-T3 began with Stille cross coupling reactions to join the pyridine and thiophene rings (Scheme 7). This afforded T1 in a single step in poor yield (27.9\%). Boron tribromide was initially used to reduce the methoxy-substiutent to an alcohol group, however this was an unsuccessful attempt at synthesizing T2. Instead reduction of methoxy group of $\mathbf{T 1}$ was done with molten pyridine hydrochloride and afforded $\mathbf{T} 2$ in good yield $(68.7 \%) .{ }^{91} \mathbf{T 3}$ was synthesized from $\mathbf{T 2}$ via two separate additions of iodoheptaethyleneglycol monomethyl ether in the presence of base at elevated temperatures in good yield $(81.1 \%) .{ }^{85}$ The synthesis of $\mathbf{T 4}$ was done via a twostep procedure beginning with a Stille cross coupling to generate the nitro-substituted 
starting material in poor yield $(30.9 \%)$. Reduction of the nitro-group was completed using tin(II) chloride and afforded T4 in excellent yield (99\%). ${ }^{92}$ To the best of my knowledge, none of these compounds have been previously reported.

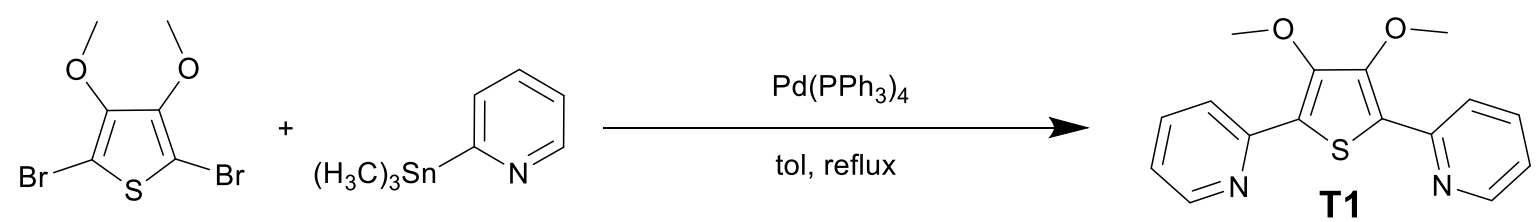

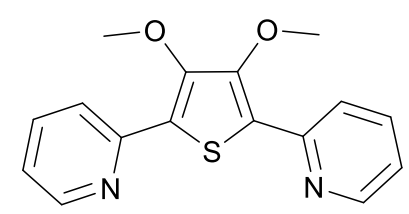

T1<smiles>Oc1c(-c2ccccn2)sc(-c2ccccn2)c1O</smiles>

T2

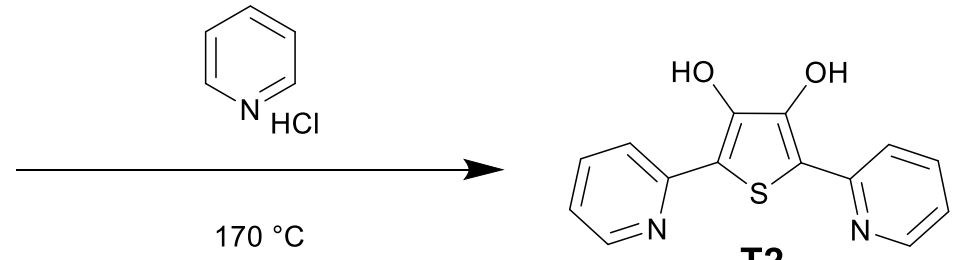

T2<smiles>CCOC(C)(C)COCCCI</smiles>

T3<smiles>O=[N+]([O-])c1c(Br)sc(Br)c1[N+](=O)[O-]</smiles><smiles>[X]C1CCCCC1</smiles><smiles>O=[N+]([O-])c1c(-c2ccccn2)sc(-c2ccccn2)c1[N+](=O)[O-]</smiles><smiles>O=[N+]([O-])c1c(-c2ccccn2)sc(-c2ccccn2)c1[N+](=O)[O-]</smiles><smiles>CCCCCC</smiles>

Scheme 7: Synthetic scheme for the synthesis of T1-T4. 


\subsubsection{Photophysical Properties of Thiophene-Functionalized Sensors}
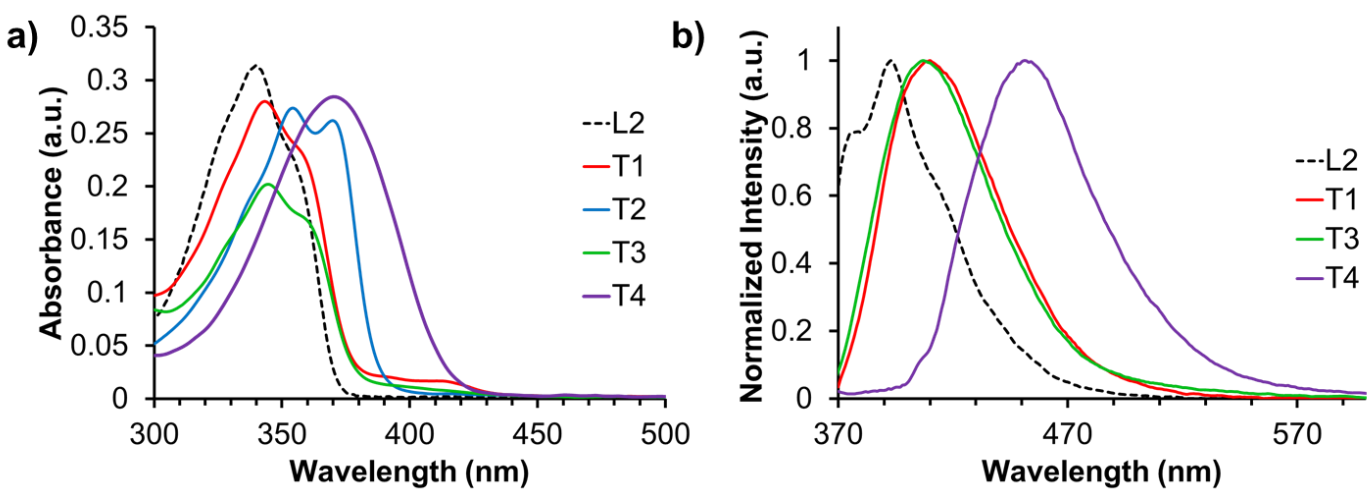

Figure 32: Absorption (a) and emission (b) of $\mathbf{L 2}$ and T1-T4 in $\mathrm{CH}_{3} \mathrm{CN}$. Solutions of $\mathbf{L 2}$, T1, T2 and T4 are at $1 \times 10^{-5} \mathrm{M}$ while $\mathbf{T 3}$ is at $2 \times 10^{-5} \mathrm{M}$.

The photophysical properties of T1-T4 have been measured in $\mathrm{CH}_{3} \mathrm{CN}$ at $1 \times 10^{-5} \mathrm{M}$ or $2 \times 10^{-5} \mathrm{M}$ (Table 7). For T3 the molar absorptivity was too low for good resolution at $1 \times 10^{-5} \mathrm{M}$, thus the concentration was increased. All ligands have redshifted absorption and emission as compared to the non-functionalized L2 (Figure 32). Functionalization of the thiophene ring has also resulted in a decrease in the molar absorptivity of all sensors studied compared to $\mathbf{L 2}\left(2.90 \times 10^{4} \mathrm{M}^{-1} \cdot \mathrm{cm}^{-1}\right)$.

Similar to the pyridine-functionalized ligands described in Chapter 5, the quantum yields $(\phi)$ of T1-T4 dropped relative to the unsubstituted sensor (L2). Generally all sensors from this study had quantum yields close to $1 \%$. The observed drop in quantum yields is likely due to an increase in the number of vibrational modes upon functionalization. Notably $\mathbf{T} 2$ is not emissive in solution. This is likely due to excited 
state proton transfer of the alcohol oxygen to the pyridyl nitrogen. The excited state proton transfer appears to be facilitated by intramolecular hydrogen bonding of the pyridyl nitrogen and alcohol proton. This is in direct contrast to P1, described in the previous chapter, where the location of the alcohol group only allowed intermolecular hydrogen bonding. Thus quenching of the emission is only observed for $\mathbf{T} 2$.

Table 7: Summary of photophysical data of T1-T4.

\begin{tabular}{cccccc}
\hline & $\boldsymbol{\lambda}_{\text {abs }}(\mathbf{n m})$ & $\boldsymbol{\varepsilon} \times \mathbf{1 0}^{\mathbf{4}}\left(\mathbf{M}^{\left.\mathbf{- 1} \cdot \mathbf{c m}^{-\mathbf{1}}\right)}\right.$ & $\boldsymbol{\lambda}_{\text {ex }}(\mathbf{n m})$ & $\boldsymbol{\lambda}_{\text {em }}(\mathbf{n m})$ & $\phi(\%)$ \\
\hline $\mathbf{T 1}$ & 343 & 2.80 & 390 & 410 & 1.4 \\
$\mathbf{T 2}$ & 354 & 2.74 & N/A & N/A & N/A \\
$\mathbf{T 3}$ & 345 & 1.01 & 350 & 407 & 2.2 \\
$\mathbf{T 4}$ & 370 & 2.84 & 360 & 451 & 1.1 \\
\hline
\end{tabular}

DFT calculations were performed to determine the lowest energy geometry, and model the electronic transitions of T1-T4. The optimized geometries of all ligands show the thiophene and pyridine rings are in plane with each other for maximum $\pi$-orbital overlap. T3 is the only sensor which is not entirely planar, due to the steric hindrance of the large functional group. Interestingly the pyridyl rings of $\mathbf{T} \mathbf{1}$ and $\mathbf{T} 3$ have rotated such that the pyridyl nitrogens are adjacent to the thienyl sulfur, unlike previous sensors (Figure 33). This arrangement of the heteroatoms in the ring should facilitate tridentate 93 
coordination of the pyridyl nitrogen and thienyl sulfur atoms. The low energy transitions are all HOMO to LUMO and $\pi$ to $\pi^{*}$ in nature. The calculated transitions appear consistent with those observed experimentally. The trend of absorption energies observed experimentally is preserved in calculations as the calculated transitions for $\mathbf{T 1}, \mathbf{T 2}, \mathbf{T 3}$ and T4 occur at $355 \mathrm{~nm}, 404 \mathrm{~nm}, 356 \mathrm{~nm}$, and $435 \mathrm{~nm}$ respectively.
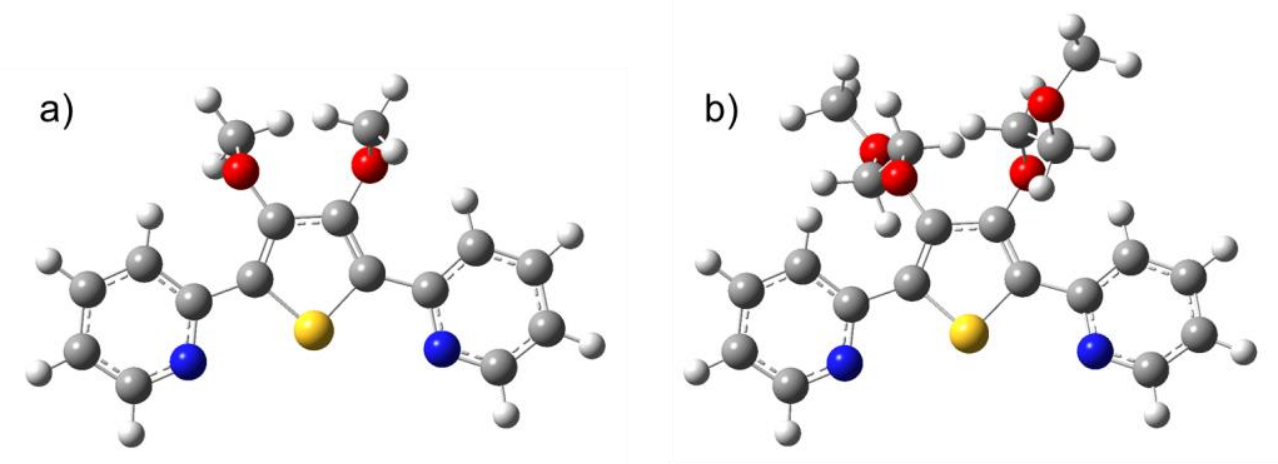

Figure 33: Optimized geometries of (a) T1 and (b) T3 determined by DFT calculations (B3LYP /6-311 + G(d)).

In Sections 6.2.3-6.2.6 I will describe the optical response of T1-T4 with copper(II), iron(III), lead(II) and mercury(II). Metal perchlorate salts were used for all titrations to maintain the solubility of the formed complexes, and for consistency. Although these ligands bear water-solubilizing functional groups, the photophysical properties and response were only examined in organic solvent. This was due to the less than ideal responses obtained for the pyridine-functionalized ligands described in Chapter 5. Instead the focus of this work is evaluating the effect functionalization of the thiophene ring has on selectivity and $K_{\mathrm{a}}$ for the mercury(II) ion, comparing the 
supramolecular properties of each sensor for various metals, and identifying potential multiplex sensors based on the observed photophysical responses.

\subsubsection{Reactivity of $\mathbf{T 1}$ with $\mathrm{Cu}^{2+}, \mathrm{Fe}^{3+}, \mathrm{Pb}^{2+}$, and $\mathrm{Hg}^{2+}$}

Of the sensors in this study $\mathbf{T 1}$ provides the most generic response to the metal ions tested. Addition of copper(II), iron(III), mercury(II) or lead(II) to a solution of T1 in $\mathrm{CH}_{3} \mathrm{CN}$ resulted in a red-shifted ratiometric response (Figure $34 \&$ Figure 35) with an identical absorption spectrum for all four metal complexes with $\lambda_{\max }$ at $385 \mathrm{~nm}$. The addition of metal salt results in a bright-yellow colored solution, allowing for ease of detection by visible inspection and could allow $\mathbf{T} 1$ to be useful as a colorimetric sensor for these metals. Further addition of iron(III), and mercury(II) salts results in a subsequent blue-shift of the absorption spectrum until saturation occurs with a large excess of the metal salt. This second product was not observed for copper(II) or lead(II). In the case of copper(II) interference in the absorption spectra from the free copper(II) salt in solution made it impossible to identify any later products. The relatively low binding affinity for lead(II) required large concentrations of metal salt to obtain the first product, and even at the 1000 eq of metal required for complete quenching of the ligand peak only the single peak was observed. 
a)

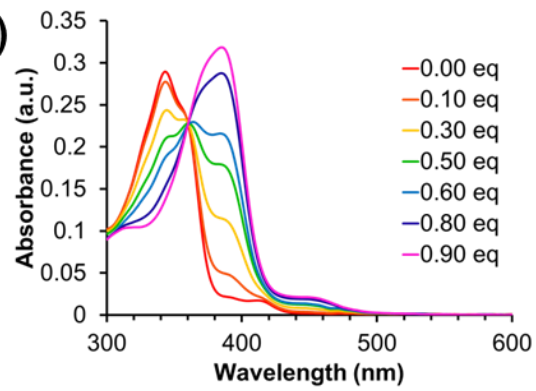

$\mathrm{Fe}^{3+}$

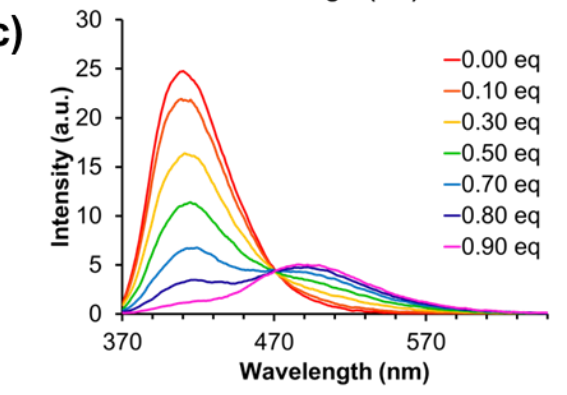

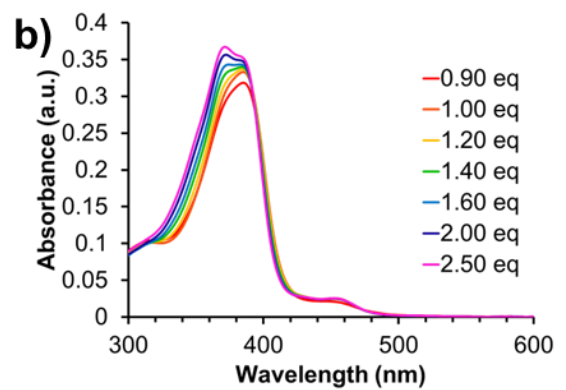

d)

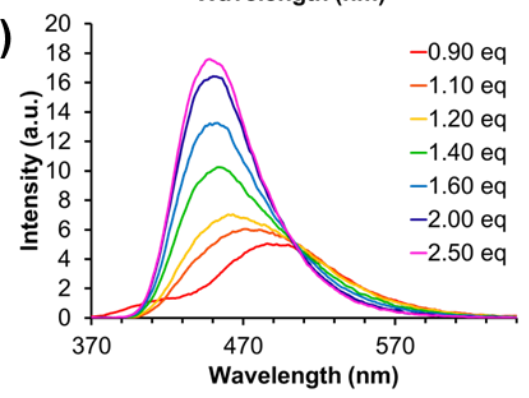

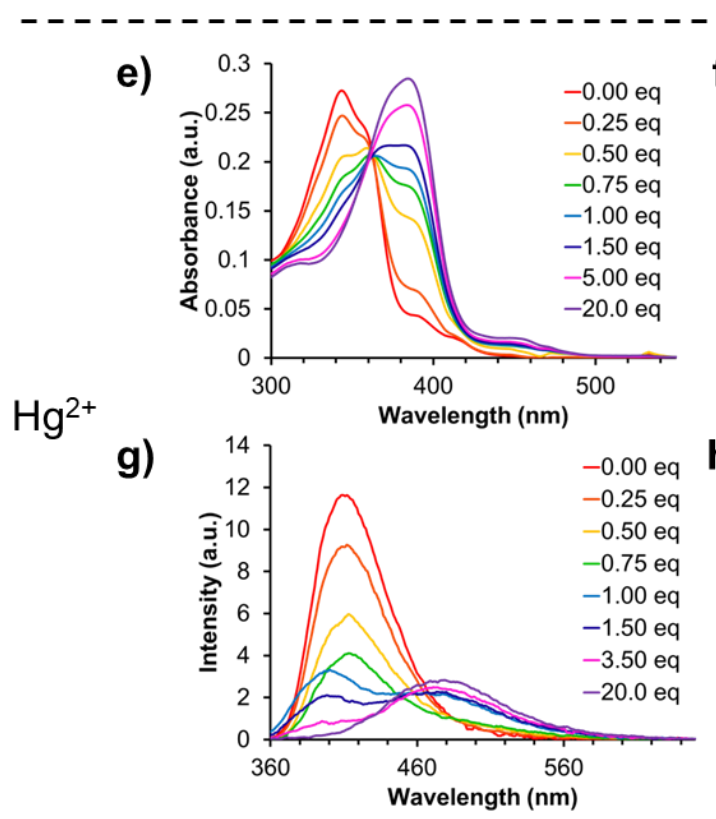

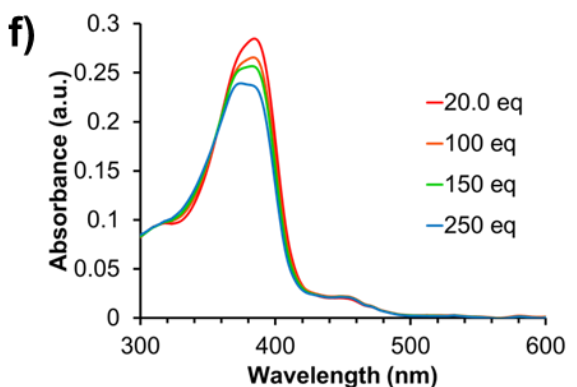

h)

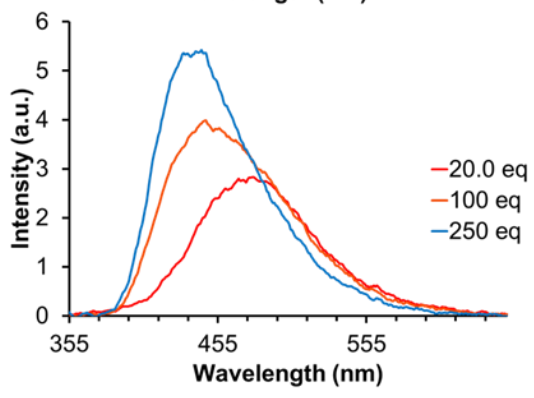

Figure 34: Absorption and emission response of T1 to iron(III) (a-d) and mercury(II) (eh) in $\mathrm{CH}_{3} \mathrm{CN}$ at $1 \times 10^{-5} \mathrm{M}$. Emission spectrum were obtained by excitation at $360 \mathrm{~nm}$ (cd) and $345 \mathrm{~nm}(\mathrm{~g}-\mathrm{h})$. 

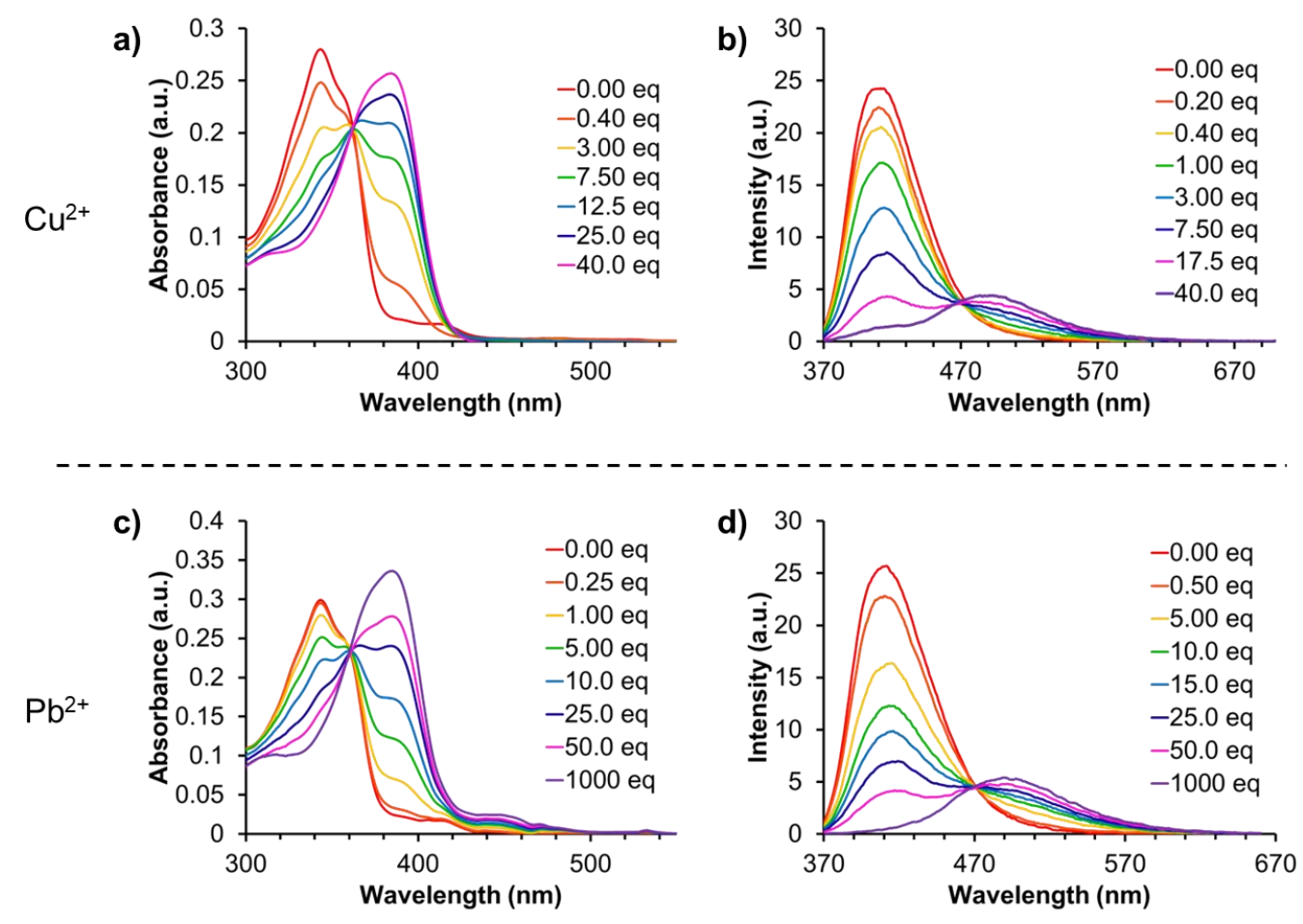

Figure 35: Absorption and emission response of T1 to copper(II) (a-b) and lead(II) (c-d) in $\mathrm{CH}_{3} \mathrm{CN}$ at $1 \times 10^{-5} \mathrm{M}$. Emission spectrum were obtained with an excitation wavelength of $360 \mathrm{~nm}$.

The emission response to copper(II), iron(III), lead(II) and mercury(II) results in a red-shifted ratiometric response mirroring the absorption response. Further addition of iron(III) or mercury(II) results in the formation of a second product with a blue-shifted emission (Figure 34). As a heavy-metal lead(II) ordinarily results in quenching of sensor emission, thus it is rare to observe a ratiometric response for this metal (Figure 35d). This unique ratiometric response to lead(II) suggests that $\mathbf{T 1}$ could be a useful model for the design of a ratiometric lead(II) sensor. 
Table 8: Association constants for copper(II), iron(III), lead(II) and mercury(II) with T1 in $\mathrm{CH}_{3} \mathrm{CN}$ obtained from absorption titrations.

\begin{tabular}{cc}
\hline Metal ion & $\boldsymbol{K}_{\mathbf{a}}$ \\
\hline Copper(II) & $3.26 \times 10^{8} \mathrm{M}^{-2}( \pm 10.7 \%)$ \\
\hline Iron(III) & $1.23 \times 10^{9} \mathrm{M}^{-2}( \pm 1.68 \%)$ \\
\hline Lead(II) & $2.10 \times 10^{7} \mathrm{M}^{-2}( \pm 8.31 \%)$ \\
Mercury(II) & $4.34 \times 10^{9} \mathrm{M}^{-2}( \pm 13.8 \%)$ \\
\hline
\end{tabular}

Association constants were obtained from absorption titration data for each metal using the Thordarson program, described previously (Table 8). ${ }^{79}$ Though the methoxy groups are electron-donating in nature, a decrease in the $K_{\mathrm{a}}$ for mercury(II) is observed as compared to $\mathbf{L 2}\left(5.37 \times 10^{9} \mathbf{M}^{-2} \pm 9.8 \%\right)$. Similar to $\mathbf{L 2}$, the obtained association constants suggest a 2:1 binding stoichiometry (T1:metal) at low concentrations of the metal salts added. Of the metals tested lead(II) has the lowest $K_{\mathrm{a}}$ followed by copper(II), while both iron(III) and mercury(II) have relatively similar values.

Based on the similar binding stoichiometries and photophysical responses I suggest a similar coordination mode is occurring with each metal. In order to confirm this ${ }^{1} \mathrm{H}$ NMR experiments were performed with each metal. For all metals N,S,N-chelation appears to occur based on the observed downfield shifts of the pyridyl peaks with 
increasing metal concentration (Figure 36), however several distinctions are noted between the addition of each metal. In the case of copper(II) both the pyridyl and methoxy peaks shift downfield and broaden until they are no longer observed, as expected due to the paramagnetic nature of the metal. Unlike lead(II) greater concentrations of iron(III) and mercury(II) results in broadening of the pyridyl and methoxy peaks, suggesting exchange interactions are occurring. Similar to L2 the two products observed for $\mathbf{T} 1$ are complexes with a 2:1 and 1:1 stoichiometry of sensor to metal with increasing metal concenetration. The source of the broadening is likely due to dissociation of a single molecule of $\mathbf{T} 1$ to form the 1:1 product. Thus once greater concentrations of metal have been added the peaks begin to sharpen, as the equilibrium is shifted such that the $1: 1$ becomes preferred. In the case of lead(II) broadening is not observed, potentially due to the low binding affinity which requires large concentrations of metal to form even one product. Though the pyridyl peaks have the greatest shift in the ${ }^{1} \mathrm{H}$ NMR spectrum, the peaks of the methoxy functional groups are observed to shift as well with addition of any metal. However these shifts are relatively minor in comparison to those of the pyridyl peaks, offering good evidence that N,S,N-chelation is indeed occurring in all cases. 


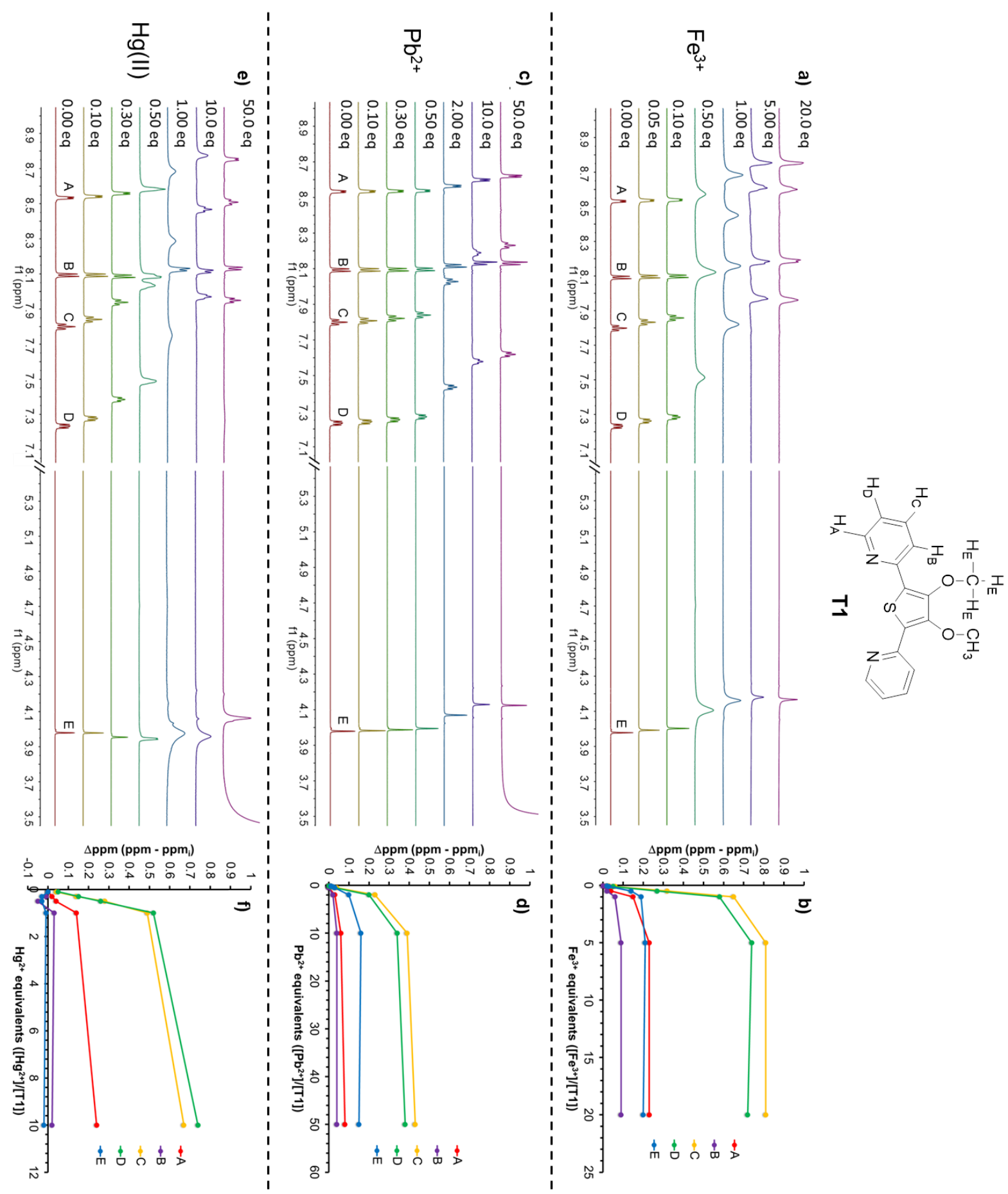

Figure 36: ${ }^{1} \mathrm{H}$ NMR titration of T1 at $4 \times 10^{-3} \mathrm{M}$ with (a) iron(III), (c) lead(II), and (e) mercury(II) with increasing amounts of metal perchlorate salt in $\mathrm{CD}_{3} \mathrm{CN}$. The magnitude of the chemical shift is plotted for each peak with increasing equivalents of (b) iron(III), (d) lead, and mercury(II). 
Since T1 did not provide specific optical responses for either copper(II), iron(III), lead(II) or mercury(II) it is not applicable as a single-molecule multiplex sensor. However competition experiments do suggest that $\mathbf{T 1}$ is selective for iron(III) against other transition metals (Figure 37). Of the metals tested, T1 is observed to have a minimal absorption response to copper(II), iron(II), mercury(II), lead(II) and zinc(II). Subsequent addition of iron(III) to these solutions appears to result in displacement of the metal ion based on the identical absorption response obtained when only iron(III) is present (Figure 37). This suggests $\mathbf{T 1}$ is selective for iron(III) and can be applicable as an iron(III) sensor.

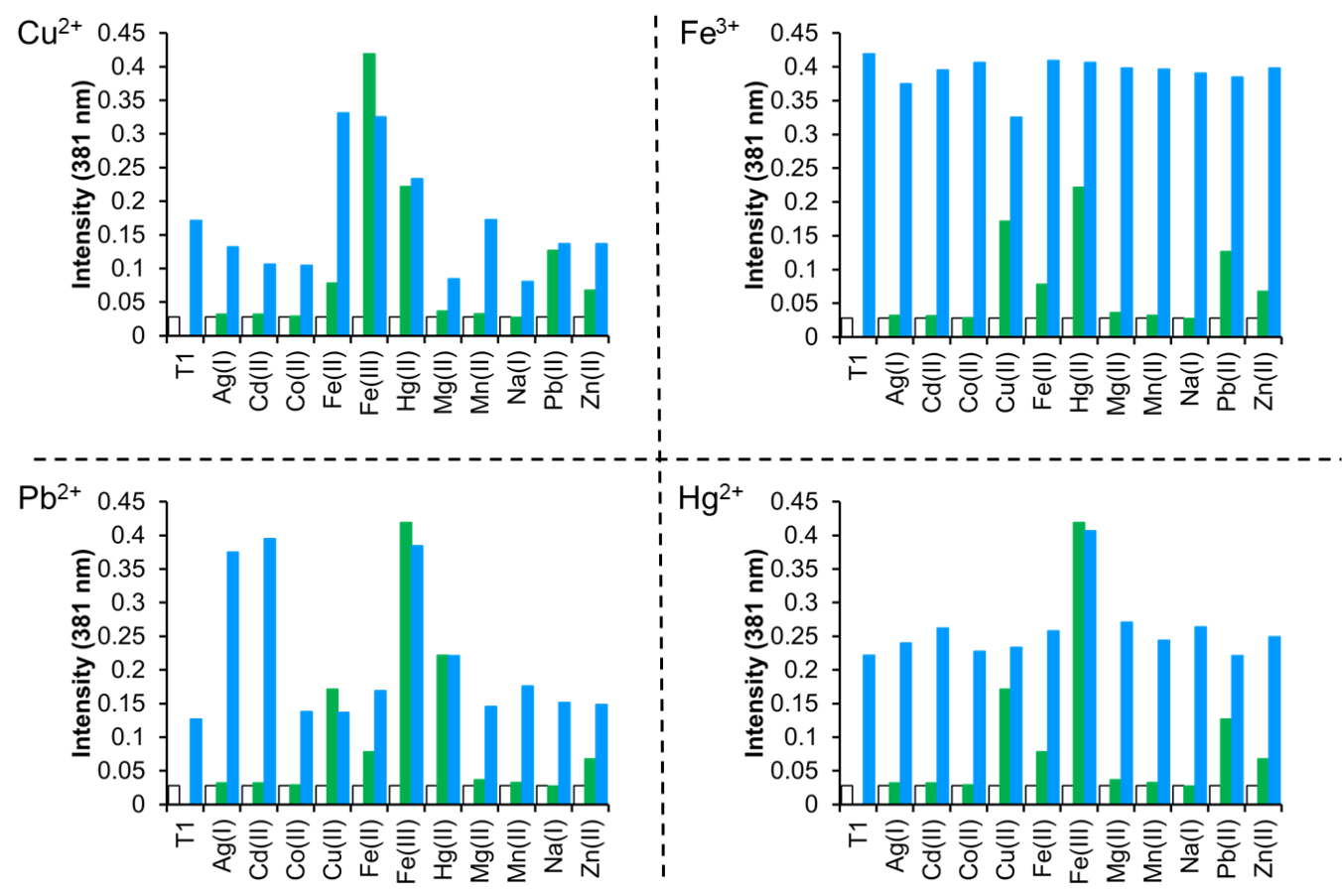

Figure 37: Absorption at the complex peak $(381 \mathrm{~nm})$ of $\mathbf{T 1}$ (white bars), after the addition of 5 eq. of metal ion (green bars), and subsequent addition of 5 eq. of either copper(II), iron(III), lead(II) or mercury(II) perchlorate (blue bars) in $\mathrm{CH}_{3} \mathrm{CN}$. 


\subsubsection{Reactivity of $\mathbf{T} 2$ to $\mathrm{Cu}^{2+}, \mathrm{Fe}^{3+}, \mathrm{Pb}^{2+}$ and $\mathrm{Hg}^{2+}$}

A number of unique features are observed during titrations of T2 with copper(II), iron(III), mercury(II) and lead(II). A red-shifted ratiometric absorbance and emission response is observed with the addition of a metal salt to $\mathbf{T} 2$, similar to previous sensors discussed in this document. Unlike previous sensors, however, three distinct products are observed to form throughout the titration with all metals instead of two. Furthermore, the spectra obtained for each titration varies depending on the metal being added to solution (Figure 38). Likely the three products observed throughout each titration are due to the availability of multiple coordination modes for $\mathbf{T} 2$.

At low concentrations a given metal salt results in a bright-yellow solution and a red-shifted spectrum which is unique to the metal added. Initially copper(II) forms a complex which has the most blue-shifted absorption spectrum with $\lambda_{\max }$ at $372 \mathrm{~nm}$, while mercury(II), iron(III) and lead(II) form complexes with absorption peaks at $399 \mathrm{~nm}$, $407 \mathrm{~nm}$, and $422 \mathrm{~nm}$ respectively. An isosbestic point is observed during titrations with copper(II), iron(III), and lead(II) suggesting a direct conversion to one metal complex at low concentrations of metal. Higher concentrations of metal result in loss of the isosbestic point, indicative of several products formed in solution. 
Table 9: Absorbance $\lambda_{\max }$ of the three products formed with $\mathbf{T} 2$ and either copper(II), iron(III), lead(II) or mercury(II) in $\mathrm{CH}_{3} \mathrm{CN}$ at $1 \times 10^{-5} \mathrm{M}$.

\begin{tabular}{cccc}
\hline Metal & $\mathbf{1}^{\text {st }}$ Product $(\mathbf{n m})$ & $\mathbf{2}^{\text {nd }}$ Product $(\mathbf{n m})$ & $\mathbf{3}^{\text {rd }}$ Product (nm) \\
\hline Copper(II) & 372 & 391 & 347 \\
Iron(III) & 497 & 394 & 347 \\
Lead(II) & 422 & 399 & 408 \\
Mercury(II) & 399 & 395 & 417 \\
\hline
\end{tabular}

Though at low concentrations a distinct $\lambda_{\max }$ is observed with each metal, increasing concentrations of a metal salt result in similar spectra with nearly identical $\lambda_{\max }$ for the second product. The formation of the second product results in a decrease in absorption intensity and concurrent blue-shift in the $\lambda_{\max }$ for iron(III), lead(II) and mercury(II) (Figure 38). Copper(II) is the only metal which results in a red-shifted $\lambda_{\max }$ resulting in similar spectra for all four metal salts in these mid-concentration ranges. The final product for copper(II) and iron(III) is observed to have a hypsochromic shift in $\lambda_{\max }$, while a bathochromic shift in $\lambda_{\max }$ is observed for lead(II) and mercury(II) (Figure 38). $\mathrm{T} 2$ is a rare example of a ligand capable of forming three different complexes in solution with increasing concentration of metal (Table 9). The ability to form three distinct 103 
products suggests this sensor is capable of detecting a large range of concentrations for any of these metals, ideal for sensing applications.
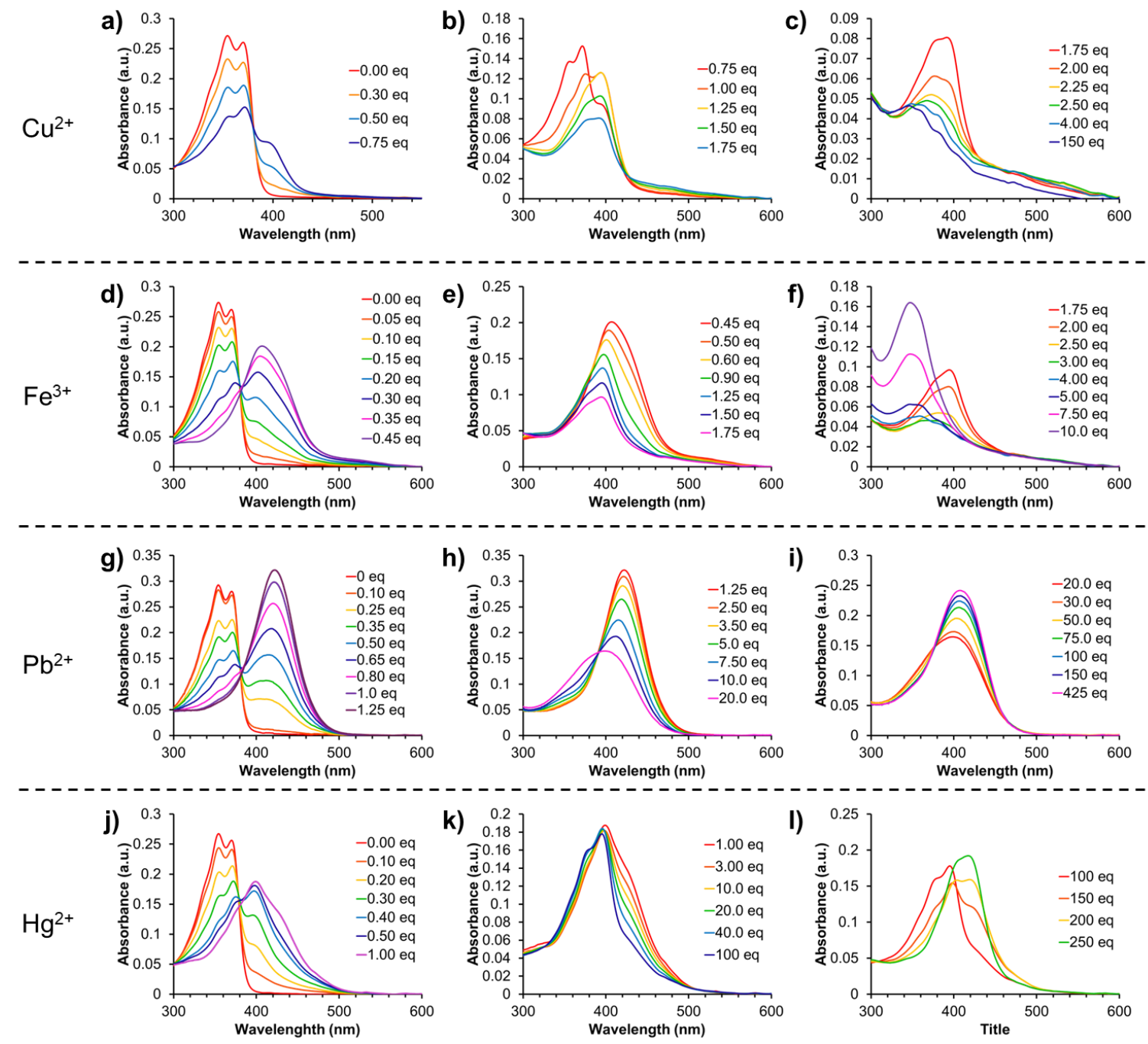

Figure 38: Absorption titrations of T2 with copper(II) (a-c), iron(III) (d-f), lead(II) (g-i), and mercury(II) $(\mathrm{j}-1)$. 

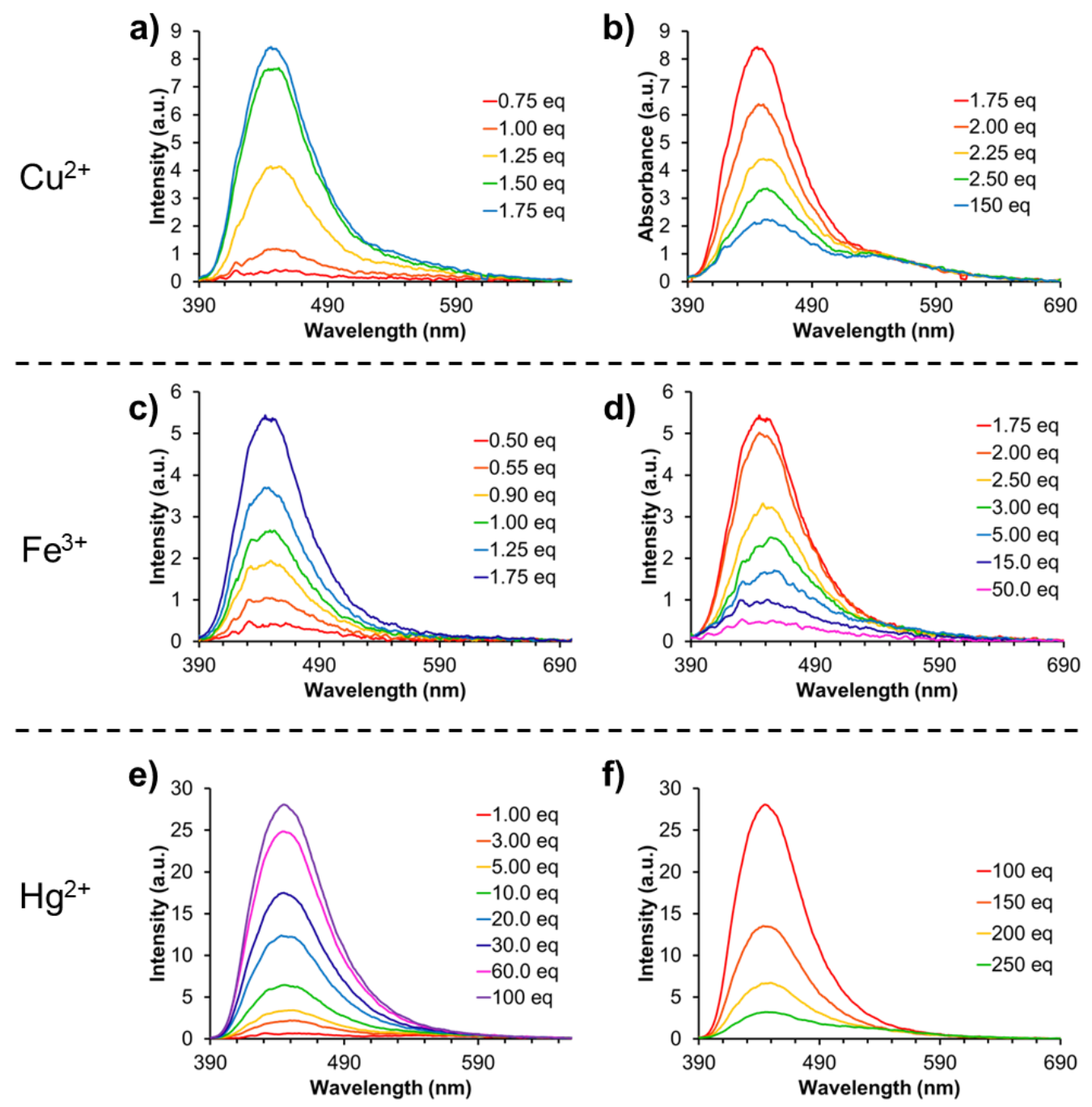

Figure 39: Change in emission of $\mathbf{T} 2$ with increasing amounts of copper(II) (a-b), iron(III) (c-d), and mercury(II) (e-f) in $\mathrm{CH}_{3} \mathrm{CN}$ at $1 \times 10^{-5} \mathrm{M}$.

The ligand $\mathbf{T 2}$ is not emissive in solution nor is the first complex formed, as determined by simultaneous absorption and emission titrations. However formation of the second product results in an emissive complex with increasing amounts of copper(II), iron(III), and mercury(II), effectively acting as a 'turn-on' sensor. After the addition of $0.75,0.50$, and 1.00 equivalent of copper(II), iron(III) and mercury(II), respectively, this 
emissive product is observed (Figure 39). Following formation of the emissive product, all complex emissions are quenched with greater additions of the metal salt. Lead(II) is the only metal which did not form an emissive product. Though an emissive product is observed for copper(II), iron(III), and mercury(II) there is no difference between the complex emission for each metal salt $\left(\lambda_{\max }=450 \mathrm{~nm}\right)$. The formation of an identical emission for these metals suggests a similar coordination is adopted regardless of the metal ion suppressing the quenching pathway of $\mathbf{T} 2$.

The unique photophysical responses of $\mathbf{T} 2$ at low concentrations of copper(II), iron(III), lead(II) and mercury(II) suggest that various coordination modes are occurring between each metal. To confirm this ${ }^{1} \mathrm{H}$ NMR experiments were performed. In the case of a monometallic complex one of three possible coordination modes can occur with these sensors as previously discussed (Figure 31). In the case of chelation via the pyridyl nitrogen and heteroatom of the functional group to a single metal (Figure 31a) there will be a break in symmetry of the molecule that will cause an increase in the number of peaks observed. When the heteroatoms of the functional groups themselves chelate (Figure 31b) the largest downfield shift will be observed for the functional group peaks. Of the possible coordination modes the most challenging to identify will be $\mathrm{N}, \mathrm{S}, \mathrm{N}$-chelation (Figure 31c), since all sites of the thiophene ring have been substituted. However this coordination mode can be inferred by an observed shift in the pyridyl peaks with retention in the number of peaks, as observed in $\mathbf{T 1}$. 

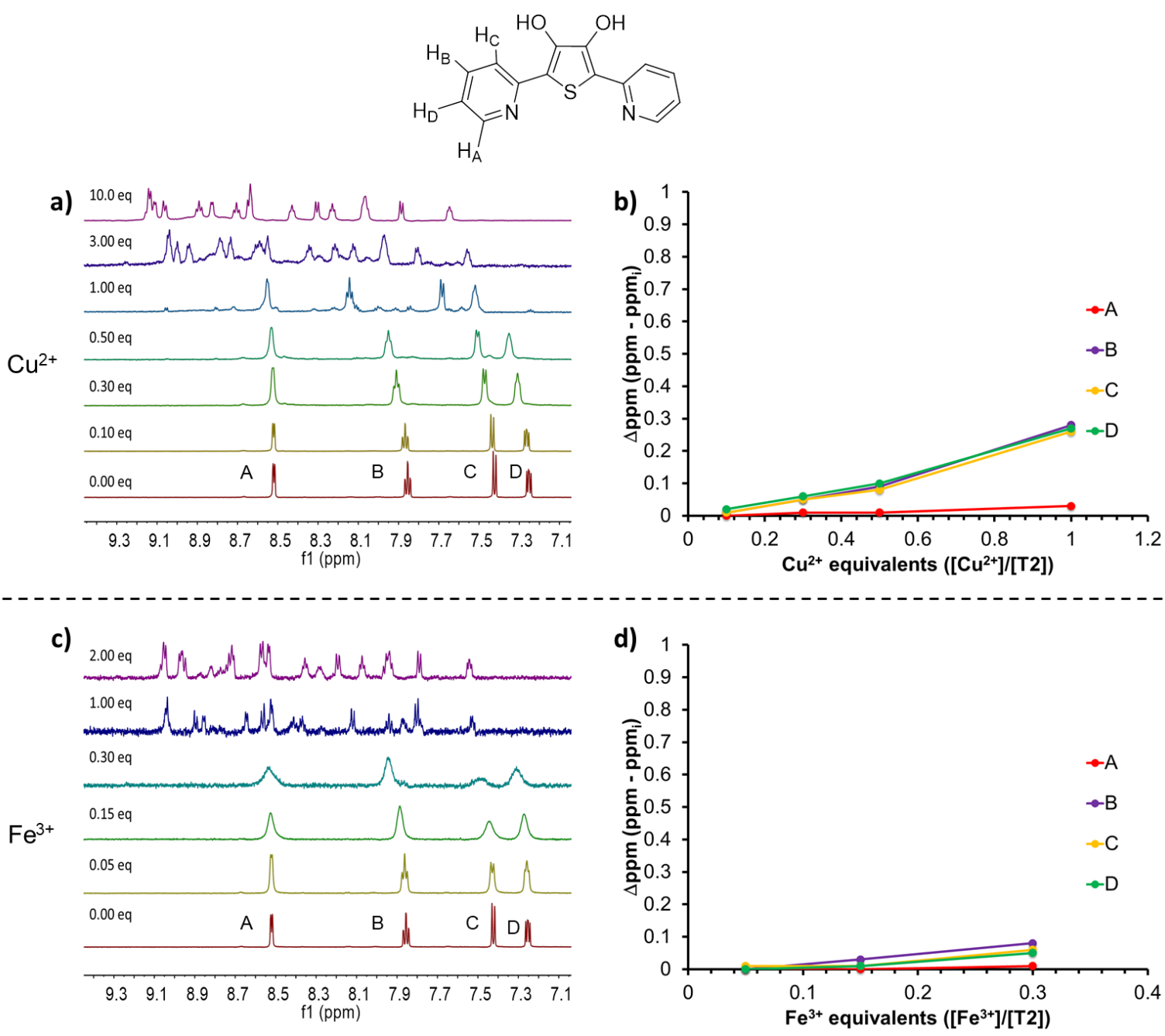

Figure 40: ${ }^{1} \mathrm{H}$ NMR titration of $\mathbf{T} 2$ at $4 \times 10^{-3} \mathrm{M}$ with increasing amounts of copper(II) and iron(III) in $\mathrm{CD}_{3} \mathrm{CN}$. The magnitude of the chemical shift is plotted for each peak with increasing equivalents of (b) copper(II), and (d) iron(III).

Low concentrations of copper(II) and iron(III) result in a minor downfield shift and broadening of the pyridyl peaks (Figure $40 \mathrm{~b} \& \mathrm{~d}$ ), with simultaneous disappearance of the alcohol peak. This suggests that initial coordination to a copper(II) and iron(III) metal center occurs primarily through the alcohol oxygens. Though $\mathbf{T} 2$ adopts identical coordination modes with copper(II) and iron(III) a specific absorption response is observed at low concentrations of these metals. The observed differences in the 107 
absorption spectrum could be attributed to a difference in molecular geometry about the metal center.

Greater than 0.30 equivalents of copper(II) and iron(III) result in a downfield shift of the pyridyl peaks, and an increase in the number of aromatic peaks. A total of 12 peaks are observed at high concentrations of copper(II) and iron(III) respectively (Figure 40). The integrations of these peaks are approximately equivalent. An increase in the number of peaks suggests a break in symmetry similar to N,O-chelation, however there should only be a total of 8 peaks in this case. Thus multiple species must be present in solution, as suggested in the absorption titrations at higher concentrations. In the case of iron(III) the number of peaks could be explained by up to two species in solution, where one species present is due to N,O-chelation of $\mathbf{T} 2$ accounting for 8 peaks. The remaining 4 peaks are likely due to N,S,N-chelation of the thienyl sulfur and pyridyl nitrogen, consistent with the downfield shifted peaks observed. In the case of copper(II) I expected peak broadening from the paramagnetic metal center however this was not observed. Given the high relative concentration of copper(II) when these peaks are observed it seems plausible that a side reaction could be occurring between copper(II) and T2, resulting in the observed peaks.

Unlike copper(II) or iron(III), low concentrations of mercury(II) result in a downfield shift of the pyridyl peaks, suggesting initial coordination of the pyridyl nitrogen (Figure 41). Given the number of peaks is retained throughout the titration I believe tridentate coordination of the thienyl sulfur and pyridyl nitrogen is occurring. This is consistent with the observed absorption spectrum with mercury(II) which is 108 
distinct from the one observed after addition of copper(II) or iron(III). Rather severe broadening of the aromatic pyridyl peaks at 5.00 equivalents suggests exchange is occurring making it difficult to determine the number of pyridyl peaks. Though four distinct peaks appear present a number of minor impurities can also be observed in the aromatic region, consistent with multiple species in solution. Greater concentrations of mercury(II) result in sharpening of the pyridyl peaks and generation of several peaks at low intensity. There appear to be several additional peaks present at a low intensity, consistent with chelation of the alcohol oxygens. The large amounts of mercury(II) required to form these products are likely due to the fact that the alcohol oxygen is a hard Lewis base, and therefore not capable of forming a stable coordinate bond with the soft mercury(II) ion. These large concentrations needed to generate this product are also consistent with the high concentrations needed to form later products during absorption and emission titrations. 

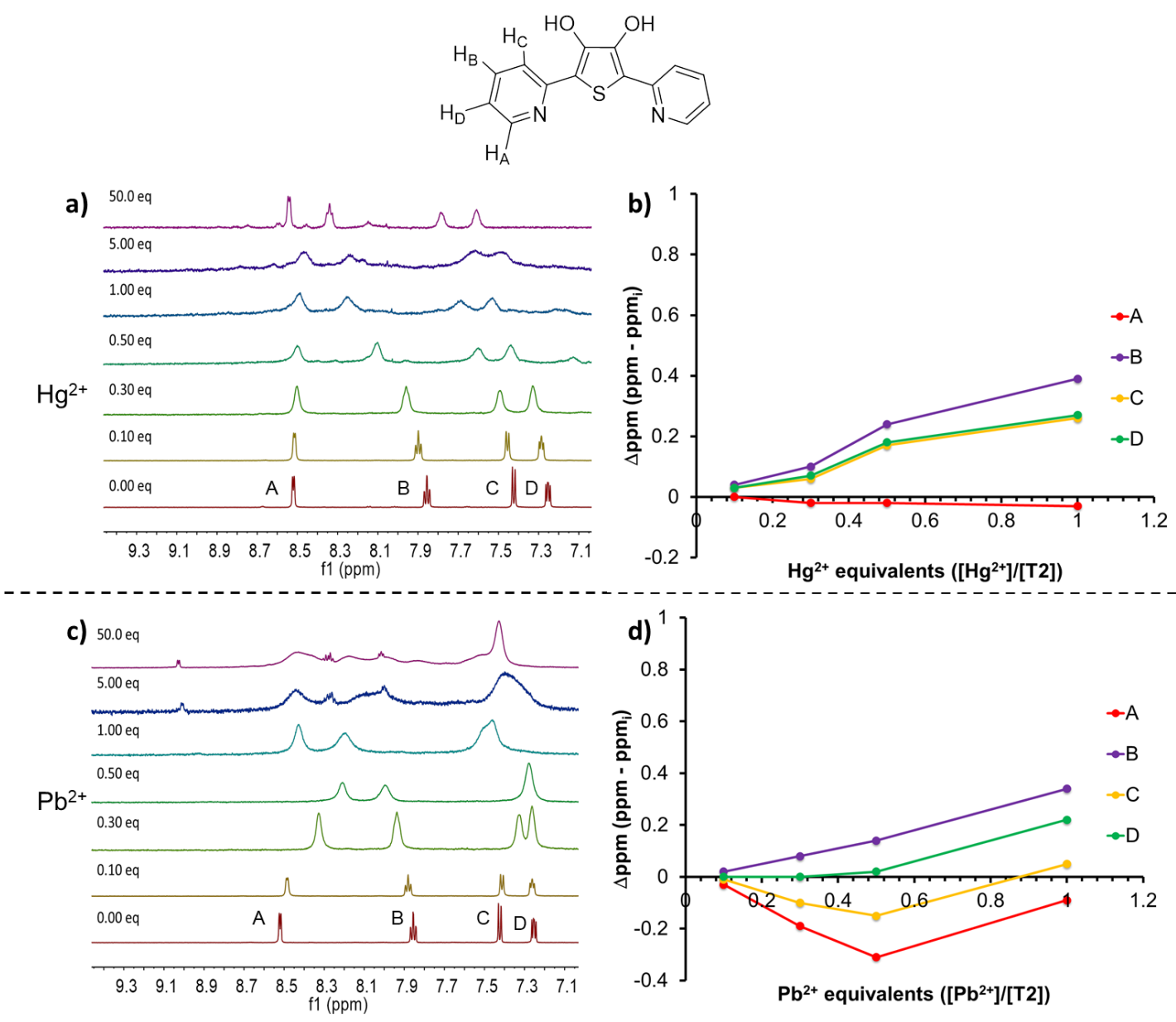

Figure 41: ${ }^{1} \mathrm{H}$ NMR titration of T2 at $4 \times 10^{-3} \mathrm{M}$ with increasing amounts of lead(II) and mercury(III) in $\mathrm{CD}_{3} \mathrm{CN}$. The magnitude of the chemical shift is plotted for each peak with increasing equivalents of (b) lead(II), and (d) mercury(II).

Lead(II) results in the most unique changes in the ${ }^{1} \mathrm{H}$ NMR spectrum of $\mathbf{T} 2$. Low concentrations of lead(II) result in an upfield shift of two of the pyridyl peaks, while a downfield shift is observed for the remaining peaks suggesting N,S,N-chelation. An upfield shift of the pyridyl peaks suggests that there is an increase in electron density about these proton environments. This can be explained by $\pi$-backbonding of the metal to the ligand, which causes an increase in electron density about the pyridyl ring. Though mercury(II) and lead(II) appear to adopt similar coordination modes they result in 
different absorption spectra, at low concentrations, upon binding of T2. Likely this is due to the observed $\pi$-backbonding that occurs with lead(II). With greater amounts of lead(II) all pyridyl peaks begin to shift downfield, and broaden severely. Similar to mercury(II) broadening of the pyridyl peaks occurs with simultaneous increase of several other aromatic peaks at low intensity suggesting multiple species form in solution. The extreme broadening of the pyridyl peaks make it difficult to determine which coordination modes are occurring, though the presence of multiple species is consistent with absorption titrations where several products were observed to form. As I have shown from these ${ }^{1} \mathrm{H}$ NMR experiments, the availability of the multiple coordination modes have facilitated the formation of multiple products in solution.
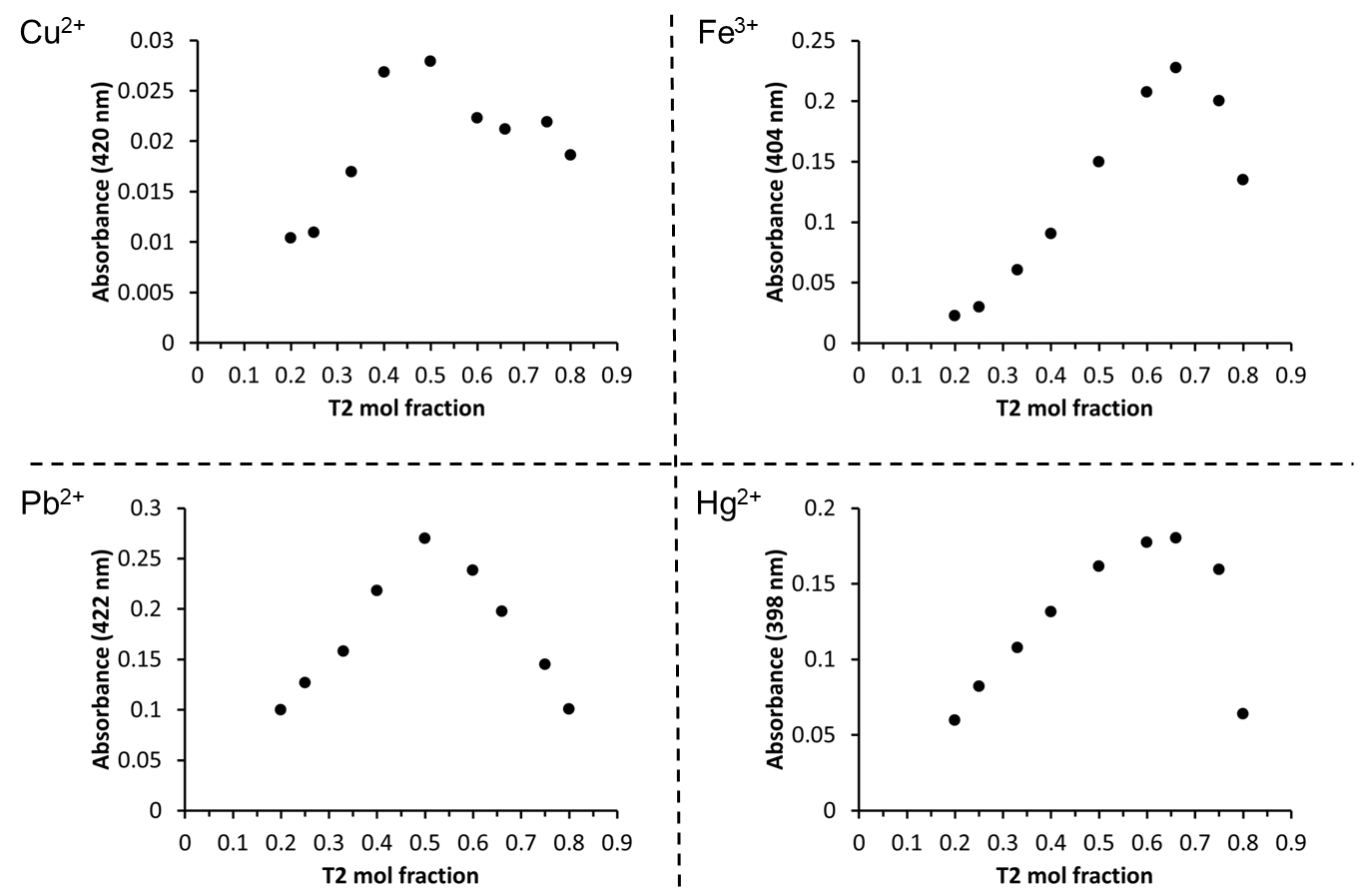

Figure 42: Absorbance Job's plots obtained with T2 and copper(II), iron(III), lead(II) and mercury(II) in $\mathrm{CH}_{3} \mathrm{CN}$ at a total concentration of $2 \times 10^{-5} \mathrm{M}$. 
According to Job's plots obtained for the four metals tested, both copper(II) and lead(II) bind in a 1:1 stoichiometry, while mercury(II) and iron(III) form complexes with a 2:1 stoichiometry of sensor to metal (Figure 42). Association constants could only be obtained from the absorption titrations of copper(II), iron(III) and mercury(II) (Table 10). T2 has the greatest association constant for copper(II), iron(III), and mercury(II) out of the sensors tested. The best fits obtained from the absorption titration data with these three metals suggest all bind in a 2:1 stoichiometry. An appropriate fit could not be obtained for the lead(II) titration data with various models, suggesting complicating factors in interpreting this data. However, the Job's plot for lead(II) suggested a 1:1 stoichiometry. As expected, functionalization of the thienyl moiety with alcohol groups causes an increase in the $K_{\mathrm{a}}$ of $\mathbf{L 2}$ from $5.37 \times 10^{9} \mathrm{M}^{-2}( \pm 9.8 \%)$ to $9.76 \times 10^{11} \mathrm{M}^{-2}( \pm 25.6 \%)$. The observed $K_{\mathrm{a}}$ of $\mathbf{T} 2\left(9.76 \times 10^{11} \mathrm{M}^{-2} \pm 25.6 \%\right)$ is greater than that of $\mathbf{P 1}\left(4.52 \times 10^{10} \pm 18 \%\right)$, described in the previous chapter, where the alcohol groups were added to the pyridyl rings of $\mathbf{L} \mathbf{2}$ instead.

Table 10: Association constant data for the binding of $\mathbf{T} 2$ to copper(II), iron(III), and mercury(II) in $\mathrm{CH}_{3} \mathrm{CN}$ obtained from absorption titrations.

\begin{tabular}{cc}
\hline Metal ion & $\boldsymbol{K}_{\mathbf{a}}$ \\
\hline Copper(II) & $2.14 \times 10^{12} \mathrm{M}^{-2}( \pm 26.4 \%)$ \\
Iron(III) & $8.28 \times 10^{11} \mathrm{M}^{-2}( \pm 15.3 \%)$ \\
Lead(II) & $\mathrm{N} / \mathrm{A}$ \\
\hline
\end{tabular}


Competition experiments were performed to determine the selectivity of $\mathbf{T} 2$ for copper(II), iron(III), lead(II) and mercury(II) against other transition metal ions. Not surprisingly, based on the strong response to the tested metals, $\mathbf{T} 2$ also has an absorption response for several other metal ions including: cadmium(II), cobalt(II), iron(II), magnesium(II), manganese(II) and zinc(II). Subsequent addition of lead(II) and mercury(II) to these solutions results in an absorption spectrum comparable to the one obtained with only lead(II) or mercury(II). In the case of lead(II) a generally selective response is obtained however competitive binding appears to occur with both copper(II) and iron(III). Similarly competitive binding is observed with mercury(II), as the presence of copper(II), iron(III) and zinc(II) prevents generation of the absorption spectrum observed with just mercury(II). Thus functionalization of the thiophene ring with the alcohol group has not appeared to generate the desired selectivity for soft Lewis acids, as the increased binding affinity has resulted in a decreased selectivity similar to the pyridine-functionalized sensors. However the position of the functional group does facilitate the formation of several products in solution allowing for a larger range of detectable concentrations. Furthermore the specific responses obtained for the four metals tested suggests that $\mathbf{T} 2$ could be a useful model for the development of a multiplex sensor. 

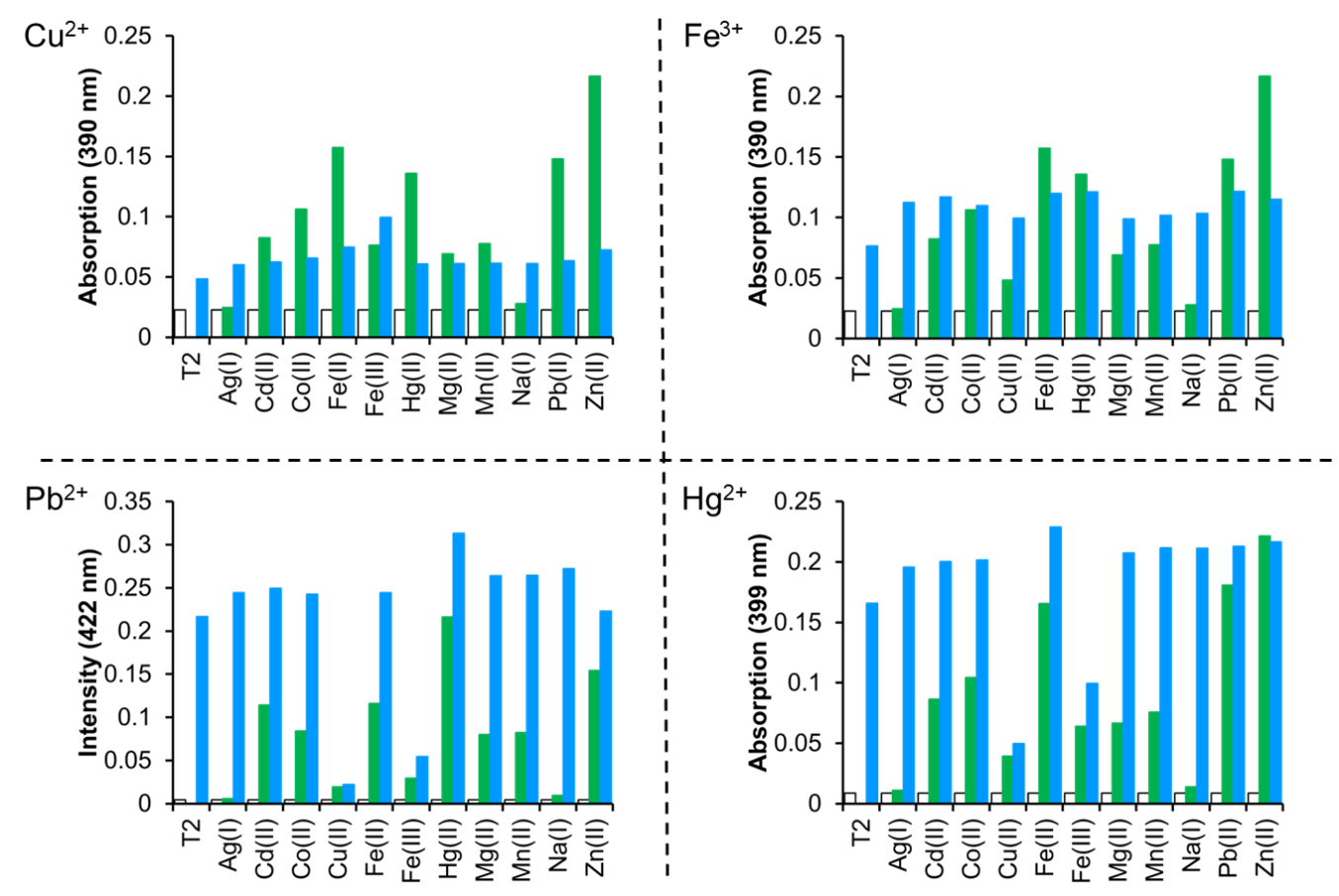

Figure 43: Absorption at 390, 422, and $399 \mathrm{~nm}$ of T2 (white bars), after the addition of 5 eq. of metal ion (green bars), and subsequent addition of 5 eq. of either copper(II), iron(III), lead(II) or mercury(II) perchlorate (blue bars) in $\mathrm{CH}_{3} \mathrm{CN}$.

\subsubsection{Reactivity of $\mathbf{T 3}$ to $\mathrm{Cu}^{2+}, \mathrm{Fe}^{3+}, \mathrm{Pb}^{2+}$ and $\mathrm{Hg}^{2+}$}

Though $\mathbf{T 1}$ and $\mathbf{T 3}$ are similar in terms of structure a few distinctions are noted in their photophysical response with the addition of each metal salt. A unique absorption and emission response is only obtained in the case of mercury(II) and lead(II), as compared to the responses observed for copper(II) and iron(III). Furthermore the presence of sodium(I) appears to alter the photophysical response of $\mathbf{T 3}$ to copper(II). The observed $K_{\mathrm{a}}$ values obtained for mercury(II) and lead(II) have also notably increased relative to $\mathbf{L 2}$, unlike $\mathbf{T} 1$. 
Similar to $\mathbf{T 1}$ the addition of metal salts results in the formation of two distinct products for the titrations of $\mathbf{T 2}$ with copper(II), iron(III), mercury(II) and lead(II) (Figure 44). Copper(II) and iron(III) result in similar absorption spectra at low concentrations upon binding to $\mathbf{T 3}\left(\lambda_{\max }=382 \mathrm{~nm}\right)$, while low concentrations of mercury(II) result in a spectrum with $\lambda_{\max }$ at $369 \mathrm{~nm}$. $\mathbf{T 3}$ responds to as little as $0.19 \mathrm{ppm}$ of copper(II) and is therefore able to meet EPA standards (1.3 ppm). Lead(II) is the only metal which causes a hypsochromic shift in the absorbance spectrum at low concentrations $\left(\lambda_{\max }=341 \mathrm{~nm}\right)$. Increasing amounts of copper(II) and iron(III) result in a blue-shift in the spectrum with similar $\lambda_{\max }$ to each other $\left(\mathrm{Cu}^{2+}: 373 \mathrm{~nm} ; \mathrm{Fe}^{3+}: 368 \mathrm{~nm}\right)$, while greater concentrations of lead(II) cause a ratiometric red-shift to a new $\lambda_{\max }$ at $373 \mathrm{~nm}$. Though no change in $\lambda_{\max }$ is observed with increasing equivalents of mercury(II) the absorption intensity does decrease. 

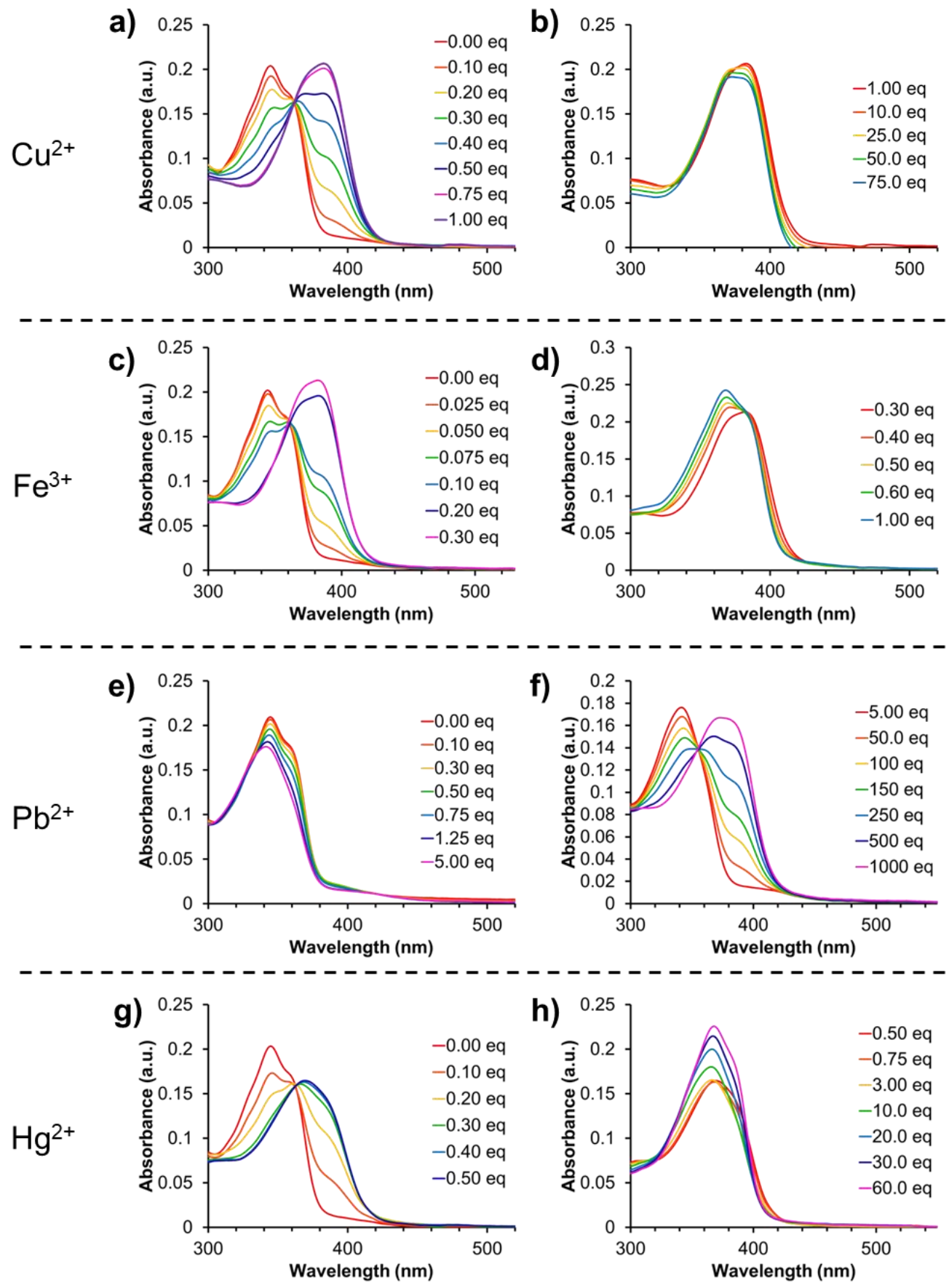

Figure 44: Change in absorption spectrum of $\mathbf{T 3}$ with increasing amounts of copper(II) (a-b), iron(III) (c-d), lead(II) (e-f), and mercury(II) in $\mathrm{CH}_{3} \mathrm{CN}$ at $2 \times 10^{-5} \mathrm{M}$. 
Similar to the absorption response, there is little distinction between the emission spectrum of the complexes formed with copper(II), iron(III) and mercury(II) (Figure 45). The growth of a red-shifted peak in the emission spectrum $\left(\lambda_{\max }=468 \mathrm{~nm}\right)$ is observed at low concentrations for all metals except lead(II). Greater amounts of copper(II), iron(III), and mercury(II) result in a blue-shift in the emission spectrum. At low concentrations, lead(II) causes quenching of the emission with no change in $\lambda_{\max }$ however once more then 5.00 equivalents have been added a ratiometric response is observed similar to $\mathbf{T 1}$ (Figure 45f). As mentioned previously this is a rare response for this metal, and gives good precedence for using $\mathbf{T 1}$ and $\mathbf{T 3}$ as a model for the development of a ratiometric fluorescent lead(II) sensor. 

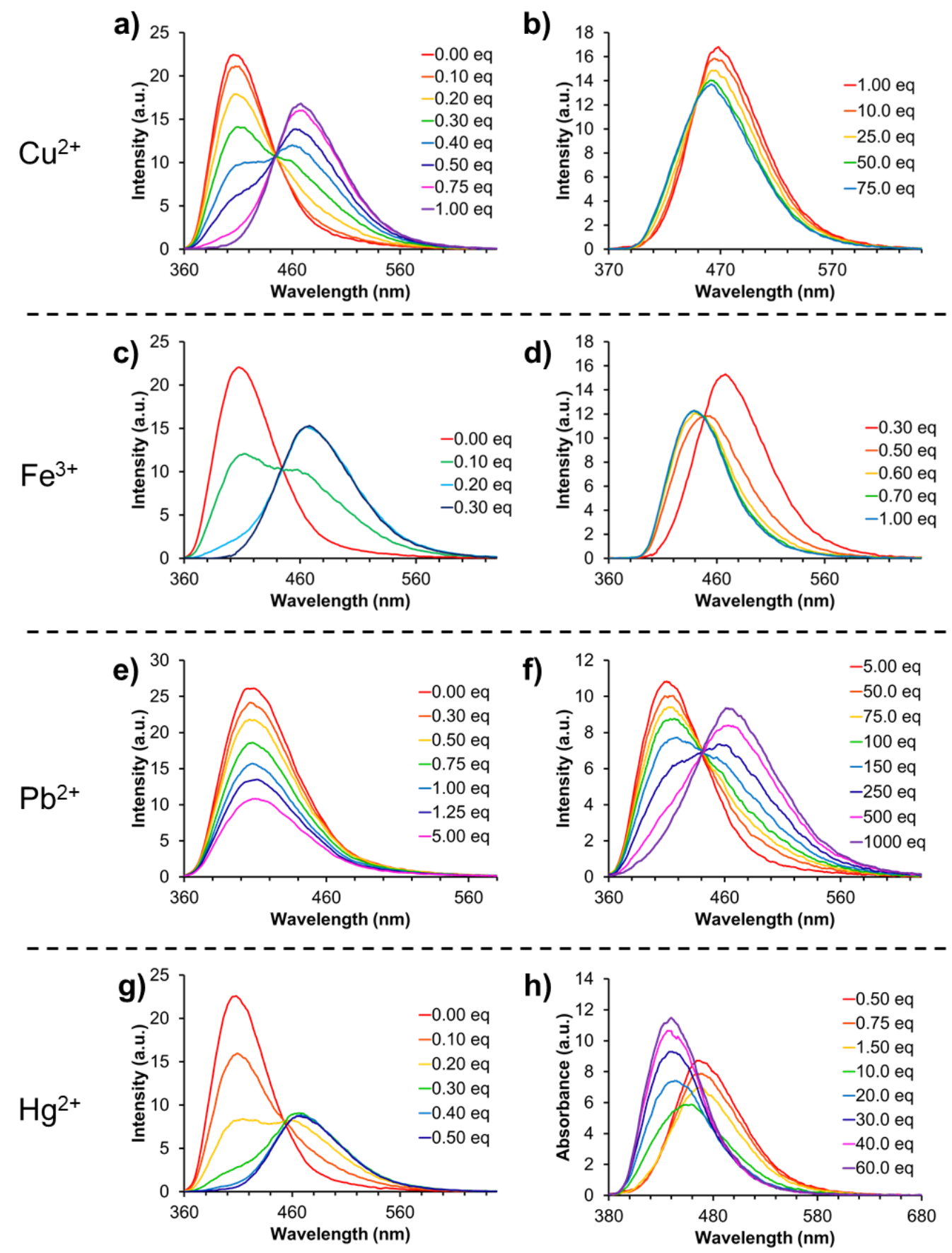

Figure 45: Change in emission spectrum of T3 with increasing amounts of copper(II) (ab), iron(III) (c-d), lead(II) (e-f) and mercury(II) (g-h) in $\mathrm{CH}_{3} \mathrm{CN}$ at $2 \times 10-5 \mathrm{M}$. All solutions were excited at $350 \mathrm{~nm}$. 

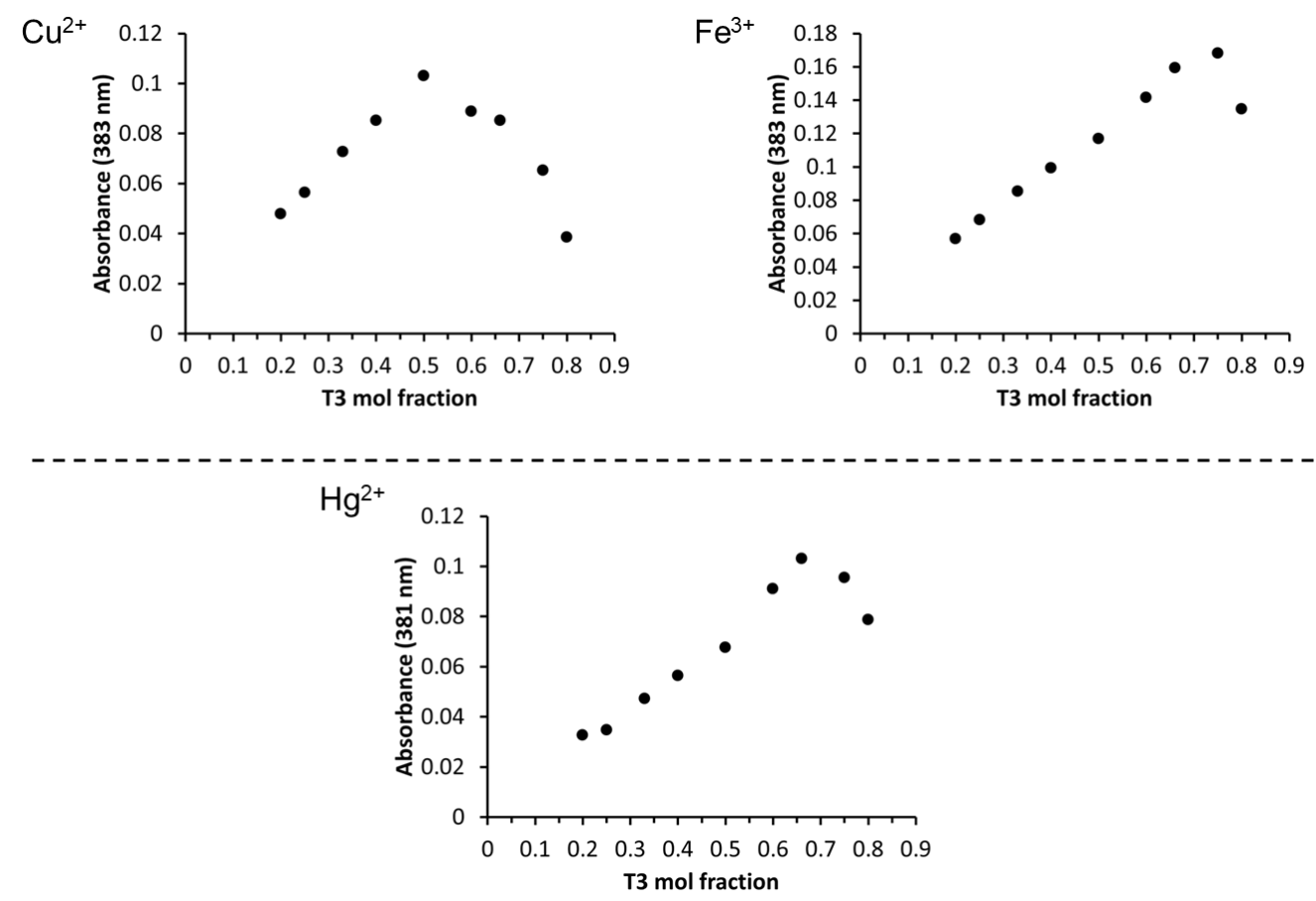

Figure 46: Job's plot obtained for T3 with copper(II), iron(III), and mercury(II) in $\mathrm{CH}_{3} \mathrm{CN}$ at a total concentration of $2 \times 10^{-5} \mathrm{M}$.

Job's plots were obtained for all four metals, except for lead(II) due to the inability to differentiate the $\lambda_{\max }$ of the lead(II)-complex from that of T3. According to the fitting analysis of the absorption titration data, both mercury(II) and lead(II) bind at a 2:1 stoichiometry (T3:metal) initially at low concentrations of metal. Though the Job's plot for copper(II) suggests a 1:1 stoichiometry no suitable fit to the titration data could be obtained with the models tested. Similarly, no association constant could be obtained for the complex formed with iron(III) and T3, however the Job's plot suggests a unique 3:1 stoichiometry. Though T1 and T3 bear similar functional groups, an increase in the association constant for mercury(II) is observed for $\mathbf{T 3}\left(6.66 \times 10^{12} \mathbf{M}^{-2} \pm 13.0 \%\right)$ relative to $\mathbf{L} 2\left(5.37 \times 10^{9} \mathrm{M}^{-2} \pm 9.8 \%\right)$ unlike with $\mathbf{T 1}\left(4.34 \times 10^{9} \mathrm{M}^{-2} \pm 13.8 \%\right)$. 
Table 11: Association constants for T3 with copper(II), iron(III), lead(II), and mercury(II) in $\mathrm{CH}_{3} \mathrm{CN}$ obtained from absorption titrations.

\begin{tabular}{cc}
\hline Metal ion & $\boldsymbol{K}_{\mathbf{a}}$ \\
\hline Copper(II) & N/A \\
Iron(III) & N/A \\
Lead(II) & $7.97 \times 10^{7} \mathrm{M}^{-2}( \pm 8.32 \%)$ \\
Mercury(II) & $6.66 \times 10^{12} \mathrm{M}^{-2}( \pm 13.0 \%)$ \\
\hline
\end{tabular}

In addition to the photophysical responses described thus far a unique response to copper(II) was observed in the presence of $1 \times 10^{-4} \mathrm{M}$ of sodium(I). When copper(II) is added to a solution of $\mathbf{T 3}$ in the presence of sodium(I) a pink solution that has two redshifted transitions in the absorbance spectrum at $366 \mathrm{~nm}$ and $531 \mathrm{~nm}$ is observed (Figure 47). Of the metal ions tested copper(II) was the only metal to elicit this response in the presence of sodium(I). I believe this unique response to be due to the formation of a heterobimetallic complex with both sodium(I) and copper(II). Likely sodium(I) coordinates to the octaethyleneglycol monomethyl ether chains while copper(II) coordinates via N,S,N-chelation of the pyridyl and thienyl rings. 


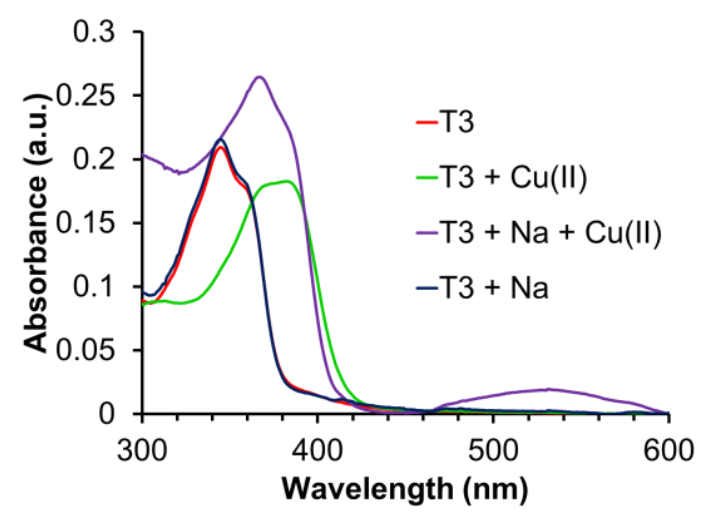

Figure 47: Absorbance spectrum of $\mathbf{T 3}$ at $2 \times 10^{-5} \mathrm{M}$ (red), $\mathbf{T 3}$ and 5 eq. of copper(II) (green), $\mathbf{T 3}$ and 5 eq. of sodium(I) (blue), and $\mathbf{T 3}$ with 5 eq. of both sodium(I) and copper(II) in $\mathrm{CH}_{3} \mathrm{CN}$.

To investigate formation of a heterobimetallic complex between sodium(I) and copper(II), ${ }^{1} \mathrm{H}$ NMR experiments were performed. Addition of sodium(I) to T3 results in a simultaneous downfield shift of the octaethyleneglycol monomethyl ether peaks and an upfield shift of the pyridyl peaks (Figure 48). The observed downfield shift of the functional group peaks is consistent with chelation of two or several oxygens of the octaethylene glycol monomethyl ether groups. It is unclear why an upfield shift of the pyridyl peaks is observed. Subsequent addition of copper(II) results in broadening and a downfield shift of the pyridyl peaks suggesting $\mathrm{N}, \mathrm{S}, \mathrm{N}$-chelation is occurring. The observed broadening is consistent with coordination to a paramagnetic metal. Broadening of the functional group peaks is observed as well, though not as severely as the pyridyl peaks suggesting exchange interactions are occurring with the copper(II) ion. Thus it appears that a heterobimetallic complex is formed and responsible for the unique absorption response observed for copper(II) in the presence of sodium(I). Though similar species should form with addition of other metals, a response similar to the one obtained 
with copper(II) is not observed. The reason for this is unclear. In addition to these experiments, ${ }^{1} \mathrm{H}$ NMR was used to determine the coordination modes of $\mathbf{T} 3$ with only copper(II), iron(III), lead(II) or mercury(II).

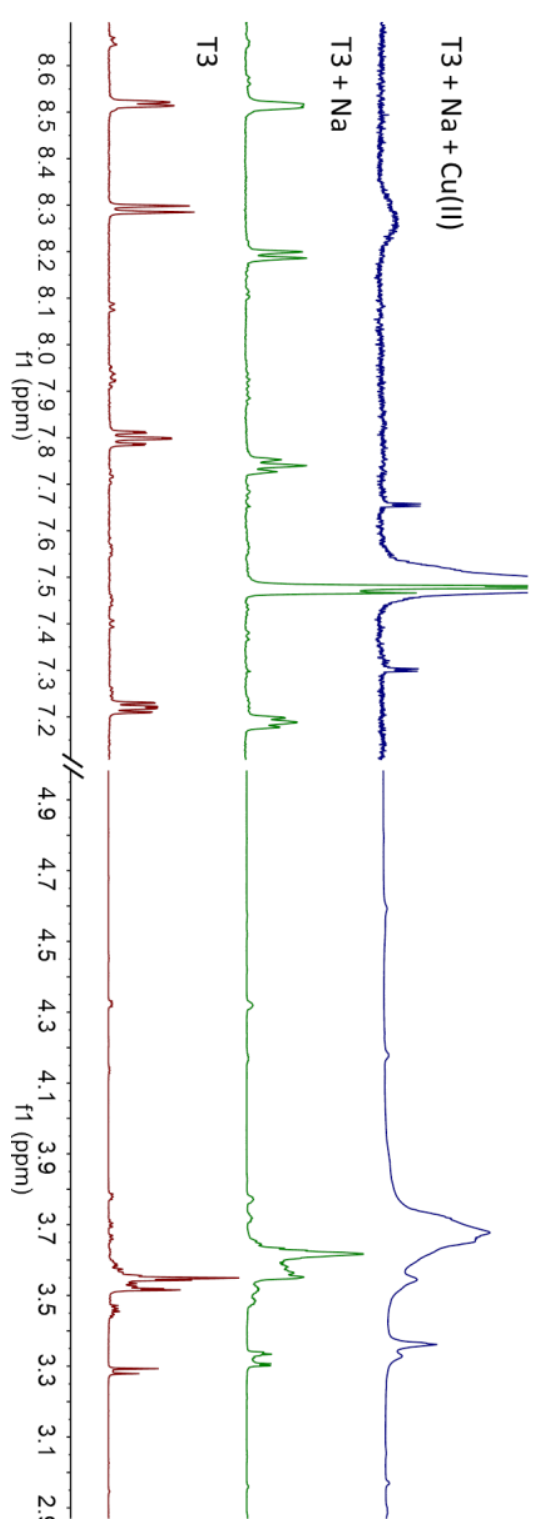

Figure 48: ${ }^{1} \mathrm{H}$ NMR of T3 (red), T3 and 2 equivalents of sodium (green), and T3 in the presence of 2 eq of sodium and 1 eq of copper(II) (blue) in $\mathrm{CD}_{3} \mathrm{CN}$ at $4 \times 10^{-3} \mathrm{M}$. 
The similar photophysical responses of $\mathbf{T} \mathbf{3}$ to the metals tested in this study suggest complexes of similar structure are formed. This is reflected in the ${ }^{1} \mathrm{H}$ NMR experiments performed as increasing amounts of any metal salt results in a gradual downfield shift of the pyridyl peaks, suggesting N,S,N-chelation similar to $\mathbf{L} 2$. Similar to previous ${ }^{1} \mathrm{H}$ NMR experiments, broadening of the pyridyl peaks is observed which suggests exchange interactions are occurring. Notably greater concentrations of the metal salt results in a simultaneous downfield shift of the octaethylene glycol monomethyl ether and pyridyl peaks that suggests chelation of the functional group peaks to a metal center. The two products formed with $\mathbf{T} 1$ and $\mathbf{L} 2$ appear to be the result of N,S,N-chelation at both a 2:1 and 1:1 stoichiometry of sensor to metal. However in the case of $\mathbf{T 3}$ the octaethyleneglycol monomethyl ether peaks appear to form products with a 2:1 and 1:2 stoichiometry (T3:metal) in the case of mercury(II) and lead(II). 


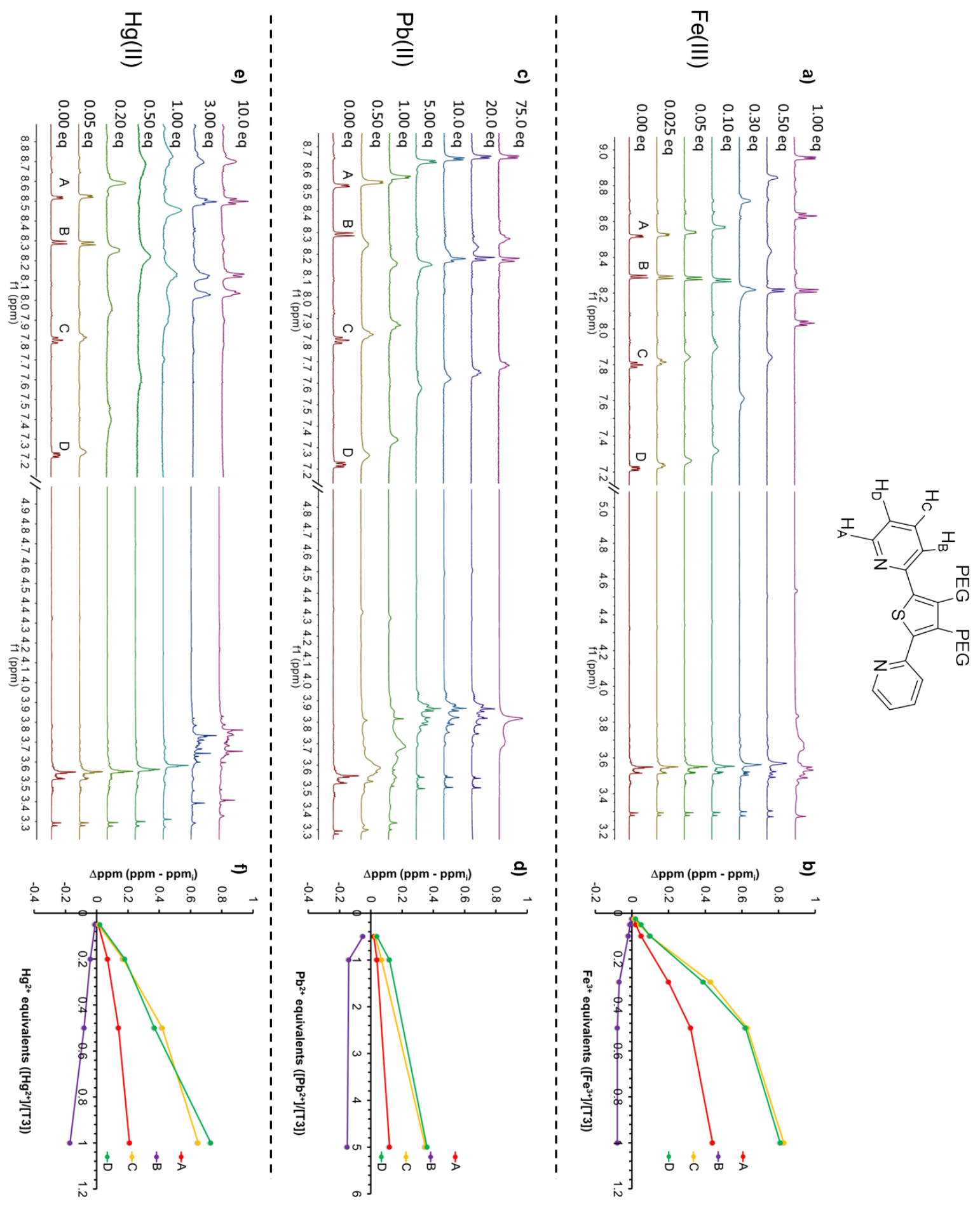

Figure 49: ${ }^{1} \mathrm{H}$ NMR titration of T3 with (a) iron(III), (b) lead(II), and (c) mercury(II) in $\mathrm{CD}_{3} \mathrm{CN}$ at $4 \times 10^{-3} \mathrm{M}$. The magnitude of the chemical shift is plotted for each peak with increasing equivalents of (b) iron(III), (d) lead(II), and (f) mercury(II). 

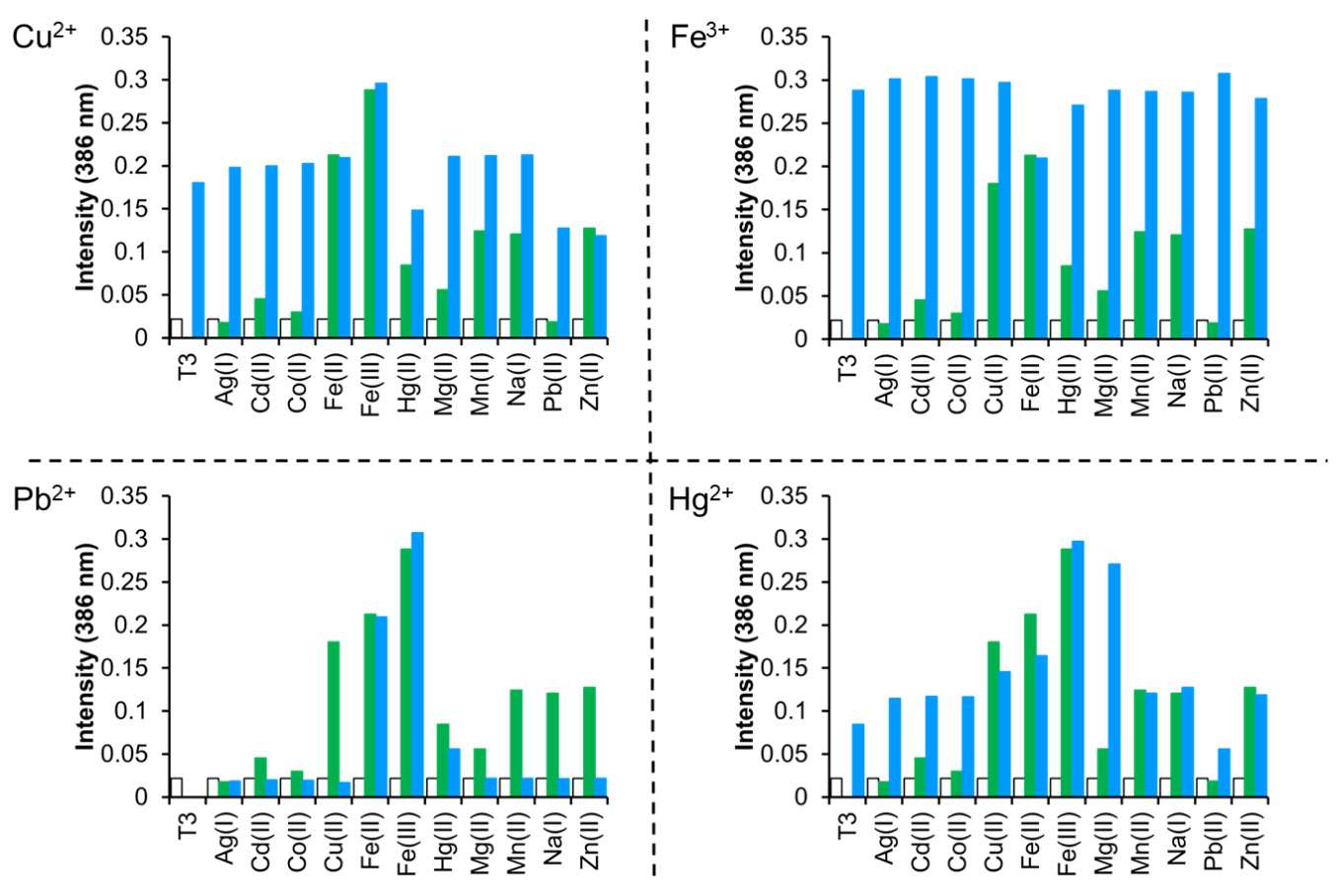

Figure 50: Absorption at $386 \mathrm{~nm}$ of $\mathbf{T 3}$ (white bars), after the addition of 5 eq. of metal ion (green bars), and subsequent addition of 5 eq. of either copper(II), iron(III), lead(II) or mercury(II) perchlorate (blue bars) in $\mathrm{CH}_{3} \mathrm{CN}$.

The ability of $\mathbf{T 3}$ to detect 0.19 ppm of copper(II) suggests that this sensor could be applicable for the detection of copper(II) in either environmental or biological samples. Furthermore the specific response of $\mathbf{T 3}$ in the presence of sodium(I), suggests that $\mathbf{T 3}$ could be useful as a multiplex detector specifically for the selective detection of iron(III) and copper(II) in biological applications where sodium(I) is generally abundant. Of the metals tested an absorption response was observed with iron(II), manganese(II), sodium(I) and zinc(II). In the case of sodium(I) only an increase in the absorption profile of the sensor is observed (Figure 47). Subsequent addition of copper(II) and iron(III) results in apparent displacement of manganese(II), however iron(II) appears to competitively bind with T3. Addition of the octaethyleneglycol monomethyl ether chains 
also did not improve the selectivity for the soft mercury(II) ion, though an increased $K_{\mathrm{a}}$ was observed.

\subsubsection{Reactivity of T4 to $\mathrm{Cu}^{2+}, \mathrm{Fe}^{3+}, \mathrm{Pb}^{2+}$ and $\mathrm{Hg}^{2+}$}

Several distinctions are observed during the titrations of various metal salts with T4. Like previous sensors, low concentrations of copper(II), iron(III), lead(II) and mercury(II) cause a drop in absorption intensity of the ligand peak however, the redshifted peak that grows in appears as shoulder (Figure 51 \&Figure 52). The resulting spectrum of each metal complex appears specific to the metal, however they are not sufficiently separated to differentiate each metal based on $\lambda_{\max }$. Interestingly the number of products observed throughout the titrations appears to be dependent on the metal ion added to solution as well. Only one product is observed during the titration with lead(II), though this could be due to a relatively low $K_{\mathrm{a}}$, as 2500 equivalents are required to form the first product. In contrast multiple products are observed in solution with copper(II), iron(III) and mercury(II). Greater concentrations of copper(II) and iron(III) result in absorption spectra with two separate wavelengths $\left(\mathrm{Cu}^{2+}: 335 \mathrm{~nm}\right.$ and $528 \mathrm{~nm} ; \mathrm{Fe}^{3+}$ : $348 \mathrm{~nm}$ and $493 \mathrm{~nm})$. The isosbestic points observed at lower concentrations of copper(II), and iron(III) are not maintained at higher concentrations suggesting the formation of multiple products in solution as more metal is added. For iron(III) it is possible the isosbestic point is maintained, though it is difficult to determine whether this is true as the absorption of the free metal salt overlaps with the complex absorption and may cause interference as greater concentrations of iron(III) are added. Compared to other metals, mercury(II) is observed to form the greatest number of products as 
evidenced by a series of changes in the absorption and emission spectrum as increased concentrations are added.
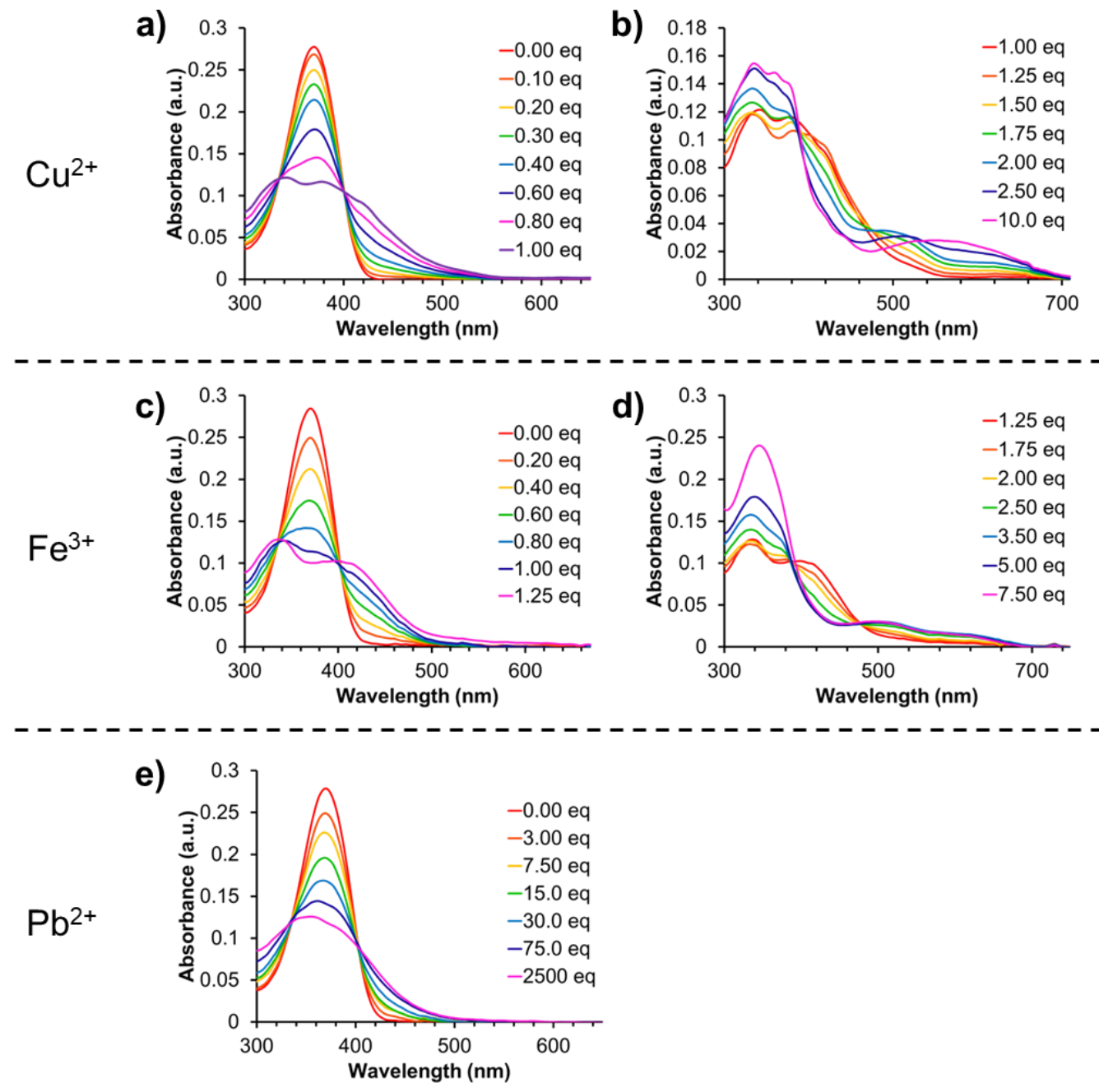

Figure 51: Change in absorbance of $\mathbf{T 4}$ with increasing amounts of copper(II) (a-b), iron(III), (c-d), and lead(II) (e) in $\mathrm{CH}_{3} \mathrm{CN}$ at $1 \times 10^{-5} \mathrm{M}$.

Similar to the other metal salts, low concentrations of mercury(II) result in a decrease in absorption intensity of $\mathbf{T 4}$ with a simultaneous increase of a red-shifted shoulder (Figure 52). As greater than 0.80 equivalents of mercury(II) are added there are 
relatively little changes observed in the absorption spectrum, and a broad featureless spectrum is obtained (Figure 52b). Though only subtle changes are observed in the absorption spectrum in this mid-concentration range, the same concentrations of mercury result in an observable quenching of the emission spectrum (Figure 52e). The lack of a significant change in the absorption spectrum with concurrent change in emission spectrum suggests multiple products are formed in solution with similar absorption spectra resulting in the observed broad spectrum. Greater concentrations of mercury(II) result in a decrease in the shoulder of the spectrum and simultaneous increase in a peak at $350 \mathrm{~nm}$.
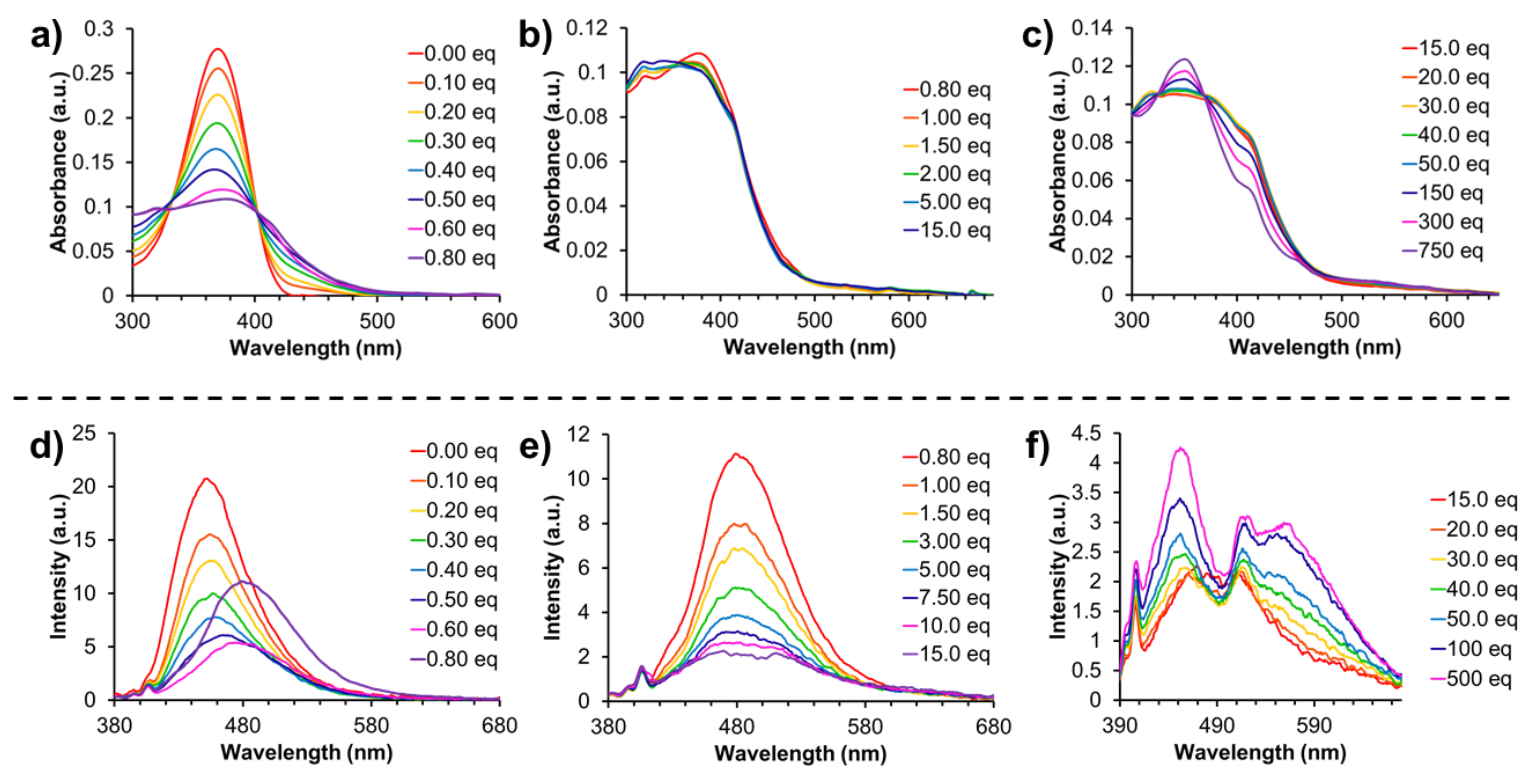

Figure 52: Change in absorption (a-c) and emission (d-f) of T4 in response to increasing amounts of mercury(II) in $\mathrm{CH}_{3} \mathrm{CN}$ at $1 \times 10^{-5} \mathrm{M}$. Emission spectra were obtained by exciting at $370 \mathrm{~nm}$.

For all metal salts, increasing concentrations initially result in quenching of $\mathbf{T 4}$ emission (Figure 53). While lead(II) simply results in complete quenching of emission 
with no change in $\lambda_{\max }$, copper(II) and iron(III) result in a hypsochromic shift of $\lambda_{\max }$ $\left(\mathrm{Cu}^{2+}: 433 \mathrm{~nm} ; \mathrm{Fe}^{3+}: 441 \mathrm{~nm}\right)$. Addition of mercury(II) results in similar quenching however a bathochromic shift of $\lambda_{\max }$ to $479 \mathrm{~nm}$ is observed. Interestingly higher concentrations of copper(II), iron(III) and mercury(II) result in a dual emission response with $\lambda_{\max }\left(\mathrm{Cu}^{2+}: 469 \mathrm{~nm}\right.$ and $590 \mathrm{~nm} ; \mathrm{Fe}^{3+}: 469$ and $577 \mathrm{~nm} ; \mathrm{Hg}^{2+}: 452 \mathrm{~nm}$ and $\left.562 \mathrm{~nm}\right)$. While it is possible this dual emission is the result of a single metal complex in solution, it is more likely that multiple products are formed concurrently in solution resulting in the two discrete peaks observed in the emission spectrum. 

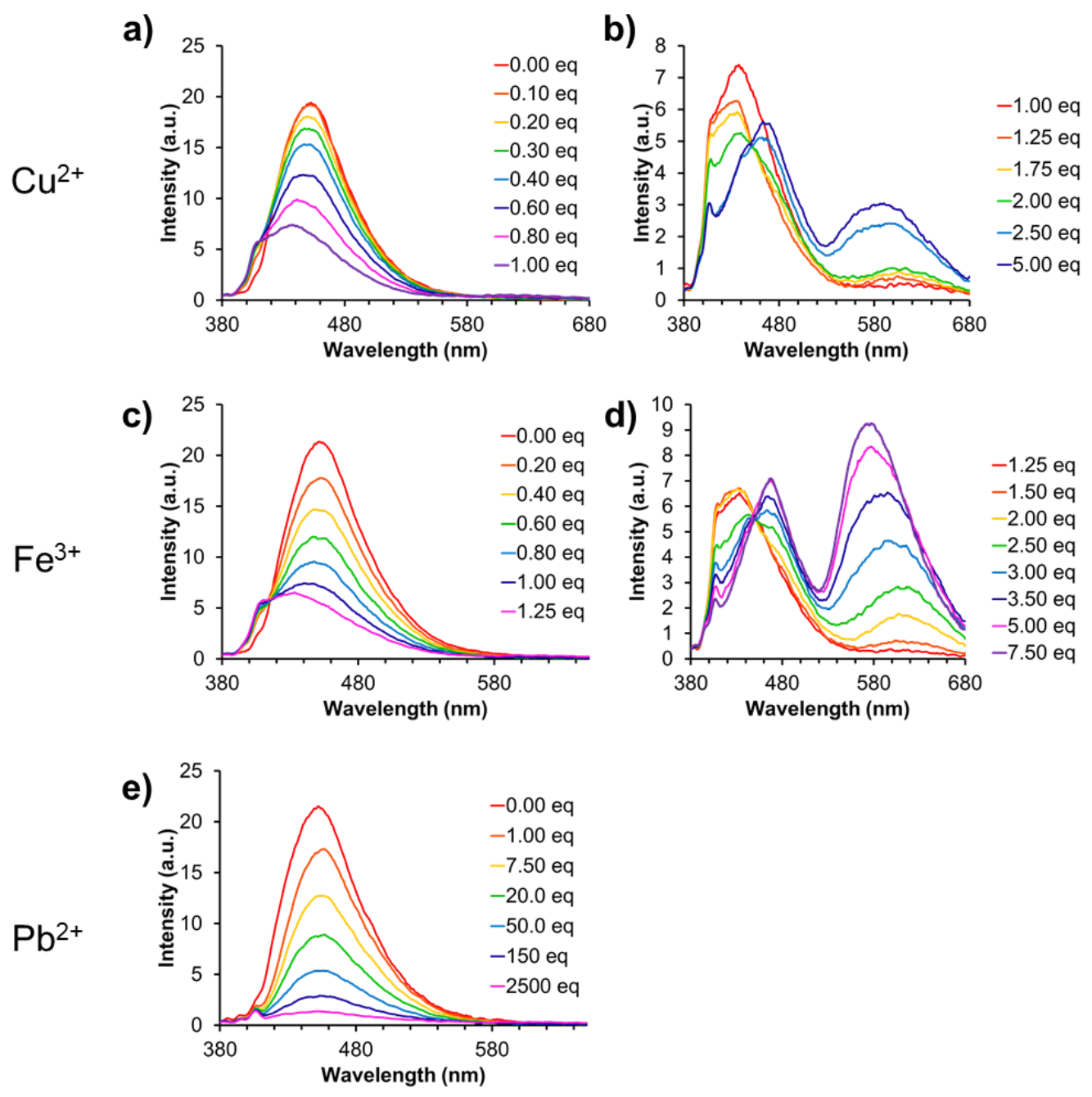

Figure 53: Change in emission spectrum of T4 with increasing amounts of copper(II) (ab), iron(III) (c-d) and lead(II) (e) in $\mathrm{CH}_{3} \mathrm{CN}$ at $1 \times 10^{-5} \mathrm{M}$. All emission spectra were obtained by exciting at $370 \mathrm{~nm}$. 


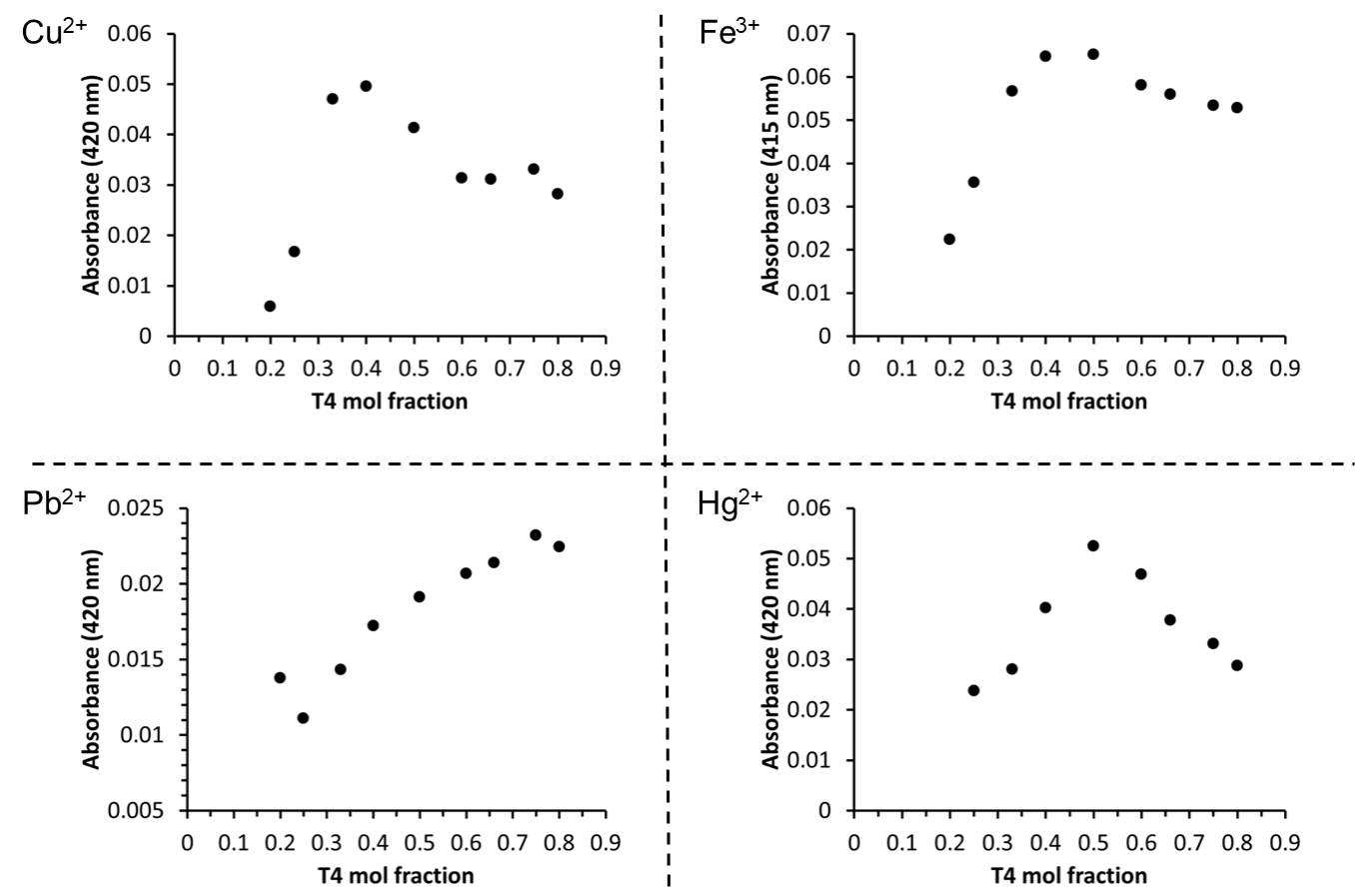

Figure 54: Job's plots for T4 with copper(II), iron(III), lead(II) and mercury(II) in $\mathrm{CH}_{3} \mathrm{CN}$ at a total concentration of $2 \times 10^{-5} \mathrm{M}$.

Though Job's plots were obtained for T4 with copper(II), iron(III), lead(II) and mercury(II), they are not consistent with the association constants determined through the fits to the absorption titration data (Figure 54). The best fit to the absorption titration data for copper(II), iron(III) and mercury(II) was obtained from using a model where these metals bind in a 2:1 stoichiometry (T4:metal) at low concentrations (

Table 12). Lead(II) is the only metal observed to bind at a 1:1 stoichiometry based on fits of the absorption titration data, consistent with the observed changes in the absorption and emission spectrum. Functionalization with the amino group results in an increased $K_{\mathrm{a}}$ of $\mathbf{T 4}\left(4.72 \times 10^{10} \mathrm{M}^{-2} \pm 7.42 \%\right)$ for mercury(II) as compared to the nonfunctionalized ligand (L2) $\left(5.37 \times 10^{9} \mathrm{M}^{-2} \pm 9.8 \%\right)$. 
Table 12: Association constants for T4 with copper(II), iron(III), lead(II) and mercury(II) in $\mathrm{CH}_{3} \mathrm{CN}$ obtained from absorption titrations.

\begin{tabular}{cc}
\hline Metal ion & $\boldsymbol{K}_{\mathbf{a}}$ \\
\hline Copper(II) & $5.23 \times 10^{10} \mathrm{M}^{-2}( \pm 8.98 \%)$ \\
\hline Iron(III) & $4.45 \times 10^{9} \mathrm{M}^{-2}( \pm 6.27 \%)$ \\
Lead(II) & $6.69 \times 10^{3} \mathrm{M}^{-1}( \pm 3.62 \%)$ \\
Mercury(II) & $4.72 \times 10^{10} \mathrm{M}^{-2}( \pm 7.42 \%)$ \\
\hline
\end{tabular}

The addition of copper(II), iron(III), lead(II) and mercury(II) to T4 results in similar absorption spectra, though with different $\lambda_{\max }$. Since the spectra are quite similar it is difficult to determine whether various coordination modes are in fact occurring at low concentrations of metal, thus ${ }^{1} \mathrm{H}$ NMR experiments were performed to identify what coordination events occur. Addition of lead(II) and iron(III) initially causes a downfield shift of the pyridyl peaks with broadening of the amino nitrogen peaks, suggesting N,S,N chelation occurs initially with these metals. Low concentrations of copper(II) and mercury(II) appear to result in a minimal shift of the pyridyl peaks with subsequent broadening and disappearance of the amino peaks. For both of these metals no significant downfield shift of the pyridyl peaks is observed until 1.00 equivalent of either copper(II) 
or mercury(II) had been added, suggesting chelation of the amino nitrogens occurs initially at low concentrations.
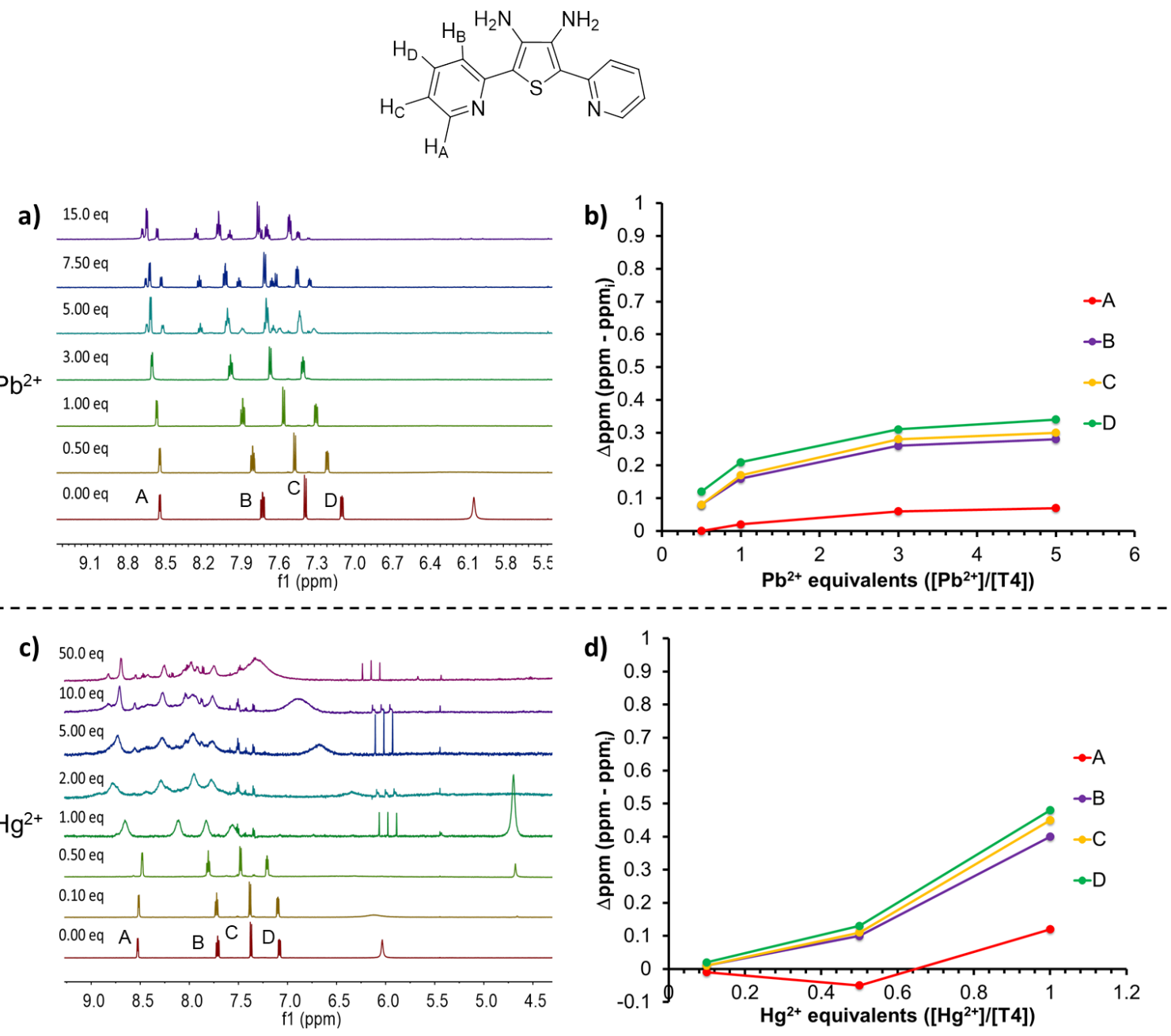

Figure 55: ${ }^{1} \mathrm{H}$ NMR titration of T4 at $4 \times 10^{-3} \mathrm{M}$ with increasing amounts of lead(II) and mercury(II) in $\mathrm{CD}_{3} \mathrm{CN}$. The magnitude of the chemical shift is plotted for each peak with increasing equivalents of (b) lead(II), and (d) mercury(II).

In the case of mercury(II) several distinctions are noted. At 0.50 equivalents of mercury(II) an apparent amino peak begins to arise at approximately $4.70 \mathrm{ppm}$, shifted upfield from the original chemical shift of the ligand. At 1.00 equivalent the intensity of 
this peak is at the highest and integrates to two protons relative to the pyridyl peaks, with concurrent broadening and downfield shifts of the pyridyl peaks. Several other peaks at lower intensities are also observed at this concentration suggesting multiple species are present in solution. The downfield shift and broadening of the pyridyl peaks suggests that when mercury(II) is at an equivalent concentration of $\mathbf{T 4}, \mathrm{N}, \mathrm{S}, \mathrm{N}$-chelation is the preferred coordination mode. Greater concentrations of mercury(II) then results in a downfield shift of this amino peak, suggesting possible formation of a bimetallic species in solution though this is unclear due to an increase in the number of peaks. 


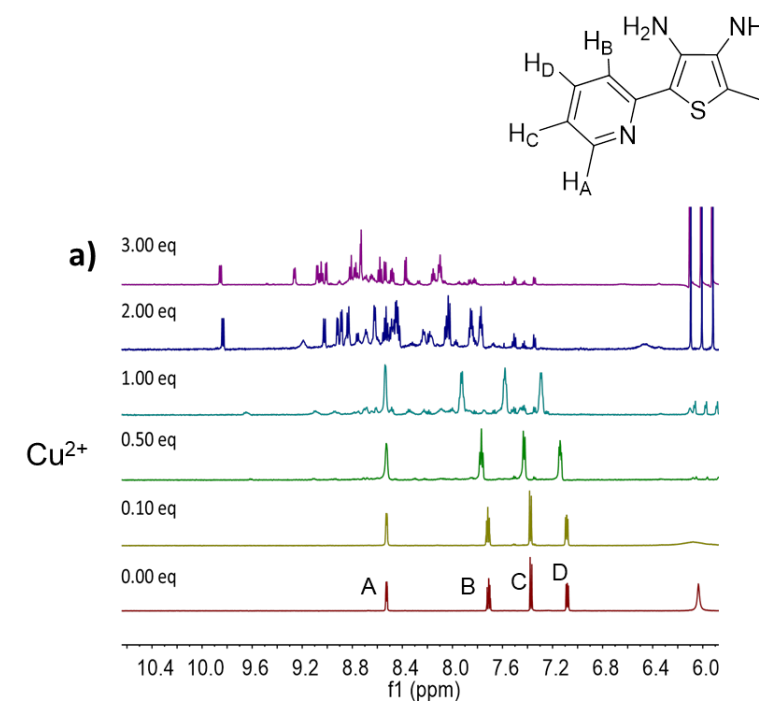<smiles>C1=CCC=C1</smiles>
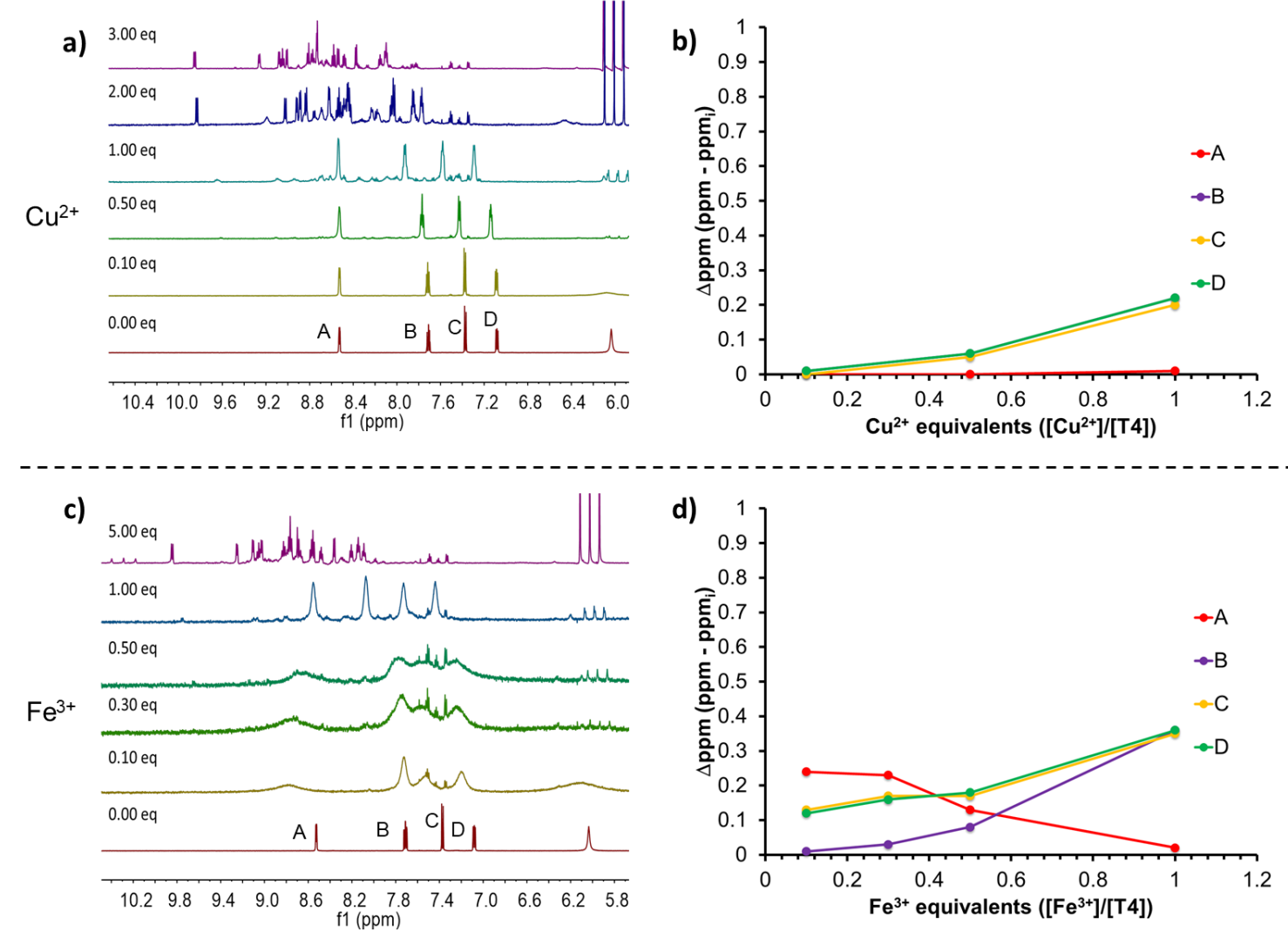

Figure 56: ${ }^{1} \mathrm{H}$ NMR titration of T4 at $4 \times 10^{-3} \mathrm{M}$ with increasing amounts of copper(II) and iron(III) in $\mathrm{CD}_{3} \mathrm{CN}$. The magnitude of the chemical shift is plotted for each peak with increasing equivalents of (b) copper(II), and (d) iron(III).

For all metals higher concentrations appear to result in the formation of multiple products in solution, as evidenced by an increase in the number of peaks in the NMR spectrum. Multiple species in solution is consistent with observations made during the emission titrations, which showed two distinct emissions occurring at two separate wavelengths. Similar to $\mathbf{T} 2$ an increase in the number of peaks suggests chelation of the pyridyl nitrogen to the heteroatom of the functional group, in this case the amino 
nitrogen. Chelation of the pyridyl and amino nitrogen atoms would result in an asymmetric complex with eight distinct proton environments. In the case of mercury(II) the broadening of the peaks makes it difficult to determine the exact number of peaks or integrations, thus it is difficult to determine the structures of the complexes. Similarly overlap of the peaks in the case of high concentrations of copper(II) and iron(III) make it difficult to interpret the spectra. High concentrations of lead(II) provide the sharpest peaks which allow for ease of interpretation. In this case a total of 12 peaks are observed, where eight of these protons are at lower intensity and have equivalent integrations. The remaining four protons have equivalent integrations, suggesting both $\mathrm{N}, \mathrm{S}, \mathrm{N}$-chelation as well as N,N-chelation at high concentrations of lead(II).

Previous work on mercury(II) sensors has suggested that amino nitrogens offer good selectivity for the mercury(II) ion, by an increased affinity. ${ }^{46,93-95}$ Since it appears from ${ }^{1} \mathrm{H}$ NMR experiments that initial coordination of $\mathbf{T 4}$ occurs via N,N-chelation, I therefore expected a high selectivity for mercury(II) with this sensor. Of the metals tested cobalt(II), iron(II), and zinc(II) result in an absorption response. Subsequent addition of mercury(II) appears to result in displacement of both cobalt(II) and zinc(II), and an increase in absorbance occurs after addition to the solution containing iron(II). Though T4 appears selective for mercury(II) against these metals, competitive binding is observed for copper(II), iron(III), manganese(II) and lead(II) thus the amino groups did not successfully enhance the selectivity for the mercury(II) ion. Of the metals tested T4 appears to be the most selective for iron(III), though some competitive binding appears to occur with copper(II). 

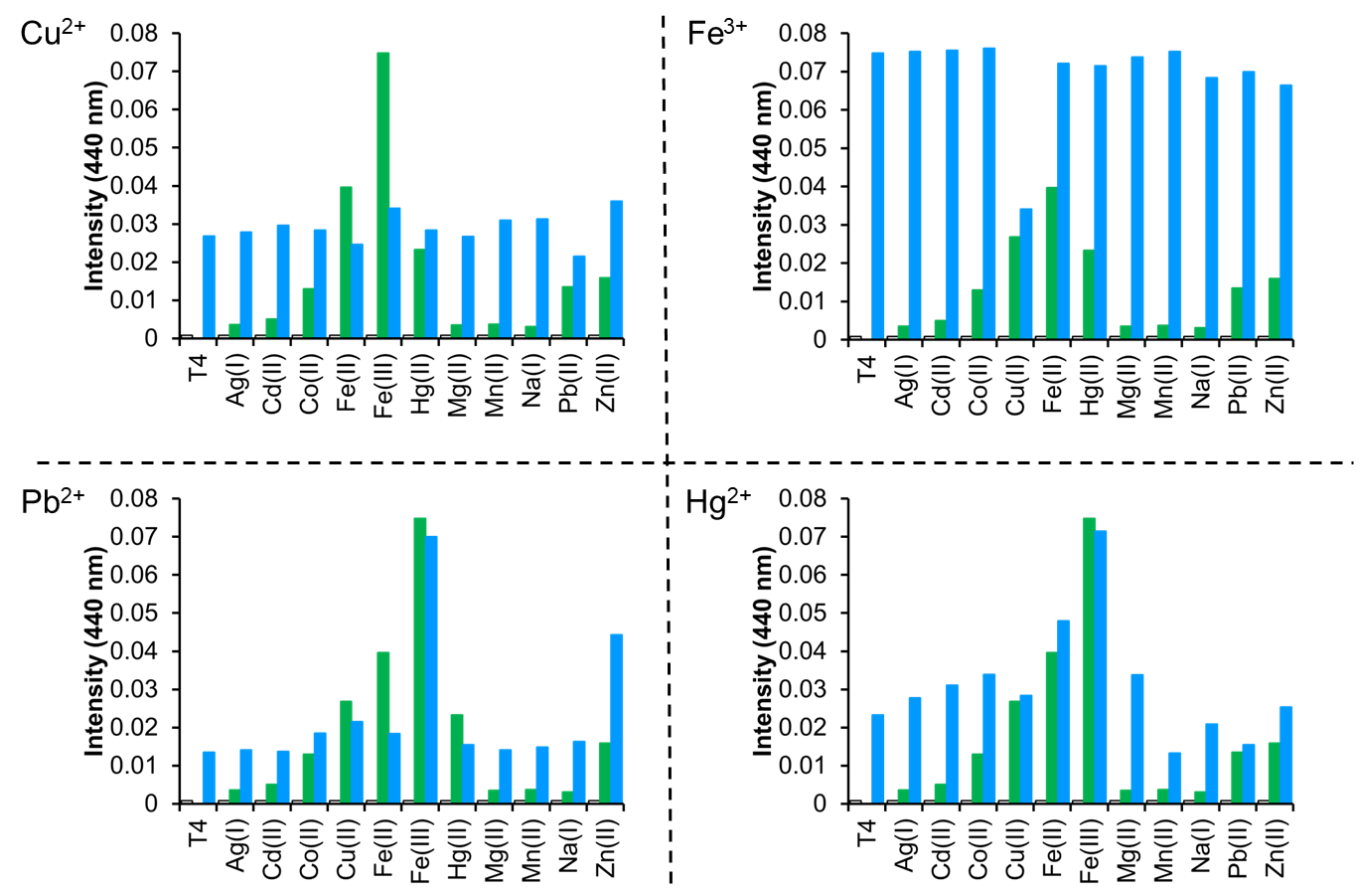

Figure 57: Absorption at $440 \mathrm{~nm}$ of T4 (white bars), after the addition of 5 eq. of metal ion (green bars), and subsequent addition of 5 eq. of either copper(II), iron(III), lead(II) or mercury(II) perchlorate (blue bars) in $\mathrm{CH}_{3} \mathrm{CN}$.

\subsection{Conclusion}

The primary goal of this project was to determine if functionalization of the thiophene offered a greater binding affinity for the thienyl sulfur, and thus greater selectivity for soft Lewis acids such as mercury(II). The photophysical responses of several thiophene-functionalized sensors to copper(II), iron(III), lead(II) and mercury(II) were measured. An improved selectivity for mercury(II) was observed in the case of T1 and T2, though iron(III) was still observed to bind competitively to these sensors. Functionalization did offer greater binding affinity ( $K_{\mathrm{a}}$ values) for mercury(II) in the case of T2-T4 though these values (T2-T4: $200 \mathrm{ppb)} \mathrm{were} \mathrm{still} \mathrm{far} \mathrm{from} \mathrm{meeting} \mathrm{EPA}$ standards (2 ppb). In addition to evaluating the change in selectivity and binding affinity, 
the photophysical response to other transition metals was observed to evaluate their efficacy as multiplex sensors. Though $\mathbf{T 1}$ and $\mathbf{T 3}$ provided a general response to these metals, T2 and T4 were both observed to have unique, specific responses to the metal added in solution. In the case of $\mathbf{T} 2$ the $\lambda_{\max }$ was well separated at low concentrations of metal suggesting this sensor could serve as a useful model for the development of a multiplex sensor. Interestingly $\mathbf{T 3}$ also seemed promising for the development of a multiplex sensor for the specific detection of copper(II) and iron(III) in biological systems due to the unique absorption response to copper(II) observed in the presence of sodium(I). The availability of several coordination modes are believed to facilitate this multiplex detection, as well as the increased number of products that were observed for T2 and $\mathbf{T} 4$ compared to the 2 observed for $\mathbf{L} 2$. 


\section{Chapter 7. Concluding Remarks}

The work described in this thesis has focused on several sets of thiophene-based molecular sensors. These projects began from the first generation of sensors that were developed, described in Chapter 3. Following Chapter 3 we have discussed different modifications that have been made to the first generation of sensors in order to improve

the overall $K_{\mathrm{a}}$ for mercury(II). While each modification was generally successful in improving the overall association constant for mercury(II), there were consistently issues with selectivity as iron(III) was shown to be a competitive binder in all cases. Though selectivity remains an issue to be addressed we have shown the efficacy of implementing thiophene in a molecular sensor system for soft Lewis acids such as mercury(II). Furthermore this work has revealed that functionalization of the thiophene ring with electron donating groups maintains useful photophysical properties, while also improving the overall $K_{\mathrm{a}}$. This is in direct contrast to using dibenzothiophene which improved the $K_{\mathrm{a}}$ but had less than ideal photophysical properties. Additionally, promising models for the production of a multiplex detector and ratiometric lead(II) sensor have been developed.

Initial work began with three small-molecule thiophene-based sensors, described in Chapter 3. The goal of this project was to determine the efficacy of thiophene in a molecular sensor system. To promote coordination to the thienyl sulfur pyridyl chelating groups were employed with good success. There were a total of three sensors in this project; a bidentate ligand bearing a single thiophene and pyridine moiety (L1), and two tridentate ligands. The two tridentate ligands had a central thiophene ring with two 
flanking pyridine rings (L2), and a central pyridine ring with two thiophene chelators (L3). These sensors offered a ratiometric fluorescent and absorbance response and good selectivity against other transition metal sensors, except for iron(III). Furthermore L2 was observed to form two products dependent on the amount of mercury(II) in solution. The primary drawback to these sensors was the generally low $K_{\mathrm{a}}$ values obtained which limited their applicability as molecular sensors. Following this, a series of sensors with a benzothiophene ring in replacement of the thiophene ring of $\mathbf{L} \mathbf{1}$ and the selectivity of various chelating groups was investigated.

The goal of the work described in Chapter 4 was two-fold. First we aimed to assess the efficacy of implementing dibenzothiophene, rather than thiophene, as this ligand has been shown to have a greater overall $K_{\mathrm{a}}$ for metal ions. Second we varied the pyridyl chelating groups used in Chapter 3 to determine whether different nitrogen or sulfur donors would provide better selectivity and $K_{\mathrm{a}}$ values. To determine this, the thiophene ring of $\mathbf{L 1}$ for dibenzothiophene was substituted to develop BT1. Several other bidentate ligands were also synthesized containing imidazole (BT2), and thiazole (BT3). Though these sensors were shown to have a greater overall $K_{\mathrm{a}}$, less than ideal photophysics were obtained, as the absorptivities and quantum yields were quite low. Furthermore, the photophysical response of these sensors would eventually red-shift towards the visible region however this would not afford a colored solution with the addition of metal. The pyridyl chelating group was determined to be the most ideal chelator as it afforded the best $K_{\mathrm{a}}$ and was the most selective for mercury(II) against most transition metal ions. 
Following this we chose to focus my efforts on modification of $\mathbf{L} \mathbf{2}$, using electron donating groups to increase the $K_{\mathrm{a}}$ instead of dibenzothiophene.

The ability of $\mathbf{L} \mathbf{2}$ to form two products in solution would allow for a greater detectable range of concentrations for sensing applications, prompting my modification of this sensor. Furthermore this ligand was shown to have the greatest overall $K_{\mathrm{a}}$ from the first generation of sensors, as well as good selectivity for mercury(II). To improve this design the pyridyl rings were functionalized with alcohol (P1), octaethyleneglycol monomethyl ether (P2) and amine (P3) groups. Functionalization of these electron donating groups was done to improve the overall $K_{\mathrm{a}}$, as well as improve the water-solubility of the sensors. As described in Chapter 5 these sensors were shown to be water-soluble however they had a less than ideal photophysical response in water. The ideal photophysical properties of $\mathbf{L 2}$ were preserved in organic solvents upon functionalization, though the quantum yields of P1-P3 did decrease relative to $\mathbf{L 2}$. Furthermore the photophysical response resulted again in a ratiometric red-shift of the absorption and emission spectra of the sensors ideal for sensing applications. The $K_{\mathrm{a}}$ values were also shown to improve over the nonfunctionalized ligand (L2), however this translated to an overall increased affinity for other metal ions and thus a loss in selectivity for mercury(II).

The final project, described in Chapter 6, involved functionalization of the thiophene ring in order to improve the thienyl sulfur binding affinity to improve the overall $K_{\mathrm{a}}$ for soft Lewis acids such as mercury(II). This was done with methoxy (T1), alcohol (T2), octaethyleneglycol monomethyl ether (T3) and amine (T4) groups. Though 
the overall $K_{\mathrm{a}}$ for mercury(II) was improved over previous generations of ligands, these sensors were still not selective for the mercury(II) ion. However functionalization at this position facilitated formation of several products in solution, further expanding the range of detection for a given metal analyte. Furthermore $\mathbf{T} 2$ and $\mathbf{T 3}$ showed specific responses to several metal ions suggesting they could be used as a model for the development of small-molecule multiplex detectors. The $K_{\mathrm{a}}$ values for other metals were also quite high and in fact $\mathbf{T 3}$ is capable of detecting as little as $0.13 \mathrm{ppm}$ of copper(II), meeting EPA standards (2 ppm). In addition to this both $\mathbf{T 1}$ and $\mathbf{T 3}$ were shown to have ratiometric fluorescent response to lead(II), suggesting they could be used as a model for the development as a ratiometric sensor for this metal.

In conclusion we have implemented several strategies to improve the overall $K_{\mathrm{a}}$ for several thiophene-based small molecule sensors. While a selective response to mercury(II) was not achieved, several interesting properties of these ligands was revealed upon modification of the sensors. Perhaps most interesting was the ability of $\mathbf{L} \mathbf{2}$ to form multiple products in solution, which could be exploited in sensing applications to detect a larger range of concentrations of a given metal analyte. This was shown to be expanded by functionalization of the thiophene ring, which allowed for additional coordination modes and thus increased the number of spectroscopically distinct products observed in solution in the case of $\mathbf{T} 2$ and $\mathbf{T} 4$. Furthermore this simple modification allowed $\mathbf{T} 2$ to provide a specific response to up to four different metals in solution. This could potentially be applied towards the development of a small-molecule multiplex detector. With the conclusion of this work we hope to have shown the efficacy of implementing 
thiophene in molecular sensor systems for the development of a selective sensor for soft Lewis acids such as mercury(II). 


\section{REFERENCES}

(1) Tchounwou, P. B., Yedjou, C. G., Patlolla, A. K., and Sutton, D. J. (2012) Heavy Metals Toxicity and the Environment. EXS 101, 1-30.

(2) Hernberg, S. (2000) Lead poisoning in a historical perspective. Am. J. Ind. Med. 38, $244-254$.

(3) Arruti, A., Fernández-Olmo, I., and Irabien, Á. (2010) Evaluation of the contribution of local sources to trace metals levels in urban PM2.5 and PM10 in the Cantabria region (Northern Spain). J. Environ. Monit. 12, 1451.

(4) Ekino, S., Susa, M., Ninomiya, T., Imamura, K., and Kitamura, T. (2007) Minamata disease revisited: An update on the acute and chronic manifestations of methyl mercury poisoning. J. Neurol. Sci. 262, 131-144.

(5) Jaishankar, M., Tseten, T., Anbalagan, N., Mathew, B. B., and Beeregowda, K. N. (2014) Toxicity, mechanism and health effects of some heavy metals. Interdiscip. Toxicol. 7, 60-72.

(6) Valeur, B., and Leray, I. (2000) Design principles of fluorescent molecular sensors for cation recognition. Coord. Chem. Rev. 205, 3-40.

(7) Saleem, M., and Lee, K. H. (2015) Optical sensor: a promising strategy for environmental and biomedical monitoring of ionic species. $R S C A d v .5,72150-72287$. 
(8) Nolan, E. M., and Lippard, S. J. (2008) Tools and tactics for the optical detection of mercuric ion. Chem. Rev. 108, 3443-3480.

(9) Kim, H. N., Ren, W. X., Kim, J. S., and Yoon, J. (2012) Fluorescent and colorimetric sensors for detection of lead, cadmium, and mercury ions. Chem. Soc. Rev. 41, 3210.

(10) US EPA. (1994) Determination of Mercury in Water By Cold Vapor Atomic Absorption Spectrometry Method 245.1. Environ. Monit. Syst. Lab. Off. Res. Dev.

(11) Sahoo, S. K., Sharma, D., Bera, R. K., Crisponi, G., and Callan, J. F. (2012) Iron(III) selective molecular and supramolecular fluorescent probes. Chem. Soc. Rev. 41, 7195.

(12) Halliwell, B., and Gutteridge, J. M. C. (1990) Role of free radicals and catalytic metal ions in human disease: An overview. Methods Enzymol. 186, 1-85.

(13) Valko, M., Morris, H., and Cronin, M. (2005) Metals, Toxicity and Oxidative Stress. Curr. Med. Chem. 12, 1161-1208.

(14) Giampietro, R., Spinelli, F., Contino, M., and Colabufo, N. A. (2018) The Pivotal Role of Copper in Neurodegeneration: A New Strategy for the Therapy of Neurodegenerative Disorders. Mol. Pharm. 15, 808-820.

(15) Gaggelli, E., Kozlowski, H., Valensin, D., and Valensin, G. (2006) Copper homeostasis and neurodegenerative disorders (Alzheimer's, prion, and Parkinson's diseases and amyotrophic lateral sclerosis). Chem. Rev. 106, 1995-2044.

(16) Lovell, M. ., Robertson, J. ., Teesdale, W. ., Campbell, J. ., and Markesbery, W. . 
(1998) Copper, iron and zinc in Alzheimer's disease senile plaques. J. Neurol. Sci. 158, $47-52$.

(17) Cheignon, C., Tomas, M., Bonnefont-Rousselot, D., Faller, P., Hureau, C., and Collin, F. (2018) Oxidative stress and the amyloid beta peptide in Alzheimer's disease. Redox Biol. 14, 450-464.

(18) EPA Office of Water: Washington DC. (2010) National Primary Drinking Water Regulations for Lead and Copper. Fed. Regist.

(19) Hynes, M. J., and Jonson, B. (1997) Lead, glass and the environment. Chem. Soc. Rev. 26, 133.

(20) EPA Office of Water: Washington DC. (2001) Mercury Update: Impact on Fish Advisories Mercury. EPA Fact Sheet EPA- 823-F-01-011.

(21) Hancock, R. D. (1992) Chelate ring size and metal ion selection. The basis of selectivity for metal ions in open-chain ligands and macrocycles. J. Chem. Educ. 69, 615.

(22) Cockrell, G. M., Zhang, G., VanDerveer, D. G., Thummel, R. P., and Hancock, R. D. (2008) Enhanced metal ion selectivity of 2,9-di-(pyrid-2-yl)-1,10-phenanthroline and its use as a fluorescent sensor for cadmium(II). J. Am. Chem. Soc. 130, 1420-1430.

(23) Kim, H. N., Lee, M. H., Kim, H. J., Kim, J. S., and Yoon, J. (2008) A new trend in rhodamine-based chemosensors: application of spirolactam ring-opening to sensing ions. Chem. Soc. Rev. 37, 1465-72. 
(24) Leray, I., and Valeur, B. (2009) Calixarene-based fluorescent molecular sensors for toxic metals. Eur. J. Inorg. Chem. 3525-3535.

(25) Carter, K. P., Young, A. M., and Palmer, A. E. (2014) Fluorescent sensors for measuring metal ions in living systems. Chem. Rev. 114, 4564-601.

(26) Tsien, R., and Poenie, M. (1986) Fluorescence ratio imaging: a new window into intracellular ionic signaling. Trends Biochem. Sci. 11, 450-455.

(27) Chen, X., Pradhan, T., Wang, F., Kim, J. S., and Yoon, J. (2012) Fluorescent chemosensors based on spiroring-opening of xanthenes and related derivatives. Chem. Rev. 112, 1910-1956.

(28) Xu, N. Z., Liu, M. M., Ye, M. A., Yao, Y. W., Zhou, Y., Wu, G. Z., and Yao, C. (2017) A Rhodamine-naphthalimide conjugated chemosensor for ratiometric detection $\mathrm{Hg}^{2+}$ in actual aqueous samples. J. Lumin. 188, 135-140.

(29) Chen, S., Wang, W., Yanf, M., Tu, Q., Chen, S.-W., Li, T., Yuan, M.-S., and Wang, J. (2018) 2-Hydroxy benzothiazole modified rhodol: aggregation-induced emission and dual-channel fluorescence sensing of $\mathrm{Hg}^{2+}$ and $\mathrm{Ag}^{+}$ions. Sensors Actuators B Chem. 255, 2086-2094.

(30) Su, W., Yuan, S., and Wang, E. (2017) A Rhodamine-Based Fluorescent Chemosensor for the Detection of $\mathrm{Pb}^{2+}, \mathrm{Hg}^{2+}$ and $\mathrm{Cd}^{2+}$. J. Fluoresc. 27, 1871-1875.

(31) Jo, T. G., Jung, J. M., Han, J., Lim, M. H., and Kim, C. (2017) A single fluorescent 
chemosensor for multiple targets of $\mathrm{Cu}^{2+}, \mathrm{Fe}^{2+/ 3+}$ and $\mathrm{Al}^{3+}$ in living cells and a nearperfect aqueous solution. $R S C A d v .7,28723-28732$.

(32) Saleem, M., Kang, S. K., and Lee, K. H. (2015) Microwave assisted synthesis of a novel optical chemosensor for selective Fe3+detection. J. Lumin. 162, 14-24.

(33) Chhatwal, M., Kumar, A., Singh, V., Gupta, R. D., and Awasthi, S. K. (2015) Addressing of multiple-metal ions on a single platform. Coord. Chem. Rev. 292, 30-55.

(34) Aydin, M., Carter-conger, J., Gao, N., Gilmore, D. F., Ricke, S. C., and Ahn, S. (2018) Molecular identification of common Salmonella serovars using multiplex DNA sensor-based suspension array.

(35) Huang, H., Chen, S., Liu, F., Zhao, Q., Liao, B., Yi, S., and Zeng, Y. (2013) Multiplex plasmonic sensor for detection of different metal ions based on a single type of gold nanorod. Anal. Chem. 85, 2312-2319.

(36) Zhang, Y., Zuo, P., and Ye, B. C. (2015) A low-cost and simple paper-based microfluidic device for simultaneous multiplex determination of different types of chemical contaminants in food. Biosens. Bioelectron. 68, 14-19.

(37) Freeman, R., Finder, T., and Willner, I. (2009) Multiplexed analysis of $\mathrm{Hg}^{2+}$ and $\mathrm{Ag}^{+}$ ions by nucleic acid functionalized $\mathrm{CdSe} / \mathrm{ZnS}$ quantum dots and their use for logic gate operations. Angew. Chemie - Int. Ed. 48, 7818-7821.

(38) Suzuki, M., Husimi, Y., Komatsu, H., Suzuki, K., and Douglas, a. K. T. (2008) 
Quantum Dot FRET Biosensors that Respond to $\mathrm{pH}$, to IrProteolytic or Nucleolytic Cleavage, to DNA Synthesis, or to a \rMultiplexing Combination. J. Am. Chem. Soc. 130, $5720-5725$.

(39) Prabpal, J., Vilaivan, T., and Praneenararat, T. (2017) Paper-Based Heavy Metal Sensors from the Concise Synthesis of an Anionic Porphyrin: A Practical Application of Organic Synthesis to Environmental Chemistry. J. Chem. Educ. 94, 1137-1142.

(40) Prével, C., Pellerano, M., Van, T. N. N., and Morris, M. C. (2014) Fluorescent biosensors for high throughput screening of protein kinase inhibitors. Biotechnol. J. 9, $253-265$.

(41) Valeur, B. (2001) Molecular Fluorescence Principles and Applications. Methods.

(42) Chen, G., Guo, Z., Zeng, G., and Tang, L. (2015) Fluorescent and colorimetric sensors for environmental mercury detection. Analyst 140, 5400-5443.

(43) Zhu, H., Fan, J., Wang, B., and Peng, X. (2015) Fluorescent, MRI, and colorimetric chemical sensors for the first-row d-block metal ions. Chem. Soc. Rev. 44, 4337-4366.

(44) Barbarella, G., Melucci, M., and Sotgiu, G. (2005) The versatile thiophene: An overview of recent research on thiophene-based materials. Adv. Mater. 17, 1581-1593.

(45) Kim, T. H., and Swager, T. M. (2003) A Fluorescent Self-Amplifying WavelengthResponsive Sensory Polymer for Fluoride Ions. Angew. Chemie - Int. Ed. 42, 4803-4806.

(46) Nolan, E. M., Ryu, J. W., Jaworski, J., Feazell, R. P., Sheng, M., and Lippard, S. J. 
(2006) Zinspy sensors with enhanced dynamic range for imaging neuronal cell zinc uptake and mobilization. J. Am. Chem. Soc. 128, 15517-15528.

(47) Tang, Y., He, F., Yu, M., Feng, F., An, L., Sun, H., Wang, S., Li, Y., and Zhu, D. (2006) A reversible and highly selective fluorescent sensor for mercury(II) using poly(thiophene)s that contain thymine moietiesa. Macromol. Rapid Commun. 27, 389392.

(48) Huang, W., Zhu, X., Wua, D., He, C., Hu, X., and Duan, C. (2009) Structural modification of rhodamine-based sensors toward highly selective mercury detection in mixed organic/aqueous media. Dalton Trans. 2, 10457-10465.

(49) Ding, J., Li, H., Wang, C., Yang, J., Xie, Y., Peng, Q., Li, Q., and Li, Z. (2015) “Turn-On” Fluorescent Probe for Mercury(II): High Selectivity and Sensitivity and New Design Approach by the Adjustment of the $\pi$-Bridge. ACS Appl. Mater. Interfaces 7, 11369-11376.

(50) Aydin, Z., Wei, Y., and Guo, M. (2014) An "off-on" optical sensor for mercury ion detection in aqueous solution and living cells. Inorg. Chem. Commun. 50, 84-87.

(51) Udhayakumari, D., Suganya, S., Velmathi, S., and MubarakAli, D. (2014) Naked eye sensing of toxic metal ions in aqueous medium using thiophene-based ligands and its application in living cells. J. Mol. Recognit. 27, 151-159.

(52) Perepichka, I. F., Perepichka, D. F., Meng, H., and Wudl, F. (2005) Light-emitting polythiophenes. Adv. Mater. 17, 2281-2305. 
(53) Yang, H., Zhou, Z., Huang, K., Yu, M., Li, F., Yi, T., and Huang, C. (2007) Multisignaling optical-electrochemical sensor for $\mathrm{Hg}^{2+}$ based on a rhodamine derivative with a ferrocene unit. Org. Lett. 9, 4729-4732.

(54) Angelici, R. J. (2001) Thiophenes in organotransition metal chemistry: Patterns of reactivity. Organometallics.

(55) du Toit, A., Landman, M., and Lotz, S. (1997) Synthesis and structure of bimetallic complexes with $\sigma, \pi$-bridging thienyl and benzothienyl ligands. J. Chem. Soc. Dalt. Trans. $2955-2962$.

(56) Thomas, S. W., Venkatesan, K., Müller, P., and Swager, T. M. (2006) Dark-field oxidative addition-based chemosensing: New bis-cyclometalated Pt(II) complexes and phosphorescent detection of cyanogen halides. J. Am. Chem. Soc. 128, 16641-16648.

(57) Li, X. H., Shi, Z., Wang, L., Cheng, X., Li, C., and Zhang, A. (2013) Chromogenic mercury ions recognition of a new ruthenium(II) complex with cyclometalated 2-(2thienyl)pyridine in $\mathrm{CH} 3 \mathrm{CN}$-aqueous system. Inorg. Chem. Commun. 29, 175-178.

(58) Gioia, G., Cecchi, P., Giordano, F., and Santini, C. (1996) Metal polypyrazolylborates. X. Thienylmercury (II) derivatives: the X-ray crystal structure of [(5-Me)Thien-2-yl]Hg-( $\pi-\mathrm{Pz}) 2 \mathrm{~B}(\mathrm{Pz})$ 2. J. Organomet. Chem. 515, $213-220$.

(59) Robertson, M. J., White, C. J., and Angelici, R. J. (1994) Sulfur-Coordinated Thiophene and Benzothiophene in $\mathrm{Cp}(\mathrm{NO})\left(\mathrm{PPh}_{3}\right) \mathrm{Re}(\text { thiophene) })^{+}$: Conversion to Thienyl and Thienylcarbene Complexes. J. Am. Chem. Soc. 116, 5190-5195. 
(60) Bell, N. A., Crouch, D. J., and Jaffer, N. E. (2004) Coordination complexes of 2thienyl- and 2-furyl-mercurials. Appl. Organomet. Chem. 18, 135-138.

(61) Dullaghan, C. A., Carpenter, G. B., Sweigart, D. A., Choi, D. S., Lee, S. S., and Chung, Y. K. (1997) Models for the Homogeneous Hydrodesulfurization of Thiophenes : Manganese-Mediated Carbon - Sulfur Bond Cleavage and Hydrogenation Reactions. Organometallics 7333, 5688-5695.

(62) Bianchini, C., Gatteschi, D., Giambastiani, G., Rios, I. G., Ienco, A., Laschi, F., Mealli, C., Meli, A., Sorace, L., Toti, A., and Vizza, F. (2007) Electronic influence of the thienyl sulfur atom on the oligomerization of ethylene by cobalt(II) 6-(thienyl)-2(imino)pyridine catalysis. Organometallics 26, 726-739.

(63) Schallenberg, D., Neubauer, A., Erdmann, E., Tänzler, M., Villinger, A., Lochbrunner, S., and Seidel, W. W. (2014) Dinuclear Ru/Ni, Ir/Ni, and Ir/Pt Complexes with Bridging Phenanthroline-5,6-dithiolate: Synthesis, Structure, and Electrochemical and Photophysical Behavior. Inorg. Chem. 53, 8859-8873.

(64) Yamashita, M., and Hartwig, J. F. (2004) Synthesis, Structure, and Reductive Elimination Chemistry of Three-Coordinate Arylpalladium Amido Complexes. J. Am. Chem. Soc. 126, 5344-5345.

(65) Kozhevnikov, D. N., Kozhevnikov, V. N., Shafikov, M. Z., Prokhorov, A. M., Bruce, D. W., and Williams, J. a G. (2011) Phosphorescence vs fluorescence in cyclometalated platinum(II) and Iridium(III) complexes of (Oligo)thienylpyridines. 
Inorg. Chem. 50, 3804-3815.

(66) Deeming, A. J., Jayasuriya, S. N., Arce, A. J., and De Sanctis, Y. (1996) Incorporation of Thiophene Rings into Tri- and Tetraruthenium Clusters via Cyclometalation and $\mathrm{C}-\mathrm{P}$ Bond Cleavage of the Ligand Diphenyl-2-thienylphosphine. Organometallics 15, 786-793.

(67) Oilunkaniemi, R., Laitinen, R. S., and Ahlgrén, M. (1999) The NMR spectroscopic and X-ray crystallographic study of the oxidative addition of bis(2-thienyl) diselenide to zerovalent palladium and platinum centers. J. Organomet. Chem. 587, 200-206.

(68) Benson, J. W., and Angelici, R. J. (1992) Equilibrium Studies of the Displacement of $\eta^{1}(\mathrm{~S})$-Thiophenes $(\mathrm{Th})$ from $\mathrm{Cp}(\mathrm{CO})(\mathrm{PPh},) \mathrm{Ru}\left(\eta^{1}(\mathrm{~S})-\mathrm{Th}\right)^{+}$. Organometallics 922-927.

(69) Alvarez, M., Lugan, N., and Mathieu, R. (1993) Synthesis and Evaluation of the Bonding Properties of 2,5-Bis(2-(diphenylphosphino)ethyl)thiophene: A New Potentially Tridentate Ligand. Inorg. Chem. 32, 5652-5657.

(70) Choi, M., and Angelici, R. J. (1991) Sulfur-Coordinated Thiophene and Dibenzothiophene in Cp'( CO), Re(thiophene) Complexes 2442, 2436-2442.

(71) Choi, M.-G., and Angelici, R. J. (1989) Reaction of the sulfur-coordinated thiophene in $\mathrm{Cp}^{*}(\mathrm{CO})_{2} \mathrm{Re}\left(\mathrm{SC}_{4} \mathrm{H}_{4}\right)$ to give the Thiophene-Bridged $\mathrm{Cp}^{*}(\mathrm{Co})_{2} \mathrm{Re}\left(\mu-\mathrm{SC}_{4} \mathrm{H}_{4}\right) \mathrm{Fe}(\mathrm{CO})_{3}$. J. Am. Chem. Soc. 58, 8753-8754.

(72) Benson, J. W., and Angelici, R. J. (1993) Equilibrium and kinetic studies of sulfur- 
coordinated thiophenes (th) in $\mathrm{Cp}(\mathrm{CO}) 2 \mathrm{Ru}\left(\eta^{1}(\mathrm{~S}) \text {-th }\right)^{+}$and $\mathrm{Cp}(\mathrm{CO})\left(\mathrm{PPh}_{3}\right) \mathrm{Ru}\left(\eta^{1}(\mathrm{~S}) \text {-th }\right)^{+}$: models for thiophene adsorption on hydrodesulfurization catalysts. Organometallics 12, $680-687$.

(73) Garcia, J. J., Mann, B. E., Adams, H., Bailey, N. a., and Maitlis, P. M. (1995) Equilibria of the Thiametallacycles with Tris(triethylphosphine)platinum(0) and Dibenzothiophene, Benzothiophene, or Thiophene: The Hydrodesulfurization Reaction. J. Am. Chem. Soc. 117, 2179-2186.

(74) Reynolds, M. A., Guzei, I. A., Logsdon, B. C., Thomas, L. M., Jacobson, R. A., and Angelici, R. J. (1999) Transition Metal Complexes of Chromium , Molybdenum, Tungsten, and Manganese Containing $\eta^{1}(\mathrm{~S})-2,5$-Dimethylthiophene, Benzothiophene, and Dibenzothiophene Ligands. Organometallics 1, 4075-4081.

(75) Dorta, R., Konstantinovski, L., Shimon, L. J. W., Ben-David, Y., and Milstein, D. (2003) Chelation versus cyclometalation in a cationic Dppn-RhI complex - a unique rearrangement of norbornadiene via $\mathrm{C}-\mathrm{H}$ activation of the pyridazine ring. Eur. J. Inorg. Chem. 70-76.

(76) Yigit, D., Aykan, M., and Gullu, M. (2018) Substituent Effect on Supercapacitive Performances of Conducting. J. Polym. Sci. Part A Polym. Chem. 56, 480-495.

(77) Traina, C. A., Bakus, R. C., and Bazan, G. C. (2011) Design and synthesis of monofunctionalized, water-soluble conjugated polymers for biosensing and imaging applications. J. Am. Chem. Soc. 133, 12600-12607. 
(78) Dziedzic, P., Cisneros, J. A., Robertson, M. J., Hare, A. A., Danford, N. E., Baxter, R. H. G., and Jorgensen, W. L. (2015) Design, synthesis, and protein crystallography of biaryltriazoles as potent tautomerase inhibitors of macrophage migration inhibitory factor. J. Am. Chem. Soc. 137, 2996-3003.

(79) Thordarson, P. (2011) Determining association constants from titration experiments in supramolecular chemistry. Chem. Soc. Rev. 40, 1305-1323.

(80) M. J. Frisch, G. W. Trucks, H. B. Schlegel, G. E. Scuseria, M. A. Robb, J. R. Cheeseman, G. Scalmani, V. Barone, B. Mennucci, G. A. Petersson, H. Nakatsuji, M. Caricato, X. Li, H. P. Hratchian, A. F. Izmaylov, J. Bloino, G. Zheng, J. L. Sonnenberg, M. Had, and D. J. F. (2009) Gaussian 09, Revision D.01. Gaussian, Inc., Wallingford CT.

(81) Miehlich, B., Savin, A., Stoll, H., and Preuss, H. (1989) Results obtained with the correlation energy density functionals of becke and Lee, Yang and Parr. Chem. Phys. Lett. 157, 200-206.

(82) Lee, C., Yang, W., and Parr, R. G. (1988) Development of the Colle-Salvetti correlation-energy formula into a functional of the electron density. Phys. Rev. B 37, $785-789$.

(83) Shigemoto, A. K., Virca, C. N., Underwood, S. J., Shetterly, L. R., and Mccormick, T. M. (2016) Thiophene-based fluorescent mercury-sensors. J. Coord. Chem. 8972, 1-9.

(84) Meth-Cohn, O., and Jiang, H. (1998) Ligands containing alternating 2,6-linked 
pyridine and 2,5-linked thiophene units 1. J. Chem. Soc. Perkin Trans. 1 3737-3746.

(85) Thathong, Y., Jitchati, R., and Wongkhan, K. (2012) Air-Stable AnthracenePhosphine Oxide Adduct Ligand in Pd Catalysed Suzuki-Miyaura Reactions. APCBEE Procedia 3, 154-160.

(86) Orellana, G., Alvarez Ibarra, C., and Santoro, J. (1988) Hydrogen-1 and carbon-13 NMR coordination-induced shifts in a series of tris( $\hat{I} \pm$-diimine)ruthenium(II) complexes containing pyridine, pyrazine, and thiazole moieties. Inorg. Chem. 27, 1025-1030.

(87) Shibue, M., Hirotsu, M., Nishioka, T., and Kinoshita, I. (2008) Ruthenium and Rhodium Complexes with Thiolate-Containing Pincer Ligands Produced by C-S Bond Cleavage of Pyridyl-Substituted Dibenzothiophenes Ruthenium and Rhodium Complexes with Thiolate-Containing Pincer Ligands Produced by C - S Bond Cleavage of Pyr 44754483.

(88) Dziedzic, P., Cisneros, J. A. J. A., Robertson, M. J., Hare, A. A., Danford, N. E., Baxter, R. H. G., Jorgensen, W. L., Nadia, E., Baxter, R. H. G., and Jorgensen, W. L. (2015) Design , Synthesis, and Protein Crystallography of Biaryltriazoles as Potent Tautomerase Inhibitors of Macrophage Migration Inhibitory Factor. J. Am. Chem. Soc. 137, 2996-3003.

(89) Duarte, K., Justino, C. I. L., Freitas, A. C., Gomes, A. M. P., Duarte, A. C., and Rocha-Santos, T. A. P. (2015) Disposable sensors for environmental monitoring of lead, cadmium and mercury. TrAC Trends Anal. Chem. 64, 183-190. 
(90) Yoon, J., Ohler, N. E., Vance, D. H., Aumiller, W. D., and Czarnik, A. W. (1997) A Fluorescent Chemosensor Signalling Only $\mathrm{Hg}(\mathrm{II})$ and $\mathrm{Cu}(\mathrm{II})$ in Water. Tetrahedron Lett. $38,3845-3848$.

(91) Pignataro, L., Benaglia, M., Annunziata, R., Cinquini, M., and Cozzi, F. (2006) Structurally simple pyridine $\mathrm{N}$-oxides as efficient organocatalysts for the enantioselective allylation of aromatic aldehydes. J. Org. Chem. 71, 1458-1463.

(92) Barlow, S., Zhang, Q., Kaafarani, B. R., Risko, C., Amy, F., Chan, C. K., Domercq, B., Starikova, Z. A., Antipin, M. Y., Timofeeva, T. V., Kippelen, B., Brédas, J. L., Kahn, A., and Marder, S. R. (2007) Synthesis, ionisation potentials and electron affinities of hexaazatrinaphthylene derivatives. Chem. - A Eur. J. 13, 3537-3547.

(93) Zhu, X., and Alexandratos, S. D. (2005) Polystyrene-supported amines: Affinity for mercury (II) as a function of the pendant groups and the $\mathrm{Hg}$ (II) counterion. Ind. Eng. Chem. Res. 44, 8605-8610.

(94) Atia, A. A., Donia, A. M., and Elwakeel, K. Z. (2005) Selective separation of mercury (II) using a synthetic resin containing amine and mercaptan as chelating groups. React. Funct. Polym. 65, 267-275.

(95) Li, Q., Zhou, X., and Xing, D. (2010) Rapid and highly sensitive detection of mercury ion $\left(\mathrm{Hg}^{2+}\right)$ by magnetic beads-based electrochemiluminescence assay. Biosens. Bioelectron. 26, 859-862. 